\title{
Organocatalytic Enantioselective Construction of Heterocycle-Substituted Styrenes with Chiral Atropisomerism
}

Quan-Zhe Li, Peng-Fei Lian, Fu-Xin Tan, Guo-Dong Zhu*, Chao Chen, Yu Hao, Wei Jiang, Xun-Hui Wang, Jia Zhou, and Shu-Yu Zhang*

Shanghai Key Laboratory for Molecular Engineer of Chiral Drugs, School of Chemistry and Chemical Engineering \& Frontiers Science Center for Transformative Molecules, Shanghai Jiao Tong University 200240, P. R. China

Email: zhangsy16@sjtu.edu.cn

Email: zhuguodong1206@sjtu.edu.cn 


\section{Content}

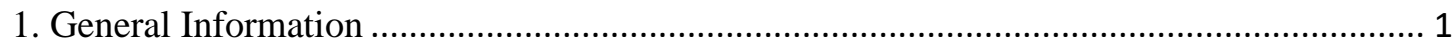

2. General Experimental Procedures for the Brönsted Acid Catalyzed Reactions ..................... 2

3. Experimental Procedures for the Transformation of 4ga. ................................................ 58

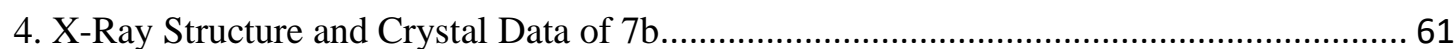

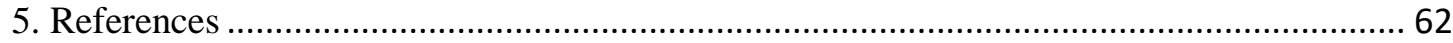

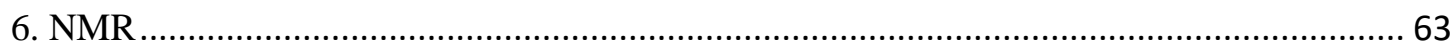




\section{General Information}

Instruments: Flash chromatography was carried out on 200-300 mesh silica gel, eluting with a mixture of petroleum ether (b.p. $60-90{ }^{\circ} \mathrm{C}$ ) and ethyl acetate. ${ }^{1} \mathrm{H}$ NMR and ${ }^{13} \mathrm{C}$ NMR spectra were acquired on a Bruker AVANCE III HD 400 spectrometer and Bruker AVANCE III HD 500 spectrometer, and calibrated using residual solvent peaks as internal reference. Enantiomeric excesses were measured on an Agilent HPLC. Optical rotations were measured on a Rudolph Research Analytical (Autopol VI). High resolution mass spectra (HRMS) were operated on a Bruker DaltonicsSolariX 7.0 Tesla Fourier Transform Ion Cyclotron Resonance (FT-ICR) Mass Spectrometer using the electrospray ionization (ESI) technique. Xray diffraction data were collected on a Bruker D8 Venture or a Bruker APEX-II CCD diffractometer. Melting points (m.p.) were measured on a SGW® X-4 digital melting point apparatus with microscope (Shanghai INESA Physical Optics Instrument Co., Ltd) and were uncorrected.

Reagents: All commercial materials were used as received unless otherwise noted. Phosphoric acid Organocatalysts ( $>98 \%, 98 \%$ ee, Daicel) were used without further purification. Solvents $\left(\mathrm{CH}_{3} \mathrm{CN}, \mathrm{DCM}\right.$, toluene, $\mathrm{Et}_{2} \mathrm{O}$, THF, et al) used in this manuscript were purchased from Energy in anhydrous form.

Substrate of indoles $\mathbf{2}$ were commercially available. Substrate of naphthylamines $\mathbf{1}$ could be prepared according to the literature procedure. ${ }^{1,2}$ 


\section{General Experimental Procedures for the Brönsted Acid Catalyzed Reactions}

Table S1. Optimization of Reaction Conditions ${ }^{a}$<smiles>C(#Cc1c(Nc2ccccc2)ccc2ccccc12)c1ccccc1</smiles>

$1 \mathrm{a}$<smiles>c1ccc2[nH]ccc2c1</smiles>
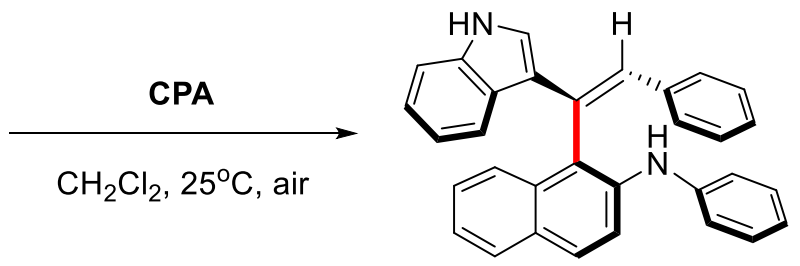

2a

3aa

\begin{tabular}{|l|l|l|l|l|l|l|}
\hline Entry & Cat. & Solvent & Additive & Yield \% & ee \% & E/Z \\
\hline 1 & CPA1 & DCM & none & 42 & -3 & $9: 1$ \\
\hline 2 & CPA2 & DCM & none & 59 & 17 & $7: 1$ \\
\hline 3 & CPA3 & DCM & none & 54 & 11 & $5: 1$ \\
\hline 4 & CPA4 & DCM & none & 62 & 15 & $4: 1$ \\
\hline 5 & CPA5 & DCM & none & 58 & 27 & $4: 1$ \\
\hline 6 & CPA6 & DCM & none & 66 & 24 & $2.5: 1$ \\
\hline 7 & CPA7 & DCM & none & 74 & 86 & $13: 1$ \\
\hline 8 & CPA7 & DCE & none & 70 & 86 & $>20: 1$ \\
\hline 9 & CPA7 & CHCl & none & 75 & 84 & $16: 1$ \\
\hline 10 & CPA7 & Toluene & none & 82 & 88 & $13: 1$ \\
\hline 11 & CPA7 & THF & none & $<2$ & - & - \\
\hline 12 & CPA7 & MeCN & none & 30 & 60 & $11: 1$ \\
\hline $13^{b c}$ & CPA7 & Toluene & $3 \AA$ MS & 87 & 89 & $16: 1$ \\
\hline $14^{b c}$ & CPA7 & Toluene & $4 \AA$ MS & 95 & 91 & $10: 1$ \\
\hline $15^{b c}$ & CPA7 & Toluene & $5 \AA$ MS & 94 & 90 & $10: 1$ \\
\hline $16^{b d}$ & CPA7 & Toluene & $4 \AA$ MS & 92 & 92 & $12: 1$ \\
\hline $17^{b d}$ & CPA7 & Toluene & $4 \AA$ MS & 90 & 94 & $12: 1$ \\
\hline & & & & & \\
\hline
\end{tabular}

${ }^{a}$ Reaction conditions: $1(0.05 \mathrm{mmol}), 2(0.1 \mathrm{mmol})$, catalyst $(10 \mathrm{~mol} \%)$ in Toluene $(0.5 \mathrm{~mL})$ at $25^{\circ} \mathrm{C}$ for $48 \mathrm{~h}$. Isolated yields were given. The ee values were determined by HPLC analysis using a chiral stationary phase. The Z/E values were determined by NMR analysis. ${ }^{b} 20{ }^{\circ} \mathrm{C} .{ }^{c}$ additive $(50 \mathrm{mg}) .{ }^{d}$ additive $(25 \mathrm{mg}) .{ }^{e}$ optimized conditions.<smiles>[R]c1cc2ccccc2c(-c2oc3cc4ccccc4cc([R])c2-3)c1[R]</smiles>

CPA1, R=H CPA2, $\mathrm{R}=\mathrm{Ph}$ CPA3, $\mathrm{R}=4-\mathrm{OMeC}_{6} \mathrm{H}_{4}$ CPA4, $\mathrm{R}=4-\mathrm{NO}_{2} \mathrm{C}_{6} \mathrm{H}_{4}$ CPA5, R=1-naphthaleny CPA6, R=9-phenanthreny CPA7, $\mathrm{R}=2,4,6-(\mathrm{iPr})_{3} \mathrm{C}_{6} \mathrm{H}_{2}$

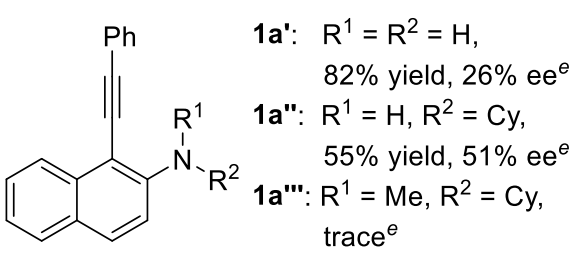

Procedure A (Experimental procedure for the synthesis of racemic product) 


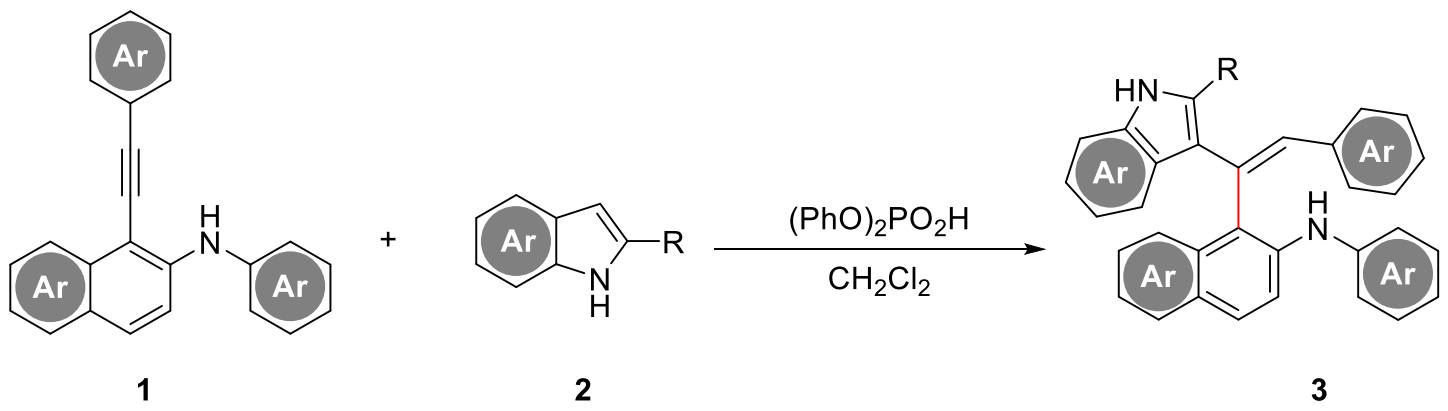

To a $10.0 \mathrm{~mL}$ reaction tube, $2(0.1 \mathrm{mmol}), 1(0.1 \mathrm{mmol}),(\mathrm{PhO})_{2} \mathrm{PO}_{2} \mathrm{H}(2.5 \mathrm{mg}, 0.01 \mathrm{mmol})$ and $1 \mathrm{~mL}$ DCM was added. The reaction mixture was stirred at ambient temperature and monitored by TLC. After completion of the reaction, the mixture was purified by preparative TLC on silica gel to yield the target molecular racemic product.

Procedure B (Experimental procedure for the synthesis of asymmetric product)
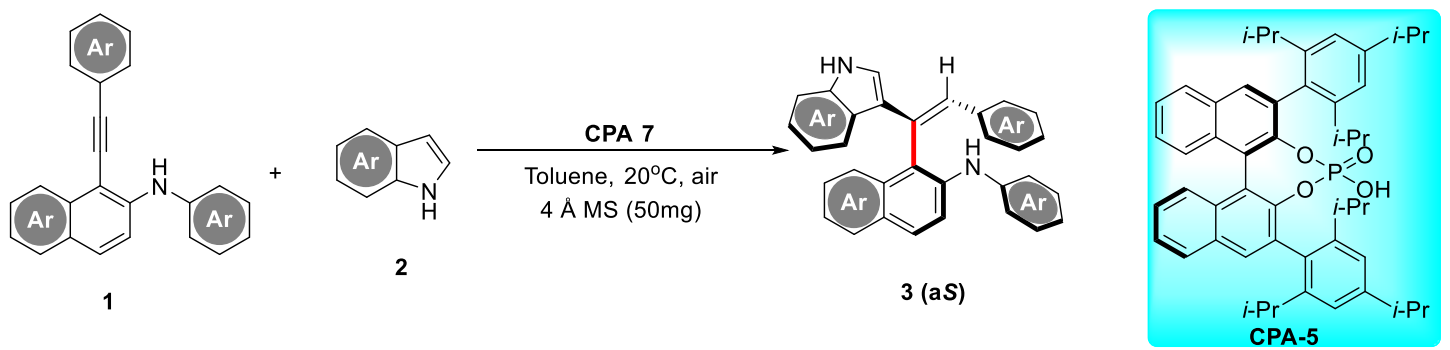

To a solution of $1(0.1 \mathrm{mmol})$, CPA7 $(7.5 \mathrm{mg}, 0.010 \mathrm{mmol})$ and $4 \AA \mathrm{MS}(50 \mathrm{mg})$ in toluene $(1 \mathrm{~mL}), 2$ $(0.15 \mathrm{mmol})$ was added in one portion at $20^{\circ} \mathrm{C}$ and monitored by TLC. After stirring for $48 \mathrm{~h}$, the mixture was directly purified through flash column chromatography on silica gel to give the pure product. Unless it was remarked, the temperature is $15^{\circ} \mathrm{C}$, and the solvent is $\mathrm{CH}_{2} \mathrm{Cl}_{2}$.

(aS)-(Z)-1-(1-(1H-indol-3-yl)-2-phenylvinyl)-N-phenylnaphthalen-2-amine (3aa)

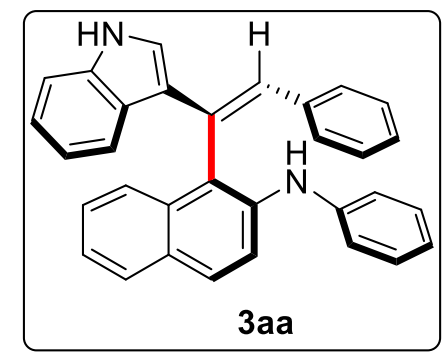

According to procedure B, 3aa was obtained as a white foam, $39.2 \mathrm{mg}$ (90\% yield, $94 \%$ ee, $\mathrm{Z}: \mathrm{E}=12: 1)$. ${ }^{1}$ H NMR (400 MHz, CDCl$) \delta 8.18(m, 1 H), 7.93(\mathrm{~s}, 1 \mathrm{H}), 7.78-7.75$ (m, 3H), 7.68 (dr, 1H), 7.55 (dd, $J$ $=8.9,1.9 \mathrm{~Hz}, 1 \mathrm{H}), 7.34-7.31(\mathrm{~m}, 1 \mathrm{H}), 7.27-7.22(\mathrm{~m}, 5 \mathrm{H}), 7.13(\mathrm{ddd}, J=8.9,7.1,1.9 \mathrm{~Hz}, 2 \mathrm{H}), 7.01-$ $7.00(\mathrm{~m}, 5 \mathrm{H}), 6.87-6.83(\mathrm{~m}, 3 \mathrm{H}), 6.67(\mathrm{~d}, J=2.7 \mathrm{~Hz}, 1 \mathrm{H}), 6.15(\mathrm{~s}, 1 \mathrm{H})$.

${ }^{13}$ C NMR (101 MHz, CDCl 3$) \delta 143.1,137.8,137.3,137.2,133.3,131.3,129.3,129.2,128.4,128.2$, $128.0,128.0,126.7,125.4,125.2,125.1,123.3,122.7,122.6,121.5,120.81,120.75,119.2,118.5,118.2$, 111.7 .

HRMS (ESI) calculated for $[\mathrm{M}+\mathrm{H}]{ }^{+} \mathrm{C}_{32} \mathrm{H}_{25} \mathrm{~N}_{2}{ }^{+}, \mathrm{m} / \mathrm{z}$ : 437.2018, found: 437.2015.

M.P. $74-76^{\circ} \mathrm{C}$.

$[\alpha]_{20 \mathrm{D}}=-39.6^{\circ}\left(\mathrm{c}=1, \mathrm{CHCl}_{3}\right)$.

HPLC analysis: HPLC DAICEL CHIRALPAK AD-H, hexane/isopropyl alcohol = 60/40, flow rate = 1 
$\mathrm{mL} / \mathrm{min}, \lambda=254 \mathrm{~nm}), \mathrm{tR}($ major $)=13.9 \mathrm{~min}, \mathrm{tR}($ minor $)=4.5 \mathrm{~min}$, ee $=94 \%$.

Chiral HPLC spectrum of racemic $3 a a$

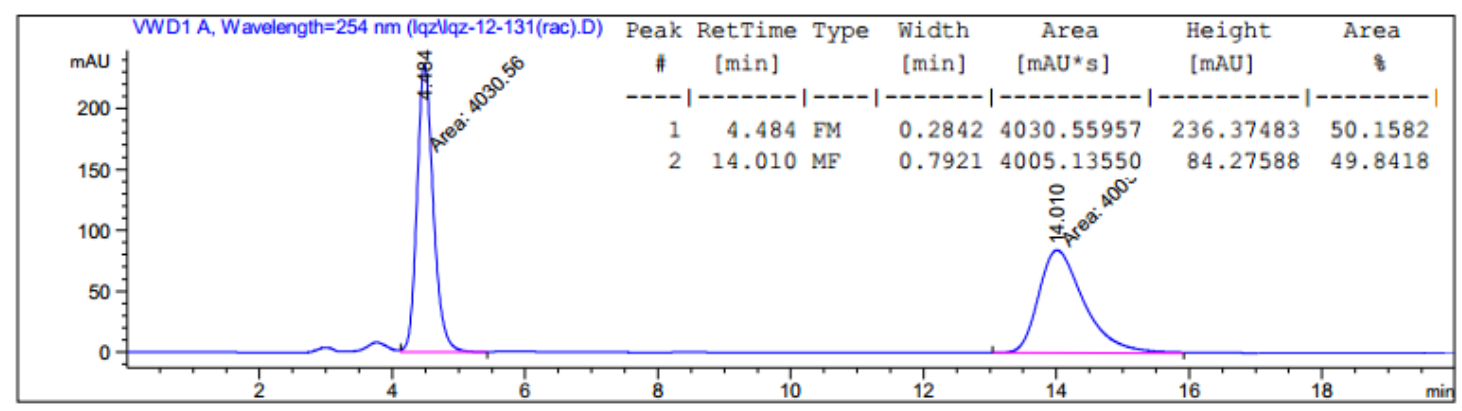

Chiral HPLC spectrum of $\mathbf{3 a a}$
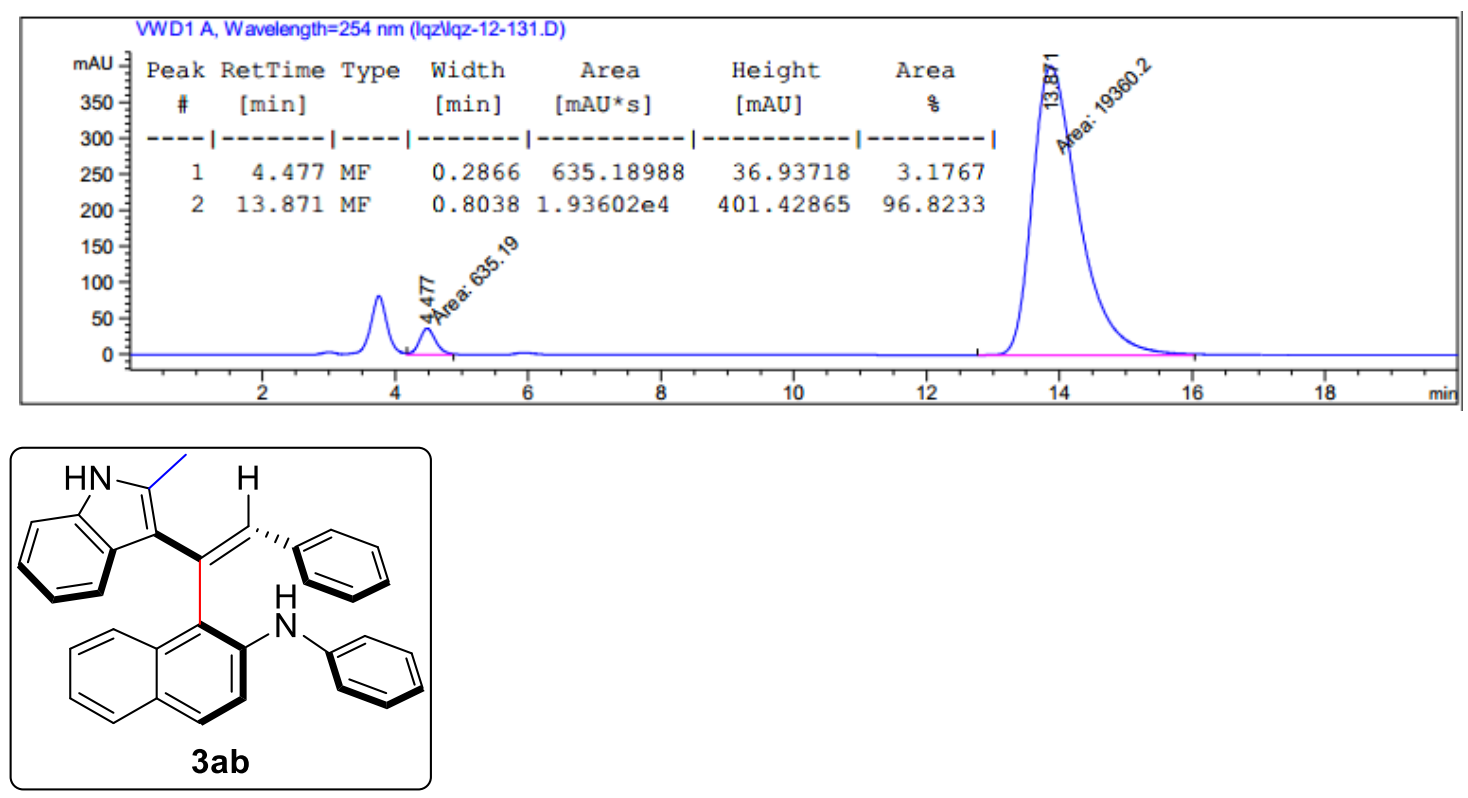

(aS)-(Z)-1-(1-(2-methyl-1H-indol-3-yl)-2-phenylvinyl)-N-phenylnaphthalen-2-amine (3ab)

According to procedure B, 3ab was obtained as a yellow foam, $27.9 \mathrm{mg}$ (62\% yield, 90\% ee, Z:E > 20:1). ${ }^{1} \mathbf{H}$ NMR (400 MHz, CDCl $)_{3} \delta 7.81-7.79(\mathrm{~m}, 1 \mathrm{H}), 7.75-7.68(\mathrm{~m}, 4 \mathrm{H}), 7.47$ (d, J= 8.9 Hz, 1H), 7.32 (s, 1H), $7.23-7.17(\mathrm{~m}, 3 \mathrm{H}), 7.14-7.09(\mathrm{~m}, 4 \mathrm{H}), 7.01(\mathrm{~s}, 5 \mathrm{H}), 6.85(\mathrm{t}, J=7.4 \mathrm{~Hz}, 1 \mathrm{H}), 6.76-6.74(\mathrm{~m}$, 2H), $6.28(\mathrm{~s}, 1 \mathrm{H}), 1.92(\mathrm{~s}, 3 \mathrm{H})$.

${ }^{13}$ C NMR (101 MHz, $\left.\mathbf{C D C l}_{3}\right) \delta$ 142.7, 138.0, 137.3, 135.2, 133.6, 133.5, 131.6, 131.3, 129.3, 129.1, $128.6,128.22$, 128.18, 128.1 , 127.8, 127.0, 126.6, 125.1, 123.2, 122.2, 121.6, 121.5, 120.3, 119.5, 119.1, $117.8,114.9,110.5,13.0$.

HRMS (ESI) calculated for $[\mathrm{M}+\mathrm{H}]^{+} \mathrm{C}_{33} \mathrm{H}_{27} \mathrm{~N}_{2}{ }^{+}, \mathrm{m} / \mathrm{z}: 451.2174$, found: 451.2171 .

M.P. $73-76^{\circ} \mathrm{C}$.

$[\alpha]^{20}{ }_{\mathrm{D}}=156^{\circ}\left(\mathrm{c}=0.2, \mathrm{CHCl}_{3}\right)$.

HPLC analysis: HPLC DAICEL CHIRALPAK AD-H, hexane/isopropyl alcohol = 90/10, flow rate = 1 $\mathrm{mL} / \mathrm{min}, \lambda=254 \mathrm{~nm})$, tR (major) $=23.7 \mathrm{~min}, \mathrm{tR}($ minor $)=11.0 \mathrm{~min}$, ee $=90 \%$.

Chiral HPLC spectrum of racemic $\mathbf{3 a b}$ 


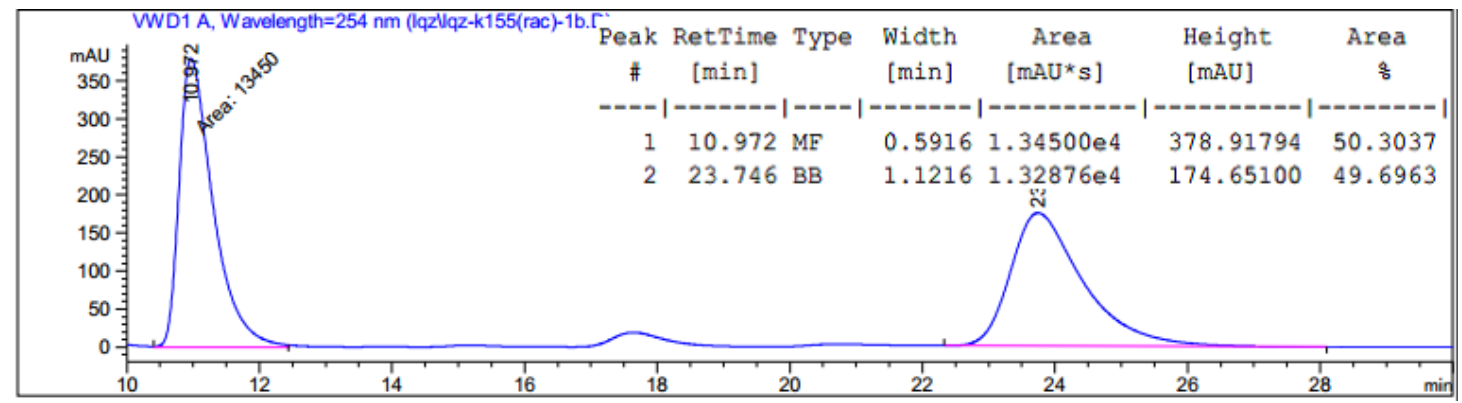

Chiral HPLC spectrum of $\mathbf{3 a b}$

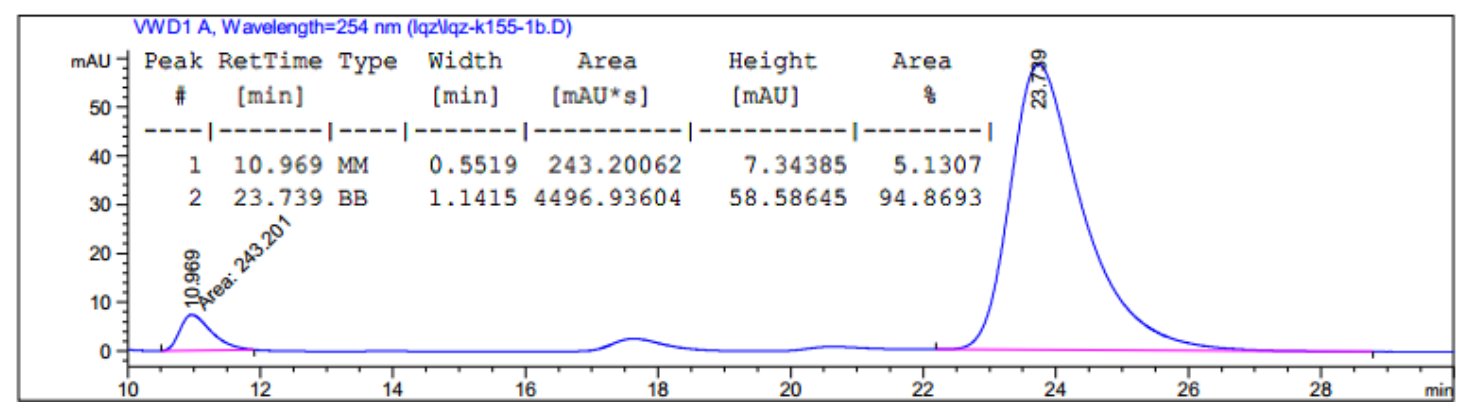

(aS)-(Z)-1-(1-(4-methyl-1H-indol-3-yl)-2-phenylvinyl)-N-phenylnaphthalen-2-amine (3ac)

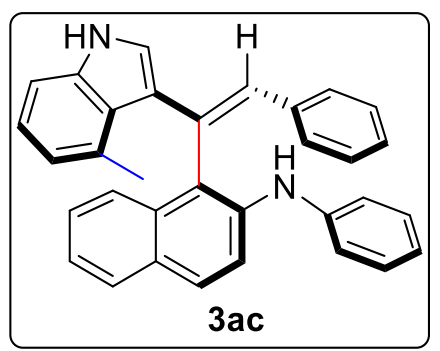

According to procedure $\mathbf{B}$, 3ac was obtained as a yellow foam, $27.9 \mathrm{mg}$ ( $62 \%$ yield, $94 \%$ ee, $\mathrm{Z}: \mathrm{E}=5: 1$ ). ${ }^{1}$ H NMR (400 MHz, CDCl $) \delta 8.02(\mathrm{~s}, 1 \mathrm{H}), 7.92-7.90(\mathrm{~m}, 1 \mathrm{H}), 7.77-7.73(\mathrm{~m}, 2 \mathrm{H}), 7.54(\mathrm{~d}, J=8.9$ $\mathrm{Hz}, 1 \mathrm{H}), 7.29-7.24(\mathrm{~m}, 3 \mathrm{H}), 7.21-7.12(\mathrm{~m}, 5 \mathrm{H}), 7.06-6.98(\mathrm{~m}, 6 \mathrm{H}), 6.88-6.82(\mathrm{~m}, 3 \mathrm{H}), 6.80(\mathrm{~d}, J$ $=2.8 \mathrm{~Hz}, 1 \mathrm{H}), 6.30(\mathrm{~s}, 1 \mathrm{H}), 2.90(\mathrm{~s}, 3 \mathrm{H})$.

${ }^{13} \mathbf{C}$ NMR (101 MHz, $\left.\mathbf{C D C l}_{3}\right) \delta 143.2,137.2,137.2,136.9,133.8,133.2,131.0,130.6,129.6,129.2$, 128.4, 128.3, 128.0, 126.9, 126.8, 125.3, 124.9, 124.6, 124.4, 123.4, 123.3, 122.6, 121.2, 120.8, 118.6, $118.5,109.3,22.5$.

HRMS (ESI) calculated for $[\mathrm{M}+\mathrm{H}]^{+} \mathrm{C}_{33} \mathrm{H}_{27} \mathrm{~N}_{2}{ }^{+}, \mathrm{m} / \mathrm{z}: 451.2174$, found: 451.2170 .

M.P. $77-79^{\circ} \mathrm{C}$.

$[\alpha]_{20 \mathrm{D}}=-32^{\circ}\left(\mathrm{c}=0.2, \mathrm{CHCl}_{3}\right)$.

HPLC analysis: HPLC DAICEL CHIRALPAK AD-H, hexane/isopropyl alcohol $=60 / 40$, flow rate $=1$ $\mathrm{mL} / \mathrm{min}, \lambda=254 \mathrm{~nm}), \mathrm{tR}($ major $)=45.49 \mathrm{~min}, \mathrm{tR}($ minor $)=15.1 \mathrm{~min}, \mathrm{ee}=94 \%$.

Chiral HPLC spectrum of racemic $3 a c$ 


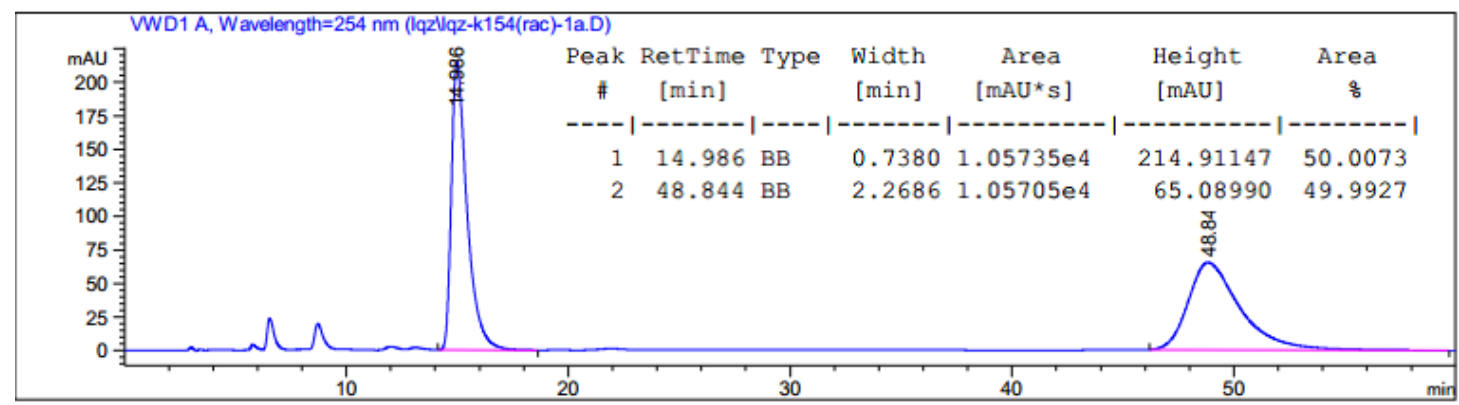

Chiral HPLC spectrum of $\mathbf{3 a c}$
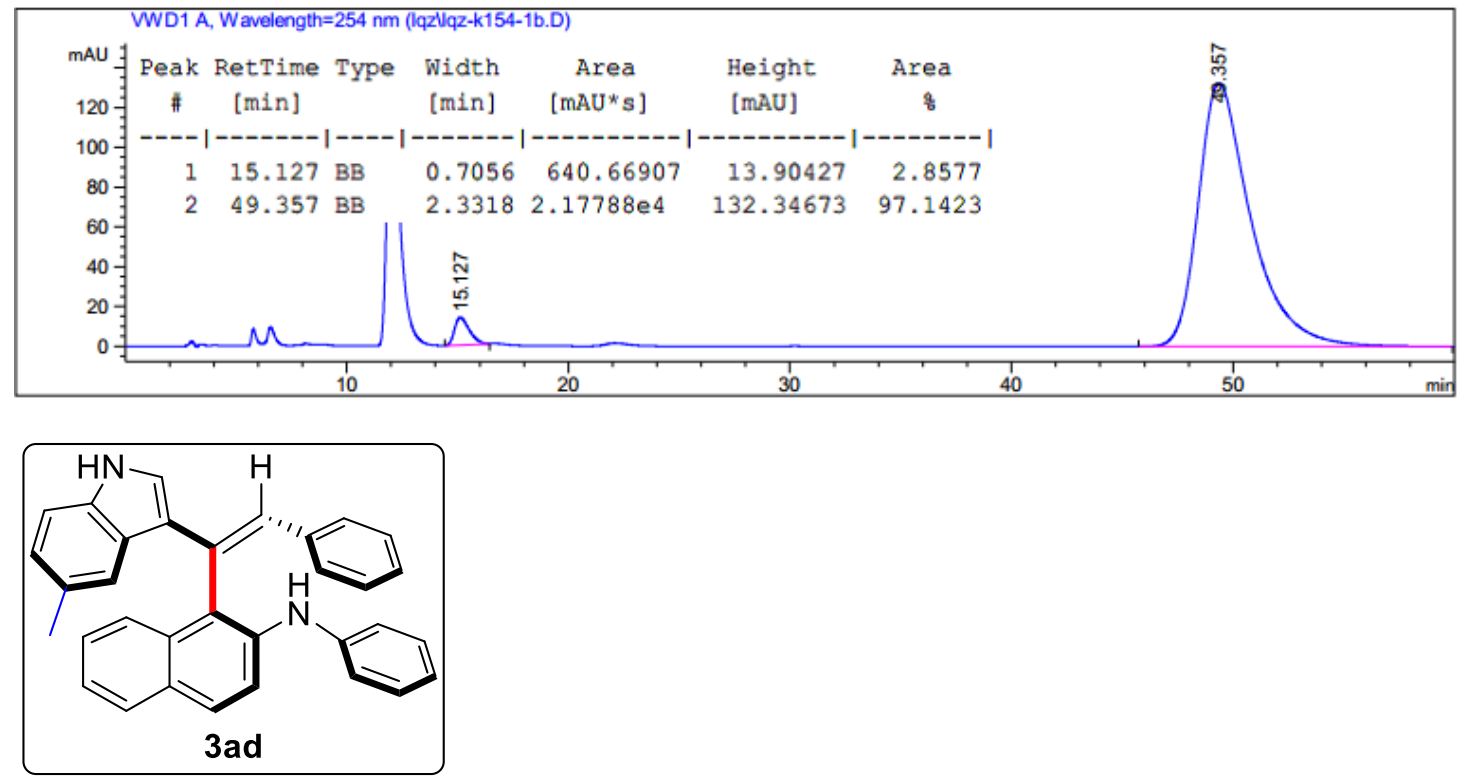

(aS)- (Z)-1-(1-(5-methyl-1H-indol-3-yl)-2-phenylvinyl)-N-phenylnaphthalen-2-amine (3ad)

According to procedure $\mathbf{B}, 3$ ad was obtained as a yellow foam, $34.2 \mathrm{mg}$ (76\% yield, $92 \%$ ee, $\mathrm{Z}: \mathrm{E}=6: 1$ ). ${ }^{1}$ H NMR (500 MHz, CDCl $) \delta 8.05(\mathrm{~s}, 1 \mathrm{H}), 7.92(\mathrm{br}, 1 \mathrm{H}), 7.82-7.79(\mathrm{~m}, 3 \mathrm{H}), 7.71(\mathrm{~s}, 1 \mathrm{H}), 7.59(\mathrm{~d}, J$ $=9.0 \mathrm{~Hz}, 1 \mathrm{H}), 7.30-7.28(\mathrm{~m}, 3 \mathrm{H}), 7.19-7.16(\mathrm{~m}, 2 \mathrm{H}), 7.13(\mathrm{dd}, J=8.3,1.6 \mathrm{~Hz}, 1 \mathrm{H}), 7.07-7.03(\mathrm{~m}$, $5 \mathrm{H}), 6.90-6.88(\mathrm{~m}, 3 \mathrm{H}), 6.67$ (d, $J=2.7 \mathrm{~Hz}, 1 \mathrm{H}), 6.20(\mathrm{~s}, 1 \mathrm{H}), 2.59(\mathrm{~s}, 3 \mathrm{H})$.

${ }^{13} \mathbf{C}$ NMR (126 MHz, $\left.\mathbf{C D C l}_{3}\right) \delta 143.1,137.7,137.3,135.6,133.3,131.4,130.1,129.3,129.1,128.4$, $128.2,127.9,127.8,126.7,126.6,125.6,125.33,125.24,124.2,123.3,122.8,121.5,120.6,119.2,118.2$, 118.0, 111.3, 21.9.

HRMS (ESI) calculated for $[\mathrm{M}+\mathrm{H}]^{+} \mathrm{C}_{33} \mathrm{H}_{27} \mathrm{~N}_{2}{ }^{+}, \mathrm{m} / \mathrm{z}: 451.2174$, found: 451.2173 .

M.P. $80-83^{\circ} \mathrm{C}$.

$[\alpha]_{20} \mathrm{D}=-27^{\circ}\left(\mathrm{c}=0.2, \mathrm{CHCl}_{3}\right)$.

HPLC analysis: HPLC DAICEL CHIRALPAK AD-H, hexane/isopropyl alcohol $=80 / 20$, flow rate $=1$ $\mathrm{mL} / \mathrm{min}, \lambda=254 \mathrm{~nm}$ ), $\operatorname{tr}($ major $)=16.6 \mathrm{~min}, \operatorname{tr}(\operatorname{minor})=5.9 \mathrm{~min}$, ee $=92 \%$.

Chiral HPLC spectrum of racemic 3 ad 


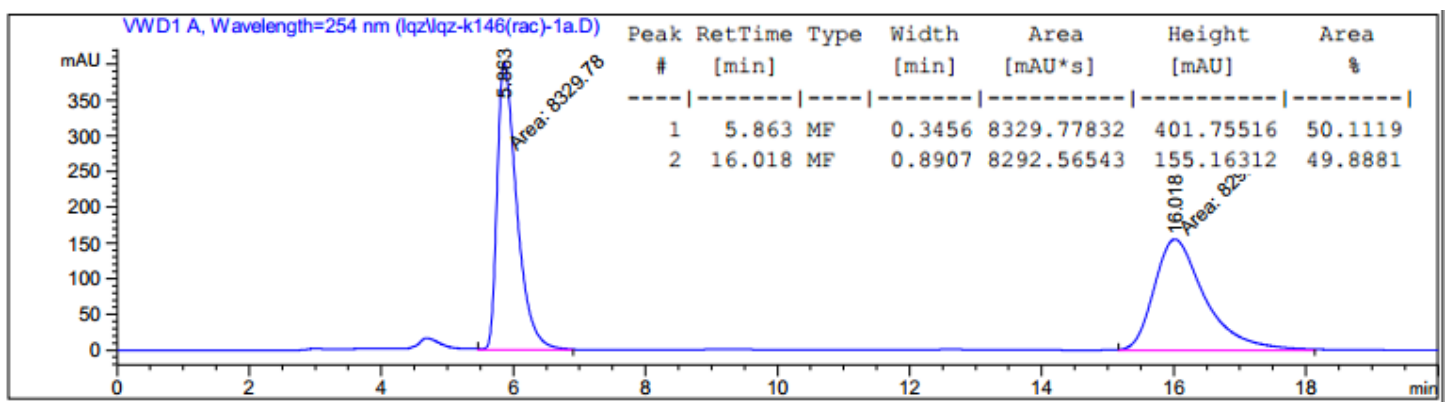

Chiral HPLC spectrum of $\mathbf{3 a d}$
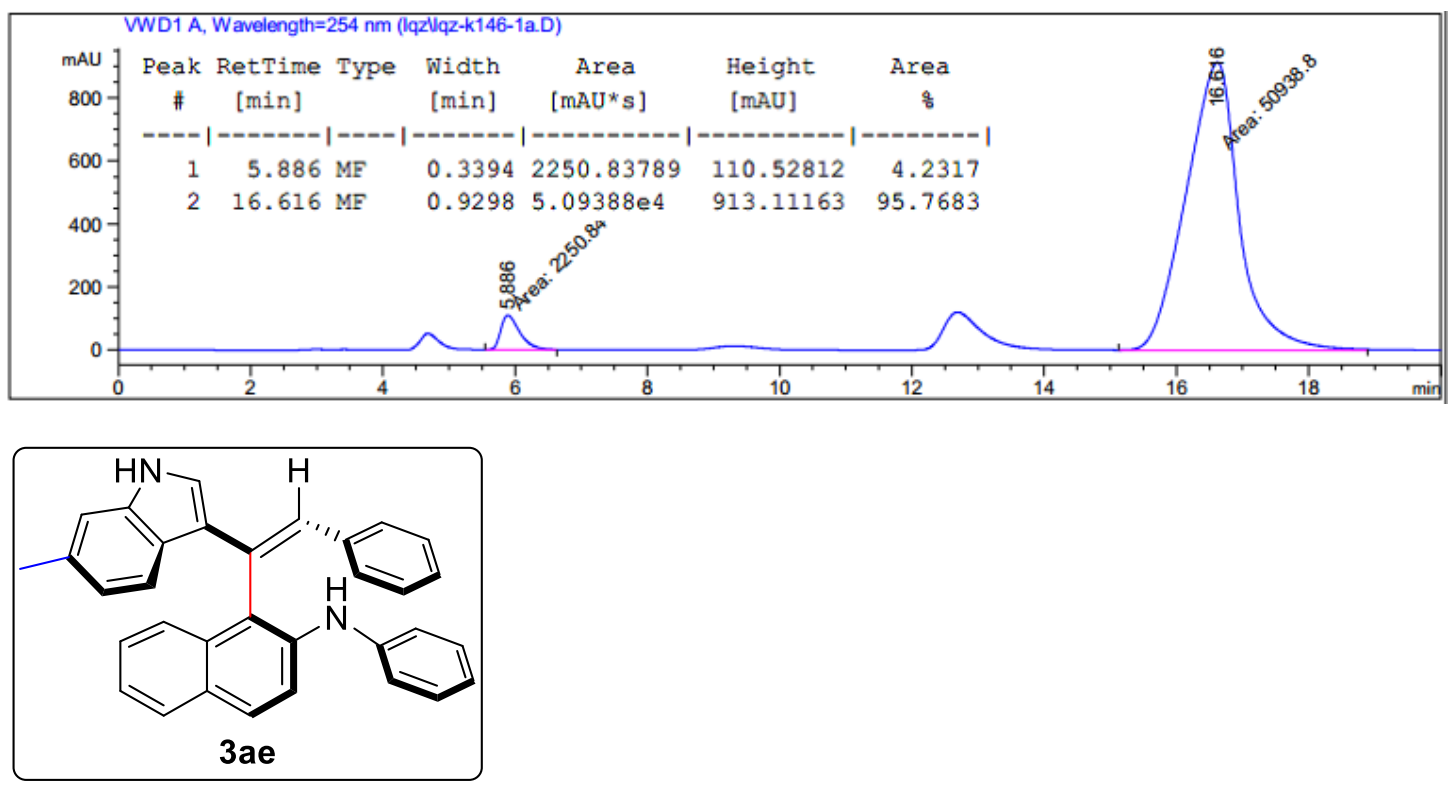

(aS)-(Z)-1-(1-(6-methyl-1H-indol-3-yl)-2-phenylvinyl)-N-phenylnaphthalen-2-amine (3ae) According to procedure $\mathbf{B}$ under $10{ }^{\circ} \mathrm{C}$, 3ae was obtained as a yellow oil, $31.5 \mathrm{mg}$ (70\% yield, $95 \%$ ee, $\mathrm{Z}: \mathrm{E}=12: 1)$.

${ }^{1} \mathbf{H}$ NMR (600 MHz, CDCl$) \delta 8.06(\mathrm{~d}, J=8.2 \mathrm{~Hz}, 1 \mathrm{H}), 7.87(\mathrm{~s}, 1 \mathrm{H}), 7.78-7.75(\mathrm{~m}, 3 \mathrm{H}), 7.66(\mathrm{~s}, 1 \mathrm{H})$, $7.55(\mathrm{~d}, J=8.9 \mathrm{~Hz}, 1 \mathrm{H}), 7.27-7.22(\mathrm{~m}, 2 \mathrm{H}), 7.15-7.10(\mathrm{~m}, 4 \mathrm{H}), 7.02-6.98(\mathrm{~m}, 5 \mathrm{H}), 6.87-6.84(\mathrm{~m}$, $3 \mathrm{H}), 6.62(\mathrm{~d}, J=2.6 \mathrm{~Hz}, 1 \mathrm{H}), 6.15(\mathrm{~s}, 1 \mathrm{H}), 2.48(\mathrm{~s}, 3 \mathrm{H})$.

${ }^{13}$ C NMR (151 MHz, CDCl $)$ ) $\delta$ 143.1, 137.78, 137.76, 137.3, 133.3, 132.6, 131.3, 129.3, 129.1, 128.4, $128.2,127.9,127.7,126.7,126.6,125.2,124.9,123.3,122.9,122.7,122.4,121.5,120.5,119.2,118.3$, 118.1, 111.6, 21.6.

HRMS (ESI) calculated for $[\mathrm{M}+\mathrm{H}]^{+} \mathrm{C}_{33} \mathrm{H}_{27} \mathrm{~N}_{2}{ }^{+}, \mathrm{m} / \mathrm{z}: 451.2174$, found: 451.2154 .

$[\alpha]_{20 \mathrm{D}}=-28^{\circ}\left(\mathrm{c}=0.2, \mathrm{CHCl}_{3}\right)$.

HPLC analysis: HPLC DAICEL CHIRALPAK AD-H, hexane/isopropyl alcohol $=60 / 40$, flow rate $=1$ $\mathrm{mL} / \mathrm{min}, \lambda=365 \mathrm{~nm})$, tR (major) $=16.6 \mathrm{~min}, \mathrm{tR}($ minor $)=5.0 \mathrm{~min}$, ee $=95 \%$.

Chiral HPLC spectrum of racemic $3 a e$ 


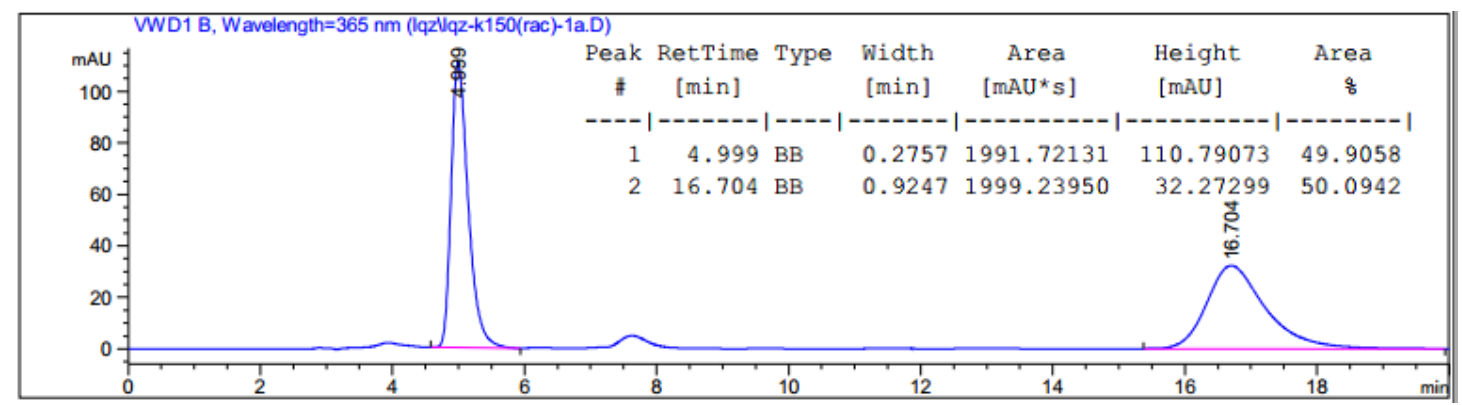

Chiral HPLC spectrum of $\mathbf{3 a e}$
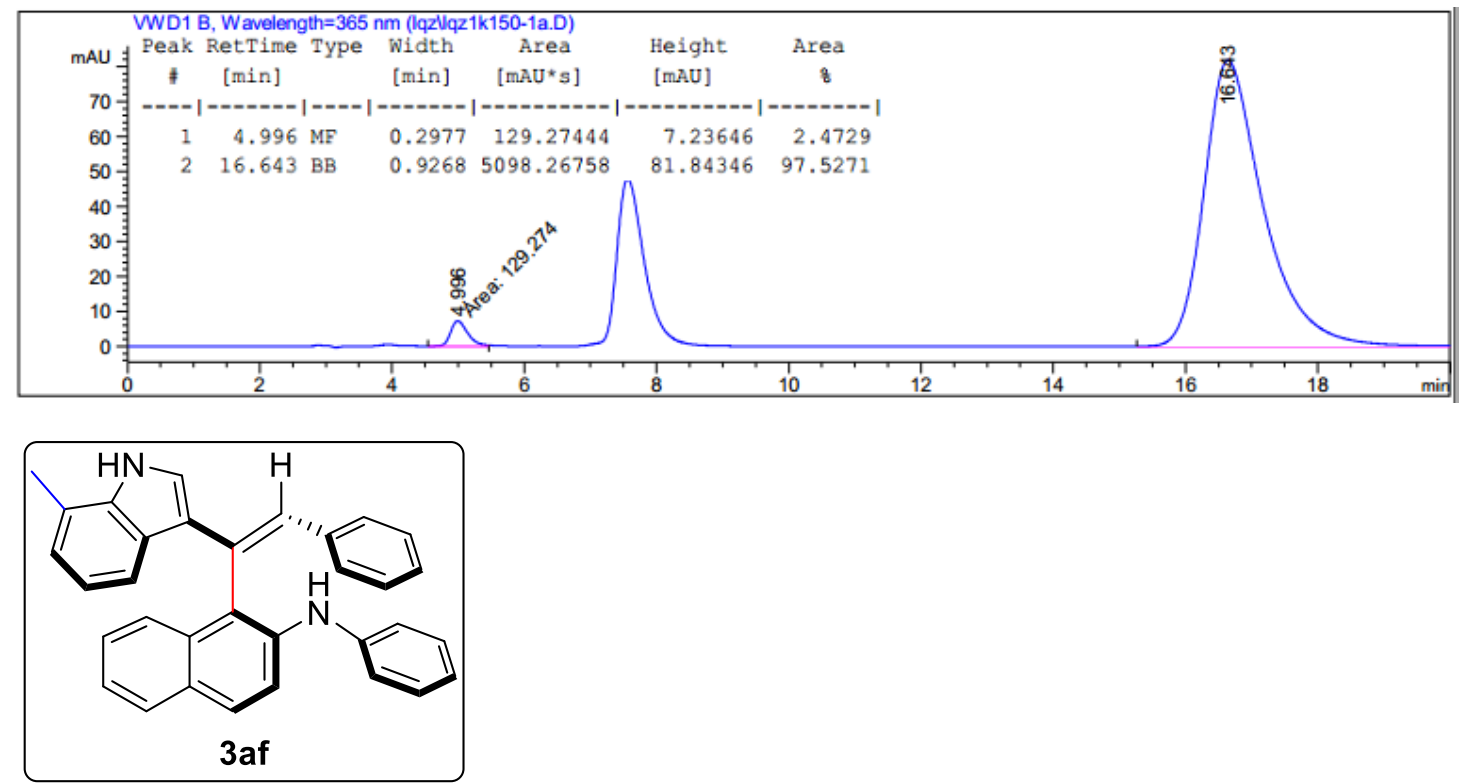

(aS)-(Z)-1-(1-(7-methyl-1H-indol-3-yl)-2-phenylvinyl)-N-phenylnaphthalen-2-amine (3af)

According to procedure $\mathbf{B}$, 3af was obtained as a yellow foam, $36.0 \mathrm{mg}$ ( $80 \%$ yield, $90 \%$ ee, $\mathrm{Z}: \mathrm{E}=19: 1)$. ${ }^{1} \mathbf{H}$ NMR (500 MHz, CDCl $) \delta 8.09(\mathrm{~d}, J=8.1 \mathrm{~Hz}, 1 \mathrm{H}), 7.89(\mathrm{~s}, 1 \mathrm{H}), 7.83-7.80(\mathrm{~m}, 3 \mathrm{H}), 7.73(\mathrm{~s}, 1 \mathrm{H})$, $7.61(\mathrm{~d}, J=8.9 \mathrm{~Hz}, 1 \mathrm{H}), 7.32-7.27(\mathrm{~m}, 2 \mathrm{H}), 7.25(\mathrm{t}, J=7.6 \mathrm{~Hz}, 1 \mathrm{H}), 7.18(\mathrm{t}, J=7.8 \mathrm{~Hz}, 2 \mathrm{H}), 7.12$ (d, $J=7.1 \mathrm{~Hz}, 1 \mathrm{H}), 7.09-7.02(\mathrm{~m}, 5 \mathrm{H}), 6.90(\mathrm{~d}, J=7.5 \mathrm{~Hz}, 3 \mathrm{H}), 6.70(\mathrm{~d}, J=2.7 \mathrm{~Hz}, 1 \mathrm{H}), 6.20(\mathrm{~s}, 1 \mathrm{H})$, $2.46(\mathrm{~s}, 3 \mathrm{H})$.

${ }^{13} \mathbf{C}$ NMR (126 MHz, CDCl $) \delta$ 143.0, 137.7, 137.2, 136.7, 133.2, 131.3, 129.2, 129.1, 128.3, 128.18, 128.16, 127.92, 127.89, 126.7, 126.6, 125.2, 125.1, 124.6, 123.3, 123.2, 122.8, 121.4, 120.9, 120.7, 1191, $118.9,118.5,118.2,16.5$.

HRMS (ESI) calculated for $[\mathrm{M}+\mathrm{H}]^{+} \mathrm{C}_{33} \mathrm{H}_{27} \mathrm{~N}_{2}{ }^{+}, \mathrm{m} / \mathrm{z}: 451.2174$, found: 451.2170 .

M.P. $78-80^{\circ} \mathrm{C}$.

$[\alpha]^{20}{ }_{\mathrm{D}}=-27^{\circ}\left(\mathrm{c}=0.2, \mathrm{CHCl}_{3}\right)$.

HPLC analysis: HPLC DAICEL CHIRALPAK AD-H, hexane/isopropyl alcohol $=80 / 20$, flow rate $=1$ $\mathrm{mL} / \mathrm{min}, \lambda=360 \mathrm{~nm}$ ), $\mathrm{tR}$ (major) $=9.6 \mathrm{~min}, \mathrm{tR}($ minor $)=5.4 \mathrm{~min}$, ee $=90 \%$.

Chiral HPLC spectrum of racemic $3 a f$ 


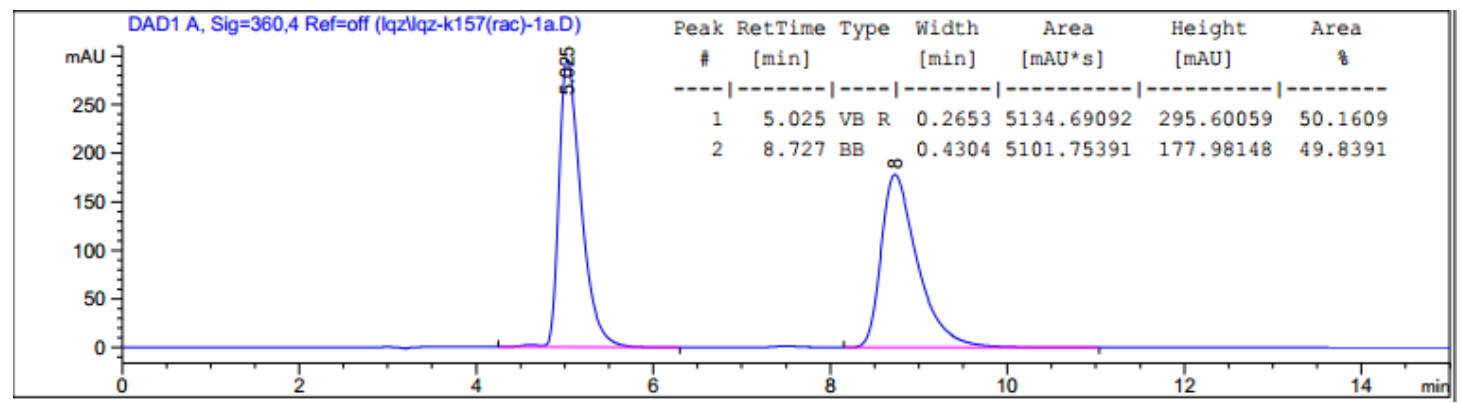

Chiral HPLC spectrum of $3 a f$

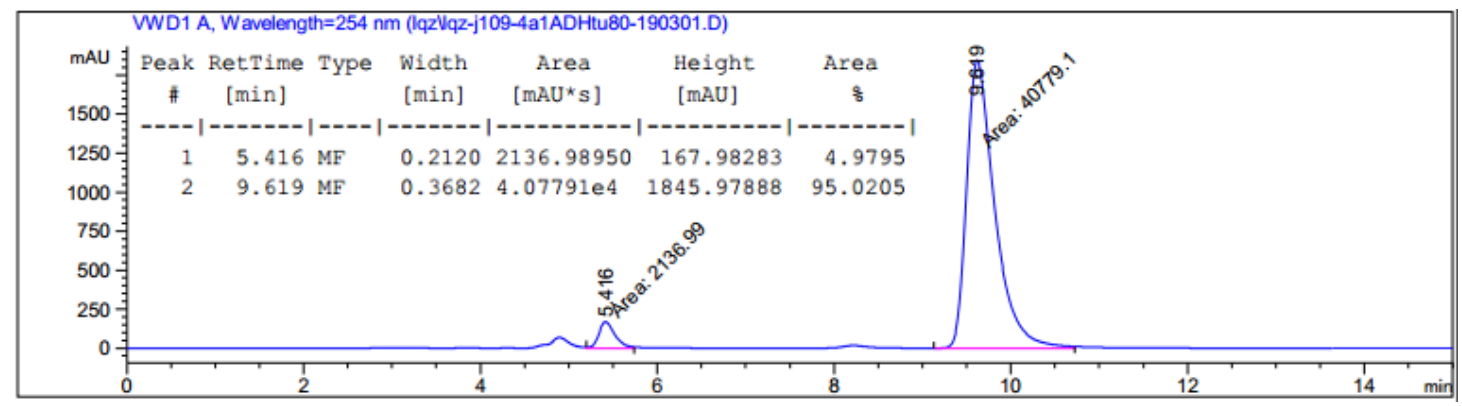

(aS)- (Z)-1-(1-(4-fluoro-1H-indol-3-yl)-2-phenylvinyl)-N-phenylnaphthalen-2-amine (3ag)

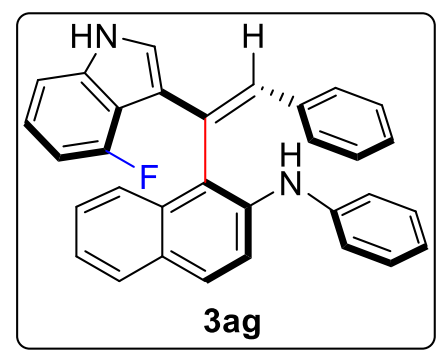

According to procedure $\mathbf{B}, \mathbf{3 a g}$ was obtained as a yellow foam, $31.3 \mathrm{mg}$ (69\% yield, $90 \%$ ee, $\mathrm{Z}: \mathrm{E}=14: 1)$. ${ }^{1} \mathbf{H}$ NMR (500 MHz, CDCl 3$) \delta 8.02(\mathrm{~s}, 1 \mathrm{H}), 7.87-7.85(\mathrm{~m}, 1 \mathrm{H}), 7.80-7.77(\mathrm{~m}, 2 \mathrm{H}), 7.72(\mathrm{~d}, J=2.0$ $\mathrm{Hz}, 1 \mathrm{H}), 7.58(\mathrm{~d}, J=9.0 \mathrm{~Hz}, 1 \mathrm{H}), 7.30-7.28(\mathrm{~m}, 2 \mathrm{H}), 7.20-7.15(\mathrm{~m}, 3 \mathrm{H}), 7.10(\mathrm{~d}, J=8.0 \mathrm{~Hz}, 1 \mathrm{H})$, $7.04-7.00(\mathrm{~m}, 5 \mathrm{H}), 6.98-6.92(\mathrm{~m}, 1 \mathrm{H}), 6.91-6.86(\mathrm{~m}, 3 \mathrm{H}), 6.63(\mathrm{~d}, J=2.6 \mathrm{~Hz}, 1 \mathrm{H}), 6.26(\mathrm{~s}, 1 \mathrm{H})$.

${ }^{13} \mathbf{C}$ NMR (126 MHz, $\left.\mathbf{C D C l}_{3}\right) \delta 157.9,155.9,143.0,139.8,139.7,137.5,137.2,133.1,131.7,131.6$, $129.3,129.1,128.8,128.29,128.25,128.1,127.9,126.8,126.8,125.4,125.3,123.31,123.27,123.22$, 123.20, 121.4, 119.0, 118.63, 118.60, 118.1, 113.8, 113.6, 107.6, 106.4, 106.2.

HRMS (ESI) calculated for $[\mathrm{M}+\mathrm{H}]^{+} \mathrm{C}_{32} \mathrm{H}_{24} \mathrm{FN}_{2}{ }^{+}, \mathrm{m} / \mathrm{z}: 455.1924$, found: 455.1925 .

M.P. $95-97^{\circ} \mathrm{C}$.

$[\alpha]_{20 \mathrm{D}}=-54^{\circ}\left(\mathrm{c}=0.2, \mathrm{CHCl}_{3}\right)$.

HPLC analysis: HPLC DAICEL CHIRALPAK AD-H, hexane/isopropyl alcohol = 70/30, flow rate = 1 $\mathrm{mL} / \mathrm{min}, \lambda=365 \mathrm{~nm}$ ), tR (major) $=9.4 \mathrm{~min}, \mathrm{tR}($ minor $)=4.8 \mathrm{~min}$, ee $=90 \%$.

Chiral HPLC spectrum of racemic $3 a g$ 


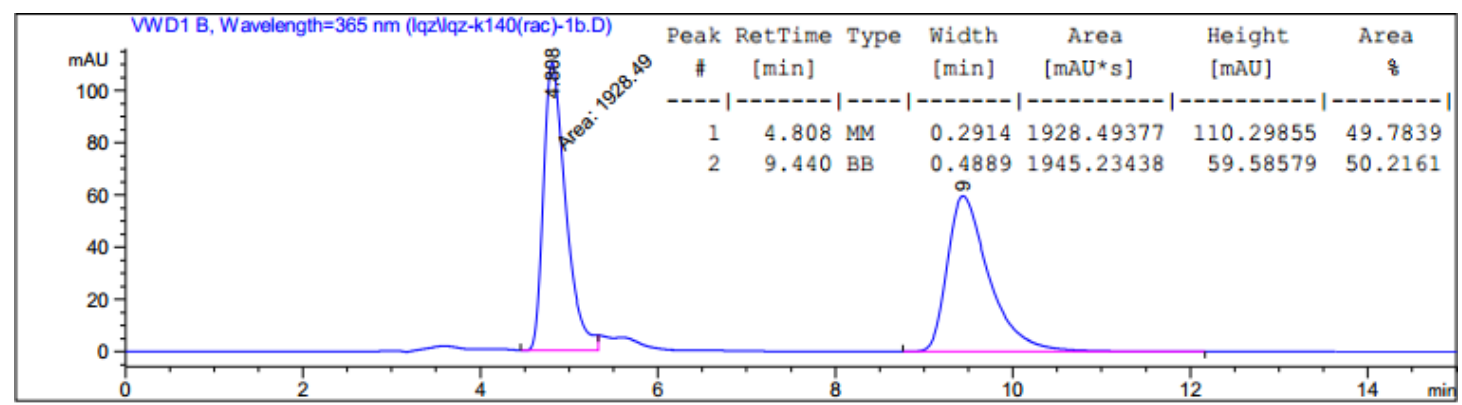

Chiral HPLC spectrum of $\mathbf{3 a g}$
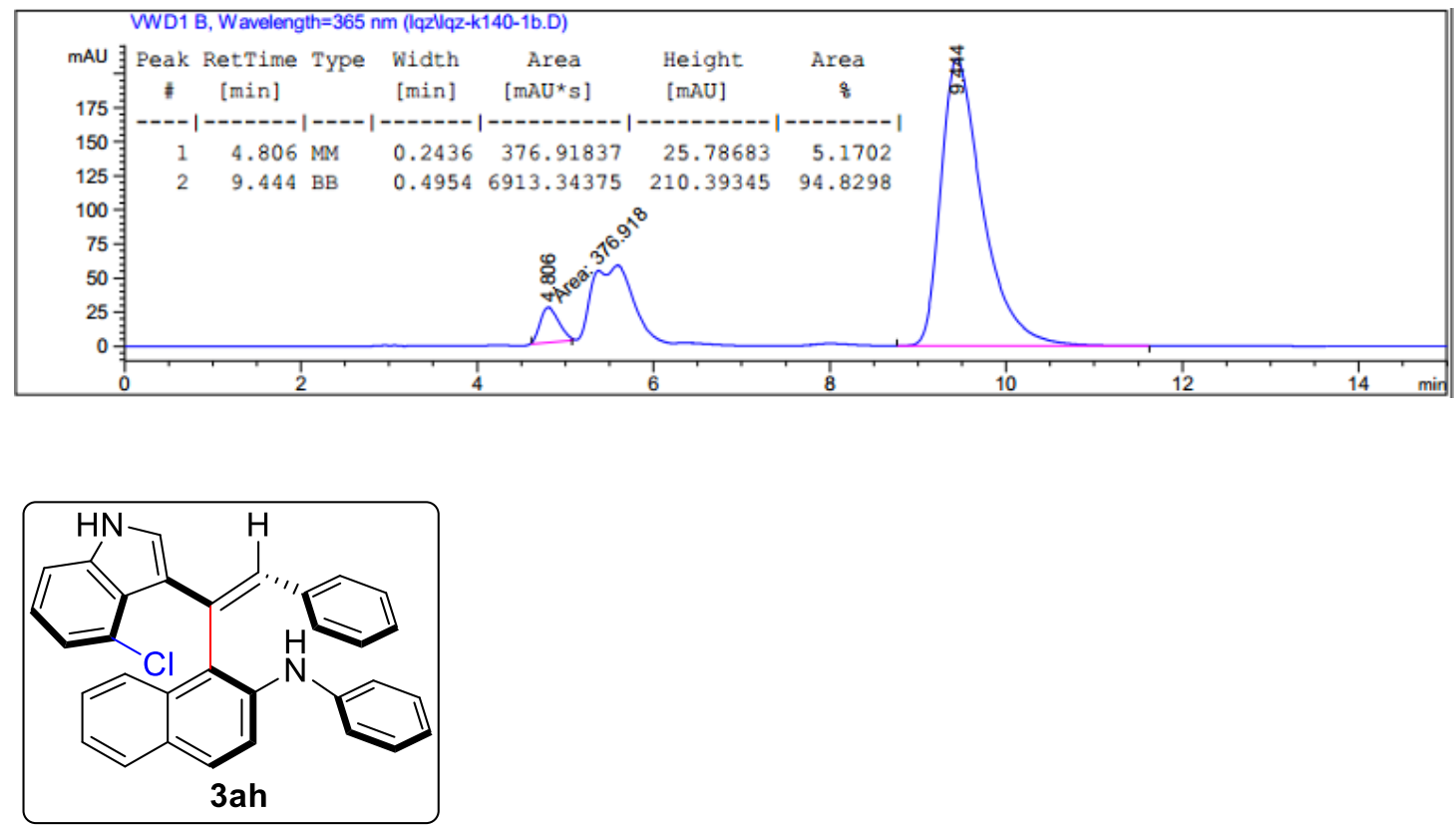

(aS)- (Z)-1-(1-(4-chloro-1H-indol-3-yl)-2-phenylvinyl)-N-phenylnaphthalen-2-amine (3ah)

According to procedure $\mathbf{B}, \mathbf{3 a h}$ was obtained as a yellow foam, $41.9 \mathrm{mg}$ ( $89 \%$ yield, $91 \%$ ee, $\mathrm{Z}: \mathrm{E}=9: 1)$. ${ }^{1}$ H NMR (500 MHz, CDCl $) \delta 8.02(\mathrm{~d}, J=8.3 \mathrm{~Hz}, 1 \mathrm{H}), 7.97$ (br, 1H), $7.75(\mathrm{t}, J=7.9 \mathrm{~Hz}, 2 \mathrm{H}), 7.59(\mathrm{~d}$, $J=8.8 \mathrm{~Hz}, 1 \mathrm{H}), 7.32-7.29(\mathrm{~m}, 2 \mathrm{H}), 7.24-7.21(\mathrm{~m}, 2 \mathrm{H}), 7.16-7.10$ (m, 4H), 7.03 (br, 5H), 6.91 (d, $J$ $=8.2 \mathrm{~Hz}, 2 \mathrm{H}), 6.85-6.82(\mathrm{~m}, 1 \mathrm{H}), 6.77(\mathrm{~d}, J=2.1 \mathrm{~Hz}, 1 \mathrm{H}), 6.53(\mathrm{~s}, 1 \mathrm{H})$.

${ }^{13}$ C NMR (126 MHz, $\left.\mathbf{C D C l}_{3}\right) \delta 143.0,137.9,137.2,136.8,136.7,132.8,129.3,129.1,128.3,128.19$, $128.17,128.0,127.1,127.0,126.9,126.3,125.6,125.1,124.4,123.3,122.8,122.6,122.4,121.3,119.98$, $119.97,118.5,118.2,110.3$.

HRMS (ESI) calculated for $[\mathrm{M}+\mathrm{H}]^{+} \mathrm{C}_{32} \mathrm{H}_{24} \mathrm{ClN}_{2}{ }^{+}, \mathrm{m} / \mathrm{z}$ : 471.1628, found: 471.1629 .

M.P. $80-83^{\circ} \mathrm{C}$.

$[\alpha]_{20 \mathrm{D}}=-183^{\circ}\left(\mathrm{c}=0.2, \mathrm{CHCl}_{3}\right)$.

HPLC analysis: HPLC DAICEL CHIRALPAK AD-H, hexane/isopropyl alcohol $=90 / 10$, flow rate $=1$ $\mathrm{mL} / \mathrm{min}, \lambda=254 \mathrm{~nm}$ ), $\mathrm{tR}($ major $)=32.6 \mathrm{~min}, \mathrm{tR}(\operatorname{minor})=16.8 \mathrm{~min}, \mathrm{ee}=91 \%$.

Chiral HPLC spectrum of racemic 3 ah 


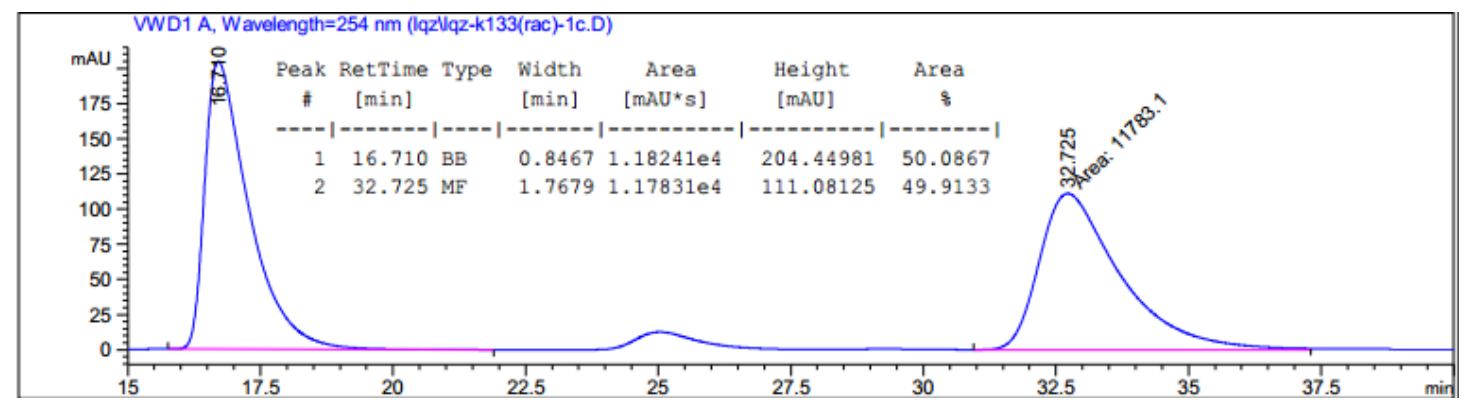

Chiral HPLC spectrum of $\mathbf{3 a h}$
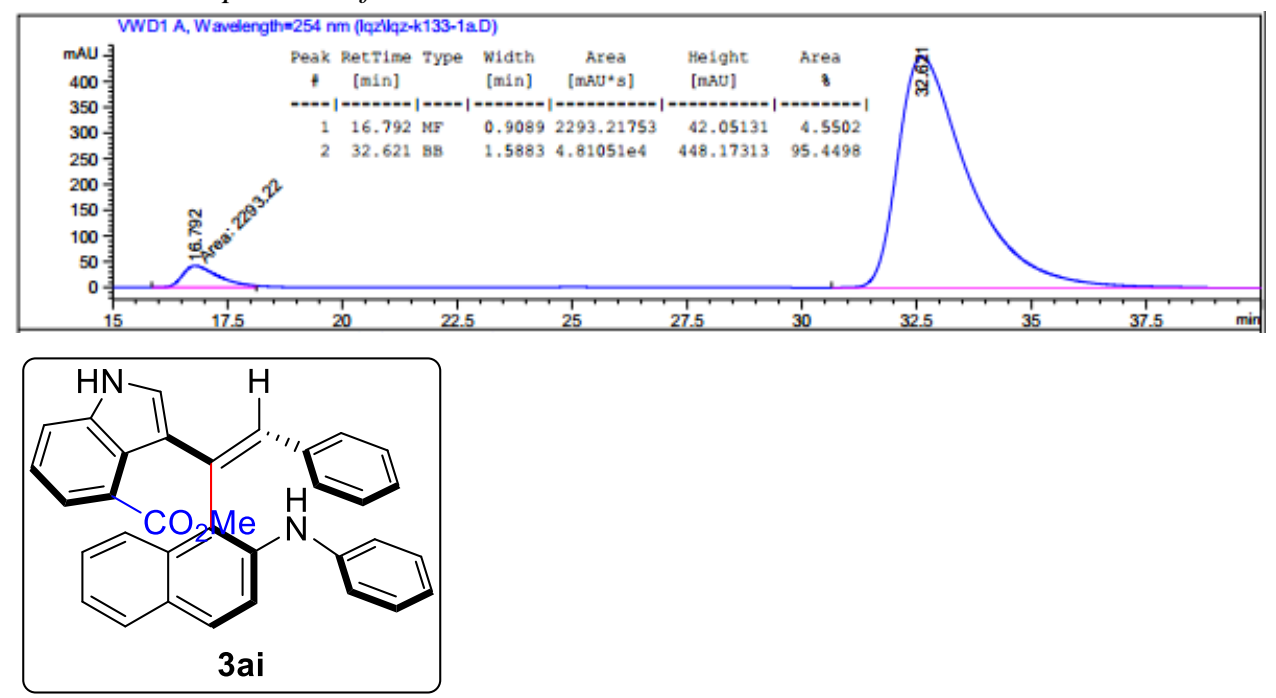

(aS)-methyl (Z)-3-(2-phenyl-1-(2-(phenylamino)naphthalen-1-yl)vinyl)-1H-indole-4carboxylate (3ai)

According to procedure $\mathbf{B}$, 3ai was obtained as a white foam, $46.9 \mathrm{mg}$ (95\% yield, $82 \%$ ee, $\mathrm{Z}: \mathrm{E}=16: 1)$. ${ }^{1}$ H NMR (500 MHz, $\left.\mathbf{C D C l}_{3}\right) \delta 8.18(\mathrm{br}, 1 \mathrm{H}), 7.90-7.88(\mathrm{~m}, 1 \mathrm{H}), 7.79-7.77(\mathrm{~m}, 2 \mathrm{H}), 7.69(\mathrm{~d}, J=9.0$ $\mathrm{Hz}, 1 \mathrm{H}), 7.60-7.59(\mathrm{~m}, 1 \mathrm{H}), 7.41(\mathrm{~d}, J=8.1 \mathrm{~Hz}, 1 \mathrm{H}), 7.34(\mathrm{~s}, 1 \mathrm{H}), 7.26-7.22(\mathrm{~m}, 3 \mathrm{H}), 7.14-7.11(\mathrm{~m}$, 2H), $7.02-6.97(\mathrm{~m}, 5 \mathrm{H}), 6.96-6.94(\mathrm{~m}, 2 \mathrm{H}), 6.83-6.80(\mathrm{~m}, 2 \mathrm{H}), 6.70(\mathrm{~d}, J=2.7 \mathrm{~Hz}, 1 \mathrm{H}), 3.71(\mathrm{~s}$, $3 \mathrm{H})$.

${ }^{13}$ C NMR (126 MHz, CDCl $) \delta$ ) 169.6, 143.2, 138.9, 136.9, 132.6, 131.7, 129.1, 128.9, 128.8, 128.18, $128.15,128.1,127.8,126.8,126.6,126.3,125.2,124.7,123.1,122.9,122.5,122.2,121.6,120.9,120.1$, $118.8,118.0,115.0,52.7$.

HRMS (ESI) calculated for $[\mathrm{M}+\mathrm{H}]^{+} \mathrm{C}_{34} \mathrm{H}_{27} \mathrm{~N}_{2} \mathrm{O}_{2}{ }^{+}, \mathrm{m} / \mathrm{z}$ : 495.2073, found: 495.2071 .

M.P. $109-111^{\circ} \mathrm{C}$.

$[\alpha]_{20} \mathrm{D}=-211^{\circ}\left(\mathrm{c}=0.2, \mathrm{CHCl}_{3}\right)$.

HPLC analysis: HPLC DAICEL CHIRALPAK OD-H, hexane/isopropyl alcohol $=80 / 20$, flow rate $=1$ $\mathrm{mL} / \mathrm{min}, \lambda=254 \mathrm{~nm}$ ), $\mathrm{tR}$ (major) $=6.3 \mathrm{~min}, \mathrm{tR}($ minor $)=8.3 \mathrm{~min}$, ee $=82 \%$.

Chiral HPLC spectrum of racemic $3 a i$ 


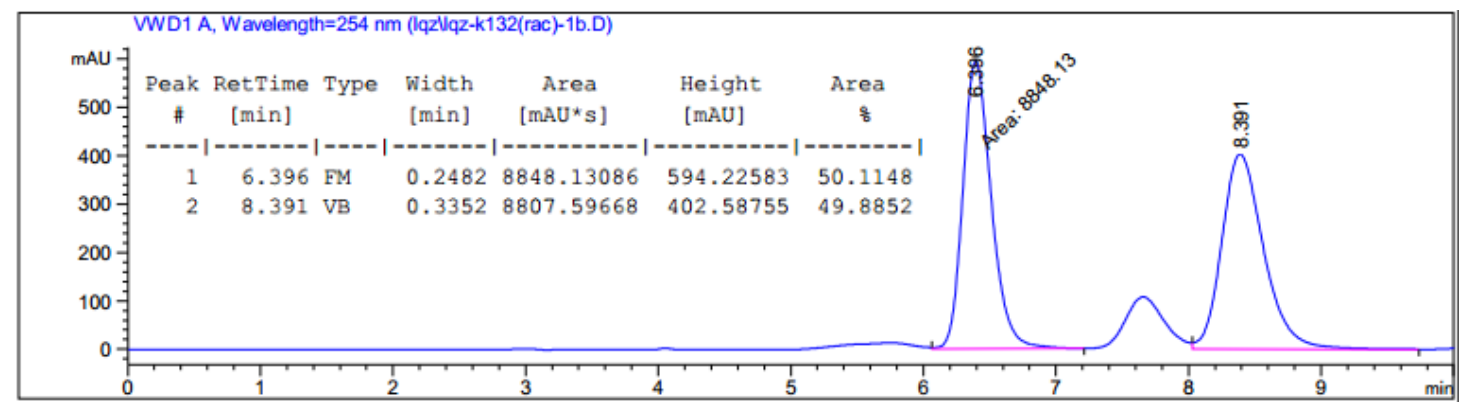

Chiral HPLC spectrum of $\mathbf{3 a i}$
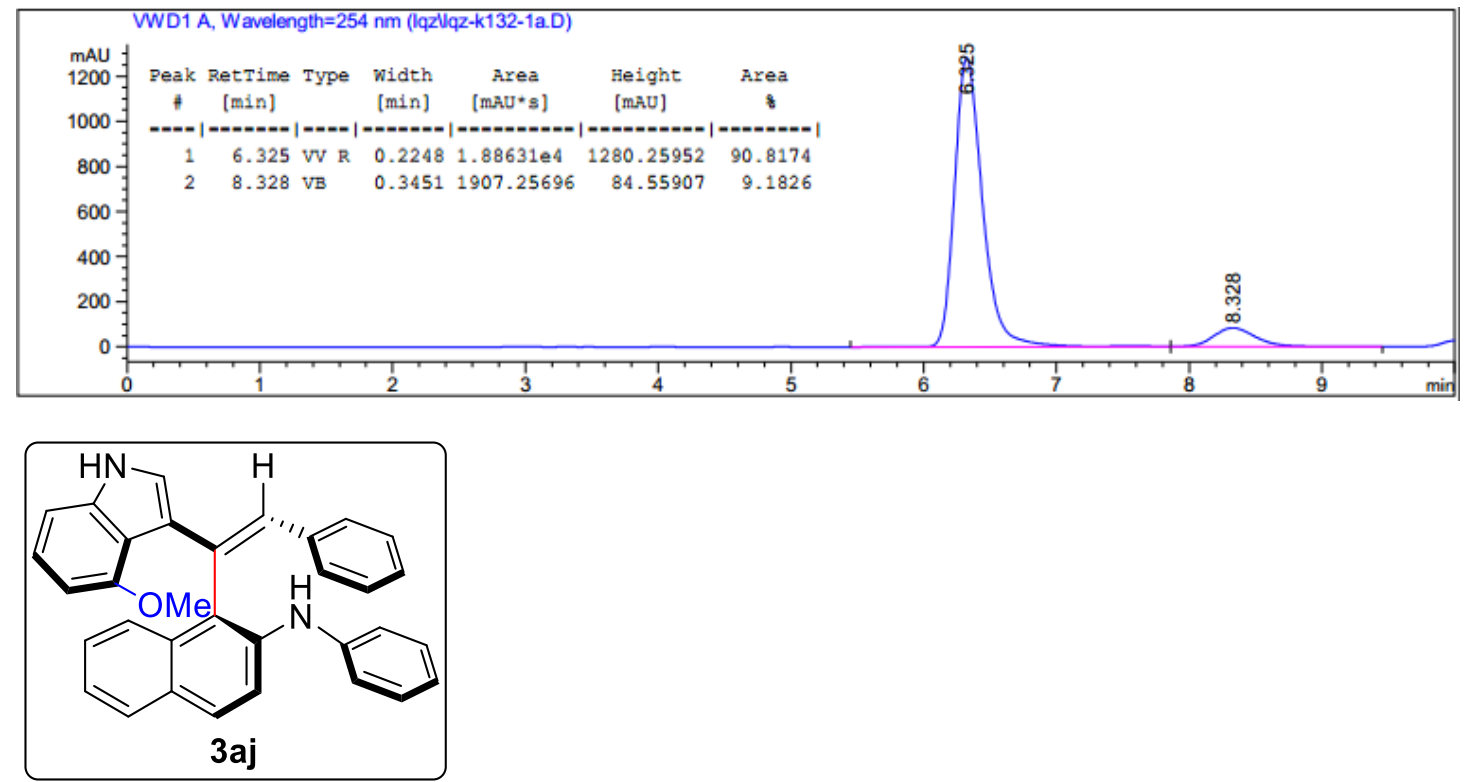

(aS)-(Z)-1-(1-(4-methoxy-1H-indol-3-yl)-2-phenylvinyl)-N-phenylnaphthalen-2-amine (3aj) According to procedure B, 3aj was obtained as a yellow foam, $42.4 \mathrm{mg}$ (91\% yield, $93 \%$ ee, Z: $\mathrm{E}=1.6: 1$ ). ${ }^{1}$ H NMR (400 MHz, CDCl $) \delta 8.02(\mathrm{~d}, J=8.1 \mathrm{~Hz}, 1 \mathrm{H}), 7.87(\mathrm{br}, 1 \mathrm{H}), 7.84-7.79(\mathrm{~m}, 2 \mathrm{H}), 7.72(\mathrm{~s}, 1 \mathrm{H})$, $7.63(\mathrm{~d}, J=8.9 \mathrm{~Hz}, 1 \mathrm{H}), 7.37-7.28(\mathrm{~m}, 2 \mathrm{H}), 7.20(\mathrm{td}, J=7.8,7.4,1.6 \mathrm{~Hz}, 3 \mathrm{H}), 7.10-7.02(\mathrm{~m}, 5 \mathrm{H})$, $6.94-6.89(\mathrm{~m}, 4 \mathrm{H}), 6.72(\mathrm{~d}, J=7.9 \mathrm{~Hz}, 1 \mathrm{H}), 6.66(\mathrm{~d}, J=2.7 \mathrm{~Hz}, 1 \mathrm{H}), 6.60(\mathrm{~s}, 1 \mathrm{H}), 4.05(\mathrm{~s}, 3 \mathrm{H})$.

${ }^{13}$ C NMR (101 MHz, $\left.\mathbf{C D C l}_{3}\right) \delta 154.4,143.3,138.5,137.7,137.5,133.7,133.1,129.3,129.1,129.0$, $128.23,128.15,128.0,127.9,126.7,126.5,125.4,124.3,124.1,123.4,123.2,121.2,120.0,118.9,118.0$, 115.5, 104.9, 101.1, 55.4.

HRMS (ESI) calculated for $[\mathrm{M}+\mathrm{H}]^{+} \mathrm{C}_{33} \mathrm{H}_{27} \mathrm{~N}_{2} \mathrm{O}^{+}, \mathrm{m} / \mathrm{z}$ : 467.2123, found: 467.2120.

M.P. $93-96^{\circ} \mathrm{C}$.

$[\alpha]_{20 \mathrm{D}}=-129^{\circ}\left(\mathrm{c}=0.2, \mathrm{CHCl}_{3}\right)$.

HPLC analysis: HPLC DAICEL CHIRALPAK AD-H, hexane/isopropyl alcohol = 90/10, flow rate = 1 $\mathrm{mL} / \mathrm{min}, \lambda=360 \mathrm{~nm}$ ), tR (major) $=9.0 \mathrm{~min}, \mathrm{tR}($ minor $)=9.9 \mathrm{~min}$, ee $=93 \%$.

Chiral HPLC spectrum of racemic $3 a j$ 


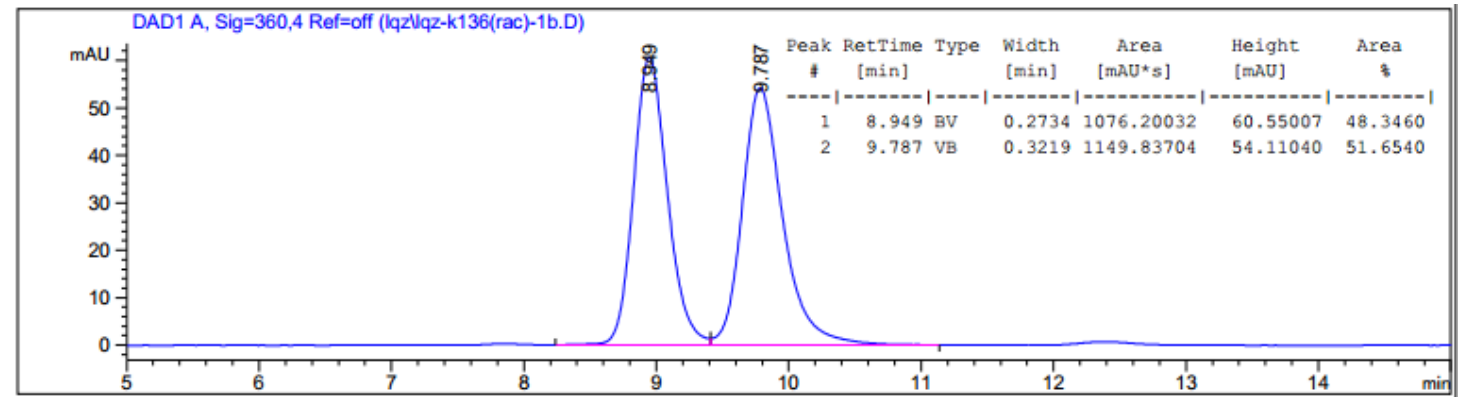

Chiral HPLC spectrum of $\mathbf{3 a j}$
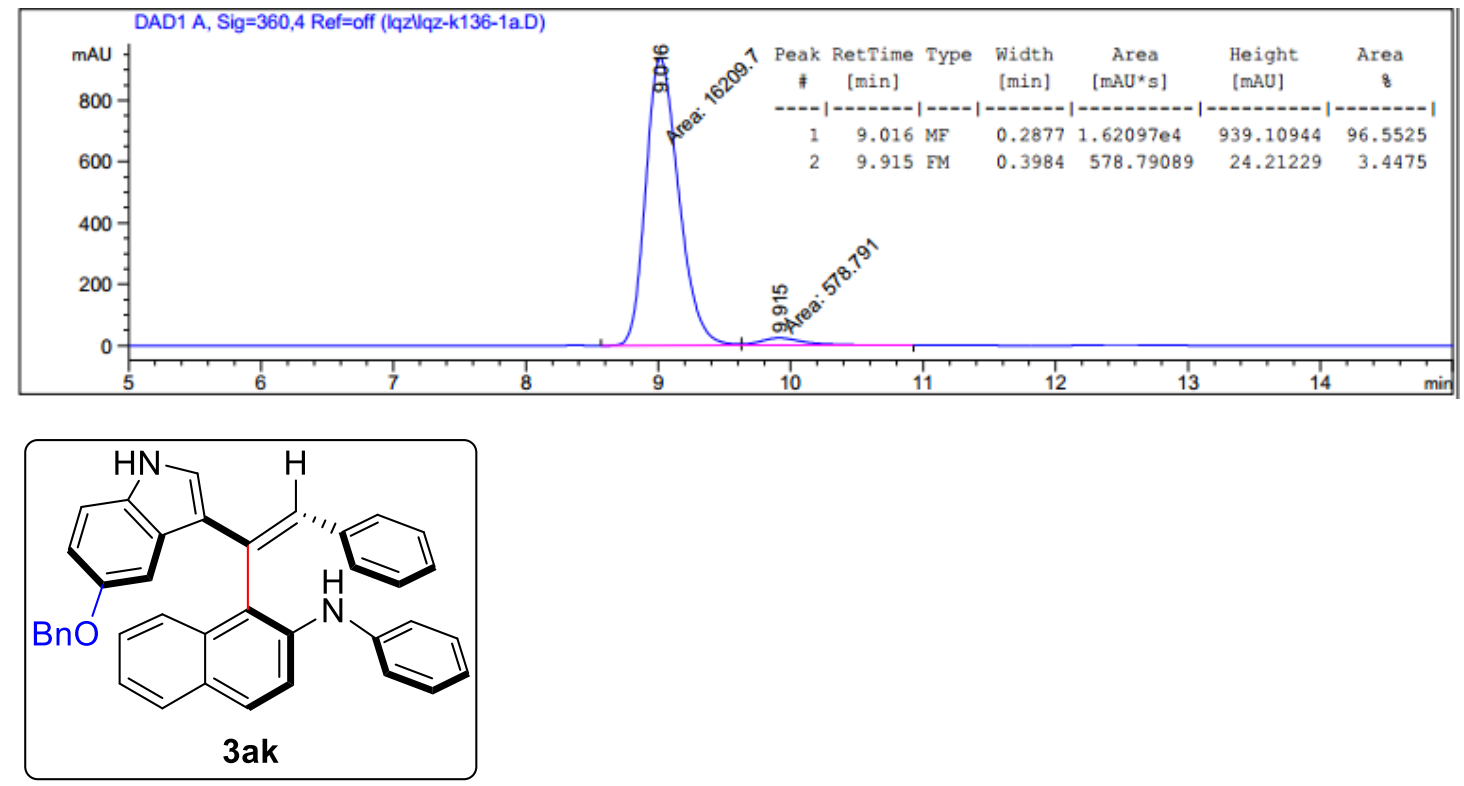

(aS)-(Z) -1-(1-(5-(benzyloxy)-1H-indol-3-yl)-2-phenylvinyl)-N-phenylnaphthalen-2-amine

(3ak)

According to procedure B, 3ak was obtained as a yellow oil, $32.0 \mathrm{mg}$ (59\% yield, 92\% ee, Z:E = 5:1).

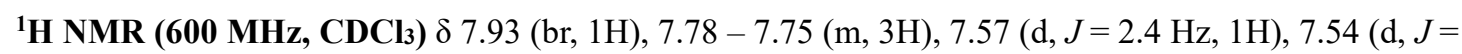
$9.0 \mathrm{~Hz}, 1 \mathrm{H}), 7.50(\mathrm{~d}, J=6.4 \mathrm{~Hz}, 3 \mathrm{H}), 7.40(\mathrm{t}, J=7.6 \mathrm{~Hz}, 2 \mathrm{H}), 7.35-7.32(\mathrm{~m}, 1 \mathrm{H}), 7.27-7.24(\mathrm{~m}, 4 \mathrm{H})$, $7.15-7.12(\mathrm{~m}, 2 \mathrm{H}), 7.03-6.96(\mathrm{~m}, 6 \mathrm{H}), 6.87-6.84(\mathrm{~m}, 3 \mathrm{H}), 6.74(\mathrm{~d}, J=2.7 \mathrm{~Hz}, 1 \mathrm{H}), 6.14(\mathrm{~s}, 1 \mathrm{H})$, $5.15-5.07(\mathrm{~m}, 2 \mathrm{H})$.

${ }^{13}$ C NMR (151 MHz, CDCl 3$) \delta 177.1,153.9,143.0,137.8,137.6,137.2,133.2,132.4,131.3,129.2$, $129.1,128.6,128.4,128.2,128.2,127.9,127.8,127.6,127.5,126.70,126.66,125.8,125.5,125.2,123.3$, $122.6,121.5,119.3,118.3,118.1,113.2,112.2,104.8,70.9$.

HRMS (ESI) calculated for $[\mathrm{M}+\mathrm{H}]^{+} \mathrm{C}_{39} \mathrm{H}_{31} \mathrm{~N}_{2} \mathrm{O}^{+}, \mathrm{m} / \mathrm{z}$ : 543.2436, found: 543.2431.

$[\alpha]_{20 \mathrm{D}}=-30^{\circ}\left(\mathrm{c}=0.2, \mathrm{CHCl}_{3}\right)$.

HPLC analysis: HPLC DAICEL CHIRALPAK AD-H, hexane/isopropyl alcohol = 60/40, flow rate $=1$ $\mathrm{mL} / \mathrm{min}, \lambda=365 \mathrm{~nm})$, tR (major) $=17.4 \mathrm{~min}, \mathrm{tR}($ minor $)=6.2 \mathrm{~min}$, ee $=92 \%$.

Chiral HPLC spectrum of racemic $3 a k$ 


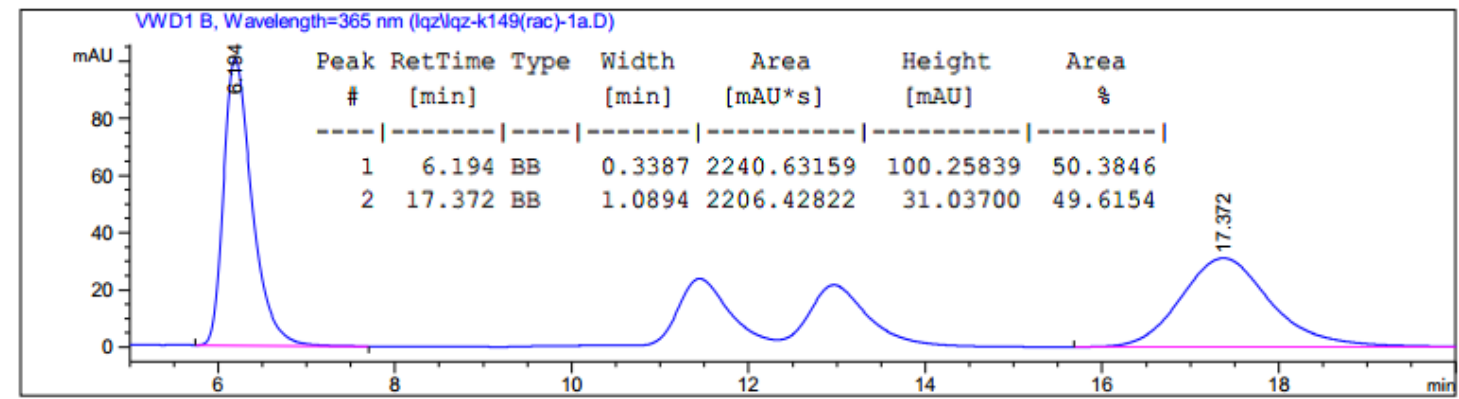

Chiral HPLC spectrum of $\mathbf{3 a k}$
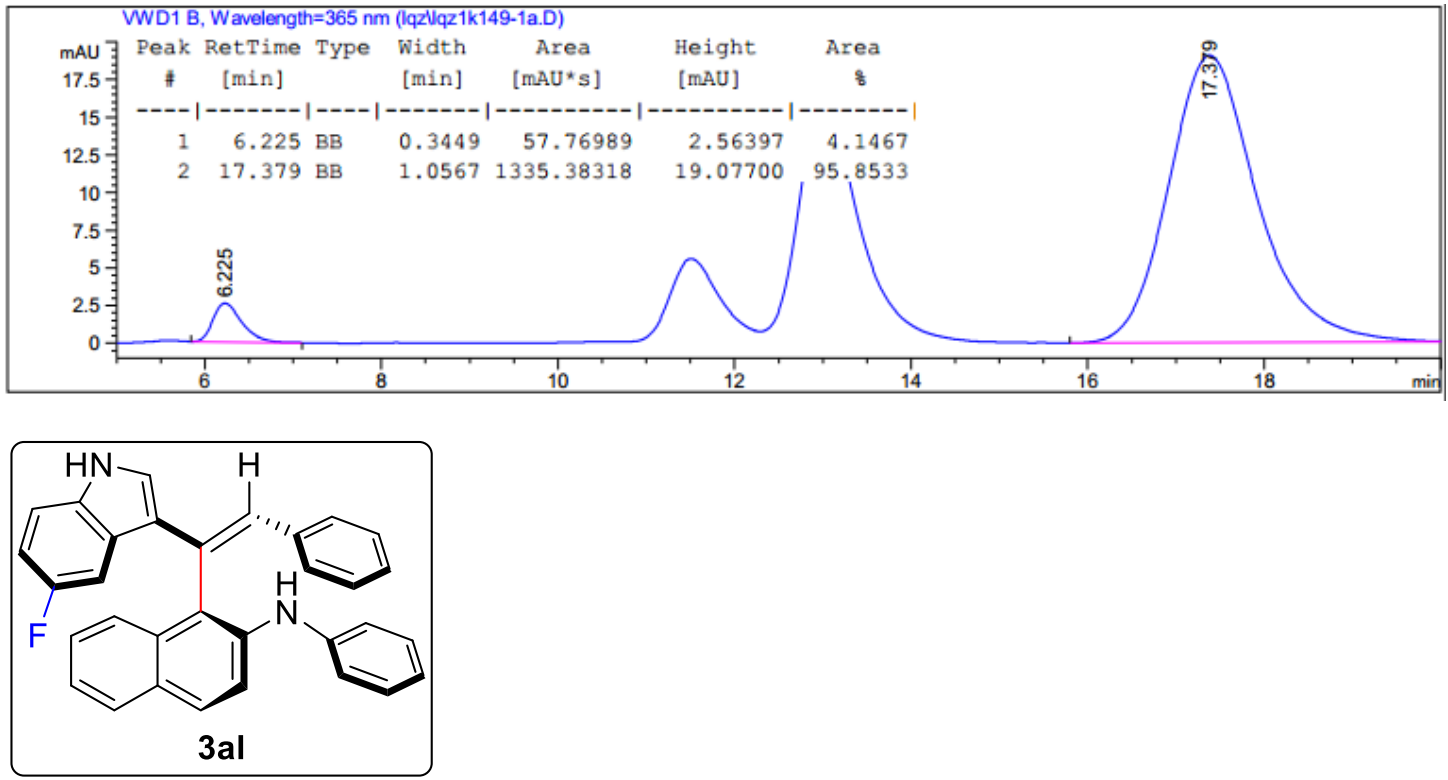

(aS)-(Z)-1-(1-(5-fluoro-1H-indol-3-yl)-2-phenylvinyl)-N-phenylnaphthalen-2-amine (3al)

According to procedure $\mathbf{B}$ under $5{ }^{\circ} \mathrm{C}, 3 \mathbf{a l}$ was obtained as a yellow foam, $40.0 \mathrm{mg}(88 \%$ yield, $90 \%$ ee, $\mathrm{Z}: \mathrm{E}=12: 1)$.

${ }^{1}$ H NMR (400 MHz, CDCl $) \delta 7.95(\mathrm{~s}, 1 \mathrm{H}), 7.81-7.77(\mathrm{~m}, 4 \mathrm{H}), 7.58(\mathrm{t}, J=4.5 \mathrm{~Hz}, 2 \mathrm{H}), 7.30-7.25$ (m, 2H), $7.24-7.22(\mathrm{~m}, 1 \mathrm{H}), 7.17(\mathrm{t}, J=7.9 \mathrm{~Hz}, 2 \mathrm{H}), 7.04-7.01(\mathrm{~m}, 6 \mathrm{H}), 6.91-6.87 \mathrm{~m}, 3 \mathrm{H}), 6.75(\mathrm{~d}$, $J=2.7 \mathrm{~Hz}, 1 \mathrm{H}), 6.16(\mathrm{~s}, 1 \mathrm{H})$.

${ }^{13} \mathbf{C}$ NMR (126 MHz, $\left.\mathbf{C D C l}_{3}\right) \delta 159.4,157.6,142.9,137.8,136.9,133.6,133.1,130.8,129.2,129.1$, 128.5, 128., 128.19, 127.99, 127.97, 126.9, 126.8, 125.4, 125.3, 125.0, 123.3, 122.3, 121.6, 119.2, 118.6, $118.6,118.1,112.24,112.16,111.0,110.8,106.0,105.8$.

HRMS (ESI) calculated for $[\mathrm{M}+\mathrm{H}]^{+} \mathrm{C}_{32} \mathrm{H}_{24} \mathrm{FN}_{2}{ }^{+}, \mathrm{m} / \mathrm{z}$ : 455.1924 , found: 455.1920 .

M.P. $83-85^{\circ} \mathrm{C}$.

$[\alpha]_{20} \mathrm{D}=-23.3^{\circ}\left(\mathrm{c}=0.3, \mathrm{CHCl}_{3}\right)$.

HPLC analysis: HPLC DAICEL CHIRALPAK AD-H, hexane/isopropyl alcohol $=90 / 10$, flow rate $=1$ $\mathrm{mL} / \mathrm{min}, \lambda=254 \mathrm{~nm}$ ), $\mathrm{tR}($ major $)=42.4 \mathrm{~min}, \mathrm{tR}(\operatorname{minor})=16.8 \mathrm{~min}, \mathrm{ee}=90 \%$.

Chiral HPLC spectrum of racemic 3 al 


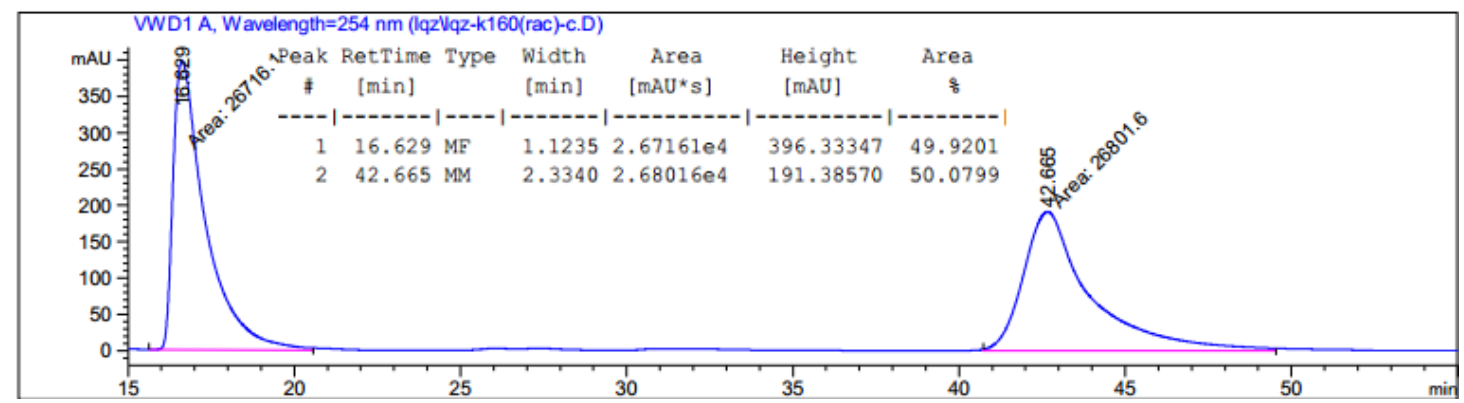

Chiral HPLC spectrum of $\mathbf{3 a l}$
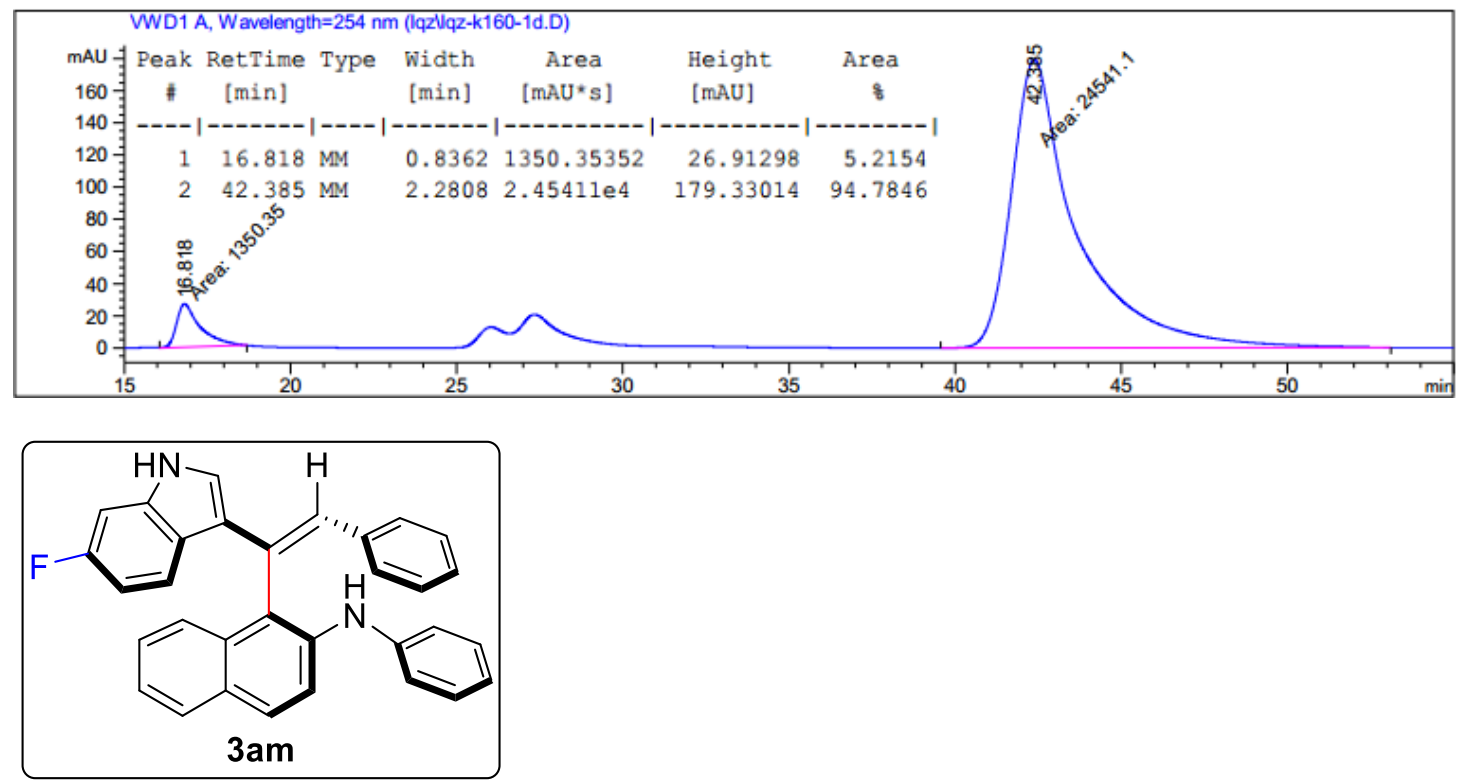

(aS)-(Z)-1-(1-(6-fluoro-1H-indol-3-yl)-2-phenylvinyl)-N-phenylnaphthalen-2-amine (3am)

According to procedure B, 3am was obtained as a yellow foam, $40.9 \mathrm{mg}$ (90\% yield, $90 \%$ ee, $\mathrm{Z}: \mathrm{E}=7: 1$ ). ${ }^{1}$ H NMR (400 MHz, CDCl $) \delta 8.06$ (dd, $\left.J=9.5,5.3 \mathrm{~Hz}, 1 \mathrm{H}\right), 7.98(\mathrm{~s}, 1 \mathrm{H}), 7.87-7.74(\mathrm{~m}, 3 \mathrm{H}), 7.65(\mathrm{~s}$, $1 \mathrm{H}), 7.58(\mathrm{~d}, J=9.0 \mathrm{~Hz}, 1 \mathrm{H}), 7.32-7.27(\mathrm{~m}, 2 \mathrm{H}), 7.18(\mathrm{t}, J=7.9 \mathrm{~Hz}, 2 \mathrm{H}), 7.06-7.02(\mathrm{~m}, 7 \mathrm{H}), 6.92-$ $6.88(\mathrm{~m}, 3 \mathrm{H}), 6.74(\mathrm{~d}, J=2.6 \mathrm{~Hz}, 1 \mathrm{H}), 6.17(\mathrm{~s}, 1 \mathrm{H})$.

${ }^{13} \mathbf{C}$ NMR (126 MHz, $\left.\mathbf{C D C l}_{3}\right) \delta 160.9,159.0,143.0,137.9,137.4,137.3,137.0,133.2,130.9,129.3$, $129.2,129.13,129.07,128.5,128.3,128.2,128.2,128.0,126.9,126.8,125.43,125.41,125.0,123.4$, 122.4, 121.8, 121.60, 121.58, 121.5, 119.3, 118.7, 118.2, 117.8, 109.4, 109.2, 98.1, 97.9.

HRMS (ESI) calculated for $[\mathrm{M}+\mathrm{H}]^{+} \mathrm{C}_{32} \mathrm{H}_{24} \mathrm{FN}_{2}{ }^{+}, \mathrm{m} / \mathrm{z}$ : 455.1924, found: 455.1922 .

M.P. $65-68^{\circ} \mathrm{C}$.

$[\alpha]_{20 \mathrm{D}}=-24.8^{\circ}\left(\mathrm{c}=0.5, \mathrm{CHCl}_{3}\right)$.

HPLC analysis: HPLC DAICEL CHIRALPAK AD-H, hexane/isopropyl alcohol $=70 / 30$, flow rate $=1$ $\mathrm{mL} / \mathrm{min}, \lambda=365 \mathrm{~nm}$ ), $\operatorname{tr}($ major $)=12.4 \mathrm{~min}, \operatorname{tr}(\operatorname{minor})=5.5 \mathrm{~min}$, ee $=90 \%$.

Chiral HPLC spectrum of racemic 3 am 


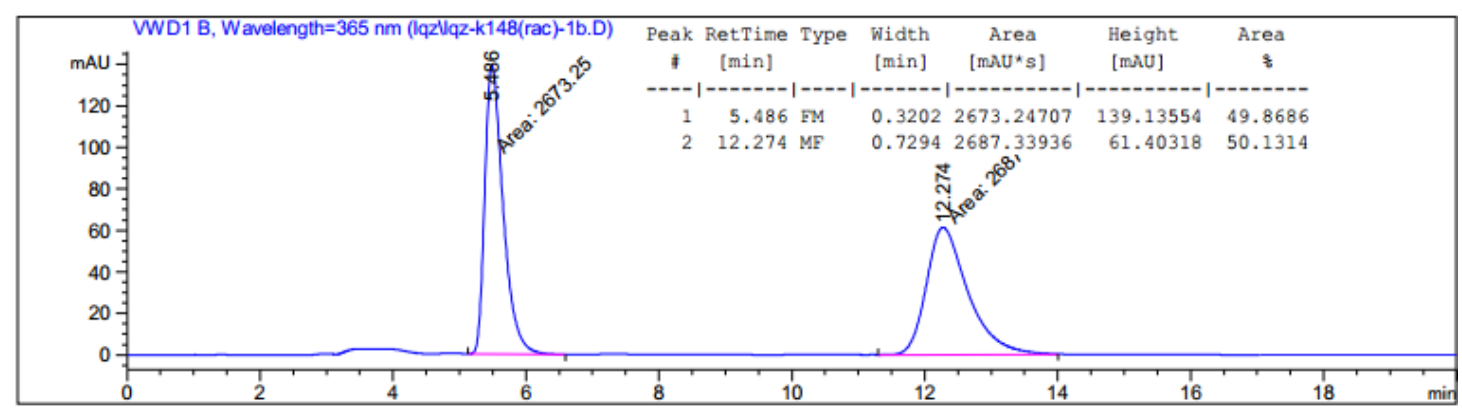

Chiral HPLC spectrum of $\mathbf{3 a m}$
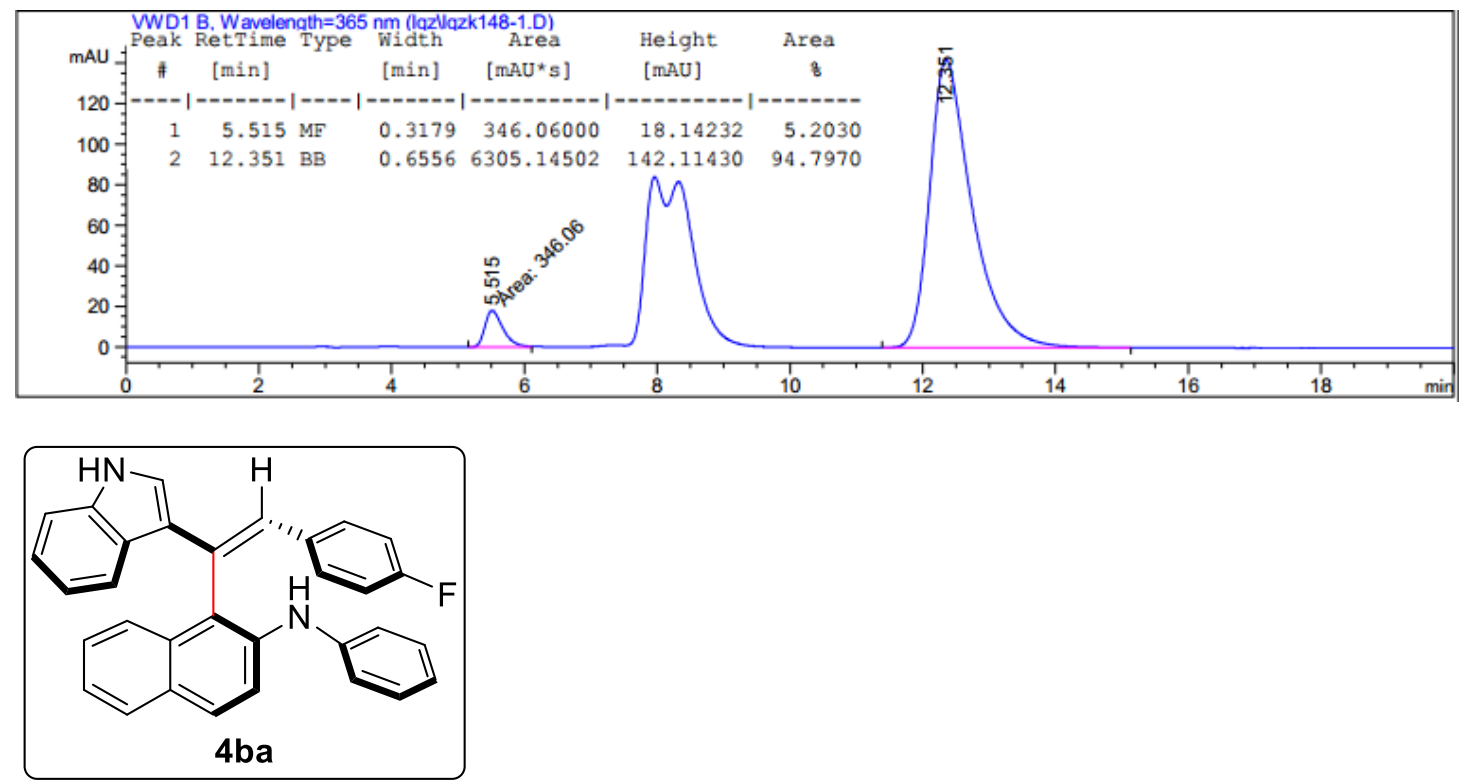

(aS)-(Z)-1-(2-(4-fluorophenyl)-1-(1H-indol-3-yl)vinyl)-N-phenylnaphthalen-2-amine (4ba)

According to procedure B, 4ba was obtained as a light yellow oil, $27.2 \mathrm{mg}(60 \%$ yield, $95 \%$ ee, Z:E = 10:1).

${ }^{1}$ H NMR (600 MHz, CDCl $) \delta 8.14(\mathrm{~d}, J=7.2 \mathrm{~Hz}, 1 \mathrm{H}), 7.91(\mathrm{~s}, 1 \mathrm{H}), 7.76(\mathrm{~d}, J=8.4 \mathrm{~Hz}, 2 \mathrm{H}), 7.73(\mathrm{~d}$, $J=8.0 \mathrm{~Hz}, 1 \mathrm{H}), 7.62(\mathrm{~s}, 1 \mathrm{H}), 7.54(\mathrm{~d}, J=9.0 \mathrm{~Hz}, 1 \mathrm{H}), 7.31-7.31(\mathrm{~m}, 1 \mathrm{H}), 7.27-7.22(\mathrm{~m}, 4 \mathrm{H}), 7.13(\mathrm{t}$, $J=7.9 \mathrm{~Hz}, 2 \mathrm{H}), 6.94-6.92(\mathrm{~m}, 2 \mathrm{H}), 6.847-6.83(\mathrm{~m}, 3 \mathrm{H}), 6.68(\mathrm{t}, J=8.8 \mathrm{~Hz}, 2 \mathrm{H}), 6.63(\mathrm{~d}, J=2.6 \mathrm{~Hz}$, $1 \mathrm{H}), 6.10(\mathrm{~s}, 1 \mathrm{H})$.

${ }^{13}$ C NMR (151 MHz, $\left.\mathbf{C D C l}_{3}\right) \delta 162.3,160.6,142.9,137.8,137.2,133.4,133.3,133.1,130.91,130.90$, $129.7,129.7,129.2,129.1,128.5,128.0,126.8,126.6,125.4,124.98,124.97,123.4,122.6,122.3,121.6$, $120.71,120.67,119.1,118.2,118.1,115.1,115.0,111.7$.

HRMS (ESI) calculated for $[\mathrm{M}+\mathrm{H}]^{+} \mathrm{C}_{32} \mathrm{H}_{24} \mathrm{FN}_{2}{ }^{+}, \mathrm{m} / \mathrm{z}$ : 455.1924 , found: 455.1922 . $[\alpha]^{20}{ }_{\mathrm{D}}=-19^{\circ}\left(\mathrm{c}=0.2, \mathrm{CHCl}_{3}\right)$.

HPLC analysis: HPLC DAICEL CHIRALPAK AD-H, hexane/isopropyl alcohol $=70 / 30$, flow rate $=1$ $\mathrm{mL} / \mathrm{min}, \lambda=365 \mathrm{~nm}$ ), $\mathrm{tR}($ major $)=8.1 \mathrm{~min}, \mathrm{tr}($ minor $)=4.8 \mathrm{~min}$, ee $=95 \%$.

Chiral HPLC spectrum of racemic $\mathbf{4 b a}$ 


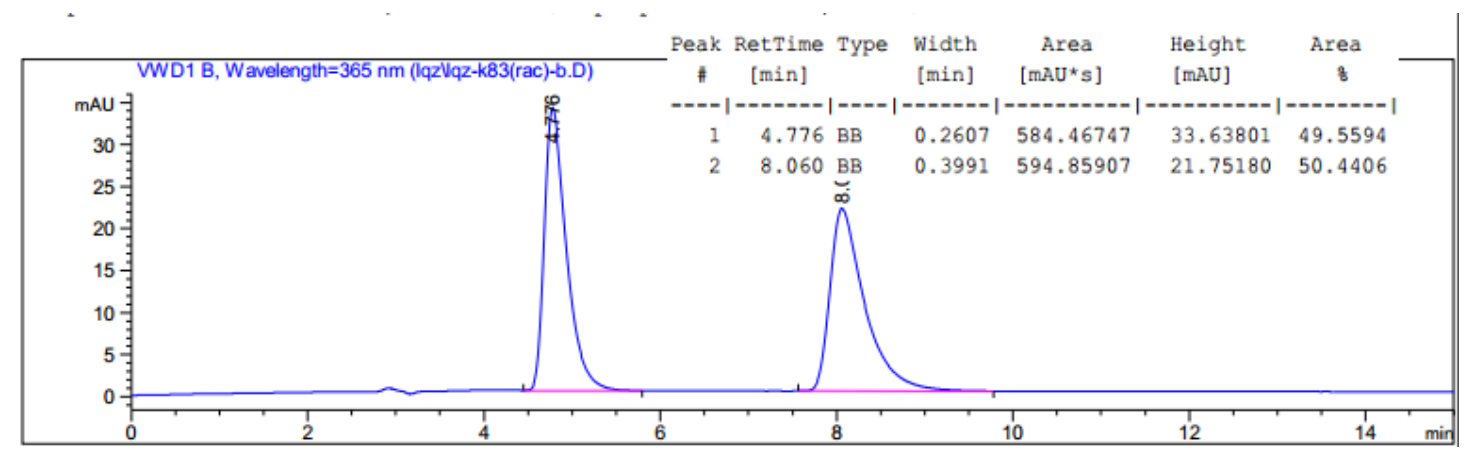

Chiral HPLC spectrum of $\mathbf{4 b a}$
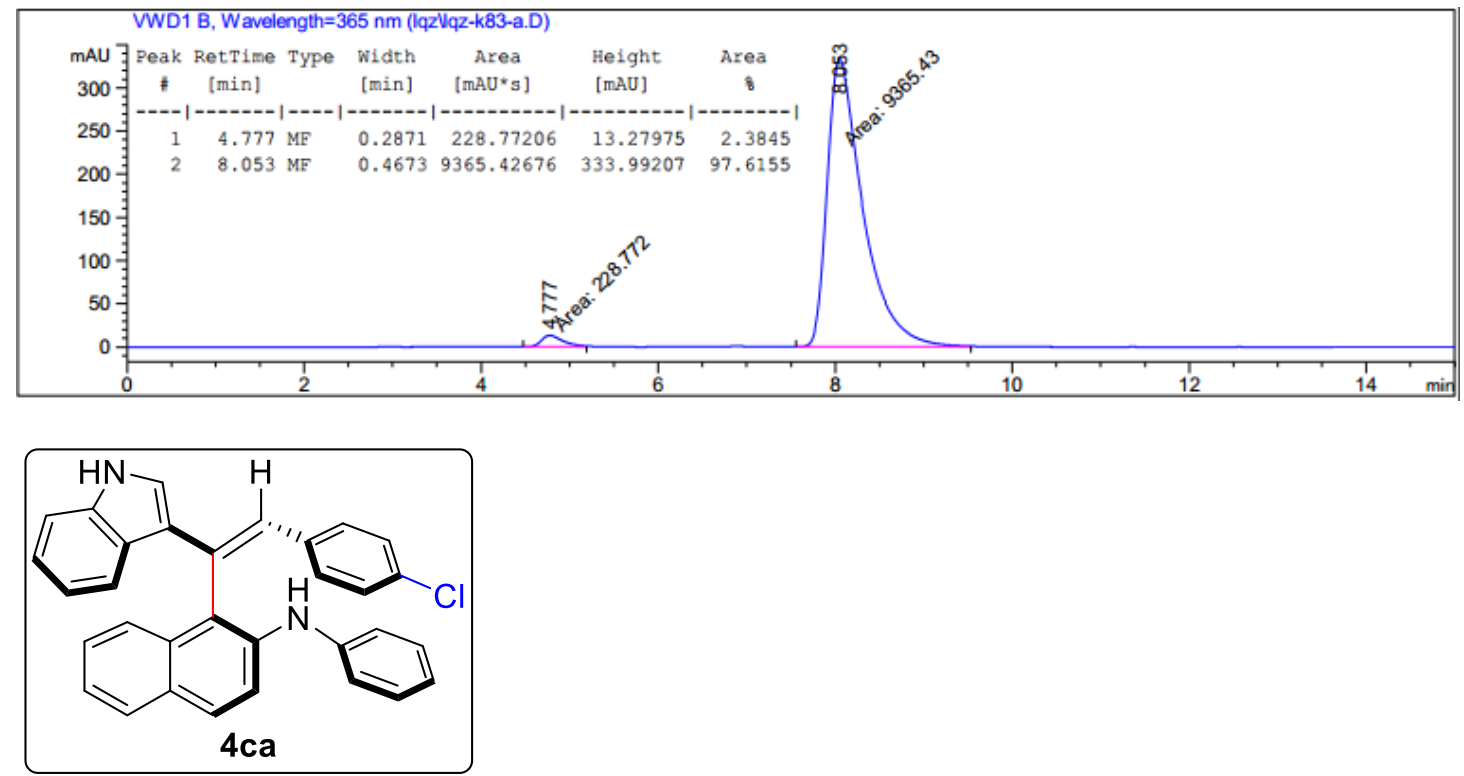

(aS)-(Z)-1-(2-(4-chlorophenyl)-1-(1H-indol-3-yl)vinyl)-N-phenylnaphthalen-2-amine (4ca)

According to procedure B, 4ca was obtained as a light yellow foam, $39.5 \mathrm{mg}$ ( $84 \%$ yield, $92 \%$ ee, Z:E = $11: 1)$.

${ }^{1}$ H NMR (500 MHz, CDCl $)$ ) $\delta 8.21-8.19(\mathrm{~m}, 1 \mathrm{H}), 8.05(\mathrm{~s}, 1 \mathrm{H}), 7.83-7.81(\mathrm{~m}, 2 \mathrm{H}), 7.76(\mathrm{dd}, J=7.6$, $1.8 \mathrm{~Hz}, 1 \mathrm{H}), 7.66(\mathrm{~s}, 1 \mathrm{H}), 7.59$ (d, $J=9.0 \mathrm{~Hz}, 1 \mathrm{H}), 7.41-7.39(\mathrm{~m}, 1 \mathrm{H}), 7.32-7.30(\mathrm{~m}, 4 \mathrm{H}), 7.21-7.17$ $(\mathrm{m}, 2 \mathrm{H}), 7.01-6.99(\mathrm{~m}, 2 \mathrm{H}), 6.95-6.92(\mathrm{~m}, 3 \mathrm{H}), 6.91-6.88(\mathrm{~m}, 2 \mathrm{H}), 6.74(\mathrm{~d}, J=2.7 \mathrm{~Hz}, 1 \mathrm{H}), 6.12(\mathrm{~s}$, $1 \mathrm{H})$.

${ }^{13}$ C NMR (126 MHz, CDCl $) \delta 142.9,137.9,137.3,135.8,133.1,132.127,132.0,129.4,129.3,129.2$, $128.6,128.3,128.0,126.9,126.5,125.7,125.0,125.0,123.5,122.8,122.2,121.7,120.9,120.8,119.113$, $118.3,118.2,111.8$.

HRMS (ESI) calculated for $[\mathrm{M}+\mathrm{H}]^{+} \mathrm{C}_{32} \mathrm{H}_{24} \mathrm{ClN}_{2}{ }^{+}, \mathrm{m} / \mathrm{z}: 471.1628$, found: 471.1689 .

M.P. $90-93{ }^{\circ} \mathrm{C}$.

$[\alpha]^{20}{ }_{D}=-22^{\circ}\left(\mathrm{c}=0.2, \mathrm{CHCl}_{3}\right)$.

HPLC analysis: HPLC DAICEL CHIRALPAK AD-H, hexane/isopropyl alcohol = 70/30, flow rate = 1 $\mathrm{mL} / \mathrm{min}, \lambda=365 \mathrm{~nm}$ ), tR (major) $=8.3 \mathrm{~min}, \mathrm{tR}($ minor $)=4.9 \mathrm{~min}$, ee $=92 \%$.

Chiral HPLC spectrum of racemic 4 ca 


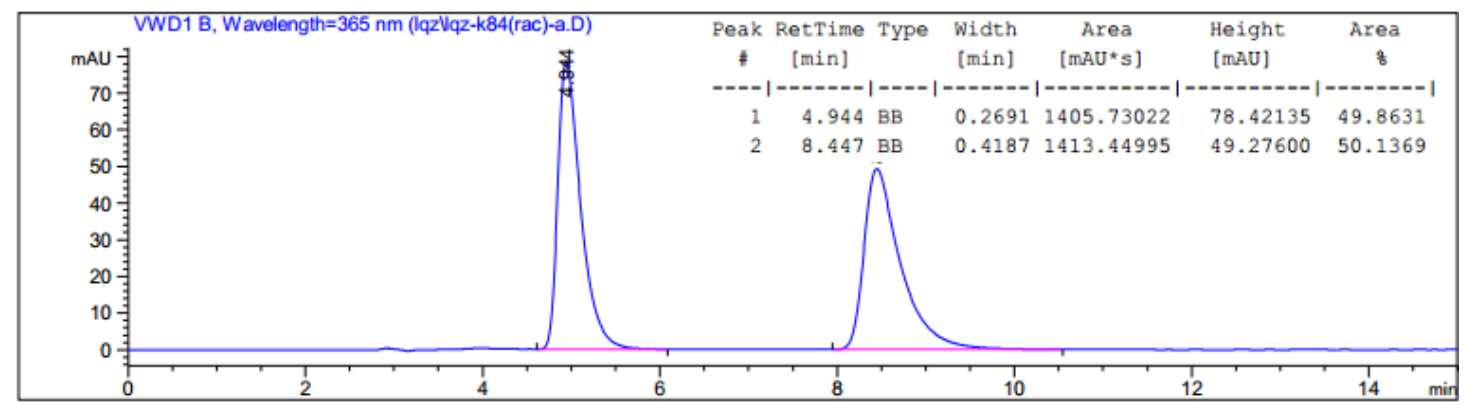

Chiral HPLC spectrum of $\mathbf{4 c a}$
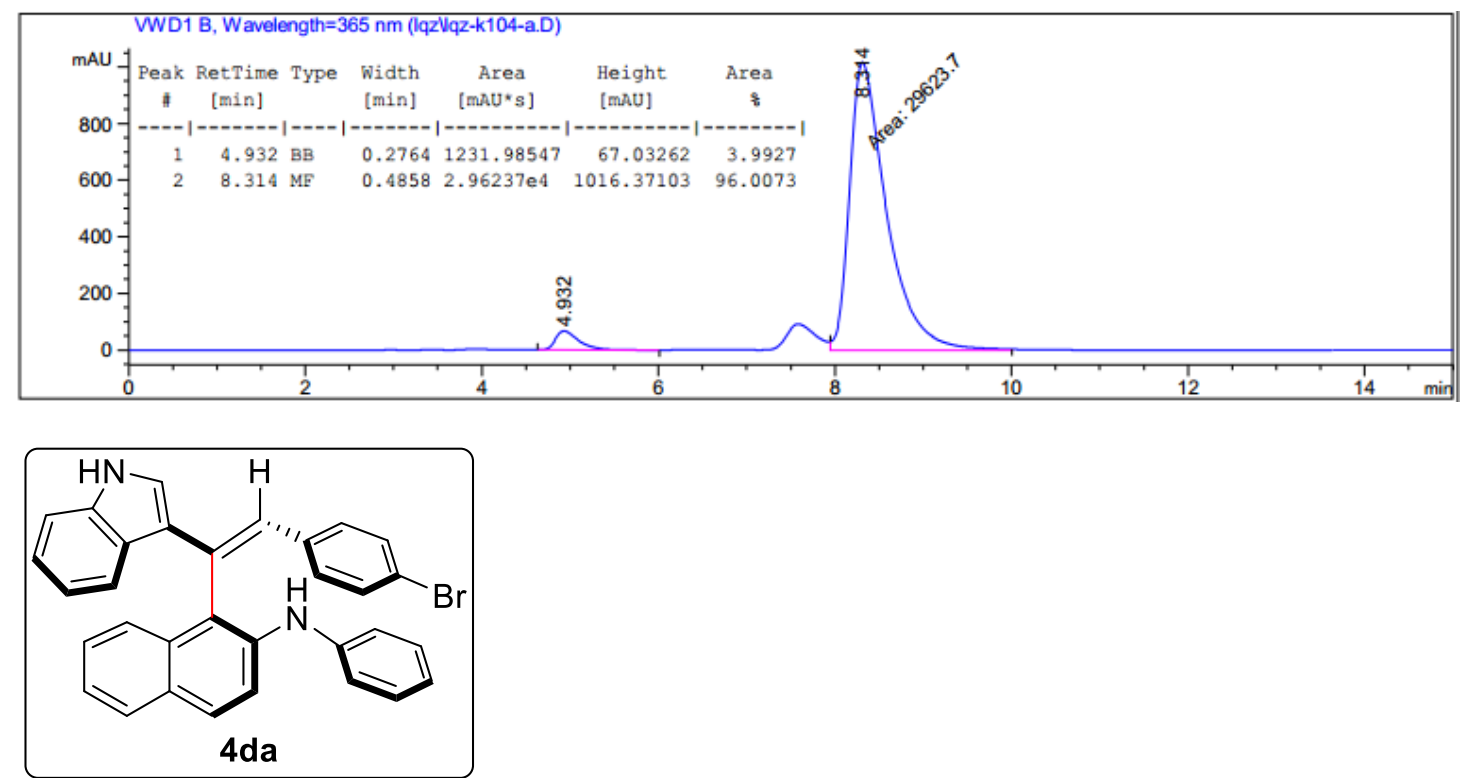

(aS)-(Z)-1-(2-(4-bromophenyl)-1-(1H-indol-3-yl)vinyl)-N-phenylnaphthalen-2-amine (4da)

According to procedure B, 4da was obtained as a brown foam, $46.3 \mathrm{mg}$ (90\% yield, 90\% ee, Z:E = 10:1). ${ }^{1}$ H NMR (500 MHz, CDCl $) \delta 8.18-8.16(\mathrm{~m}, 1 \mathrm{H}), 8.07(\mathrm{~s}, 1 \mathrm{H}), 7.81-7.78(\mathrm{~m}, 2 \mathrm{H}), 7.73(\mathrm{~d}, J=8.5$ $\mathrm{Hz}, 2 \mathrm{H}), 7.61(\mathrm{~s}, 1 \mathrm{H}), 7.56(\mathrm{~d}, J=9.0 \mathrm{~Hz}, 1 \mathrm{H}), 7.38-7.36(\mathrm{~m}, 1 \mathrm{H}), 7.29-7.27(\mathrm{~m}, 4 \mathrm{H}), 7.17(\mathrm{t}, J=7.9$ $\mathrm{Hz}, 2 \mathrm{H}), 7.13(\mathrm{~d}, J=8.6 \mathrm{~Hz}, 2 \mathrm{H}), 6.90(\mathrm{t}, J=7.4 \mathrm{~Hz}, 2 \mathrm{H}), 6.87-6.84(\mathrm{~m}, 4 \mathrm{H}), 6.72-6.70(\mathrm{~m}, 1 \mathrm{H}), 6.09$ $(\mathrm{s}, 1 \mathrm{H})$.

${ }^{13}$ C NMR (126 MHz, $\left.\mathbf{C D C l}_{3}\right) \delta 142.9,137.8,137.3,136.2,133.1,132.1,131.2,129.7,129.2,129.1$, $128.6,128.0,126.8,126.4,125.7,124.93,124.91,123.4,122.7,122.2,121.6,120.8,120.7,120.3,119.1$, $118.3,118.1,111.8$.

HRMS (ESI) calculated for $[\mathrm{M}+\mathrm{H}]^{+} \mathrm{C}_{32} \mathrm{H}_{24} \mathrm{BrN}_{2}{ }^{+}, \mathrm{m} / \mathrm{z}: 515.1123$, found: 515.1100 .

M.P. $85-88^{\circ} \mathrm{C}$.

$[\alpha]^{20}{ }_{\mathrm{D}}=-23^{\circ}\left(\mathrm{c}=0.2, \mathrm{CHCl}_{3}\right)$.

HPLC analysis: HPLC DAICEL CHIRALPAK AD-H, hexane/isopropyl alcohol = 70/30, flow rate $=1$ $\mathrm{mL} / \mathrm{min}, \lambda=365 \mathrm{~nm})$, tR $($ major $)=8.9 \mathrm{~min}, \mathrm{tR}($ minor $)=5.2 \mathrm{~min}$, ee $=90 \%$.

Chiral HPLC spectrum of racemic 4 da 


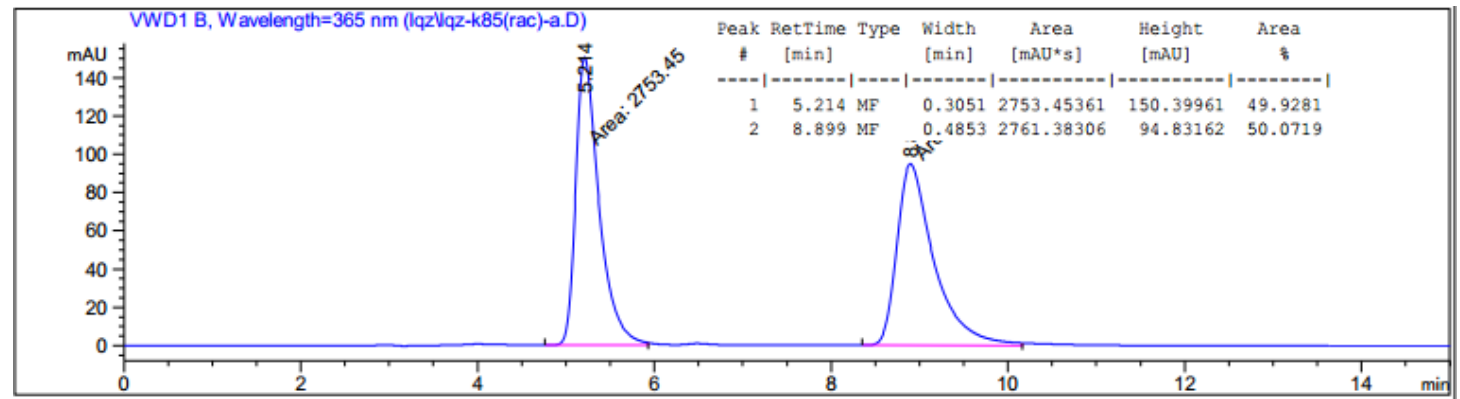

Chiral HPLC spectrum of $\mathbf{4 d a}$
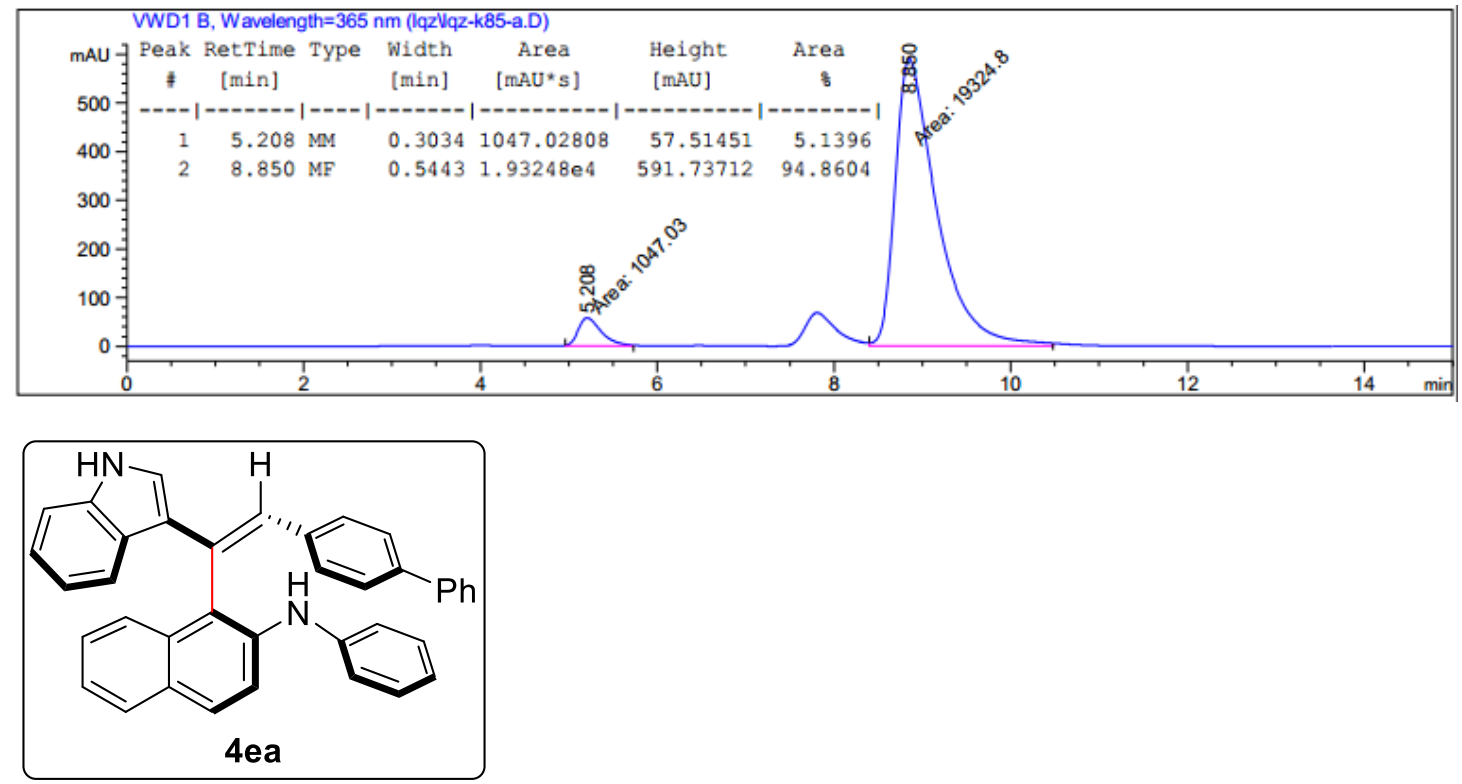

(aS)-(Z)-1-(2-([1,1'-biphenyl]-4-yl)-1-(1H-indol-3-yl)vinyl)-N-phenylnaphthalen-2-amine (4ea)

According to procedure B, 4ea was obtained as a yellow oil, $44.0 \mathrm{mg}$ (86\% yield, 90\% ee, Z:E = 9:1).

${ }^{1}$ H NMR (400 MHz, CDCl $) \delta 8.28-8.26(\mathrm{~m}, 1 \mathrm{H}), 7.96$ (s, 1H), $7.87-7.84(\mathrm{~m}, 3 \mathrm{H}), 7.78(\mathrm{~s}, 1 \mathrm{H}), 7.64$ $(\mathrm{d}, J=9.0 \mathrm{~Hz}, 1 \mathrm{H}), 7.53-7.50(\mathrm{~m}, 2 \mathrm{H}), 7.42-7.36(\mathrm{~m}, 3 \mathrm{H}), 7.35-7.30(\mathrm{~m}, 7 \mathrm{H}), 7.20-7.16(\mathrm{~m}, 2 \mathrm{H})$, $7.13-7.10(\mathrm{~m}, 2 \mathrm{H}), 6.93-6.89(\mathrm{~m}, 3 \mathrm{H}), 6.23(\mathrm{~s}, 1 \mathrm{H})$.

${ }^{13}$ C NMR (126 MHz, CDCl 3$) \delta 143.1,140.7,139.2,137.9,137.3,136.4,133.3,131.4,129.4,129.2$, $128.7,128.5,128.0,127.5,127.1,126.9,126.8,125.5,125.2,125.1,123.5,122.8,122.7,121.6,120.9$, $120.8,119.2,118.5,118.4,111.8$.

HRMS (ESI) calculated for $[\mathrm{M}+\mathrm{H}]^{+} \mathrm{C}_{38} \mathrm{H}_{29} \mathrm{~N}_{2}{ }^{+}, \mathrm{m} / \mathrm{z}: 513.2331$, found: 513.2326.

$[\alpha]^{20}{ }_{\mathrm{D}}=-66^{\circ}\left(\mathrm{c}=0.2, \mathrm{CHCl}_{3}\right)$.

HPLC analysis: HPLC DAICEL CHIRALPAK AD-H, hexane/isopropyl alcohol = 70/30, flow rate = 1 $\mathrm{mL} / \mathrm{min}, \lambda=254 \mathrm{~nm}$ ), tR (major) $=9.0 \mathrm{~min}, \mathrm{tR}($ minor $)=6.3 \mathrm{~min}$, ee $=90 \%$.

Chiral HPLC spectrum of racemic $\mathbf{4 e a}$ 


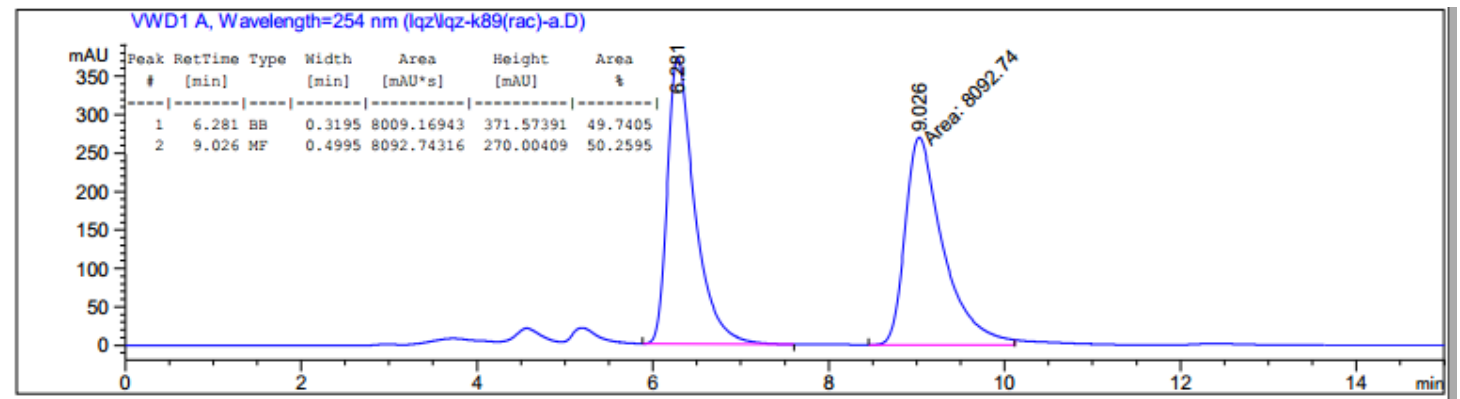

Chiral HPLC spectrum of $\mathbf{4 e a}$
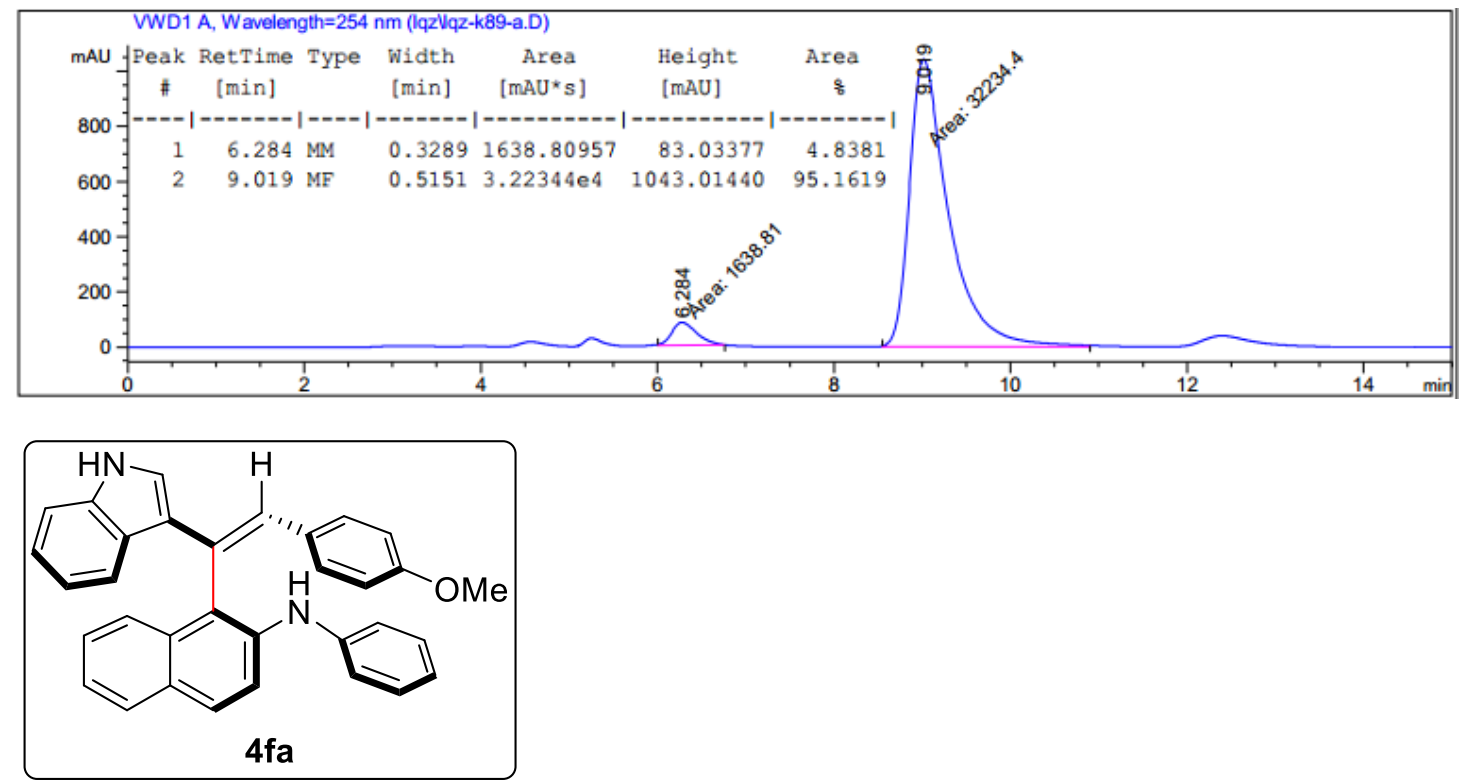

(aS)-(Z)-1-(1-(1H-indol-3-yl)-2-(4-methoxyphenyl)vinyl)-N-phenylnaphthalen-2-amine (4fa) According to procedure $\mathbf{B}, \mathbf{4 f a}$ was obtained as a yellow foam, $28.9 \mathrm{mg}(62 \%$ yield, $90 \%$ ee, $\mathrm{Z}: \mathrm{E}=12: 1)$. ${ }^{1}$ H NMR (400 MHz, CDCl $) \delta 8.22-8.20$ (m, 1H), 7.98 (s, 1H), $7.83-7.80(\mathrm{~m}, 3 \mathrm{H}), 7.67$ (s, 1H), 7.62 $(\mathrm{d}, J=8.9 \mathrm{~Hz}, 1 \mathrm{H}), 7.39-7.37(\mathrm{~m}, 1 \mathrm{H}), 7.32-7.28(\mathrm{~m}, 4 \mathrm{H}), 7.20-7.16(\mathrm{~m}, 2 \mathrm{H}), 6.98-6.95(\mathrm{~m}, 2 \mathrm{H})$, $6.93-6.88(\mathrm{~m}, 3 \mathrm{H}), 6.71(\mathrm{~d}, J=2.7 \mathrm{~Hz}, 1 \mathrm{H}), 6.62-6.60(\mathrm{~m}, 2 \mathrm{H}), 6.23(\mathrm{~s}, 1 \mathrm{H}), 3.70(\mathrm{~s}, 3 \mathrm{H})$.

${ }^{13}$ C NMR (101 MHz, $\left.\mathbf{C D C l}_{3}\right) \delta 158.4,143.1,137.8,137.3,133.2,130.0,129.5,129.3,129.1,129.0$, $128.3,127.9,127.6,126.7,125.2,125.2,124.9,123.3,122.9,122.5,121.42,120.8,120.6,119.1,118.7$, 118.2, 113.7, 111.7, 55.1.

HRMS (ESI) calculated for $[\mathrm{M}+\mathrm{H}]^{+} \mathrm{C}_{33} \mathrm{H}_{27} \mathrm{~N}_{2} \mathrm{O}^{+}$, m/z: 467.2123, found: 467.2119.

M.P. $85-87^{\circ} \mathrm{C}$.

$[\alpha]^{20}{ }_{\mathrm{D}}=-70^{\circ}\left(\mathrm{c}=0.2, \mathrm{CHCl}_{3}\right)$.

HPLC analysis: HPLC DAICEL CHIRALPAK AD-H, hexane/isopropyl alcohol = 70/30, flow rate = 1 $\mathrm{mL} / \mathrm{min}, \lambda=254 \mathrm{~nm})$, tR (major) $=14.8 \mathrm{~min}, \mathrm{tR}($ minor $)=6.6 \mathrm{~min}$, ee $=90 \%$.

Chiral HPLC spectrum of racemic $\mathbf{4 f a}$ 


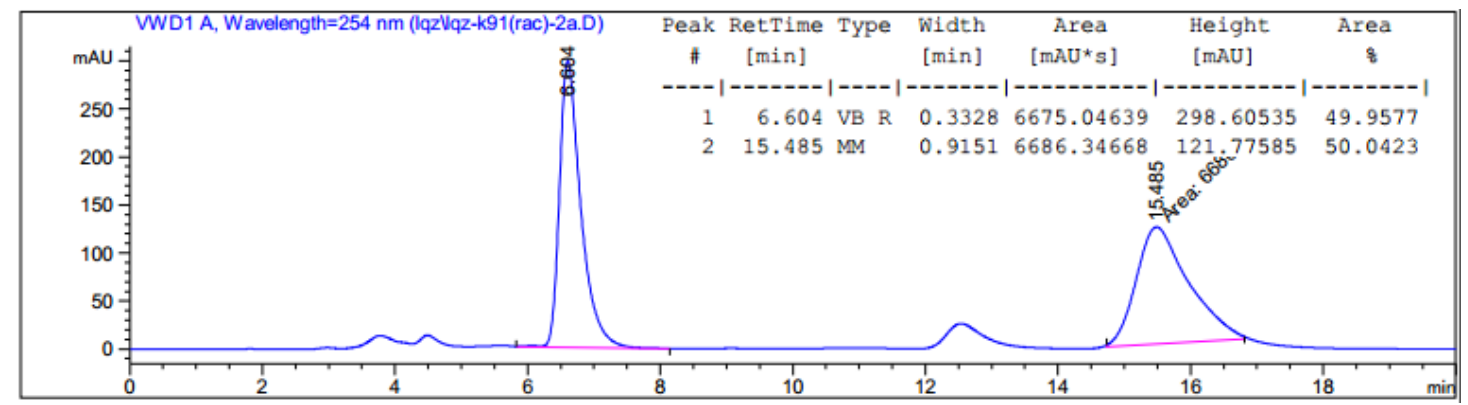

Chiral HPLC spectrum of $\mathbf{4 f a}$
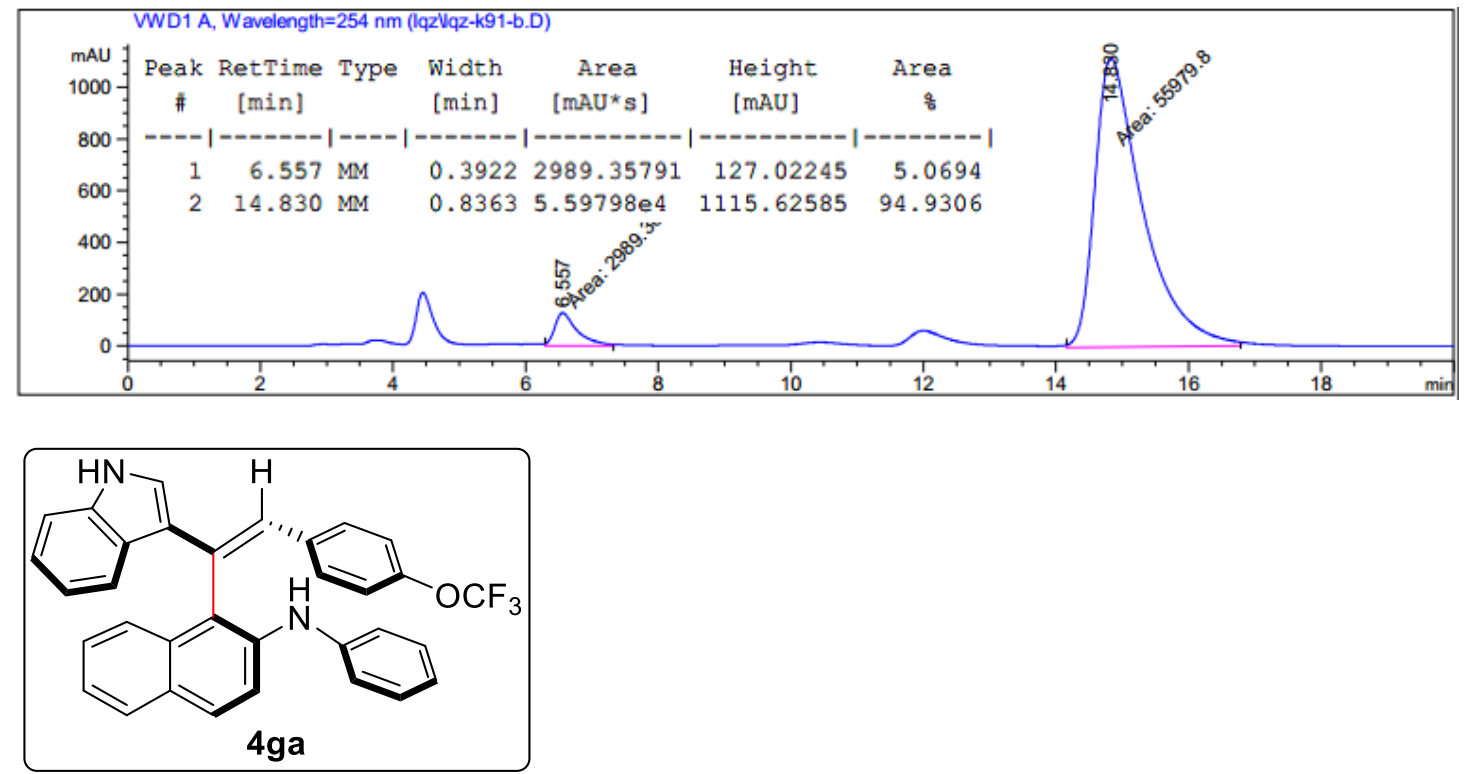

(aS)-(Z)-1-(1-(1H-indol-3-yl)-2-(4-(trifluoromethoxy)phenyl)vinyl)-N-phenylnaphthalen-2amine (4ga)

According to procedure $\mathbf{B}$, but the solvent is $\mathrm{CH}_{2} \mathrm{Cl}_{2}, 4$ ga was obtained as a yellow oil, $35.4 \mathrm{mg}(68 \%$ yield, $90 \%$ ee, $\mathrm{Z}: \mathrm{E}=10: 1)$.

${ }^{1} \mathbf{H}$ NMR (500 MHz, CDCl 3$) \delta 8.22-8.20(\mathrm{~m}, 1 \mathrm{H}), 8.04(\mathrm{~s}, 1 \mathrm{H}), 7.84(\mathrm{~d}, J=2.7 \mathrm{~Hz}, 2 \mathrm{H}), 7.78-7.77$ (m, 1H), $7.69(\mathrm{~s}, 1 \mathrm{H}), 7.58(\mathrm{dd}, J=8.9,1.1 \mathrm{~Hz}, 1 \mathrm{H}), 7.41-7.40(\mathrm{~m}, 1 \mathrm{H}), 7.34-7.30$ (m, 4H), 7.18 (dd, $J=8.3,7.1 \mathrm{~Hz}, 2 \mathrm{H}), 7.02(\mathrm{~d}, J=8.8 \mathrm{~Hz}, 2 \mathrm{H}), 6.94-6.86(\mathrm{~m}, 5 \mathrm{H}), 6.74(\mathrm{~d}, J=2.7 \mathrm{~Hz}, 1 \mathrm{H}), 6.12(\mathrm{~s}, 1 \mathrm{H})$. ${ }^{13}$ C NMR (126 MHz, $\left.\mathbf{C D C l}_{3}\right) \delta$ 147.6, 142.9, 138.0, 137.3, 136.0, 133.2, 132.2, 129.4, 129.3, 129.2, $128.7,128.1,126.9,126.2,125.7,124.99,124.96,123.5,122.8,122.0,121.8,121.4,120.9,120.7,120.6$, $119.4,119.3,118.3,118.1,111.8$.

HRMS (ESI) calculated for $[\mathrm{M}+\mathrm{H}]^{+} \mathrm{C}_{33} \mathrm{H}_{24} \mathrm{~F}_{3} \mathrm{~N}_{2} \mathrm{O}^{+}, \mathrm{m} / \mathrm{z}: 521.1841$, found: 521.1801. $[\alpha]^{20}{ }_{\mathrm{D}}=3^{\circ}\left(\mathrm{c}=0.2, \mathrm{CHCl}_{3}\right)$.

HPLC analysis: HPLC DAICEL CHIRALPAK AD-H, hexane/isopropyl alcohol = 70/30, flow rate = 1 $\mathrm{mL} / \mathrm{min}, \lambda=254 \mathrm{~nm}$ ), $\mathrm{tR}$ (major) $=5.8 \mathrm{~min}, \mathrm{tR}($ minor $)=4.0 \mathrm{~min}$, ee $=90 \%$.

Chiral HPLC spectrum of racemic $\mathbf{4 g a}$ 


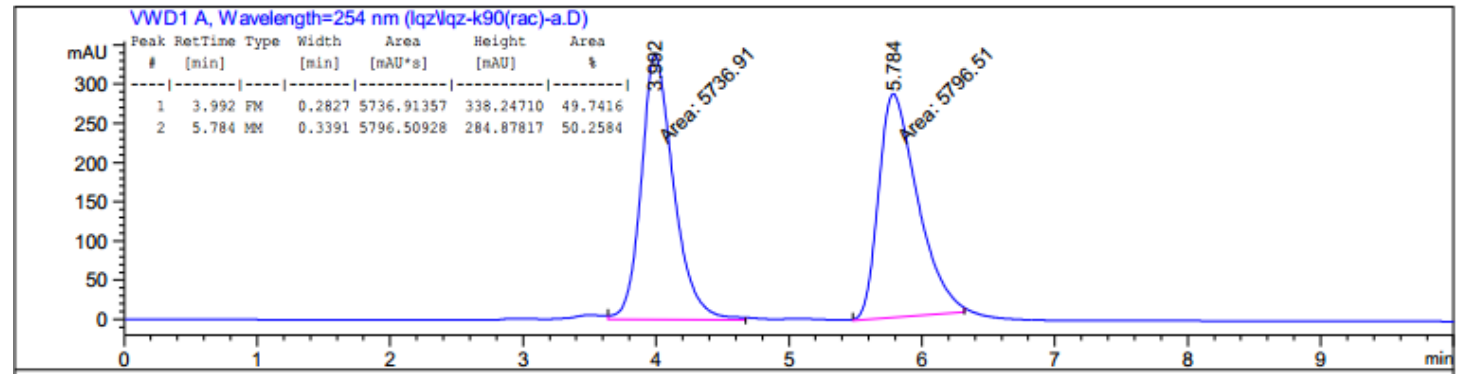

Chiral HPLC spectrum of $\mathbf{4 g a}$
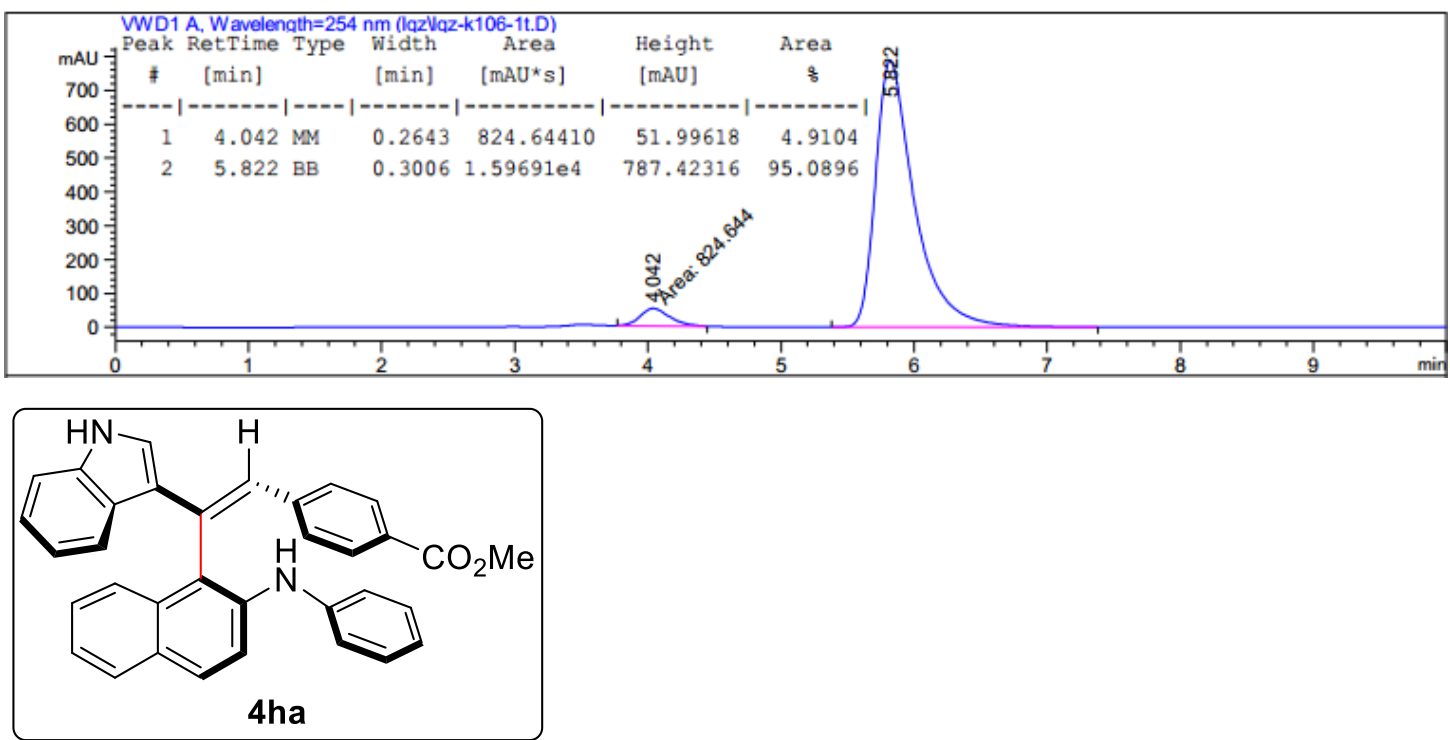

(aS)-methyl (Z)-4-(2-(1H-indol-3-yl)-2-(2-(phenylamino)naphthalen-1-yl)vinyl)benzoate (4ha)

According to procedure B, tha was obtained as a yellow solid, $42.0 \mathrm{mg}(85 \%$ yield, $90 \%$ ee, $\mathrm{Z}: \mathrm{E}=10: 1)$. ${ }^{1}$ H NMR (400 MHz, CDCl $)$ ) 8.24 - $8.214(\mathrm{~m}, 1 \mathrm{H}), 8.13(\mathrm{~s}, 1 \mathrm{H}), 7.84-7.81(\mathrm{~m}, 2 \mathrm{H}), 7.77$ - 7.70 (m, 4H), 7.59 (d, $J=8.9 \mathrm{~Hz}, 1 \mathrm{H}), 7.42-7.39(\mathrm{~m}, 1 \mathrm{H}), 7.34-7.28(\mathrm{~m}, 4 \mathrm{H}), 7.20-7.15$ (m, 2H), 7.07 (d, $J$ $=8.4 \mathrm{~Hz}, 2 \mathrm{H}), 6.93-6.87(\mathrm{~m}, 3 \mathrm{H}), 6.77(\mathrm{~d}, J=2.7 \mathrm{~Hz}, 1 \mathrm{H}), 6.10(\mathrm{~s}, 1 \mathrm{H}), 3.84(\mathrm{~s}, 3 \mathrm{H})$.

${ }^{13}$ C NMR (101 MHz, $\left.\mathbf{C D C l}_{3}\right) \delta 167.0,142.8,142.0,137.9,137.4,134.0,133.0,129.5,129.3,129.2$, $128.7,128.04,128.00,127.7,126.9,126.6,126.2,125.0,124.9,123.5,122.9,122.1,121.7,121.0,120.8$, $119.2,118.2,118.1,111.9,51.9$.

HRMS (ESI) calculated for $[\mathrm{M}+\mathrm{H}]^{+} \mathrm{C}_{34} \mathrm{H}_{27} \mathrm{~N}_{2} \mathrm{O}_{2}{ }^{+}, \mathrm{m} / \mathrm{z}$ : 495.2073, found: 495.2070.

M.P. $79-81^{\circ} \mathrm{C}$.

$[\alpha]^{20}{ }_{\mathrm{D}}=-52^{\circ}\left(\mathrm{c}=0.2, \mathrm{CHCl}_{3}\right)$.

HPLC analysis: HPLC DAICEL CHIRALPAK AD-H, hexane/isopropyl alcohol = 70/30, flow rate $=1$ $\mathrm{mL} / \mathrm{min}, \lambda=365 \mathrm{~nm}$ ), $\mathrm{tR}$ (major) $=8.4 \mathrm{~min}, \mathrm{tR}($ minor $)=7.3 \mathrm{~min}$, ee $=90 \%$.

Chiral HPLC spectrum of racemic 4 ha

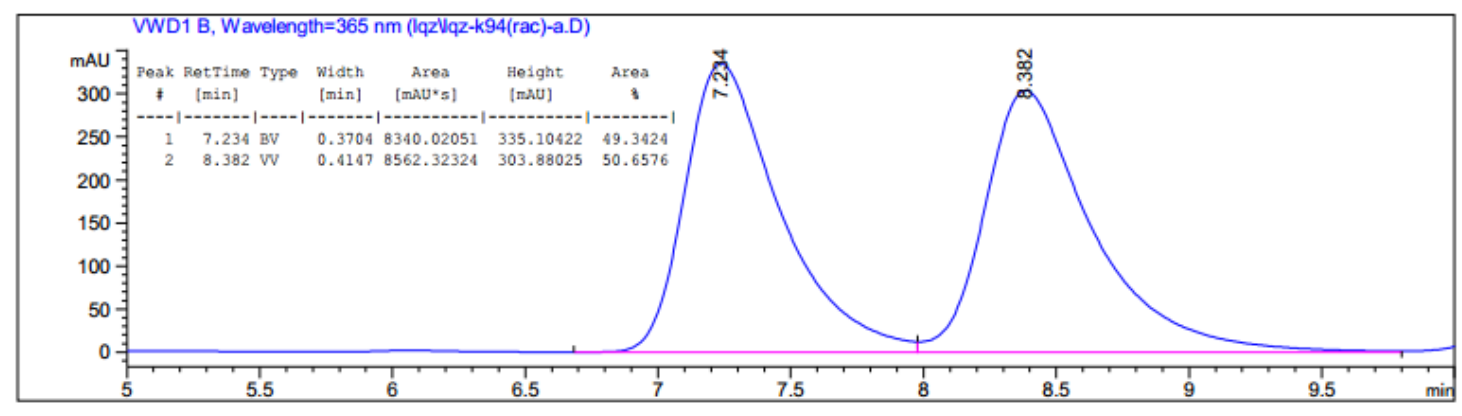


Chiral HPLC spectrum of 4 ha
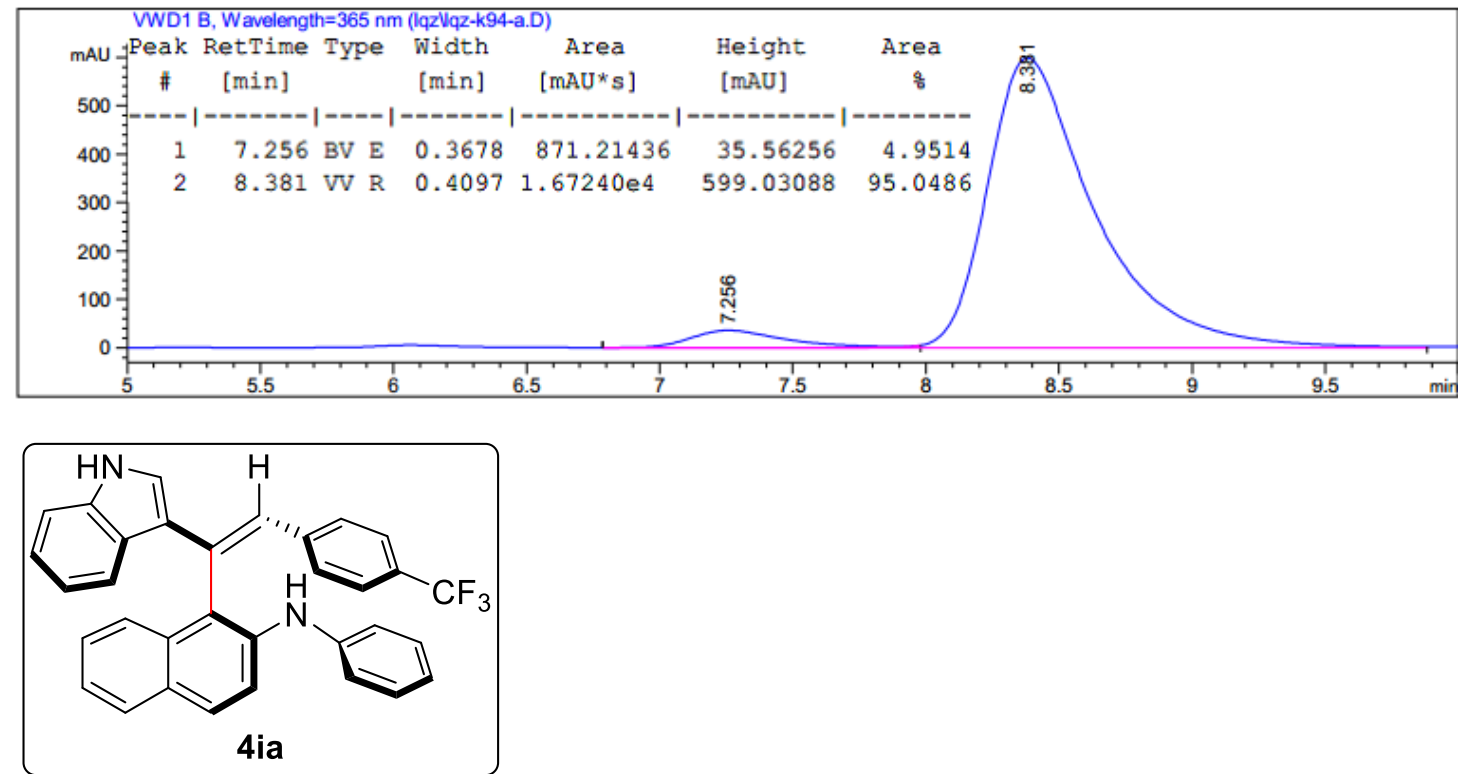

(aS)-(Z)-1-(1-(1H-indol-3-yl)-2-(4-(trifluoromethyl)phenyl)vinyl)-N-phenylnaphthalen-2-

\section{amine (4ia)}

According to procedure $\mathbf{B}$, 4ia was obtained as a yellow foam, $35.3 \mathrm{mg}$ (70\% yield, $91 \%$ ee, $\mathrm{Z}: \mathrm{E}=9: 1)$. ${ }^{1}$ H NMR (400 MHz, $\left.\mathbf{C D C l}_{3}\right) \delta 8.23-8.21(\mathrm{~m}, 1 \mathrm{H}), 8.09(\mathrm{~s}, 1 \mathrm{H}), 7.85-7.82(\mathrm{~m}, 2 \mathrm{H}), 7.77-7.75(\mathrm{~m}$, $1 \mathrm{H}), 7.73(\mathrm{~s}, 1 \mathrm{H}), 7.58(\mathrm{~d}, J=9.0 \mathrm{~Hz}, 1 \mathrm{H}), 7.44-7.41(\mathrm{~m}, 1 \mathrm{H}), 7.35-7.26(\mathrm{~m}, 6 \mathrm{H}), 7.19-7.15(\mathrm{~m}$, $2 \mathrm{H}), 7.08(\mathrm{~d}, J=8.2 \mathrm{~Hz}, 2 \mathrm{H}), 6.93-6.898(\mathrm{~m}, 1 \mathrm{H}), 6.85-6.83(\mathrm{~m}, 2 \mathrm{H}), 6.78(\mathrm{~d}, J=2.8 \mathrm{~Hz}, 1 \mathrm{H}), 6.07$ $(\mathrm{s}, 1 \mathrm{H})$.

${ }^{13} \mathbf{C}$ NMR (126 MHz, $\left.\mathbf{C D C l}_{3}\right) \delta 142.9,140.8,137.9,137.4,133.9,133.1,129.3,129.2,128.8,128.2$, $128.1,128.0,127.0,126.2,126.1,125.1,125.1,125.02,124.99,124.93,124.88,123.6,122.9,122.0$, $121.8,121.1,120.8,119.1,118.4,118.0,117.87,111.87$.

HRMS (ESI) calculated for $[\mathrm{M}+\mathrm{H}]^{+} \mathrm{C}_{33} \mathrm{H}_{24} \mathrm{~F}_{3} \mathrm{~N}_{2}{ }^{+}, \mathrm{m} / \mathrm{z}$ : 505.1892, found: 505.1890 .

M.P. $81-84{ }^{\circ} \mathrm{C}$.

$[\alpha]^{20}{ }_{\mathrm{D}}=-1.2^{\circ}\left(\mathrm{c}=0.5, \mathrm{CHCl}_{3}\right)$.

HPLC analysis: HPLC DAICEL CHIRALPAK AD-H, hexane/isopropyl alcohol $=70 / 30$, flow rate $=1$ $\mathrm{mL} / \mathrm{min}, \lambda=254 \mathrm{~nm}$ ), $\mathrm{tR}$ (major) $=6.2 \mathrm{~min}, \mathrm{tR}($ minor $)=4.4 \mathrm{~min}$, ee $=91 \%$.

Chiral HPLC spectrum of racemic 4ia

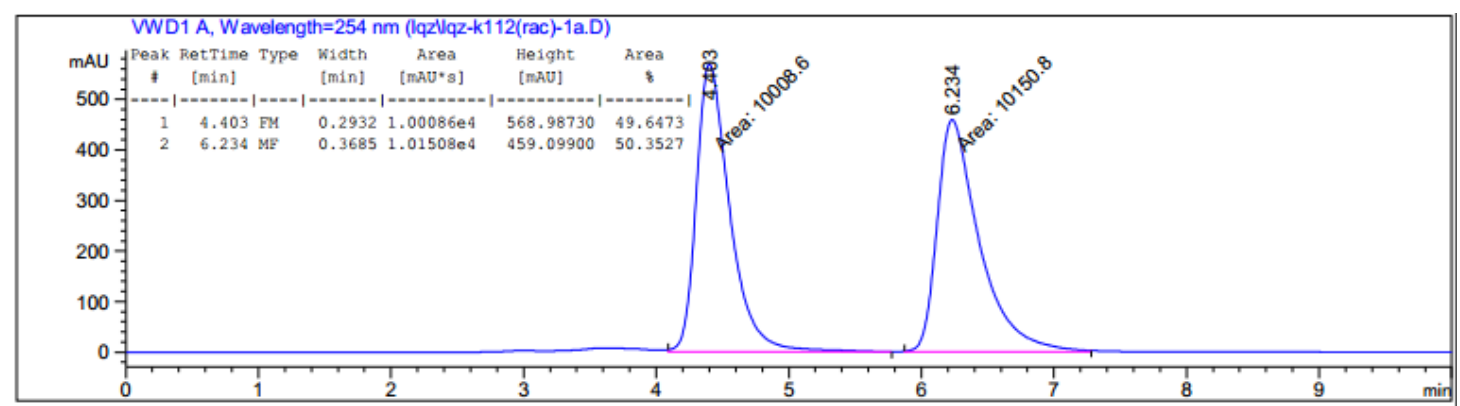

Chiral HPLC spectrum of 4ia 

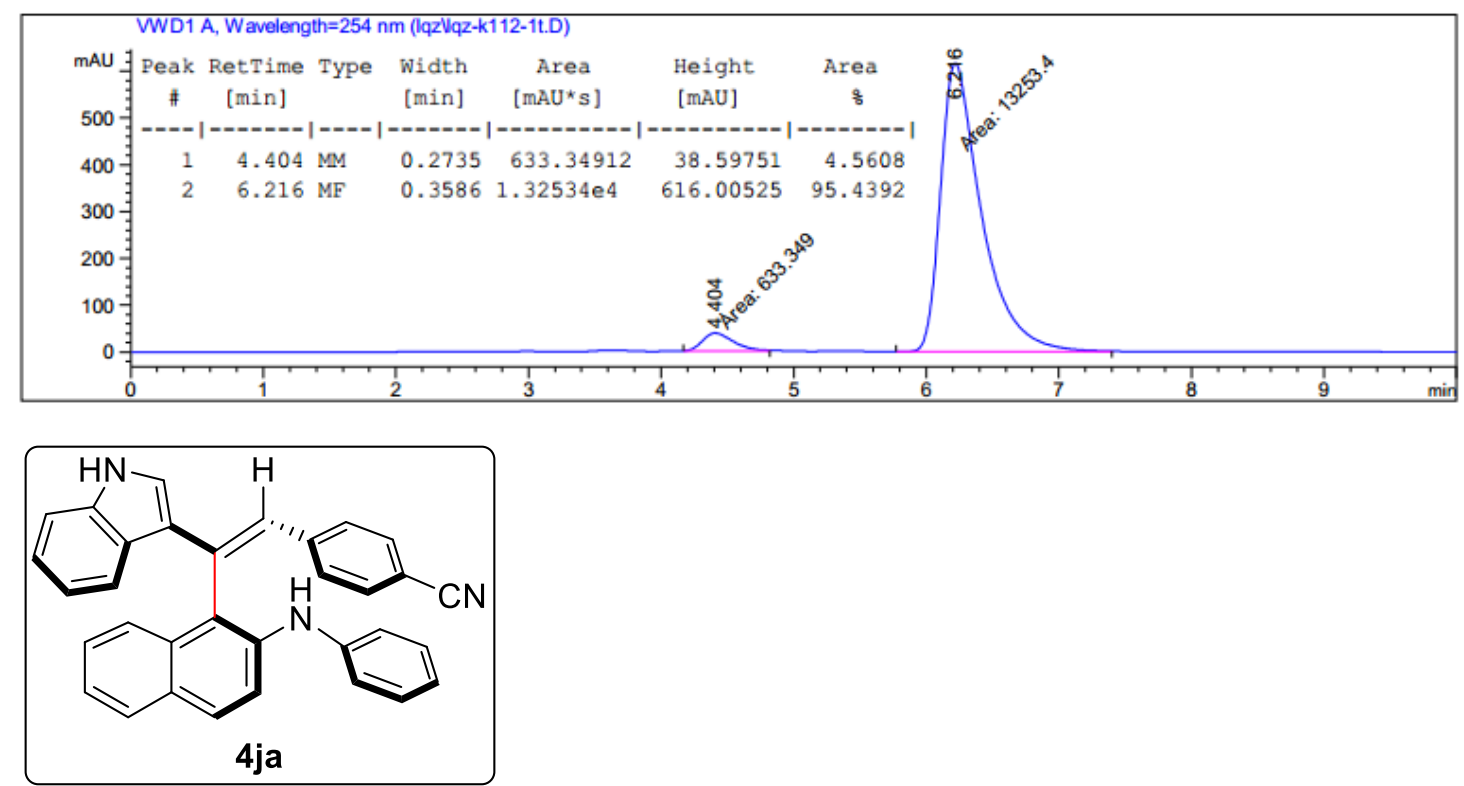

(aS)-(Z)-4-(2-(1H-indol-3-yl)-2-(2-(phenylamino)naphthalen-1-yl)vinyl)benzonitrile (4ja)

According to procedure $\mathbf{B}, \mathbf{4 j a}$ was obtained as a yellow oil, $40.1 \mathrm{mg}$ (87\% yield, $91 \%$ ee, $\mathrm{Z}: \mathrm{E}=11: 1)$.

${ }^{1}$ H NMR (400 MHz, $\left.\mathbf{C D C l}_{3}\right) \delta 8.22-8.18(\mathrm{~m}, 2 \mathrm{H}), 7.86-7.83(\mathrm{~m}, 2 \mathrm{H}), 7.74-7.70(\mathrm{~m}, 2 \mathrm{H}), 7.59$ (d, $J$ $=9.0 \mathrm{~Hz}, 1 \mathrm{H}), 7.45-7.40(\mathrm{~m}, 1 \mathrm{H}), 7.35-7.30(\mathrm{~m}, 4 \mathrm{H}), 7.28-7.26(\mathrm{~m}, 2 \mathrm{H}), 7.22-7.17(\mathrm{~m}, 2 \mathrm{H}), 7.08$ $-7.05(\mathrm{~m}, 2 \mathrm{H}), 6.96-6.91(\mathrm{~m}, 1 \mathrm{H}), 6.87-6.85(\mathrm{~m}, 2 \mathrm{H}), 6.78(\mathrm{~d}, J=2.7 \mathrm{~Hz}, 1 \mathrm{H}), 6.04(\mathrm{~s}, 1 \mathrm{H})$.

${ }^{13}$ C NMR (101 MHz, $\left.\mathbf{C D C l}_{3}\right) \delta 142.7,142.1,138.0,137.4,135.4,133.0,131.9,129.3,129.2,129.0$, $128.5,128.2,127.1,126.7,125.5,124.8,124.7,123.6,123.0,121.9,121.6,121.2,120.7,119.2,119.1$, 118.4, 117.8, 112.0, 109.3.

HRMS (ESI) calculated for $[\mathrm{M}+\mathrm{H}]^{+} \mathrm{C}_{33} \mathrm{H}_{24} \mathrm{~N}_{3}{ }^{+}, \mathrm{m} / \mathrm{z}: 462.1970$, found: 462.1968 .

M.P. $74-76^{\circ} \mathrm{C}$.

$[\alpha]^{20}{ }_{D}=-41^{\circ}\left(\mathrm{c}=0.2, \mathrm{CHCl}_{3}\right)$.

HPLC analysis: HPLC DAICEL CHIRALPAK AD-H, hexane/isopropyl alcohol $=70 / 30$, flow rate $=1$ $\mathrm{mL} / \mathrm{min}, \lambda=254 \mathrm{~nm}), \mathrm{tR}($ major $)=10.3 \mathrm{~min}, \mathrm{tR}(\operatorname{minor})=7.1 \mathrm{~min}, \mathrm{ee}=91 \%$.

Chiral HPLC spectrum of racemic 4ja

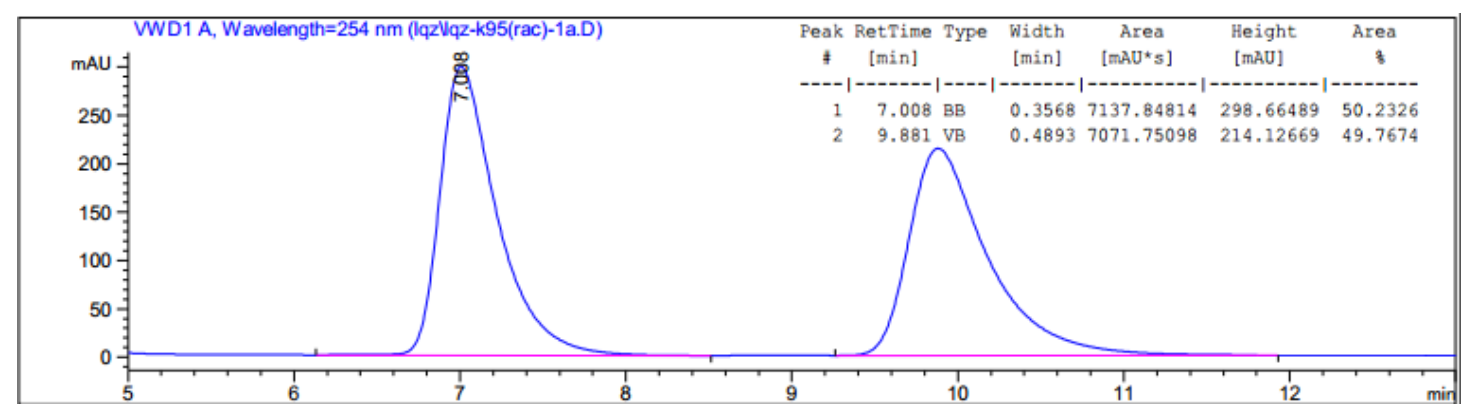

Chiral HPLC spectrum of $4 \mathbf{j a}$ 

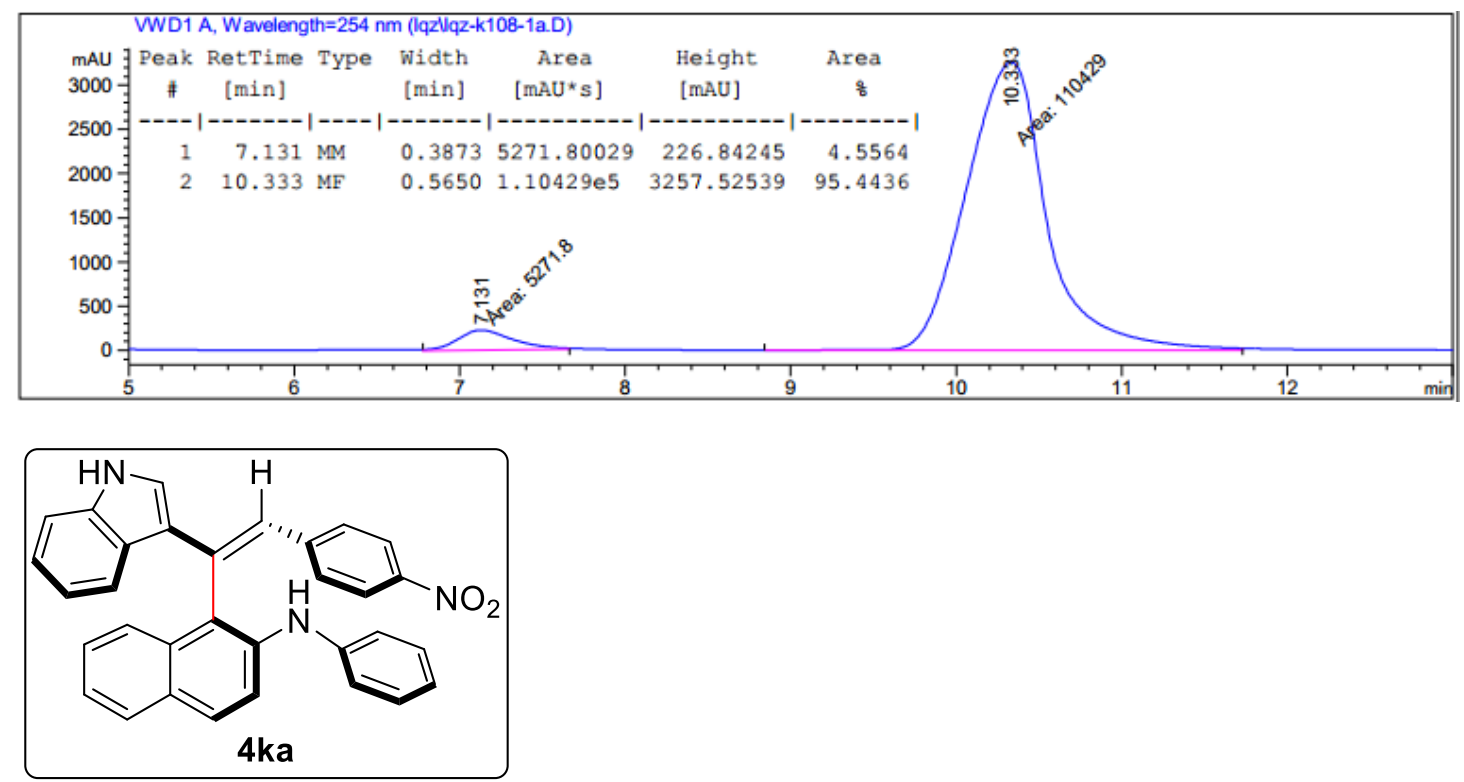

(aS)-(Z)-1-(1-(1H-indol-3-yl)-2-(4-nitrophenyl)vinyl)-N-phenylnaphthalen-2-amine (4ka) According to procedure $\mathbf{B}, \mathbf{4 k a}$ was obtained as a red solid, $35.1 \mathrm{mg}$ (73\% yield, $93 \%$ ee, $\mathrm{Z}: \mathrm{E}=9: 1)$. ${ }^{1}$ H NMR (500 MHz, CDCl $) \delta 8.23-8.20(\mathrm{~m}, 2 \mathrm{H}), 7.87-7.83(\mathrm{~m}, 4 \mathrm{H}), 7.75(\mathrm{~s}, 1 \mathrm{H}), 7.73-7.71(\mathrm{~m}$, $1 \mathrm{H}), 7.60(\mathrm{~d}, J=9.0 \mathrm{~Hz}, 1 \mathrm{H}), 7.44-7.42(\mathrm{~m}, 1 \mathrm{H}), 7.36-7.30(\mathrm{~m}, 4 \mathrm{H}), 7.17(\mathrm{dd}, J=8.5,7.3 \mathrm{~Hz}, 2 \mathrm{H})$, $7.10(\mathrm{~d}, J=8.9 \mathrm{~Hz}, 2 \mathrm{H}), 6.93-6.90(\mathrm{~m}, 1 \mathrm{H}), 6.89-6.85(\mathrm{~m}, 2 \mathrm{H}), 6.81$ (d, $J=2.7 \mathrm{~Hz}, 1 \mathrm{H}), 6.03(\mathrm{~s}, 1 \mathrm{H})$. ${ }^{13} \mathbf{C}$ NMR (126 MHz, CDCl $) \delta 145.6,144.2,142.6,138.0,137.5,136.2,133.0,129.3,129.2,129.1$, $128.5,128.2,127.1,127.0,125.0,124.8,124.6,123.7,123.5,123.1,121.9,121.5,121.3,120.8,119.1$, 118.4, 117.8, 112.0.

HRMS (ESI) calculated for $[\mathrm{M}+\mathrm{H}]^{+} \mathrm{C}_{32} \mathrm{H}_{24} \mathrm{~N}_{3} \mathrm{O}_{2}{ }^{+}, \mathrm{m} / \mathrm{z}$ : 482.1869 , found: 482.1867 .

M.P. $112-114^{\circ} \mathrm{C}$.

$[\alpha]^{20}{ }_{D}=-77^{\circ}\left(\mathrm{c}=0.2, \mathrm{CHCl}_{3}\right)$.

HPLC analysis: HPLC DAICEL CHIRALPAK AD-H, hexane/isopropyl alcohol $=70 / 30$, flow rate $=1$ $\mathrm{mL} / \mathrm{min}, \lambda=365 \mathrm{~nm}$ ), $\mathrm{tr}$ (major) $=9.9 \mathrm{~min}, \mathrm{tr}($ minor $)=8.1 \mathrm{~min}, \mathrm{ee}=93 \%$.

Chiral HPLC spectrum of racemic $\mathbf{4 k a}$

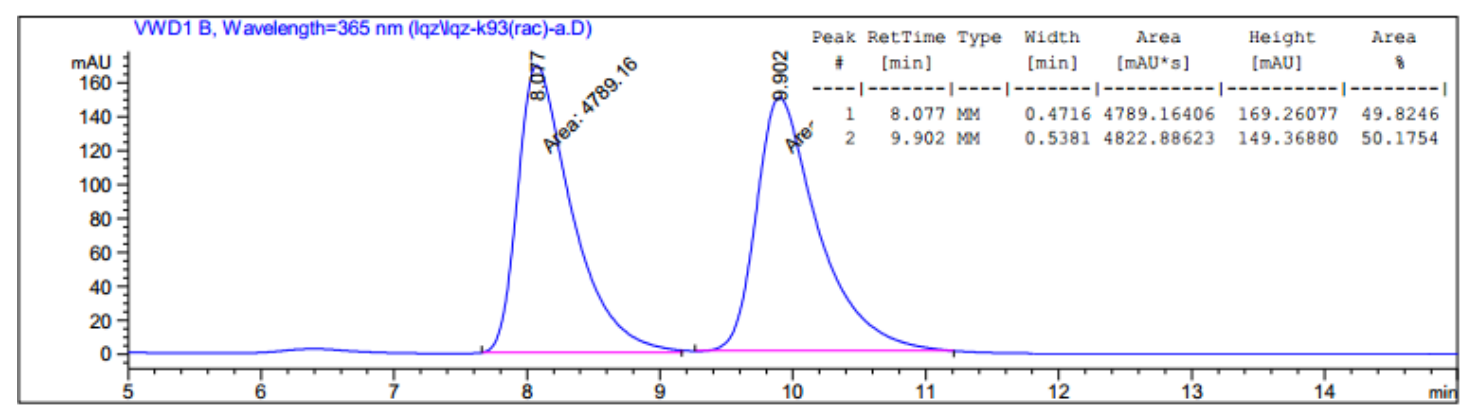

Chiral HPLC spectrum of $\mathbf{4 k a}$ 

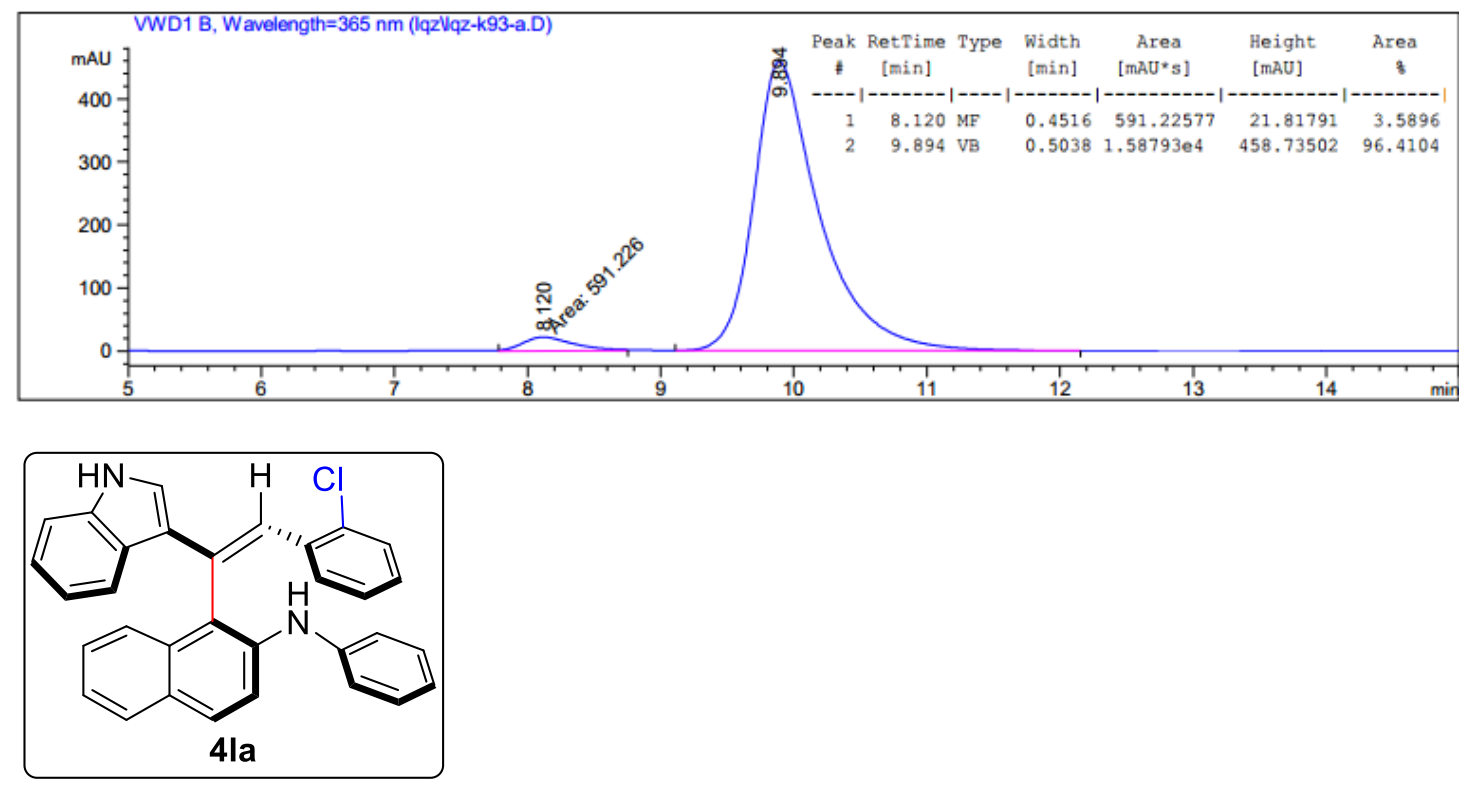

(aS)-(Z)-1-(2-(2-chlorophenyl)-1-(1H-indol-3-yl)vinyl)-N-phenylnaphthalen-2-amine (4la) According to procedure $\mathbf{B}, 4 \mathbf{l a}$ was obtained as a yellow oil, $20.2 \mathrm{mg}$ (43\% yield, 83\% ee, Z:E > 20:1). ${ }^{1}$ H NMR (500 MHz, CDCl $) \delta 8.24-8.22(\mathrm{~m}, 1 \mathrm{H}), 8.05(\mathrm{~s}, 1 \mathrm{H}), 7.89$ (s, 1H), $7.77(\mathrm{dd}, J=12.4,7.6$ $\mathrm{Hz}, 2 \mathrm{H}), 7.71(\mathrm{~d}, J=8.9 \mathrm{~Hz}, 1 \mathrm{H}), 7.47(\mathrm{~d}, J=8.9 \mathrm{~Hz}, 1 \mathrm{H}), 7.40-7.38(\mathrm{~m}, 1 \mathrm{H}), 7.30-7.27(\mathrm{~m}, 4 \mathrm{H})$, $7.20(\mathrm{~d}, J=8.0 \mathrm{~Hz}, 1 \mathrm{H}), 7.14(\mathrm{t}, J=7.5 \mathrm{~Hz}, 2 \mathrm{H}), 6.93(\mathrm{t}, J=7.7 \mathrm{~Hz}, 1 \mathrm{H}), 6.88(\mathrm{t}, J=7.4 \mathrm{~Hz}, 1 \mathrm{H}), 6.81$ (dd, $J=13.5,8.0 \mathrm{~Hz}, 3 \mathrm{H}), 6.74(\mathrm{~s}, 1 \mathrm{H}), 6.71(\mathrm{~s}, 1 \mathrm{H}), 6.18(\mathrm{~s}, 1 \mathrm{H})$.

${ }^{13}$ C NMR (126 MHz, CDCl $) \delta 143.0,138.0,137.2,135.5,133.8,133.4,133.2,129.2,129.00,128.98$, $128.95,128.3,128.0,127.8,126.7,126.1,125.9,125.3,125.2,124.8,123.1,122.7,121.8,121.4,121.0$, 120.9, 118.8, 118.2, 118.1, 111.6.

HRMS (ESI) calculated for $[\mathrm{M}+\mathrm{H}]^{+} \mathrm{C}_{32} \mathrm{H}_{24} \mathrm{ClN}_{2}{ }^{+}, \mathrm{m} / \mathrm{z}: 471.1628$, found: 471.1641 .

$[\alpha]^{20}{ }_{\mathrm{D}}=40^{\circ}\left(\mathrm{c}=0.2, \mathrm{CHCl}_{3}\right)$.

HPLC analysis: HPLC DAICEL CHIRALPAK AD-H, hexane/isopropyl alcohol $=70 / 30$, flow rate $=1$ $\mathrm{mL} / \mathrm{min}, \lambda=365 \mathrm{~nm}), \mathrm{tR}($ major $)=15.8 \mathrm{~min}, \mathrm{tR}(\operatorname{minor})=4.4 \mathrm{~min}, \mathrm{ee}=83 \%$.

Chiral HPLC spectrum of racemic 4la

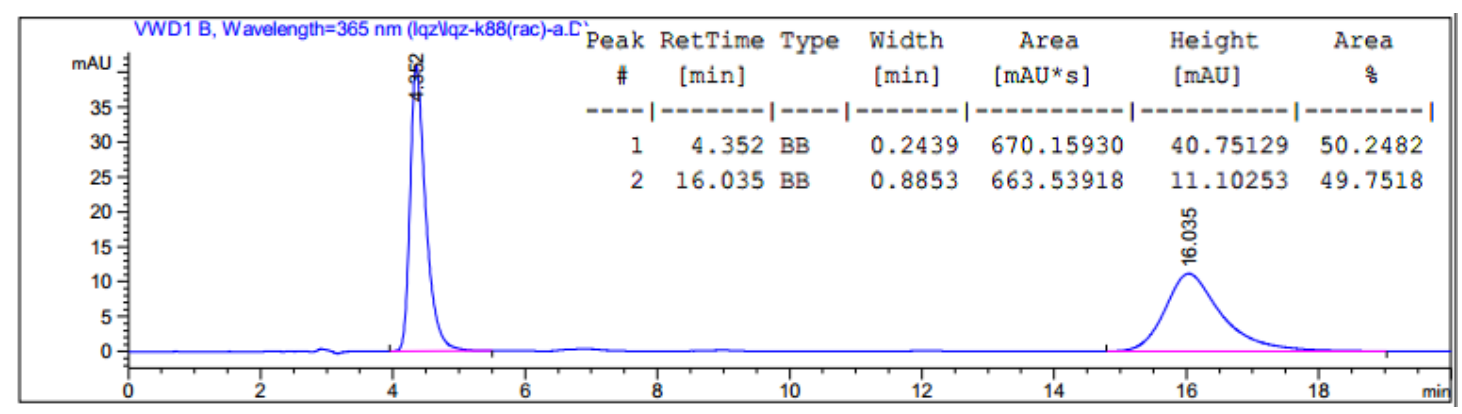

Chiral HPLC spectrum of 4 la 

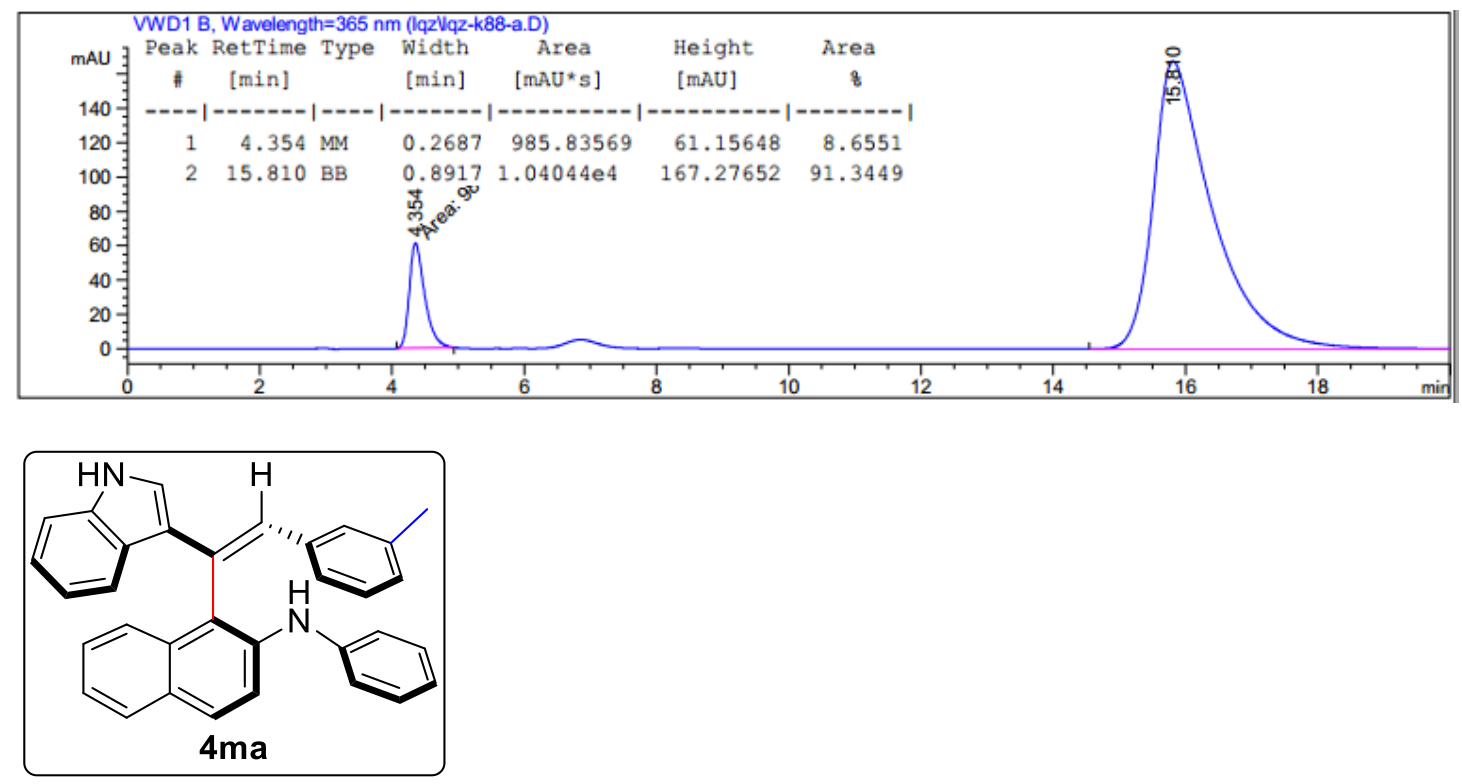

(aS)-(Z)-1-(1-(1H-indol-3-yl)-2-(m-tolyl)vinyl)-N-phenylnaphthalen-2-amine (4ma)

According to procedure B, 4ma was obtained as a light brown foam, $28.8 \mathrm{mg}$ (64\% yield, 93\% ee, Z:E =11:1).

${ }^{1}$ H NMR (600 MHz, CDCl $) \delta 8.17(\mathrm{~s}, 1 \mathrm{H}), 7.86(\mathrm{~s}, 1 \mathrm{H}), 7.77-7.74(\mathrm{~m}, 3 \mathrm{H}), 7.64(\mathrm{~s}, 1 \mathrm{H}), 7.55(\mathrm{~d}, J=$ $8.9 \mathrm{~Hz}, 1 \mathrm{H}), 7.29-7.21(\mathrm{~m}, 5 \mathrm{H}), 7.13-7.10(\mathrm{~m}, 2 \mathrm{H}), 6.87-6.83(\mathrm{~m}, 5 \mathrm{H}), 6.81(\mathrm{~d}, J=7.4 \mathrm{~Hz}, 1 \mathrm{H})$, $6.73(\mathrm{~d}, J=7.6 \mathrm{~Hz}, 1 \mathrm{H}), 6.61(\mathrm{~d}, J=2.7 \mathrm{~Hz}, 1 \mathrm{H}), 6.14(\mathrm{~s}, 1 \mathrm{H}), 2.07$ (s, 3H).

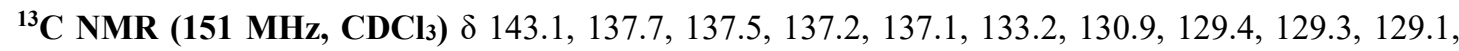
$128.3,128.1,128.0,127.9,127.5,126.6,125.3,125.2,125.0,124.9,123.3,123.0,122.5,121.4,120.8$, 120.6, 119.0, 118.4, 118.2, 111.7, 21.3.

HRMS (ESI) calculated for $[\mathrm{M}+\mathrm{H}]^{+} \mathrm{C}_{33} \mathrm{H}_{27} \mathrm{~N}_{2}{ }^{+}, \mathrm{m} / \mathrm{z}: 451.2174$, found: 451.2171 .

M.P. $68-70^{\circ} \mathrm{C}$.

$[\alpha]^{20}{ }_{\mathrm{D}}=-80^{\circ}\left(\mathrm{c}=0.2, \mathrm{CHCl}_{3}\right)$.

HPLC analysis: HPLC DAICEL CHIRALPAK AD-H, hexane/isopropyl alcohol = 70/30, flow rate = 1 $\mathrm{mL} / \mathrm{min}, \lambda=365 \mathrm{~nm})$, tR (major) $=16.4 \mathrm{~min}, \mathrm{tR}($ minor $)=4.4 \mathrm{~min}$, ee $=93 \%$.

Chiral HPLC spectrum of racemic 4 ma

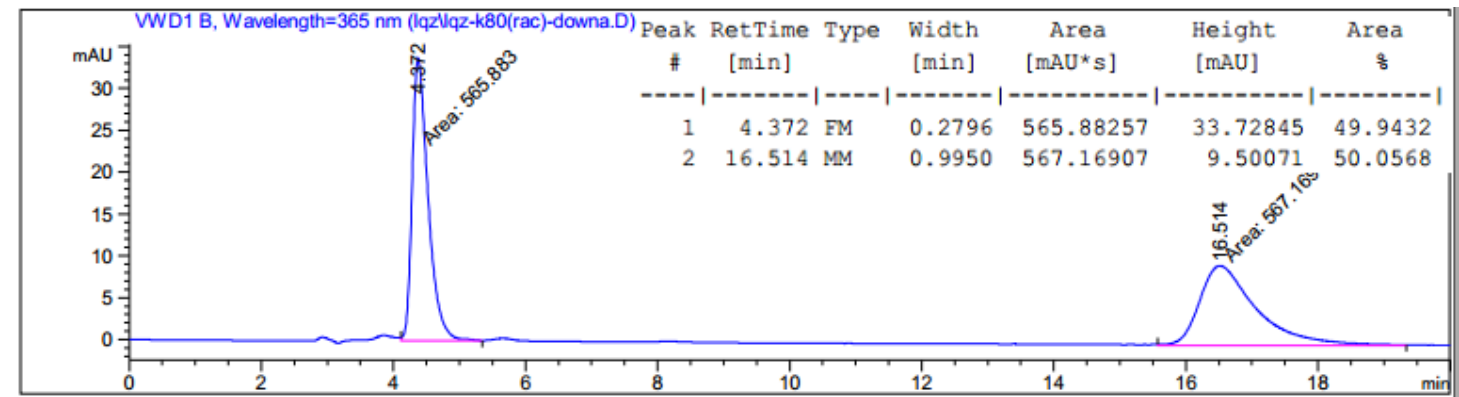

Chiral HPLC spectrum of $\mathbf{4 m a}$ 

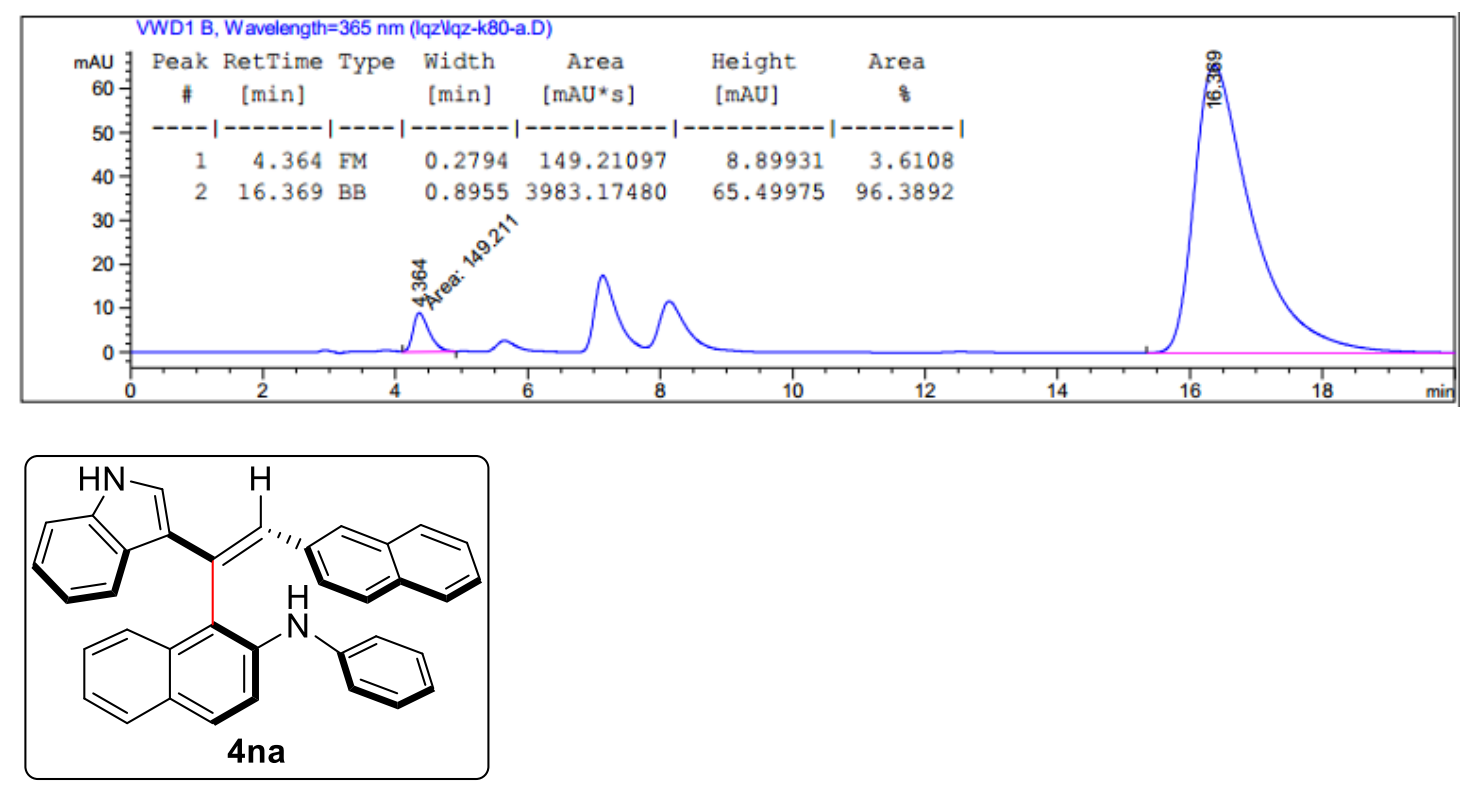

(aS)-(Z)-1-(1-(1H-indol-3-yl)-2-(naphthalen-2-yl)vinyl)-N-phenylnaphthalen-2-amine (4na) According to procedure B, 4na was obtained as a yellowish solid, $23.3 \mathrm{mg}$ (48\% yield, 93\% ee, Z:E = 10:1).

${ }^{1}$ H NMR (400 MHz, CDCl $) \delta 8.24(\mathrm{~d}, J=7.4 \mathrm{~Hz}, 1 \mathrm{H}), 7.91(\mathrm{~s}, 1 \mathrm{H}), 7.84(\mathrm{~s}, 1 \mathrm{H}), 7.81-7.77(\mathrm{dm}, 3 \mathrm{H})$, $7.60-7.56(\mathrm{~m}, 2 \mathrm{H}), 7.51(\mathrm{~s}, 1 \mathrm{H}), 7.49-7.46(\mathrm{~m}, 1 \mathrm{H}), 7.40(\mathrm{~d}, J=8.7 \mathrm{~Hz}, 1 \mathrm{H}), 7.33-7.27(\mathrm{~m}, 5 \mathrm{H})$, $7.25-7.20(\mathrm{~m}, 2 \mathrm{H}), 7.05(\mathrm{t}, J=7.7 \mathrm{~Hz}, 3 \mathrm{H}), 6.83-6.78(\mathrm{~m}, 3 \mathrm{H}), 6.69(\mathrm{~d}, J=2.7 \mathrm{~Hz}, 1 \mathrm{H}), 6.18(\mathrm{~s}, 1 \mathrm{H})$. ${ }^{13}$ C NMR (101 MHz, CDCl $) \delta 143.0,138.0,137.3,134.9,133.4,133.3,132.3,131.7,129.3,129.1$, $128.5,128.00,127.98,127.9,127.8,127.6,127.4,126.8,126.0,125.8,125.6,125.5,125.2,125.1,123.4$, $122.9,122.7,121.5,120.9,120.8,119.0,118.5,118.3,111.8$.

HRMS (ESI) calculated for $[\mathrm{M}+\mathrm{H}]^{+} \mathrm{C}_{36} \mathrm{H}_{27} \mathrm{~N}_{2}{ }^{+}, \mathrm{m} / \mathrm{z}: 487.2174$, found: 487.2178 .

M.P. $90-93^{\circ} \mathrm{C}$.

$[\alpha]^{20}{ }_{\mathrm{D}}=-149^{\circ}\left(\mathrm{c}=0.2, \mathrm{CHCl}_{3}\right)$.

HPLC analysis: HPLC DAICEL CHIRALPAK AD-H, hexane/isopropyl alcohol = 70/30, flow rate = 1 $\mathrm{mL} / \mathrm{min}, \lambda=365 \mathrm{~nm}), \mathrm{tR}($ major $)=11.3 \mathrm{~min}, \mathrm{tR}($ minor $)=8.6 \mathrm{~min}$, ee $=93 \%$.

Chiral HPLC spectrum of racemic 4na

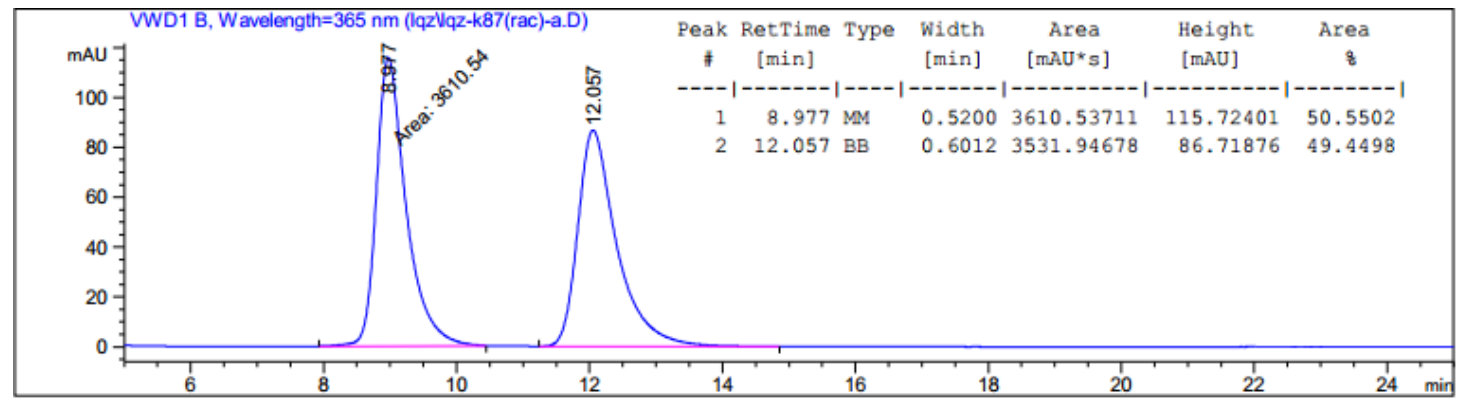

Chiral HPLC spectrum of 4na 

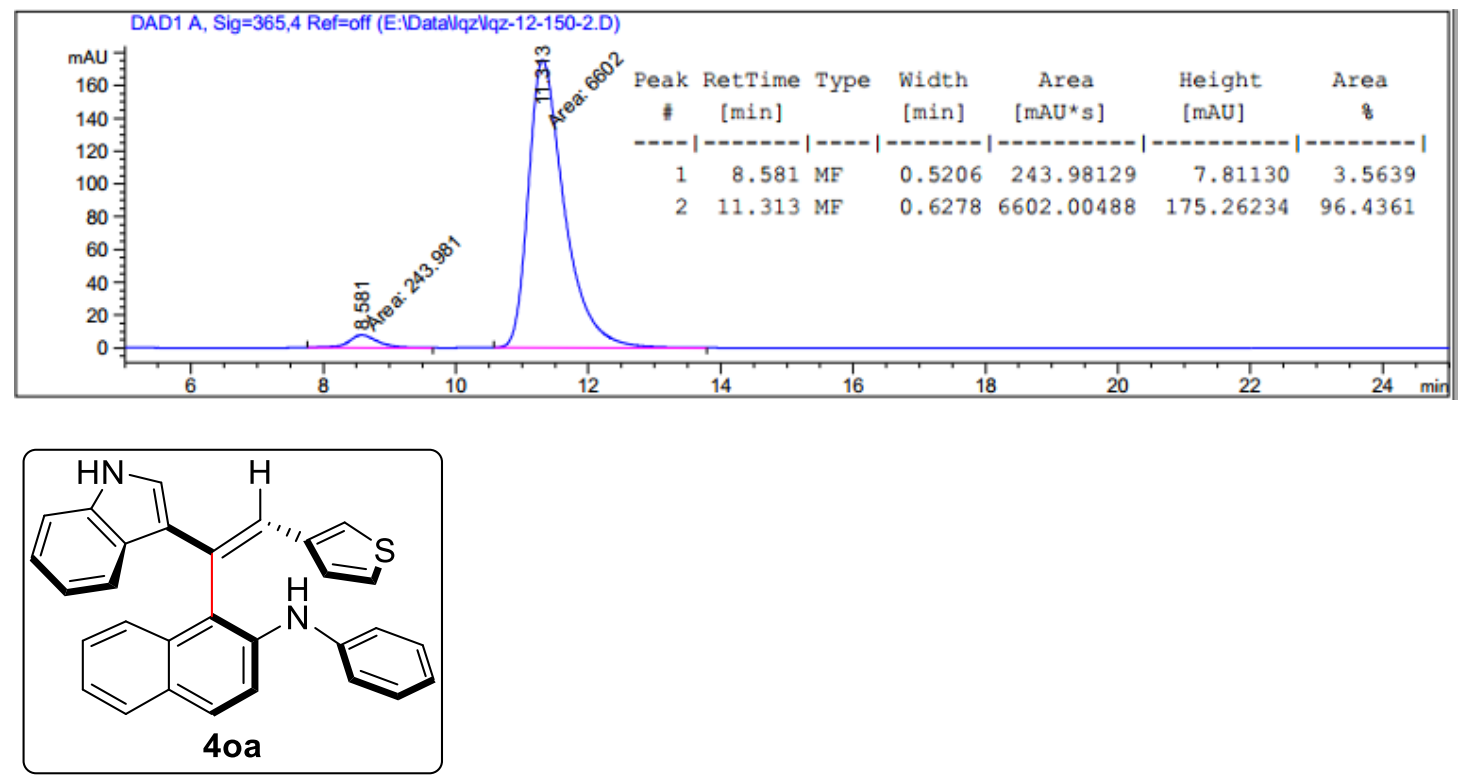

(aS)-(Z)-1-(1-(1H-indol-3-yl)-2-(thiophen-3-yl)vinyl)-N-phenylnaphthalen-2-amine (40a)

According to procedure $\mathbf{B}, \mathbf{4 0 a}$ was obtained as a yellow solid, $32.8 \mathrm{mg}$ (74\% yield, $92 \%$ ee, $\mathrm{Z}: \mathrm{E}=7: 1)$. ${ }^{1}$ H NMR (400 MHz, CDCl $) \delta 8.24-8.22(\mathrm{~m}, 1 \mathrm{H}), 7.99(\mathrm{~s}, 1 \mathrm{H}), 7.87-7.82(\mathrm{~m}, 2 \mathrm{H}), 7.79-7.77(\mathrm{~m}$, 2H), $7.66(\mathrm{~d}, J=9.0 \mathrm{~Hz}, 1 \mathrm{H}), 7.41-7.37(\mathrm{~m}, 1 \mathrm{H}), 7.34-7.29(\mathrm{~m}, 4 \mathrm{H}), 7.22-7.18(\mathrm{~m}, 2 \mathrm{H}), 7.00$ (dd, $J$ $=5.1,3.0 \mathrm{~Hz}, 1 \mathrm{H}), 6.97-6.90(\mathrm{~m}, 3 \mathrm{H}), 6.76-6.74(\mathrm{~m}, 2 \mathrm{H}), 6.57(\mathrm{dd}, J=5.0,1.3 \mathrm{~Hz}, 1 \mathrm{H}), 6.20(\mathrm{~s}, 1 \mathrm{H})$. ${ }^{13} \mathbf{C}$ NMR (126 MHz, $\left.\mathbf{C D C l}_{3}\right) \delta 143.0,138.7,138.0,137.3,133.0,129.9,129.3,129.2,128.5,128.2$, $127.9,127.8,126.8,125.4,125.0,124.8,123.4,123.0,122.8,122.6,121.9,121.6,120.8,120.7,119.3$, 118.2, 117.9, 111.7 .

HRMS (ESI) calculated for $[\mathrm{M}+\mathrm{H}]^{+} \mathrm{C}_{30} \mathrm{H}_{23} \mathrm{~N}_{2} \mathrm{~S}^{+}, \mathrm{m} / \mathrm{z}: 443.1582$, found: 443.1579 .

M.P. $77-79^{\circ} \mathrm{C}$.

$[\alpha]^{20}{ }_{\mathrm{D}}=-53^{\circ}\left(\mathrm{c}=0.2, \mathrm{CHCl}_{3}\right)$.

HPLC analysis: HPLC DAICEL CHIRALPAK AD-H, hexane/isopropyl alcohol $=70 / 30$, flow rate $=1$ $\mathrm{mL} / \mathrm{min}, \lambda=365 \mathrm{~nm}$ ), $\operatorname{tr}($ major $)=15.6 \mathrm{~min}, \mathrm{tR}(\operatorname{minor})=6.0 \mathrm{~min}, \mathrm{ee}=92 \%$.

Chiral HPLC spectrum of racemic 4 oa

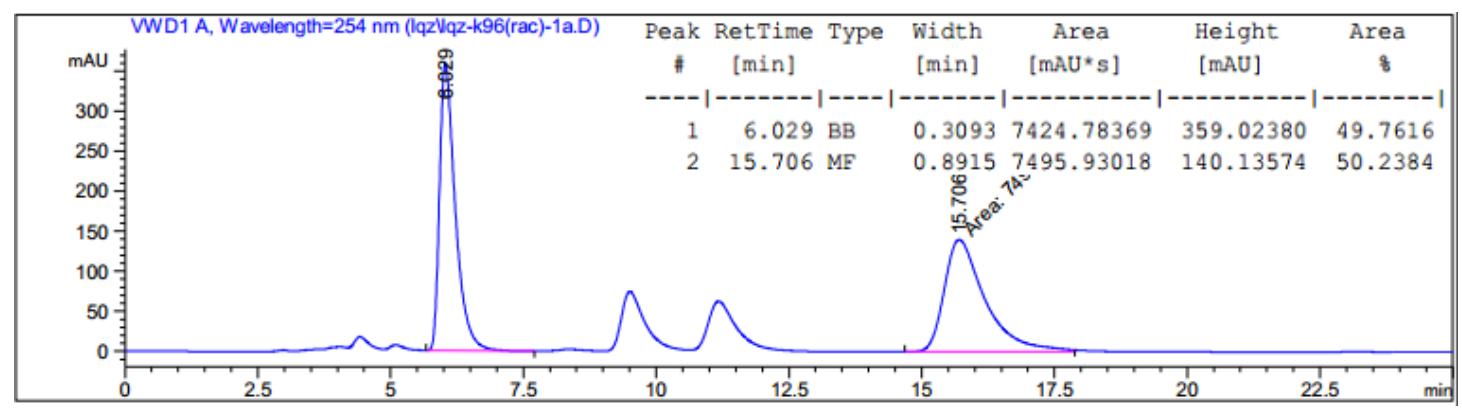

Chiral HPLC spectrum of $\mathbf{4 o a}$ 

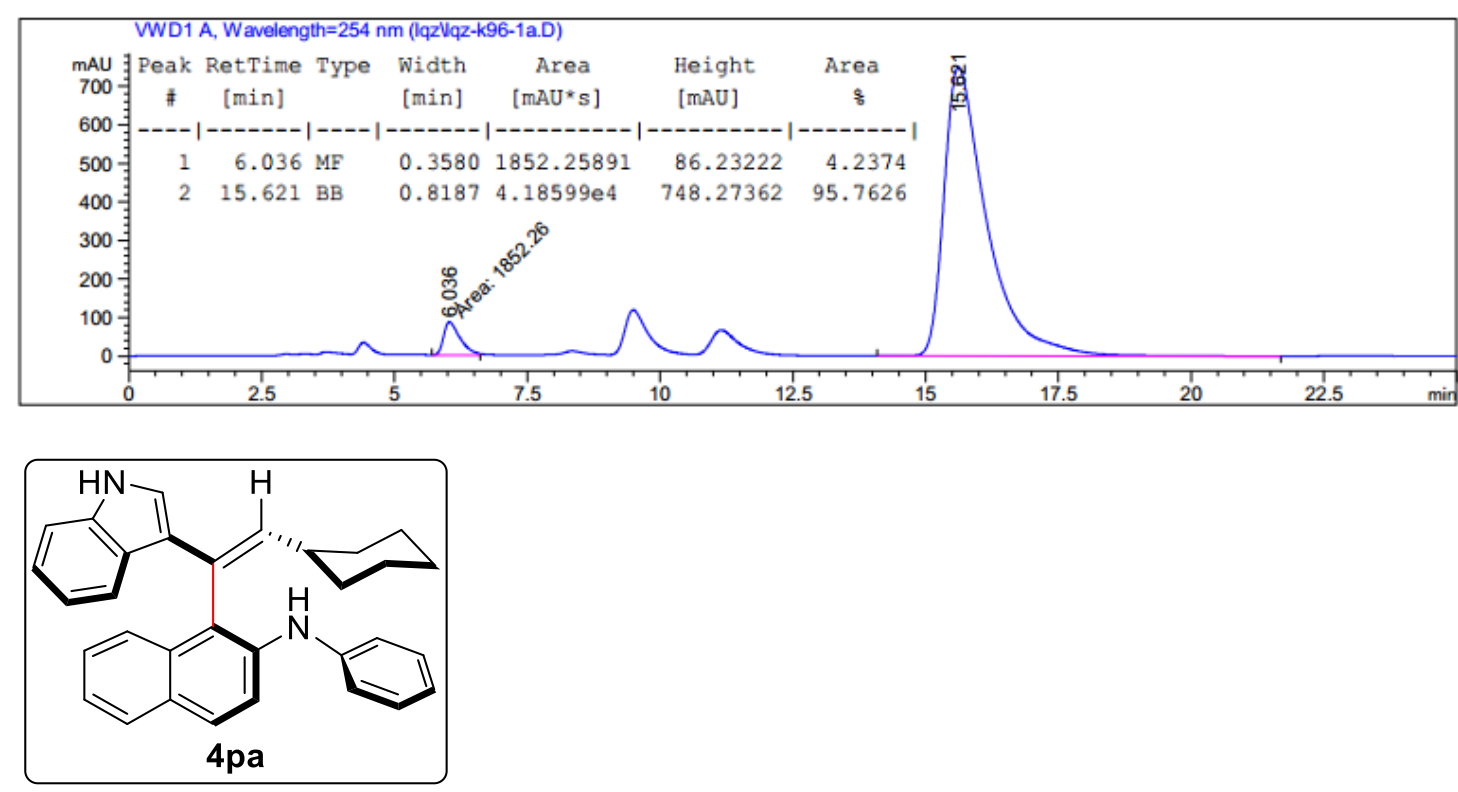

(aS)-(Z)-1-(2-cyclohexyl-1-(1H-indol-3-yl)vinyl)-N-phenylnaphthalen-2-amine (4pa)

According to procedure $\mathbf{B}, \mathbf{4}$ pa was obtained as a white solid, $37.1 \mathrm{mg}$ (84\% yield, 70\% ee, Z:E > 20:1). ${ }^{1}$ H NMR (400 MHz, CDCl $) \delta 8.15-8.13(\mathrm{~m}, 1 \mathrm{H}), 7.87(\mathrm{~s}, 1 \mathrm{H}), 7.82-7.79(\mathrm{~m}, 3 \mathrm{H}), 7.66(\mathrm{~d}, J=8.9$ $\mathrm{Hz}, 1 \mathrm{H}), 7.36-7.29(\mathrm{~m}, 3 \mathrm{H}), 7.28-7.24(\mathrm{~m}, 4 \mathrm{H}), 7.08-7.06(\mathrm{~m}, 2 \mathrm{H}), 6.97-6.93(\mathrm{~m}, 1 \mathrm{H}), 6.63(\mathrm{~d}, J$ $=9.9 \mathrm{~Hz}, 1 \mathrm{H}), 6.55(\mathrm{~d}, J=2.6 \mathrm{~Hz}, 1 \mathrm{H}), 6.20(\mathrm{~s}, 1 \mathrm{H}), 1.96-1.87(\mathrm{~m}, 1 \mathrm{H}), 1.59-1.51(\mathrm{~m}, 4 \mathrm{H}), 1.30-$ $1.10(\mathrm{~m}, 4 \mathrm{H}), 1.07-0.93(\mathrm{~m}, 2 \mathrm{H})$.

${ }^{13} \mathbf{C}$ NMR (101 MHz, $\left.\mathbf{C D C l}_{3}\right) \delta 143.6,137.5,137.2,136.3,133.4,129.3,129.2,127.8,127.7,127.4$, 126.2, 125.4, 125.2, 124.4, 123.9, 123.2, 122.4, 121.3, 120.7, 120.4, 118.50, 118.45, 117.2, 111.5, 38.7, $33.4,33.1,26.0,25.5,25.5$.

HRMS (ESI) calculated for $[\mathrm{M}+\mathrm{H}]^{+} \mathrm{C}_{32} \mathrm{H}_{31} \mathrm{~N}_{2}{ }^{+}, \mathrm{m} / \mathrm{z}: 443.2487$, found: 443.2489 .

M.P. $72-74{ }^{\circ} \mathrm{C}$.

$[\alpha]^{20}{ }_{\mathrm{D}}=99^{\circ}\left(\mathrm{c}=0.2, \mathrm{CHCl}_{3}\right)$.

HPLC analysis: HPLC DAICEL CHIRALPAK AD-H, hexane/isopropyl alcohol $=70 / 30$, flow rate $=1$ $\mathrm{mL} / \mathrm{min}, \lambda=365 \mathrm{~nm}$ ), $\mathrm{tR}$ (major) $=5.9 \mathrm{~min}, \mathrm{tR}($ minor $)=3.6 \mathrm{~min}$, ee $=70 \%$.

Chiral HPLC spectrum of racemic 4 pa

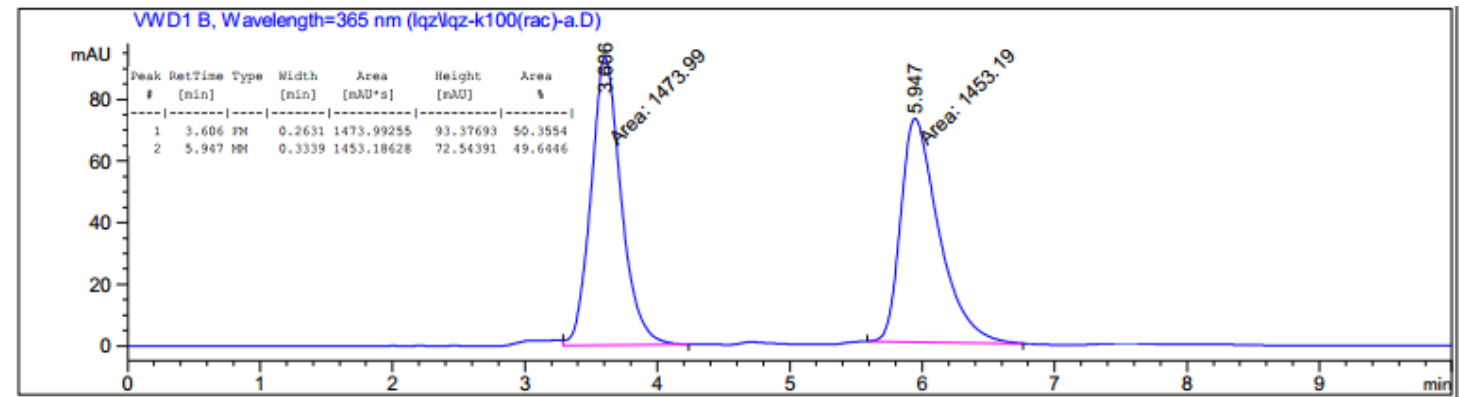

Chiral HPLC spectrum of $4 p a$ 

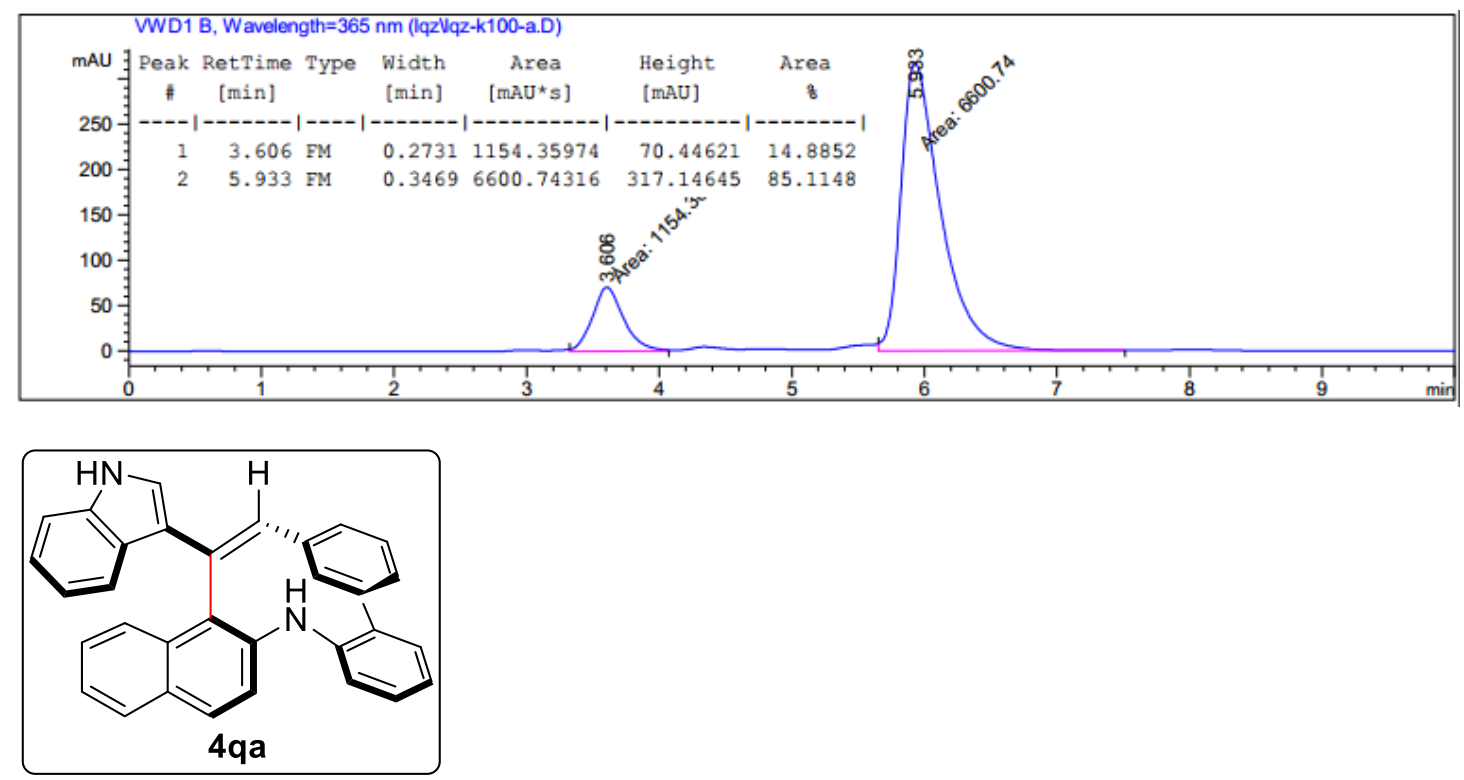

(aS)-(Z)-1-(1-(1H-indol-3-yl)-2-phenylvinyl)-N-(o-tolyl)naphthalen-2-amine (4qa)

According to procedure $\mathbf{B}, \mathbf{4 q a}$ was obtained as a white solid, $36.9 \mathrm{mg}$ ( $82 \%$ yield, $91 \%$ ee, $\mathrm{Z}: \mathrm{E}>20: 1)$. ${ }^{1}$ H NMR (400 MHz, $\left.\mathbf{C D C l}_{3}\right) \delta 8.22-8.20(\mathrm{~m}, 1 \mathrm{H}), 8.01(\mathrm{~s}, 1 \mathrm{H}), 7.82-7.78(\mathrm{~m}, 3 \mathrm{H}), 7.73(\mathrm{~s}, 1 \mathrm{H}), 7.41$ $-7.38(\mathrm{~m}, 2 \mathrm{H}), 7.31-7.29$ (m, 2H), 7.27 (d, $J=3.3 \mathrm{~Hz}, 1 \mathrm{H}), 7.11-7.06$ (m, 8H), 6.90 (td, $J=7.2,1.6$ $\mathrm{Hz}, 1 \mathrm{H}), 6.77(\mathrm{~d}, J=2.7 \mathrm{~Hz}, 1 \mathrm{H}), 6.03(\mathrm{~s}, 1 \mathrm{H}), 1.85(\mathrm{~s}, 3 \mathrm{H})$.

${ }^{13} \mathbf{C}$ NMR (101 MHz, $\left.\mathbf{C D C l}_{3}\right) \delta 140.8,138.2,137.3,137.2,133.3,131.4,130.8,129.6,128.9,128.4$, $128.3,128.1,127.9,126.8,126.7,126.5,125.3,125.2,125.1,122.9,122.6,122.3,121.4,120.73,120.70$, 119.7, 118.7, 117.3, 111.7, 17.4.

HRMS (ESI) calculated for $[\mathrm{M}+\mathrm{H}]^{+} \mathrm{C}_{33} \mathrm{H}_{27} \mathrm{~N}_{2}{ }^{+}, \mathrm{m} / \mathrm{z}: 451.2174$, found: 451.2173 .

M.P. $76-78^{\circ} \mathrm{C}$.

$[\alpha]^{20}{ }_{\mathrm{D}}=-84^{\circ}\left(\mathrm{c}=0.2, \mathrm{CHCl}_{3}\right)$.

HPLC analysis: HPLC DAICEL CHIRALPAK AD-H, hexane/isopropyl alcohol $=70 / 30$, flow rate $=1$ $\mathrm{mL} / \mathrm{min}, \lambda=365 \mathrm{~nm}$ ), tR (major) $=7.0 \mathrm{~min}, \mathrm{tr}($ minor $)=4.9 \mathrm{~min}$, ee $=91 \%$.

Chiral HPLC spectrum of racemic $4 q a$

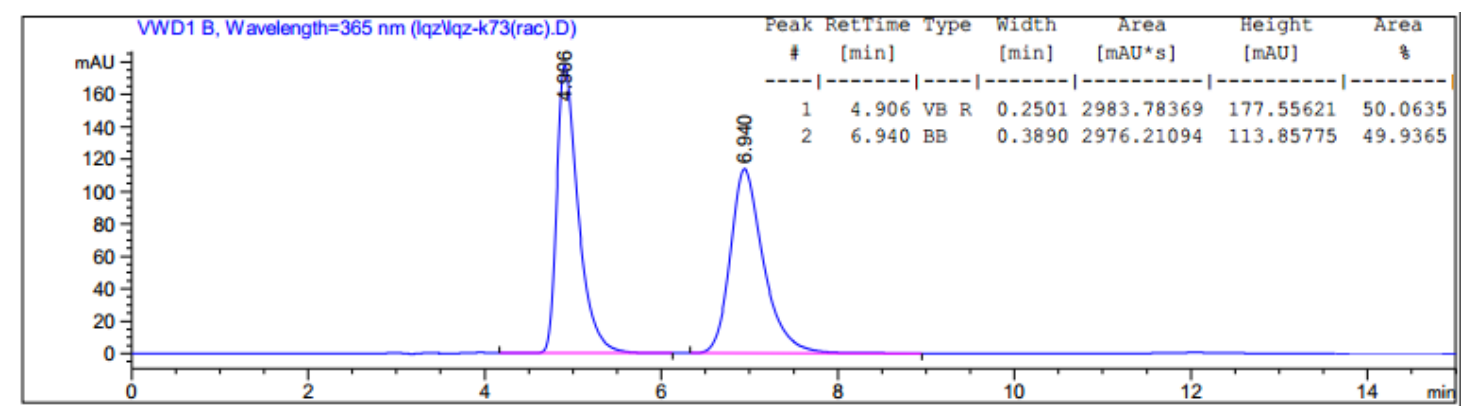

Chiral HPLC spectrum of $\mathbf{4 q a}$ 

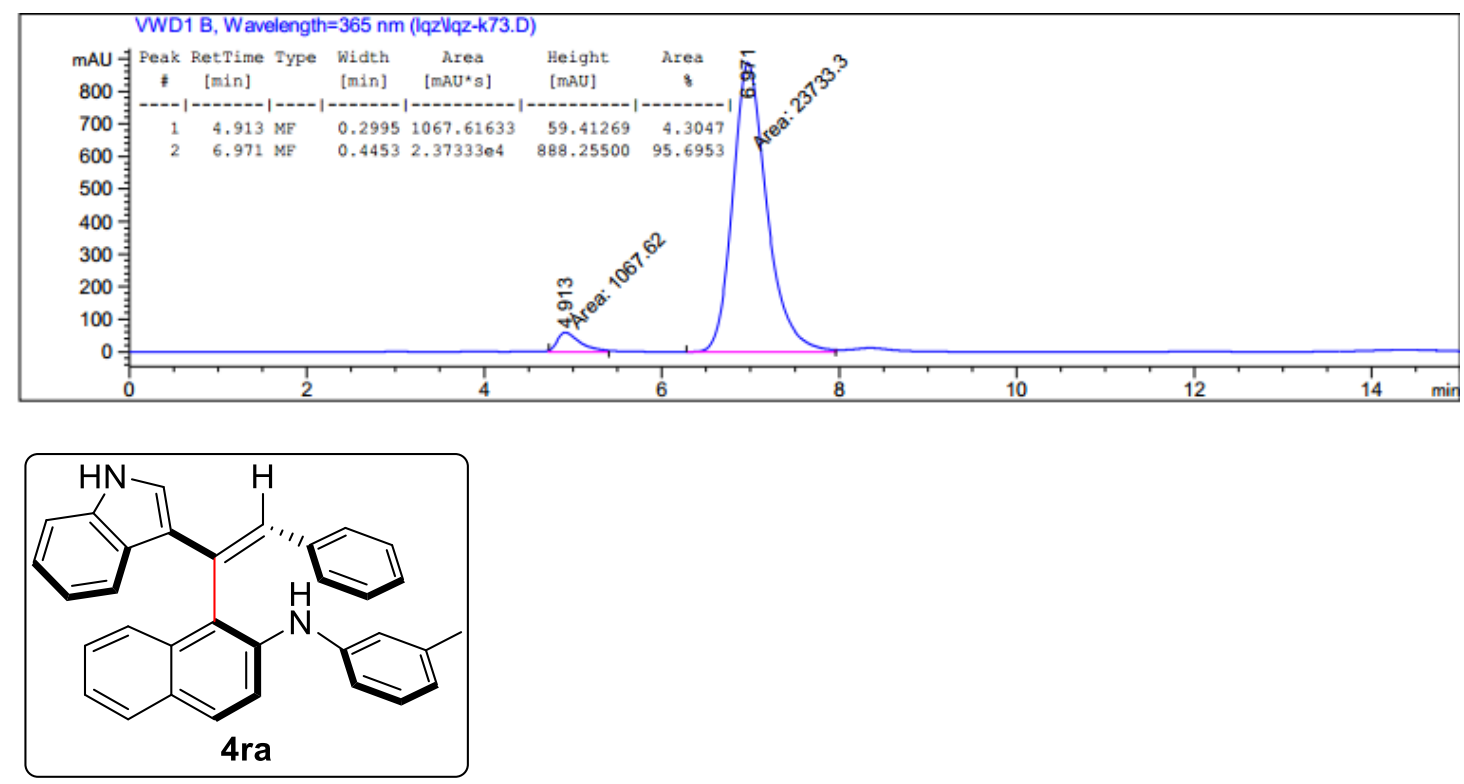

(aS)-(Z)-1-(1-(1H-indol-3-yl)-2-phenylvinyl)-N-(m-tolyl)naphthalen-2-amine (4ra)

According to procedure $\mathbf{B}$, 4ra was obtained as a yellow solid, $42.8 \mathrm{mg}$ (95\% yield, $90 \%$ ee, $\mathrm{Z}: \mathrm{E}=9: 1)$. ${ }^{1}$ H NMR (400 MHz, $\left.\mathbf{C D C l}_{3}\right) \delta 8.19-8.17(\mathrm{~m}, 1 \mathrm{H}), 7.95(\mathrm{~s}, 1 \mathrm{H}), 7.78-7.74(\mathrm{~m}, 3 \mathrm{H}), 7.68(\mathrm{~s}, 1 \mathrm{H}), 7.55$ (d, $J=9.0 \mathrm{~Hz}, 1 \mathrm{H}), 7.34-7.32(\mathrm{~m}, 1 \mathrm{H}), 7.28-7.22(\mathrm{~m}, 5 \mathrm{H}), 7.04-6.99(\mathrm{~m}, 6 \mathrm{H}), 6.70-6.67(\mathrm{~m}, 3 \mathrm{H})$, $6.62-6.61(\mathrm{~m}, 1 \mathrm{H}), 6.11(\mathrm{~s}, 1 \mathrm{H}), 2.19(\mathrm{~s}, 3 \mathrm{H})$.

${ }^{13}$ C NMR (101 MHz, $\left.\mathbf{C D C l}_{3}\right) \delta 143.0,139.0,138.0,137.29,137.25,133.2,131.3,129.2,128.9,128.4$, $128.3,128.2,128.0,127.9,126.70,126.68,125.4,125.2,125.1,123.3,122.62,122.55,122.5,120.8$, 120.7, 120.1, 118.5, 118.3, 116.3, 111.7, 21.4.

HRMS (ESI) calculated for $[\mathrm{M}+\mathrm{H}]^{+} \mathrm{C}_{33} \mathrm{H}_{27} \mathrm{~N}_{2}{ }^{+}, \mathrm{m} / \mathrm{z}: 451.2174$, found: 451.2173 .

M.P. $74-76^{\circ} \mathrm{C}$.

$[\alpha]^{20}{ }_{D}=-81.6^{\circ}\left(\mathrm{c}=1, \mathrm{CHCl}_{3}\right)$.

HPLC analysis: HPLC DAICEL CHIRALPAK AD-H, hexane/isopropyl alcohol $=70 / 30$, flow rate $=1$ $\mathrm{mL} / \mathrm{min}, \lambda=254 \mathrm{~nm}), \mathrm{tR}($ major $)=8.1 \mathrm{~min}, \mathrm{tR}($ minor $)=4.5 \mathrm{~min}$, ee $=90 \%$.

Chiral HPLC spectrum of racemic 4ra

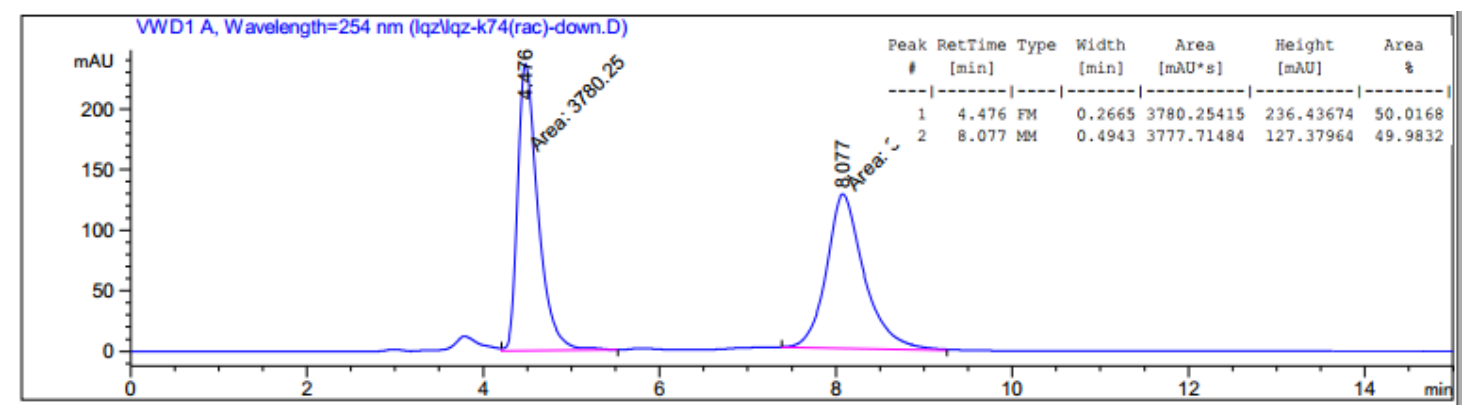

Chiral HPLC spectrum of $4 r a$ 

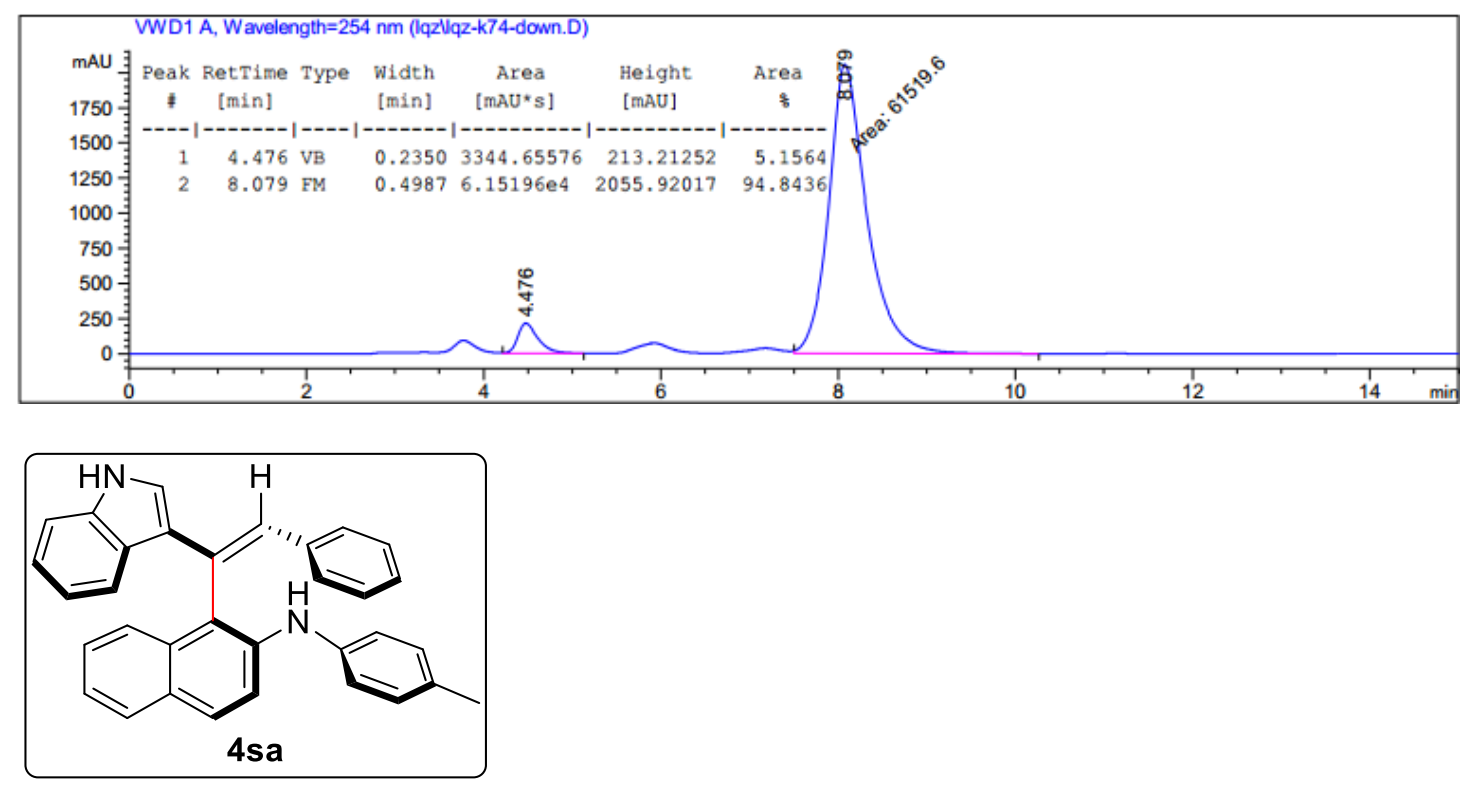

(aS)-(Z)-1-(1-(1H-indol-3-yl)-2-phenylvinyl)-N-(p-tolyl)naphthalen-2-amine (4sa)

According to procedure $\mathbf{B}, \mathbf{4} \mathbf{s a}$ was obtained as a yellow solid, $41.4 \mathrm{mg}$ (92\% yield, $90 \%$ ee, $\mathrm{Z}: \mathrm{E}=17: 1)$. ${ }^{1}$ H NMR (400 MHz, $\left.\mathbf{C D C l}_{3}\right) \delta 8.25-8.22(\mathrm{~m}, 1 \mathrm{H}), 7.98(\mathrm{~s}, 1 \mathrm{H}), 7.81-7.78(\mathrm{~m}, 3 \mathrm{H}), 7.73(\mathrm{~s}, 1 \mathrm{H}), 7.52$ (d, $J=9.0 \mathrm{~Hz}, 1 \mathrm{H}), 7.39-7.37$ (m, 1H), $7.32-7.29$ (m, 2H), $7.28-7.26(\mathrm{~m}, 2 \mathrm{H}), 7.07$ (s, 5H), $7.01-$ $6.99(\mathrm{~m}, 2 \mathrm{H}), 6.82-6.79(\mathrm{~m}, 2 \mathrm{H}), 6.73(\mathrm{~d}, J=2.7 \mathrm{~Hz}, 1 \mathrm{H}), 6.13(\mathrm{~s}, 1 \mathrm{H}), 2.28(\mathrm{~s}, 3 \mathrm{H})$.

${ }^{13} \mathbf{C}$ NMR (101 MHz, $\left.\mathbf{C D C l}_{3}\right) \delta 140.3,138.7,137.3,133.3,131.5,131.3,129.7,129.0,128.4,128.3$, 128.2, 128.0, 127.9, 126.7, 125.4, 125.2, 125.0, 123.0, 122.6, 121.7, 120.8, 120.7, 120.5, 118.5, 117.6, 111.7, 20.7.

HRMS (ESI) calculated for $[\mathrm{M}+\mathrm{H}]^{+} \mathrm{C}_{33} \mathrm{H}_{27} \mathrm{~N}_{2}{ }^{+}, \mathrm{m} / \mathrm{z}: 451.2174$, found: 451.2171 .

M.P. $83-86^{\circ} \mathrm{C}$.

$[\alpha]^{20}{ }_{\mathrm{D}}=-52^{\circ}\left(\mathrm{c}=0.2, \mathrm{CHCl}_{3}\right)$.

HPLC analysis: HPLC DAICEL CHIRALPAK AD-H, hexane/isopropyl alcohol $=60 / 40$, flow rate $=1$ $\mathrm{mL} / \mathrm{min}, \lambda=254 \mathrm{~nm}), \mathrm{tR}($ major $)=44.8 \mathrm{~min}, \mathrm{tR}(\operatorname{minor})=7.8 \mathrm{~min}, \mathrm{ee}=90 \%$.

Chiral HPLC spectrum of racemic $4 \mathbf{s a}$

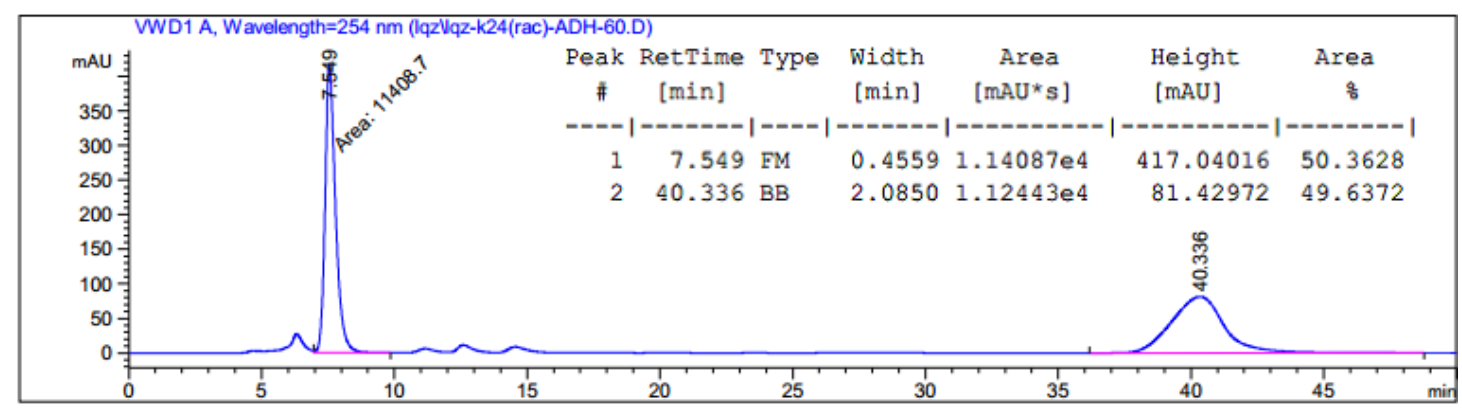

Chiral HPLC spectrum of $4 \mathrm{sa}$ 

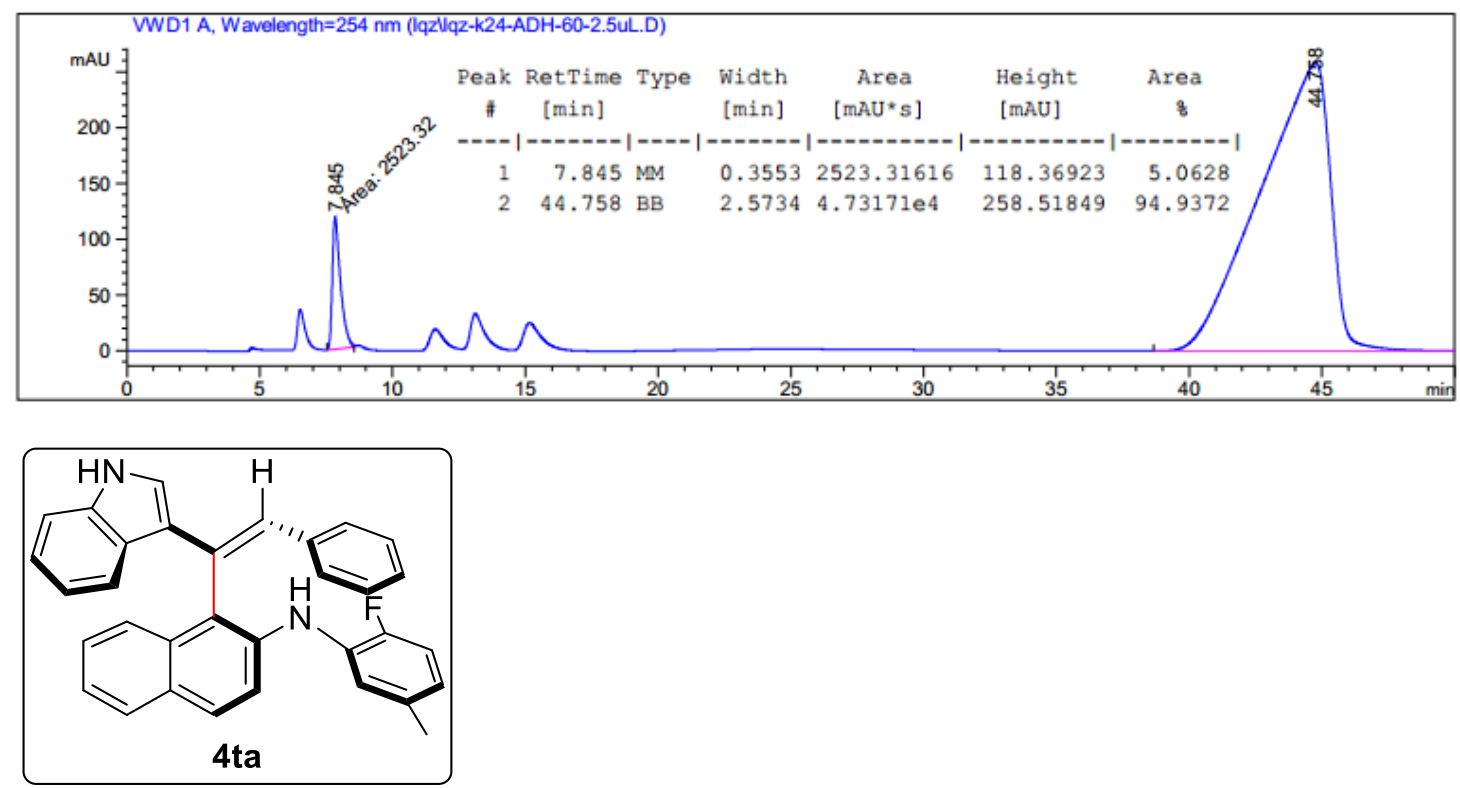

(aS)-(Z)-1-(1-(1H-indol-3-yl)-2-phenylvinyl)-N-(2-fluoro-5-methylphenyl)naphthalen-2amine (4ta)

According to procedure B, 4ta was obtained as a yellow oil, $22.0 \mathrm{mg}$ (47\% yield, 93\% ee, $\mathrm{Z}: \mathrm{E}=13: 1)$. ${ }^{1}$ H NMR (400 MHz, CDCl $) \delta 8.21-8.17(\mathrm{~m}, 1 \mathrm{H}), 8.04(\mathrm{~s}, 1 \mathrm{H}), 7.87-7.81(\mathrm{~m}, 4 \mathrm{H}), 7.71(\mathrm{~s}, 1 \mathrm{H}), 7.51$ $(\mathrm{d}, J=8.9 \mathrm{~Hz}, 1 \mathrm{H}), 7.41-7.38(\mathrm{~m}, 1 \mathrm{H}), 7.34-7.27(\mathrm{~m}, 4 \mathrm{H}), 7.05-6.99(\mathrm{~m}, 5 \mathrm{H}), 6.87-6.80(\mathrm{~m}, 2 \mathrm{H})$, $6.75(\mathrm{~d}, J=2.7 \mathrm{~Hz}, 1 \mathrm{H}), 6.62-6.58(\mathrm{~m}, 1 \mathrm{H}), 6.10(\mathrm{~s}, 1 \mathrm{H}), 2.19(\mathrm{~s}, 3 \mathrm{H})$.

${ }^{13}$ C NMR (101 MHz, CDCl $) \delta 153.5,151.1,137.24,137.17,136.9,133.6,133.3,131.1,130.9,130.8$, $129.5,128.4,128.23,128.20,128.1,127.9,126.7,126.6,125.4,125.3,125.2,123.8,123.6,122.6,121.8$, $121.8,120.8,120.7,119.3,119.3,118.6,118.5,115.1,114.9,111.6,20.9$.

HRMS (ESI) calculated for $[\mathrm{M}+\mathrm{H}]^{+} \mathrm{C}_{33} \mathrm{H}_{26} \mathrm{FN}_{2}{ }^{+}, \mathrm{m} / \mathrm{z}: 469.2080$, found: 469.2076.

$[\alpha]^{20}{ }_{\mathrm{D}}=3^{\circ}\left(\mathrm{c}=0.2, \mathrm{CHCl}_{3}\right)$.

HPLC analysis: HPLC DAICEL CHIRALPAK AD-H, hexane/isopropyl alcohol = 70/30, flow rate $=1$ $\mathrm{mL} / \mathrm{min}, \lambda=254 \mathrm{~nm}), \mathrm{tR}($ major $)=10.8 \mathrm{~min}, \mathrm{tR}($ minor $)=4.8 \mathrm{~min}$, ee $=93 \%$.

Chiral HPLC spectrum of racemic 4ta

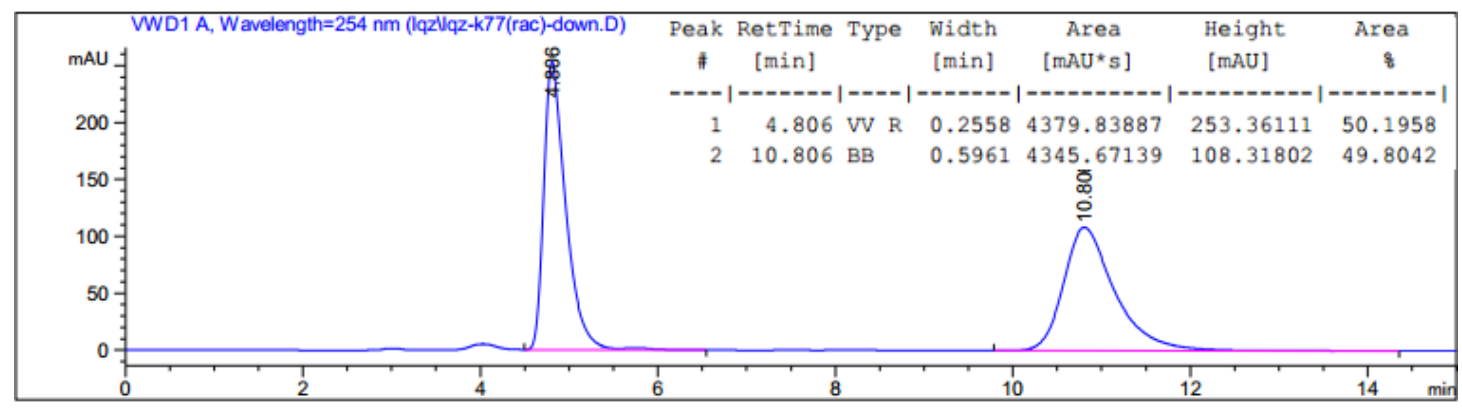

Chiral HPLC spectrum of $\mathbf{4 t a}$ 

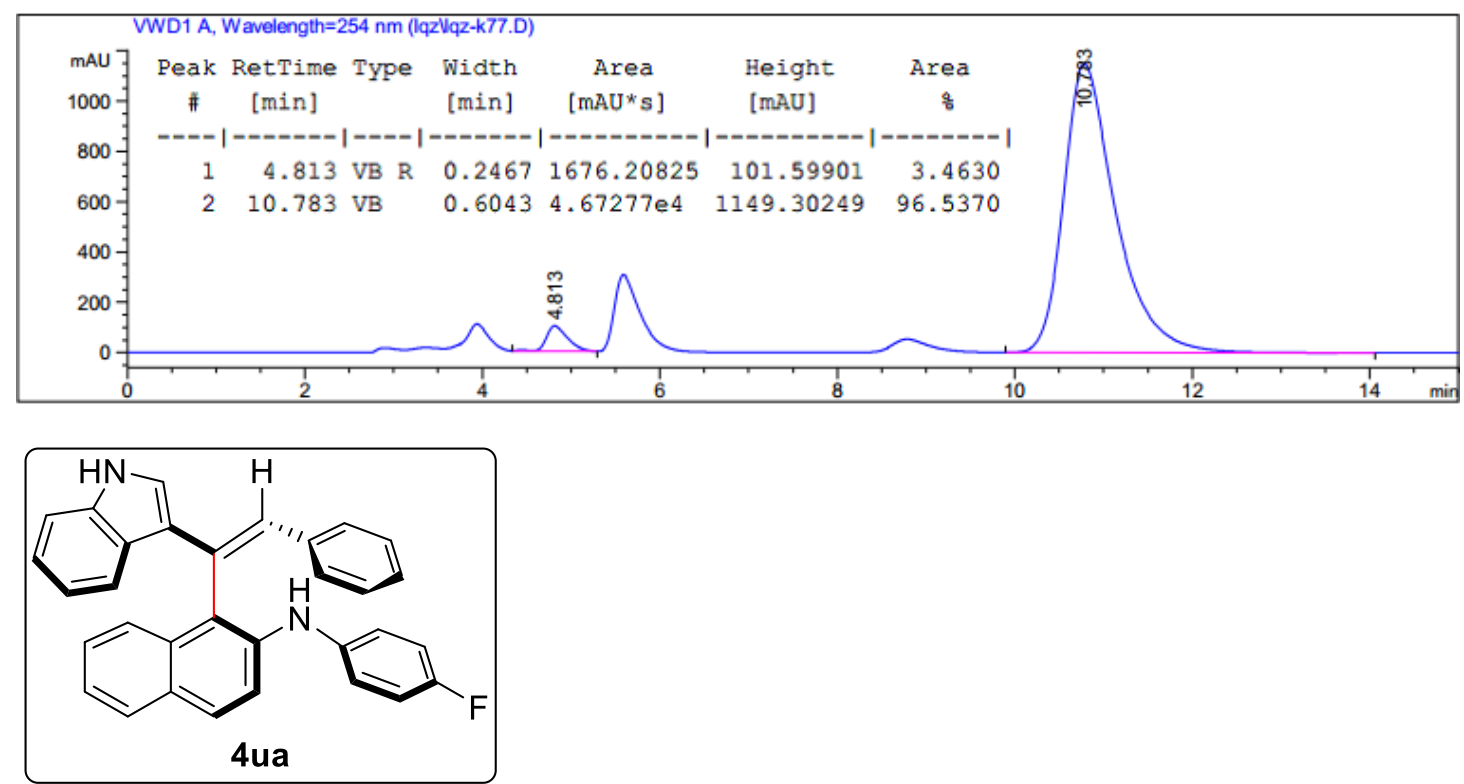

(aS)-(Z)-1-(1-(1H-indol-3-yl)-2-phenylvinyl)-N-(4-fluorophenyl)naphthalen-2-amine (4ua)

According to procedure $\mathbf{B}, 4$ ua was obtained as a yellow solid, $39.0 \mathrm{mg}$ ( $86 \%$ yield, $92 \%$ ee, $\mathrm{Z}: \mathrm{E}=12: 1)$. ${ }^{1}$ H NMR (400 MHz, $\left.\mathbf{C D C l}_{3}\right) \delta 8.23-8.21(\mathrm{~m}, 1 \mathrm{H}), 8.02(\mathrm{~s}, 1 \mathrm{H}), 7.82-7.79(\mathrm{~m}, 3 \mathrm{H}), 7.73(\mathrm{~s}, 1 \mathrm{H}), 7.42$ $-7.38(\mathrm{~m}, 2 \mathrm{H}), 7.32-7.28(\mathrm{~m}, 4 \mathrm{H}), 7.08-7.02(\mathrm{~m}, 5 \mathrm{H}), 6.89-6.84(\mathrm{~m}, 2 \mathrm{H}), 6.80-6.77(\mathrm{~m}, 2 \mathrm{H}), 6.73$ (d, $J=2.7 \mathrm{~Hz}, 1 \mathrm{H}), 6.07$ (s, 1H).

${ }^{13}$ C NMR (101 MHz, $\left.\mathbf{C D C l}_{3}\right) \delta$ 159.7, 157.3, 139.08, 139.05, 138.6, 137.3, 137.2, 133.3, 131.2, 129.1, 128.6, 128.24, 128.21, 127.99, 127.95, 126.8, 126.7, 125.4, 125.14, 125.11, 123.2, 122.8, 122.2, 122.1, $121.9,120.82,120.78,118.5,117.5,115.8,115.6,111.7$.

HRMS (ESI) calculated for $[\mathrm{M}+\mathrm{H}]^{+} \mathrm{C}_{32} \mathrm{H}_{24} \mathrm{FN}_{2}{ }^{+}$, m/z: $455.1924 \mathrm{C}_{32} \mathrm{H}_{24} \mathrm{FN}_{2}$, found: 455.1920 .

M.P. $90-92^{\circ} \mathrm{C}$.

$[\alpha]^{20}{ }_{D}=-11^{\circ}\left(\mathrm{c}=0.2, \mathrm{CHCl}_{3}\right)$.

HPLC analysis: HPLC DAICEL CHIRALPAK AD-H, hexane/isopropyl alcohol $=60 / 40$, flow rate $=1$ $\mathrm{mL} / \mathrm{min}, \lambda=365 \mathrm{~nm}$ ), $\operatorname{tr}($ major $)=25.7 \mathrm{~min}, \mathrm{tR}(\operatorname{minor})=7.2 \mathrm{~min}, \mathrm{ee}=92 \%$.

Chiral HPLC spectrum of racemic 4 ua

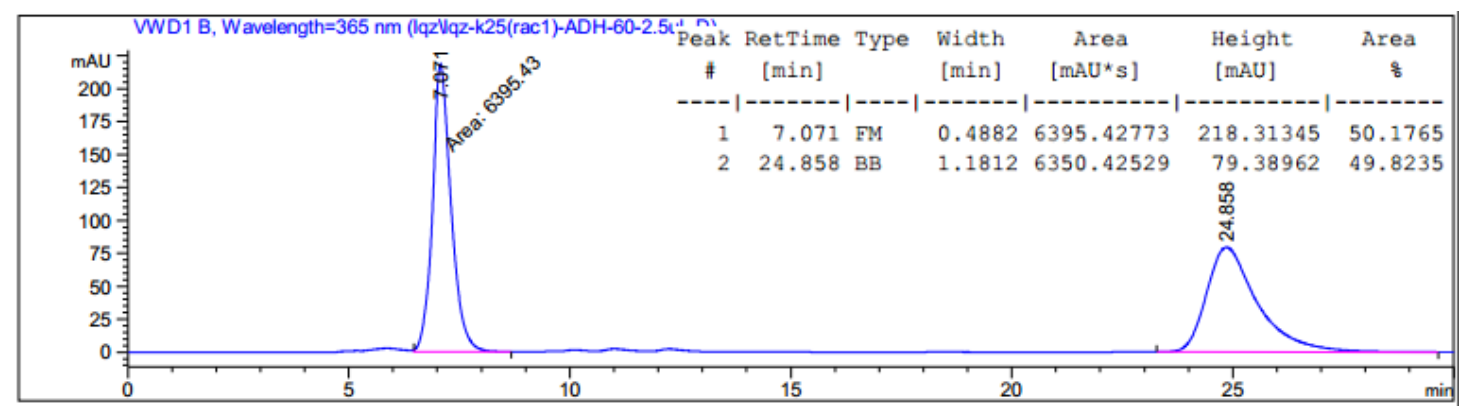

Chiral HPLC spectrum of 4 ua 

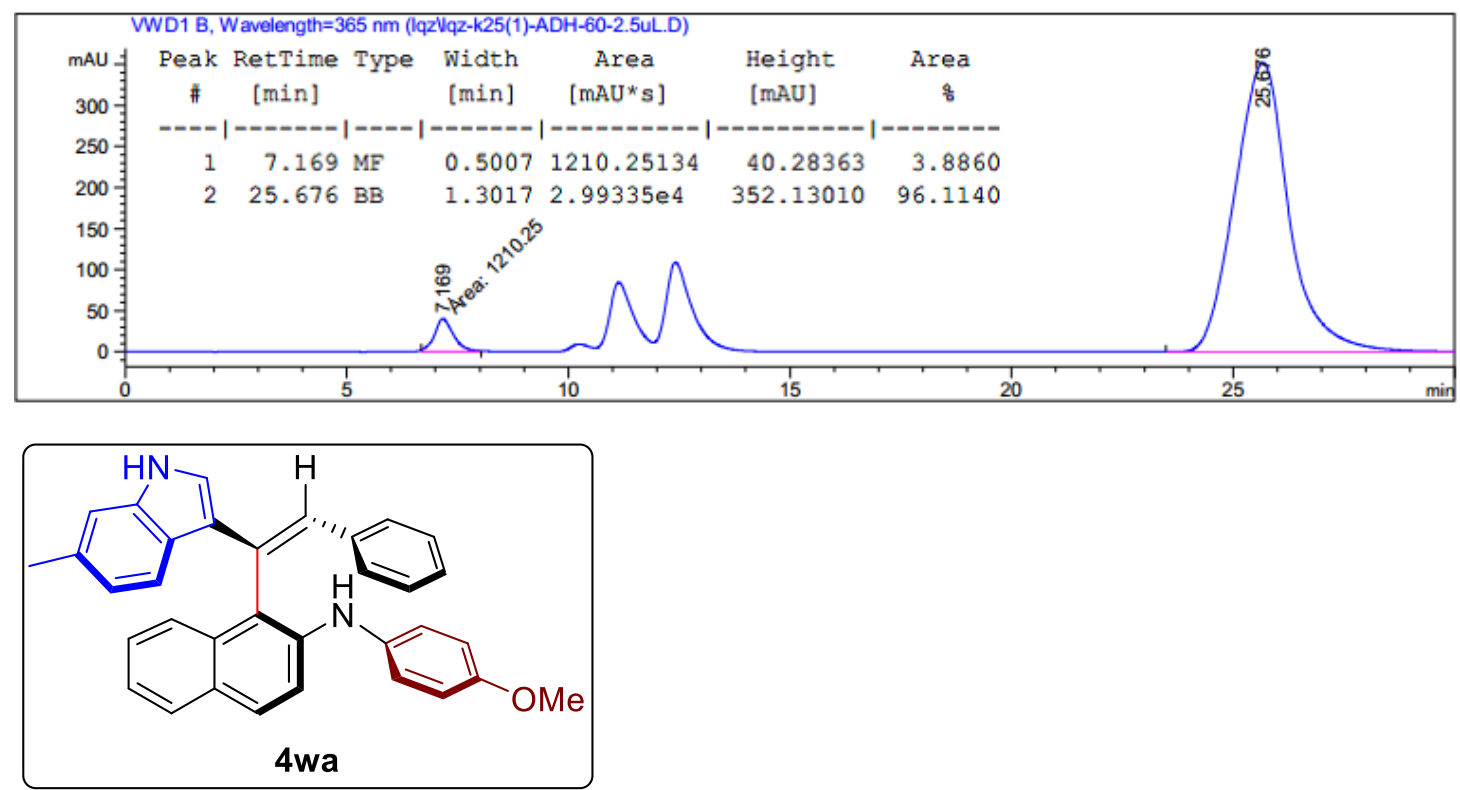

(aS)-(Z)-N-(4-methoxyphenyl)-1-(1-(6-methyl-1H-indol-3-yl)-2-phenylvinyl)naphthalen-2amine (4wa)

According to procedure B, 4wa was obtained as a yellowish solid, $44.2 \mathrm{mg}$ (92\% yield, $92 \%$ ee, Z:E > 20:1).

${ }^{1} \mathrm{H}$ NMR (400 MHz, CDCl $) \delta 8.10(\mathrm{~d}, J=8.2 \mathrm{~Hz}, 1 \mathrm{H}), 7.89(\mathrm{~s}, 1 \mathrm{H}), 7.79-7.75(\mathrm{~m}, 3 \mathrm{H}), 7.70(\mathrm{~s}, 1 \mathrm{H})$, 7.35 (d, $J=9.0 \mathrm{~Hz}, 1 \mathrm{H}), 7.28$ (s, 1H), $7.27-7.24$ (m, 2H), $7.17-7.13$ (m, 2H), 7.07 (s, 5H), $6.81-6.74$ (m, 4H), $6.66(\mathrm{~d}, J=2.7 \mathrm{~Hz}, 1 \mathrm{H}), 6.03(\mathrm{~s}, 1 \mathrm{H}), 3.77(\mathrm{~s}, 3 \mathrm{H}), 2.52(\mathrm{~s}, 3 \mathrm{H})$.

${ }^{13}$ C NMR (101 MHz, CDCl $) \delta$ 155.7, 139.9, 137.8, 137.4, 135.966.0, 133.3, 132.5, 131.5, 128.6, 128.5, $128.3,128.2,127.9,127.6,126.62,126.59,124.98,124.96,123.8,123.0,122.7,122.4,120.50,120.47$, $118.3,116.9,114.4,111.7,55.5,21.6$.

HRMS (ESI) calculated for $[\mathrm{M}+\mathrm{H}]^{+} \mathrm{C}_{34} \mathrm{H}_{29} \mathrm{~N}_{2} \mathrm{O}^{+}, \mathrm{m} / \mathrm{z}: 481.2280$, found: 481.2283 .

M.P. $88-90^{\circ} \mathrm{C}$.

$[\alpha]^{20} \mathrm{D}=-12^{\circ}\left(\mathrm{c}=0.2, \mathrm{CHCl}_{3}\right)$.

HPLC analysis: HPLC DAICEL CHIRALPAK OD-H, hexane/isopropyl alcohol $=90 / 10$, flow rate $=1$ $\mathrm{mL} / \mathrm{min}, \lambda=360 \mathrm{~nm}), \operatorname{tr}($ major $)=15.2 \mathrm{~min}, \operatorname{tr}($ minor $)=14.0 \mathrm{~min}$, ee $=92 \%$.

Chiral HPLC spectrum of racemic 4 wa

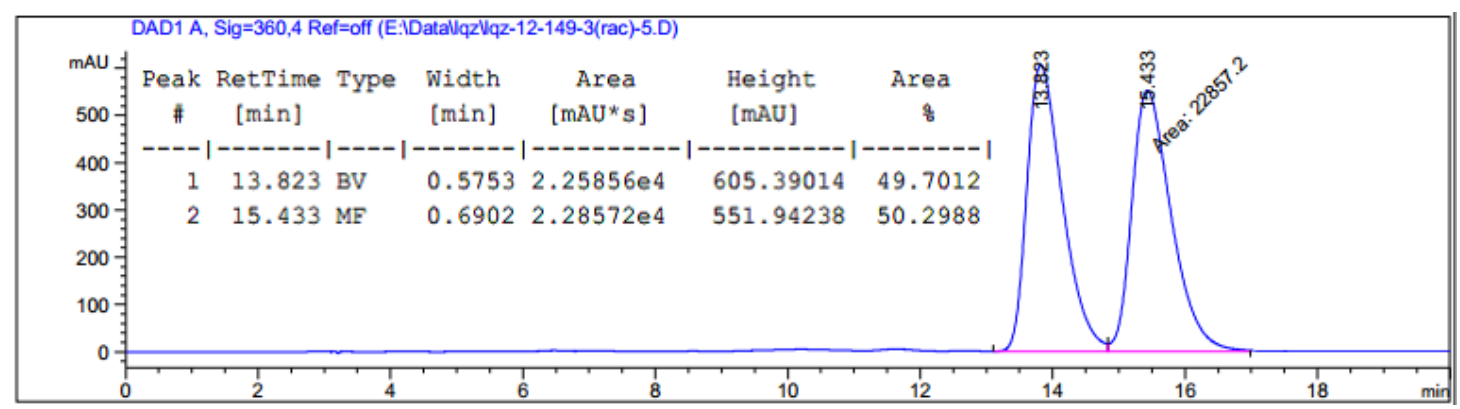

Chiral HPLC spectrum of $\mathbf{4 w a}$ 

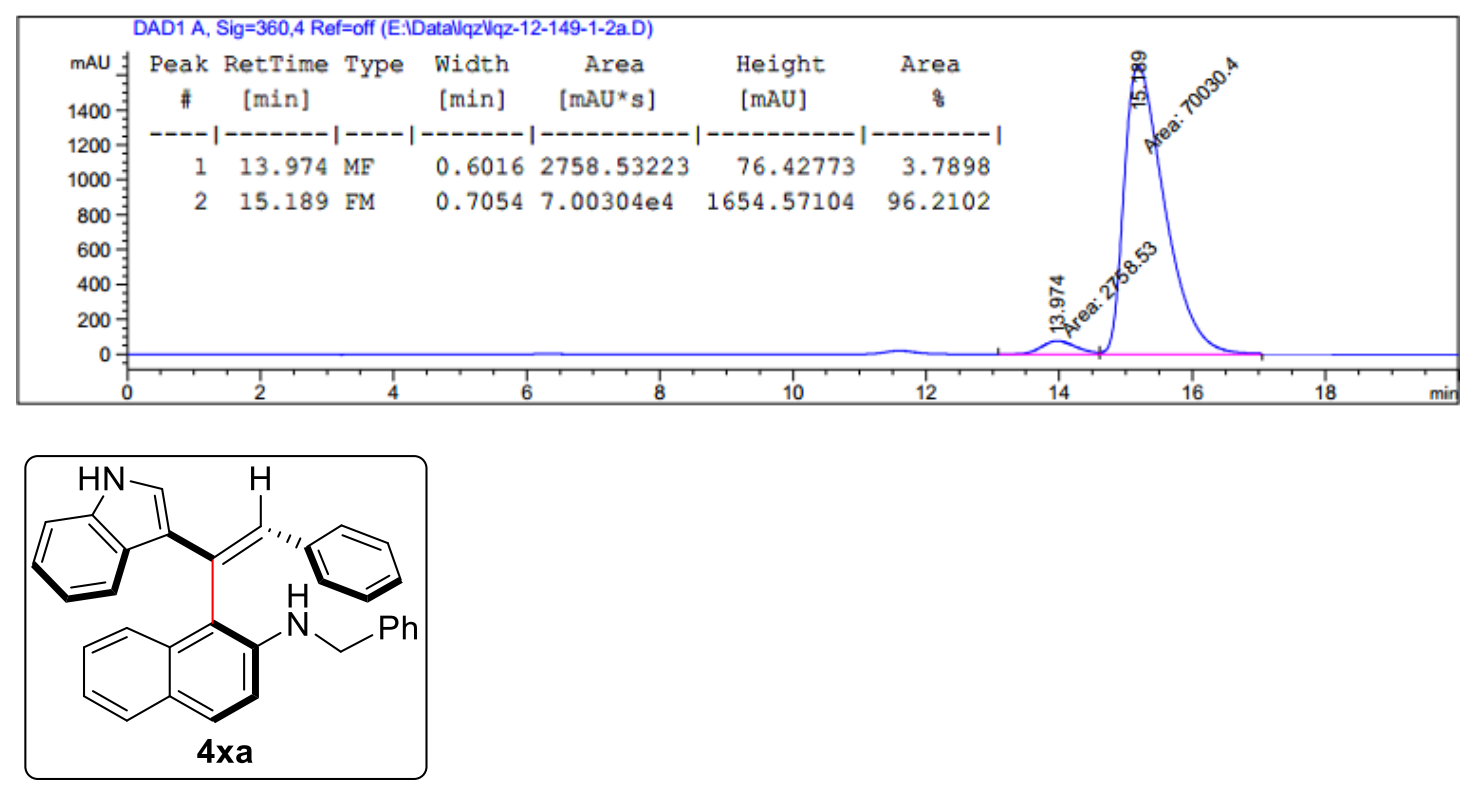

(aS)-(Z)-1-(1-(1H-indol-3-yl)-2-phenylvinyl)-N-benzylnaphthalen-2-amine (4xa)

According to procedure $\mathbf{B}, \mathbf{4} \mathbf{x a}$ was obtained as a yellow solid, $42.8 \mathrm{mg}$ (95\% yield, $93 \%$ ee, $\mathrm{Z}: \mathrm{E}=11: 1)$. ${ }^{1}$ H NMR (400 MHz, CDCl $) \delta 8.15(\mathrm{dd}, J=6.1,3.1 \mathrm{~Hz}, 1 \mathrm{H}), 7.93(\mathrm{~s}, 1 \mathrm{H}), 7.72-7.65(\mathrm{~m}, 4 \mathrm{H}), 7.36-$ $7.33(\mathrm{~m}, 1 \mathrm{H}), 7.27-7.24(\mathrm{~m}, 2 \mathrm{H}), 7.21-7.14(\mathrm{~m}, 2 \mathrm{H}), 7.11-7.00(\mathrm{~m}, 9 \mathrm{H}), 6.87-6.85(\mathrm{~m}, 2 \mathrm{H}), 6.59$ (d, $J=2.7 \mathrm{~Hz}, 1 \mathrm{H}), 4.81(\mathrm{t}, J=6.3 \mathrm{~Hz}, 1 \mathrm{H}), 4.35-4.24(\mathrm{~m}, 2 \mathrm{H})$.

${ }^{13} \mathbf{C}$ NMR (101 MHz, CDCl $) \delta 141.9,139.7,137.5,137.3,133.1,131.4,128.9,128.4,128.3,128.2$, $127.9,127.9,127.4,126.9,126.8,126.64,126.58,125.6,125.3,124.5,122.5,121.9,120.9,120.7,118.5$, $118.1,114.1,111.7,47.8$.

HRMS (ESI) calculated for $[\mathrm{M}+\mathrm{H}]^{+} \mathrm{C}_{33} \mathrm{H}_{27} \mathrm{~N}_{2}{ }^{+}, \mathrm{m} / \mathrm{z}: 451.2174$, found: 451.2171 .

M.P. $70-72^{\circ} \mathrm{C}$.

$[\alpha]^{20}{ }_{\mathrm{D}}=169^{\circ}\left(\mathrm{c}=0.2, \mathrm{CHCl}_{3}\right)$.

HPLC analysis: HPLC DAICEL CHIRALPAK IB, hexane/isopropyl alcohol $=90 / 10$, flow rate $=0.5$ $\mathrm{mL} / \mathrm{min}, \lambda=360 \mathrm{~nm}), \mathrm{tR}($ major $)=20.7 \mathrm{~min}, \mathrm{tR}($ minor $)=22.5 \mathrm{~min}, \mathrm{ee}=93 \%$.

Chiral HPLC spectrum of racemic $4 x \boldsymbol{a}$

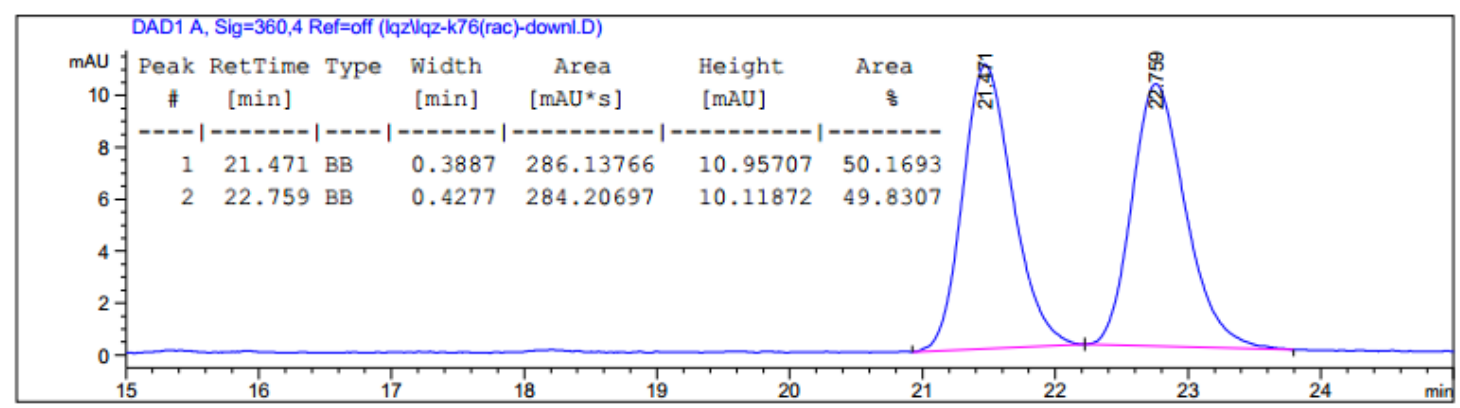

Chiral HPLC spectrum of $4 x a$ 

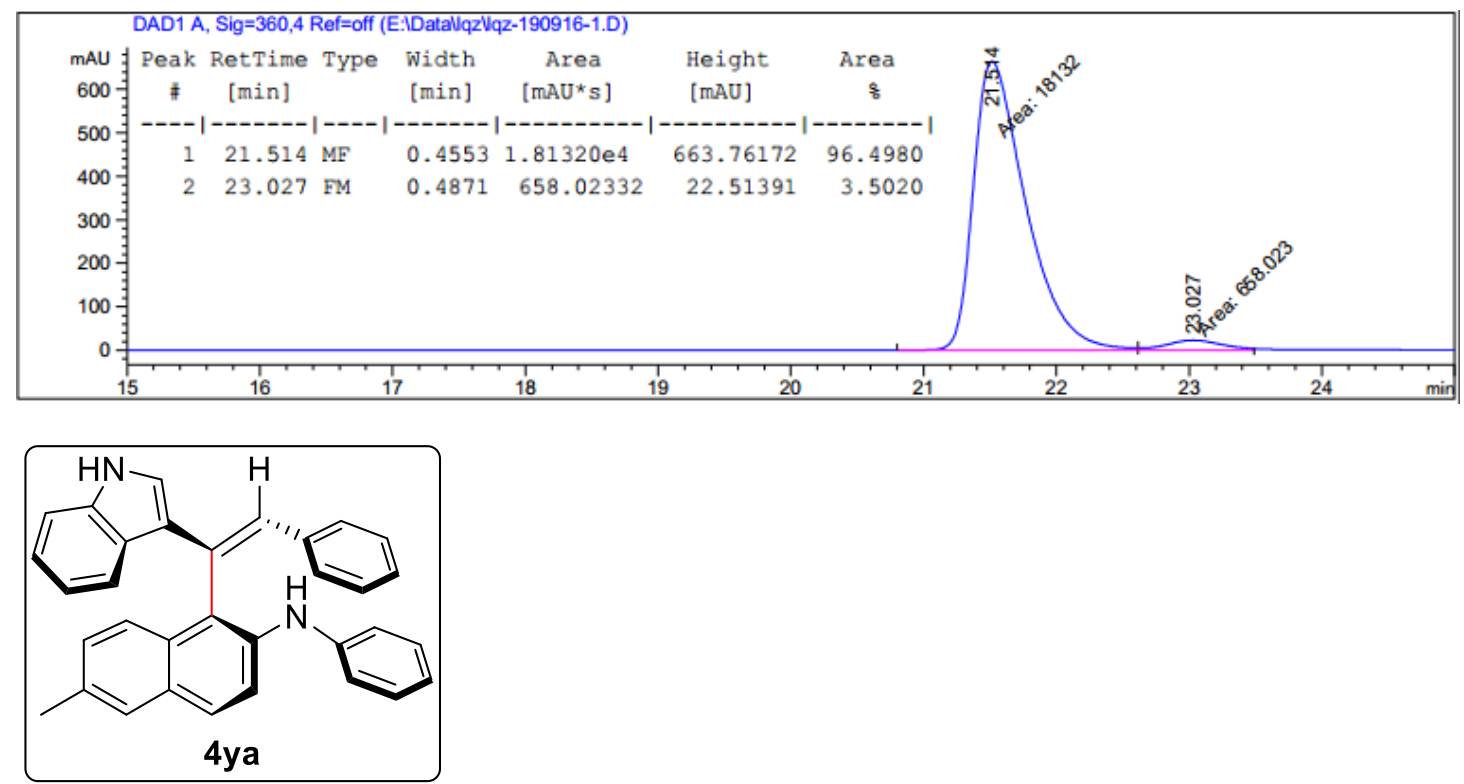

(aS)-(Z)-1-(1-(1H-indol-3-yl)-2-phenylvinyl)-6-methyl-N-phenylnaphthalen-2-amine (4ya)

According to procedure $\mathbf{B}, \mathbf{4 y a}$ was obtained as a yellow foam, $41.0 \mathrm{mg}$ (91\% yield, $91 \% \mathrm{ee}, \mathrm{Z}: \mathrm{E}=15: 1)$. ${ }^{1}$ H NMR (400 MHz, CDCl $) \delta 8.19-8.17(\mathrm{~m}, 1 \mathrm{H}), 7.94(\mathrm{~s}, 1 \mathrm{H}), 7.71-7.65(\mathrm{~m}, 3 \mathrm{H}), 7.55-7.52(\mathrm{~m}$, $2 \mathrm{H}), 7.35-7.32(\mathrm{~m}, 1 \mathrm{H}), 7.28-7.23(\mathrm{~m}, 2 \mathrm{H}), 7.13-7.06(\mathrm{~m}, 3 \mathrm{H}), 7.03-6.98(\mathrm{~m}, 5 \mathrm{H}), 6.85-6.81(\mathrm{~m}$, $3 \mathrm{H}), 6.68(\mathrm{~d}, J=2.7 \mathrm{~Hz}, 1 \mathrm{H}), 6.08(\mathrm{~s}, 1 \mathrm{H}), 2.42(\mathrm{~s}, 3 \mathrm{H})$.

${ }^{13} \mathbf{C}$ NMR (101 MHz, CDCl $) \delta 143.4,137.28,137.26,137.0,132.8,131.39,131.36,129.6,129.1,128.9$, $128.24,128.22,127.9,127.7,127.1,126.7,125.4,125.1,123.1,122.6,121.2,120.8,120.7,118.8,118.60$ 118.57, 111.7, 21.4 .

HRMS (ESI) calculated for $[\mathrm{M}+\mathrm{H}]^{+} \mathrm{C}_{33} \mathrm{H}_{27} \mathrm{~N}_{2}{ }^{+}, \mathrm{m} / \mathrm{z}: 451.2174$, found: 451.2171 .

M.P. $73-75^{\circ} \mathrm{C}$.

$[\alpha]^{20}{ }_{\mathrm{D}}=-22^{\circ}\left(\mathrm{c}=0.2, \mathrm{CHCl}_{3}\right)$.

HPLC analysis: HPLC DAICEL CHIRALPAK AD-H, hexane/isopropyl alcohol $=70 / 30$, flow rate $=1$ $\mathrm{mL} / \mathrm{min}, \lambda=254 \mathrm{~nm}$ ), $\mathrm{tR}$ (major) $=9.5 \mathrm{~min}, \mathrm{tr}($ minor $)=5.8 \mathrm{~min}, \mathrm{ee}=91 \%$.

Chiral HPLC spectrum of racemic $4 y \boldsymbol{a}$

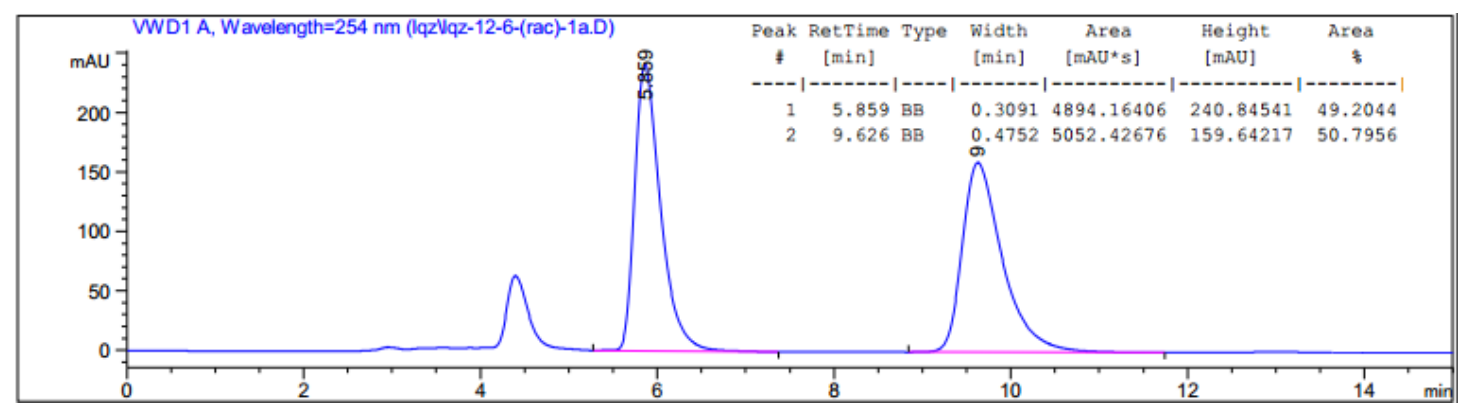

Chiral HPLC spectrum of $4 y a$ 

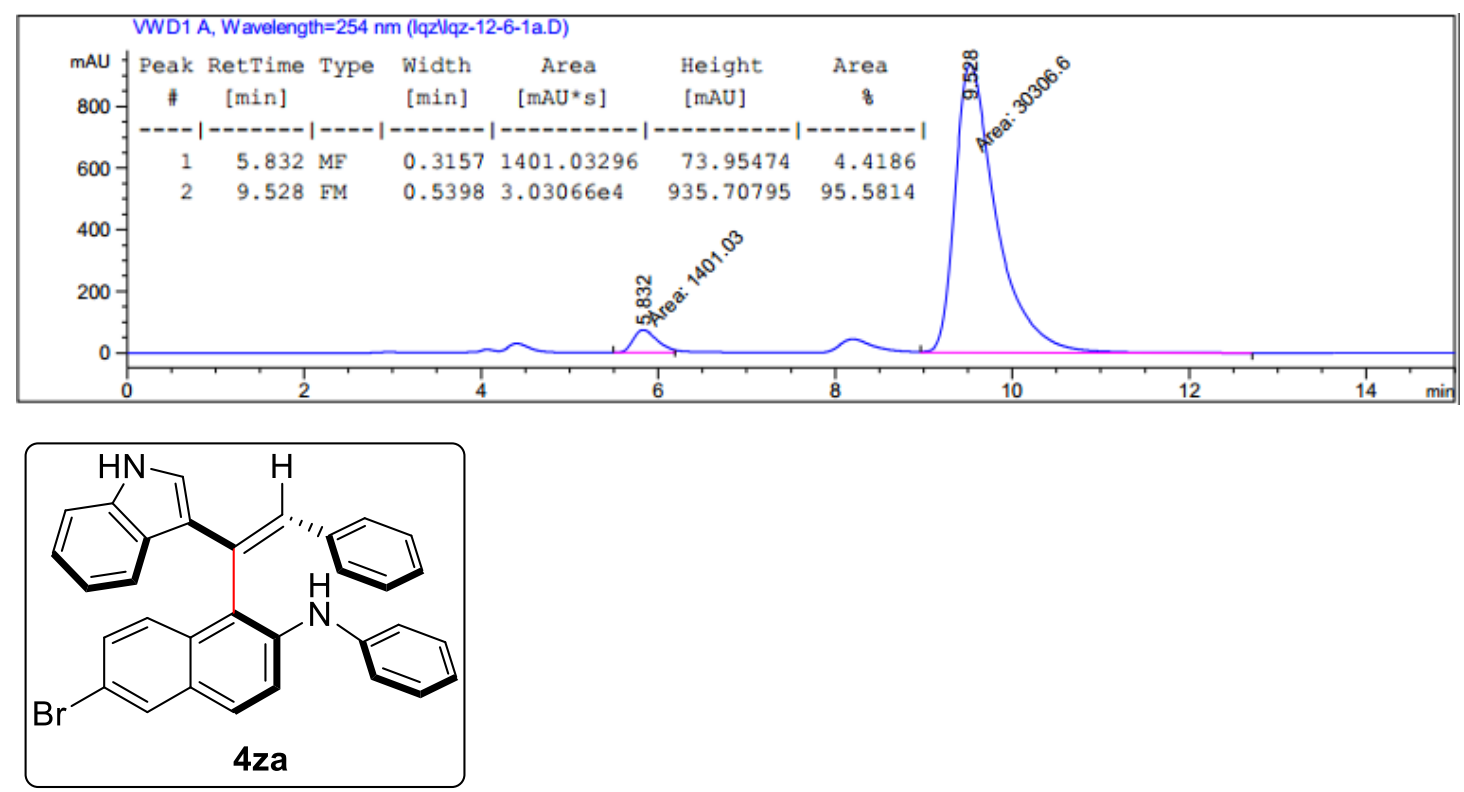

(aS)-(Z)-1-(1-(1H-indol-3-yl)-2-phenylvinyl)-6-bromo-N-phenylnaphthalen-2-amine (4za)

According to procedure $\mathbf{B}$, 4za was obtained as a yellow foam, $20.0 \mathrm{mg}$ (39\% yield, $93 \%$ ee, $\mathrm{Z}: \mathrm{E}=7: 1$ ). ${ }^{1} \mathbf{H}$ NMR (400 MHz, CDCl $) \delta 8.15-8.13(\mathrm{~m}, 1 \mathrm{H}), 8.04(\mathrm{~s}, 1 \mathrm{H}), 7.91(\mathrm{~d}, J=2.1 \mathrm{~Hz}, 1 \mathrm{H}), 7.68-7.61$ (m, 3H), $7.55(\mathrm{~d}, J=9.0 \mathrm{~Hz}, 1 \mathrm{H}), 7.39-7.36(\mathrm{~m}, 1 \mathrm{H}), 7.30-7.25(\mathrm{~m}, 3 \mathrm{H}), 7.17-7.13(\mathrm{~m}, 2 \mathrm{H}), 7.05-$ $7.02(\mathrm{~m}, 3 \mathrm{H}), 7.01-6.98(\mathrm{~m}, 2 \mathrm{H}), 6.89(\mathrm{td}, J=7.4,1.2 \mathrm{~Hz}, 1 \mathrm{H}), 6.86-6.83(\mathrm{~m}, 2 \mathrm{H}), 6.70(\mathrm{~d}, J=2.7$ $\mathrm{Hz}, 1 \mathrm{H}), 6.17(\mathrm{~s}, 1 \mathrm{H})$.

${ }^{13} \mathbf{C}$ NMR (101 MHz, $\left.\mathbf{C D C l}_{3}\right) \delta 142.5,138.5,137.3,137.0,131.8,130.6,130.2,129.93,129.91,129.2$, $128.3,128.24,128.19,127.5,127.0,126.9,125.3,125.0,122.8,122.3,122.0,120.9,120.8,119.8,118.8$, $118.3,116.9,111.8$.

HRMS (ESI) calculated for $[\mathrm{M}+\mathrm{H}]^{+} \mathrm{C}_{32} \mathrm{H}_{24} \mathrm{BrN}_{2}{ }^{+}, \mathrm{m} / \mathrm{z}$ : 515.1123 , found: 515.1165 .

M.P. $80-82^{\circ} \mathrm{C}$.

$[\alpha]^{20}{ }_{\mathrm{D}}=7^{\circ}\left(\mathrm{c}=0.2, \mathrm{CHCl}_{3}\right)$.

HPLC analysis: HPLC DAICEL CHIRALPAK AD-H, hexane/isopropyl alcohol $=70 / 30$, flow rate $=1$ $\mathrm{mL} / \mathrm{min}, \lambda=254 \mathrm{~nm}$ ), $\mathrm{tR}$ (major) $=8.0 \mathrm{~min}, \mathrm{tR}($ minor $)=6.7 \mathrm{~min}$, ee $=93 \%$.

Chiral HPLC spectrum of racemic $4 z a$

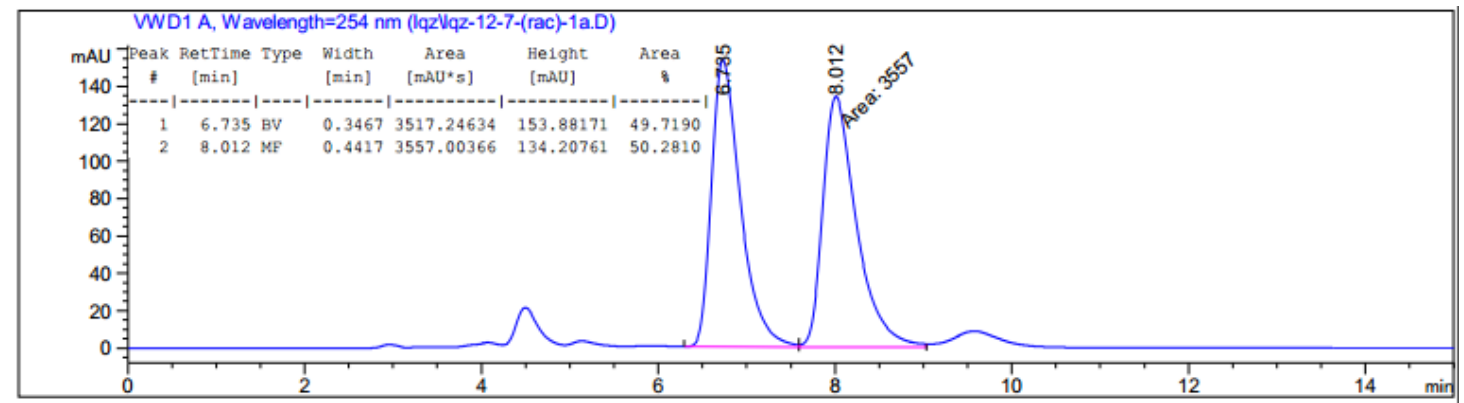

Chiral HPLC spectrum of $\mathbf{4 z a}$ 

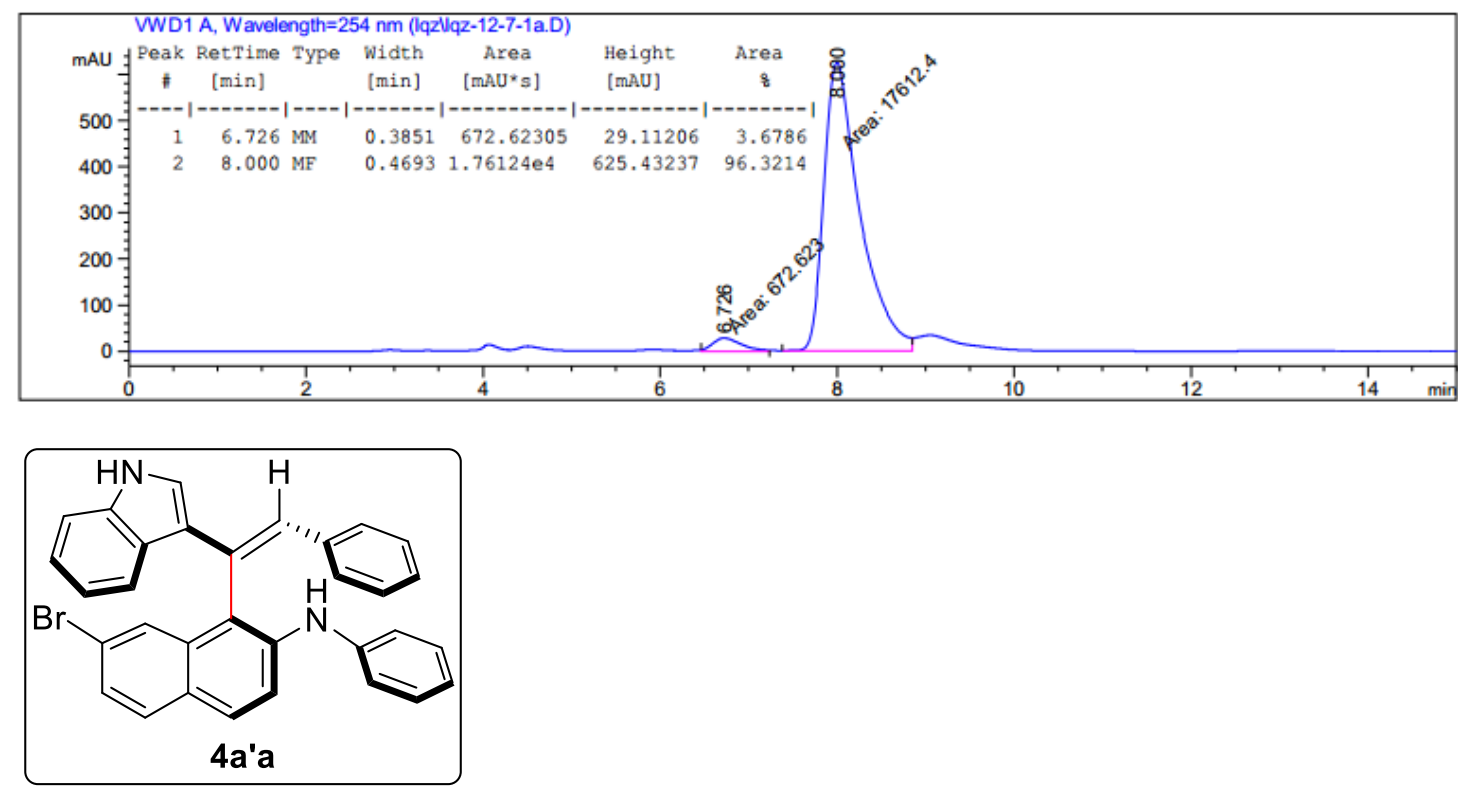

(aS)-(Z)-1-(1-(1H-indol-3-yl)-2-phenylvinyl)-7-bromo-N-phenylnaphthalen-2-amine (4a'a) According to procedure $\mathbf{B}, \mathbf{4 a} \mathbf{a}$ was obtained as a yellow foam, $26.7 \mathrm{mg}$ (52\% yield, $94 \%$ ee, $\mathrm{Z}: \mathrm{E}=$ 12:1)

${ }^{1}$ H NMR (400 MHz, CDCl $) \delta 8.14-8.12(\mathrm{~m}, 1 \mathrm{H}), 7.94(\mathrm{~s}, 1 \mathrm{H}), 7.90(\mathrm{~d}, J=1.9 \mathrm{~Hz}, 1 \mathrm{H}), 7.70(\mathrm{~d}, J=$ $8.2 \mathrm{~Hz}, 2 \mathrm{H}), 7.59(\mathrm{~d}, J=8.7 \mathrm{~Hz}, 1 \mathrm{H}), 7.52(\mathrm{~d}, J=9.0 \mathrm{~Hz}, 1 \mathrm{H}), 7.33-7.22(\mathrm{~m}, 4 \mathrm{H}), 7.15-7.11(\mathrm{~m}, 2 \mathrm{H})$, $7.06-6.97(\mathrm{~m}, 5 \mathrm{H}), 6.90-6.86(\mathrm{~m}, 1 \mathrm{H}), 6.85-6.83(\mathrm{~m}, 2 \mathrm{H}), 6.66(\mathrm{~d}, J=2.7 \mathrm{~Hz}, 1 \mathrm{H}), 6.18(\mathrm{~s}, 1 \mathrm{H})$.

${ }^{13} \mathbf{C}$ NMR (101 MHz, CDCl $) \delta$ ) 142.4, 139.3, 137.4, 137.1, 134.6, 130.5, 129.7, 129.2, 128.5, 128.31, $128.28,127.4,127.0,126.9,126.6,125.4,125.1,122.8,122.3,121.3,121.2,120.88,120.86,120.1,118.0$, $117.9,111.8$.

HRMS (ESI) calculated for $[\mathrm{M}+\mathrm{H}]^{+} \mathrm{C}_{32} \mathrm{H}_{24} \mathrm{BrN}_{2}{ }^{+}, \mathrm{m} / \mathrm{z}$ : 515.1123 , found: 515.1142 .

M.P. $70-72{ }^{\circ} \mathrm{C}$.

$[\alpha]^{20}{ }_{\mathrm{D}}=33^{\circ}\left(\mathrm{c}=0.2, \mathrm{CHCl}_{3}\right)$.

HPLC analysis: HPLC DAICEL CHIRALPAK AD-H, hexane/isopropyl alcohol $=70 / 30$, flow rate $=1$ $\mathrm{mL} / \mathrm{min}, \lambda=254 \mathrm{~nm}$ ), $\mathrm{tR}$ (major) $=6.5 \mathrm{~min}, \mathrm{tR}($ minor $)=4.8 \mathrm{~min}$, ee $=94 \%$.

Chiral HPLC spectrum of racemic $4 \boldsymbol{a}^{\prime} \boldsymbol{a}$

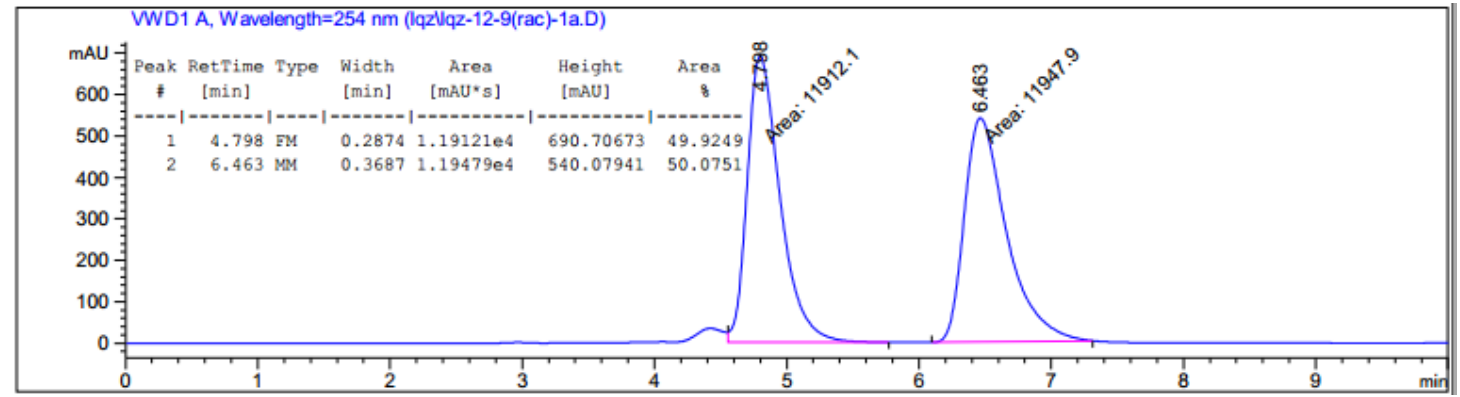

Chiral HPLC spectrum of $\mathbf{4 a}$ 'a 

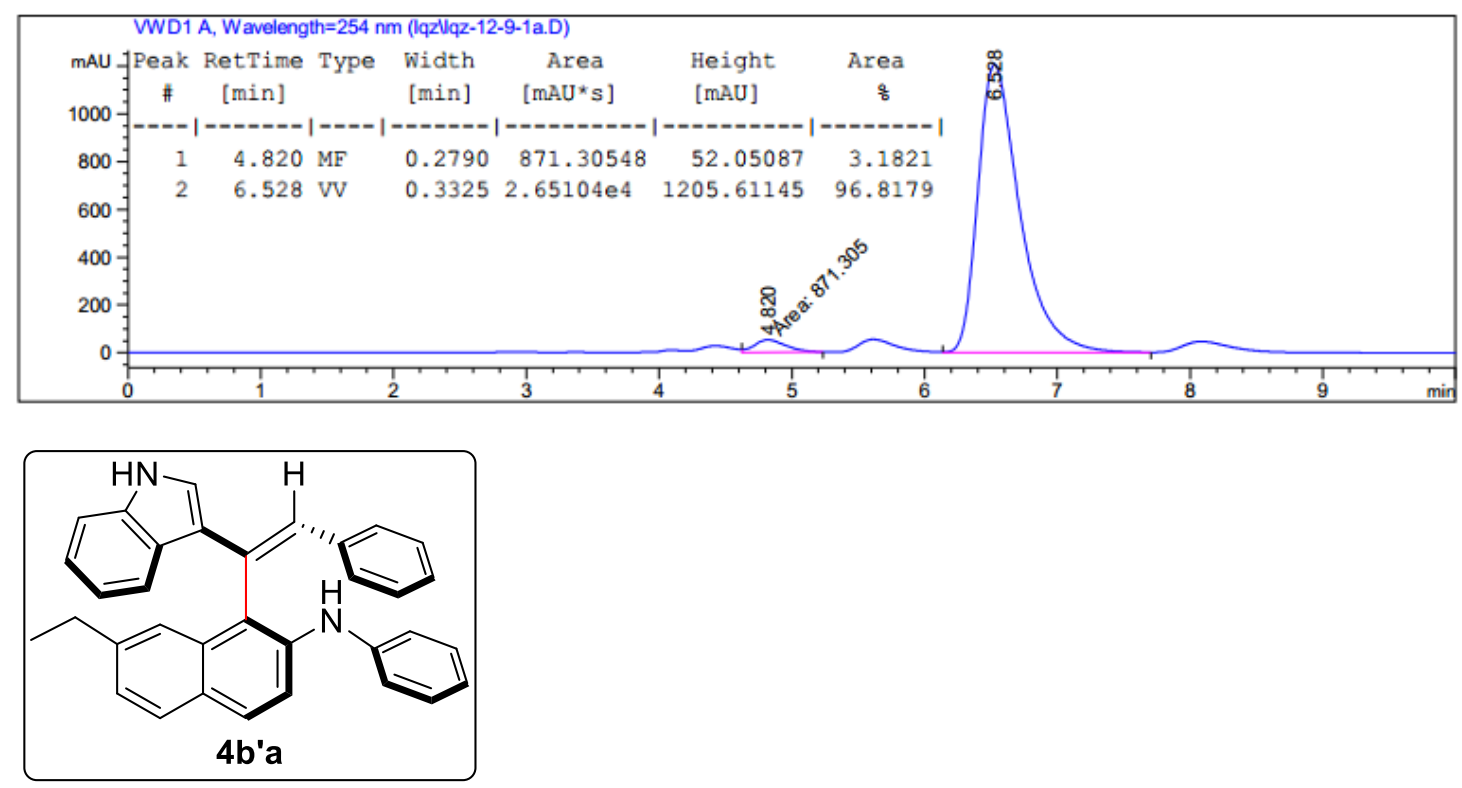

(aS)-(Z)-1-(1-(1H-indol-3-yl)-2-phenylvinyl)-7-ethyl-N-phenylnaphthalen-2-amine (4b'a)

According to procedure B, $\mathbf{4 b}$ 'a was obtained as a yellow solid, $43.2 \mathrm{mg}$ (93\% yield, $94 \%$ ee, Z:E = $17: 1)$.

${ }^{1}$ H NMR (400 MHz, CDCl $)$ ) $\delta 8.19-8.17(\mathrm{~m}, 1 \mathrm{H}), 7.8(\mathrm{~s}, 1 \mathrm{H}), 7.73-7.67(\mathrm{~m}, 3 \mathrm{H}), 7.55(\mathrm{~s}, 1 \mathrm{H}), 7.48$ (d, $J=8.9 \mathrm{~Hz}, 1 \mathrm{H}), 7.31-7.21(\mathrm{~m}, 3 \mathrm{H}), 7.14-7.09$ (m, 3H), $7.01-6.99$ (m, 5H), $6.84-6.81(\mathrm{~m}, 3 \mathrm{H})$, $6.66(\mathrm{~d}, J=2.7 \mathrm{~Hz}, 1 \mathrm{H}), 6.10(\mathrm{~s}, 1 \mathrm{H}), 2.55(\mathrm{q}, J=7.5 \mathrm{~Hz}, 2 \mathrm{H}), 1.06(\mathrm{t}, J=7.6 \mathrm{~Hz}, 3 \mathrm{H})$.

${ }^{13} \mathbf{C}$ NMR (101 MHz, $\left.\mathbf{C D C l}_{3}\right) \delta 143.2,142.6,137.8,137.4,137.3,133.4,131.4,129.1,128.3,128.2$, $128.1,128.0,127.9,127.8,126.6,125.4,125.2,124.5,123.0,122.60,122.56,121.4,120.8,120.7,119.1$, $118.5,117.4,111.7,29.2,15.5$.

HRMS (ESI) calculated for $[\mathrm{M}+\mathrm{H}]^{+} \mathrm{C}_{34} \mathrm{H}_{29} \mathrm{~N}_{2}{ }^{+}, \mathrm{m} / \mathrm{z}$ : 465.2331 , found: 465.2328 .

M.P. $60-63{ }^{\circ} \mathrm{C}$.

$[\alpha]^{20}{ }_{\mathrm{D}}=-5^{\circ}\left(\mathrm{c}=0.2, \mathrm{CHCl}_{3}\right)$.

HPLC analysis: HPLC DAICEL CHIRALPAK AD-H, hexane/isopropyl alcohol $=80 / 20$, flow rate $=1$ $\mathrm{mL} / \mathrm{min}, \lambda=360 \mathrm{~nm}$ ), $\mathrm{tR}$ (major) $=10.1 \mathrm{~min}, \mathrm{tR}(\operatorname{minor})=5.3 \mathrm{~min}$, ee $=94 \%$.

Chiral HPLC spectrum of racemic $\mathbf{4} \boldsymbol{b}$ 'a

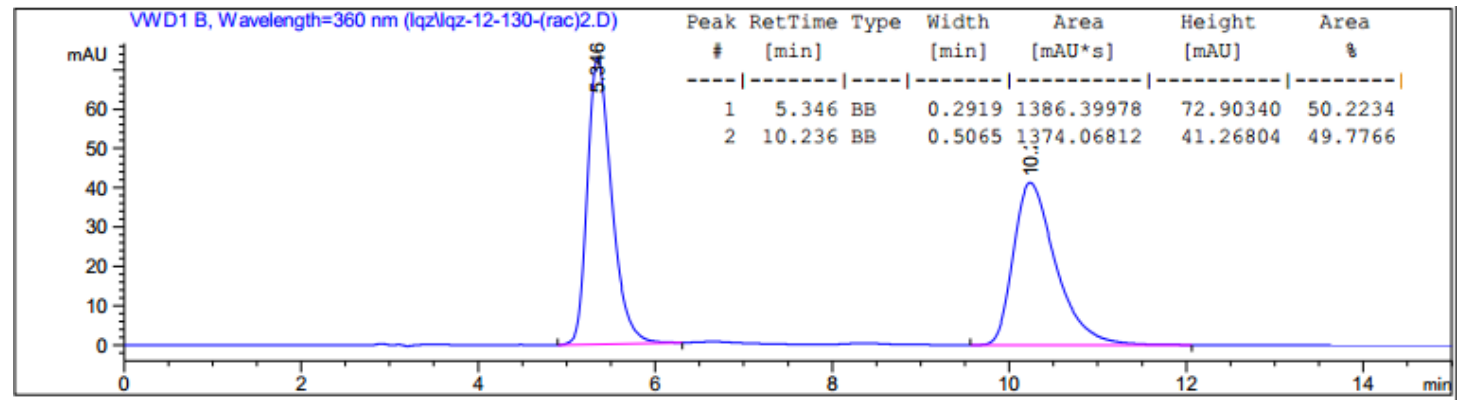

Chiral HPLC spectrum of $\mathbf{4} \boldsymbol{b}^{\prime} \boldsymbol{a}$ 

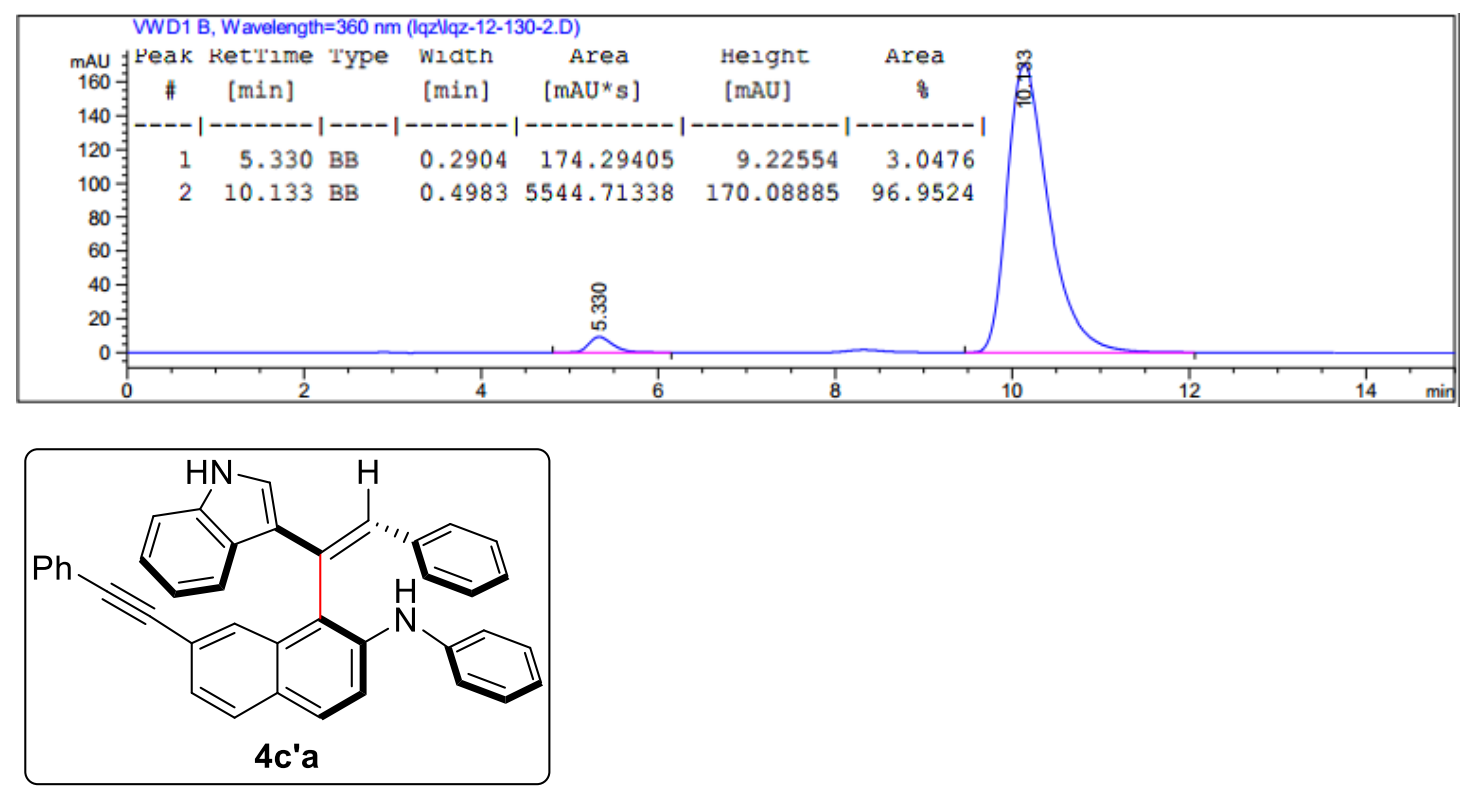

(aS)-(Z)-1-(1-(1H-indol-3-yl)-2-phenylvinyl)-N-phenyl-7-(phenylethynyl)naphthalen-2-amine (4c'a)

According to procedure B, 4c'a was obtained as a yellow foam, $11.8 \mathrm{mg}$ (22\% yield, 90\% ee, Z:E = 5:1). ${ }^{1}$ H NMR (400 MHz, CDCl $) \delta 8.14-8.12(\mathrm{~m}, 1 \mathrm{H}), 8.05(\mathrm{~d}, J=2.6 \mathrm{~Hz}, 1 \mathrm{H}), 7.93(\mathrm{~d}, J=1.9 \mathrm{~Hz}, 1 \mathrm{H})$, $7.77(\mathrm{~d}, J=9.0 \mathrm{~Hz}, 1 \mathrm{H}), 7.70(\mathrm{~s}, 1 \mathrm{H}), 7.66(\mathrm{~d}, J=8.9 \mathrm{~Hz}, 1 \mathrm{H}), 7.58(\mathrm{~d}, J=8.9 \mathrm{~Hz}, 1 \mathrm{H}), 7.49-7.46(\mathrm{~m}$, 2H), $7.39-7.26(\mathrm{~m}, 10 \mathrm{H}), 7.04-7.03(\mathrm{~m}, 3 \mathrm{H}), 6.97-6.95(\mathrm{~m}, 2 \mathrm{H}), 6.80-6.78(\mathrm{~m}, 2 \mathrm{H}), 6.70(\mathrm{~d}, J=$ $2.7 \mathrm{~Hz}, 1 \mathrm{H}), 6.24(\mathrm{~s}, 1 \mathrm{H})$.

${ }^{13}$ C NMR (101 MHz, $\left.\mathbf{C D C l}_{3}\right) \delta 142.8,137.9,137.3,136.9,134.5,132.7,131.4,130.3,129.7,129.2$, $128.5,128.32,128.30,128.26,128.24,128.22$, 127.9, 127.2, 127.1, 127.0, 125.2, 125.0, 123.6, 122.8, $121.4,120.9,120.8,118.8,118.1,118.0,115.7,111.8,89.7,88.4$.

HRMS (ESI) calculated for $[\mathrm{M}+\mathrm{H}]^{+} \mathrm{C}_{40} \mathrm{H}_{29} \mathrm{~N}_{2}{ }^{+}, \mathrm{m} / \mathrm{z}$ : 537.2331, found: 537.2321.

M.P. $68-70^{\circ} \mathrm{C}$.

$[\alpha]^{20}{ }_{\mathrm{D}}=-55^{\circ}\left(\mathrm{c}=0.2, \mathrm{CHCl}_{3}\right)$.

HPLC analysis: HPLC DAICEL CHIRALPAK AD-H, hexane/isopropyl alcohol = 70/30, flow rate = 1 $\mathrm{mL} / \mathrm{min}, \lambda=365 \mathrm{~nm})$, $\mathrm{tR}$ (major) $=21.5 \mathrm{~min}, \mathrm{tR}($ minor $)=6.4 \mathrm{~min}$, ee $=90 \%$.

Chiral HPLC spectrum of racemic $4 c^{\prime}$ a

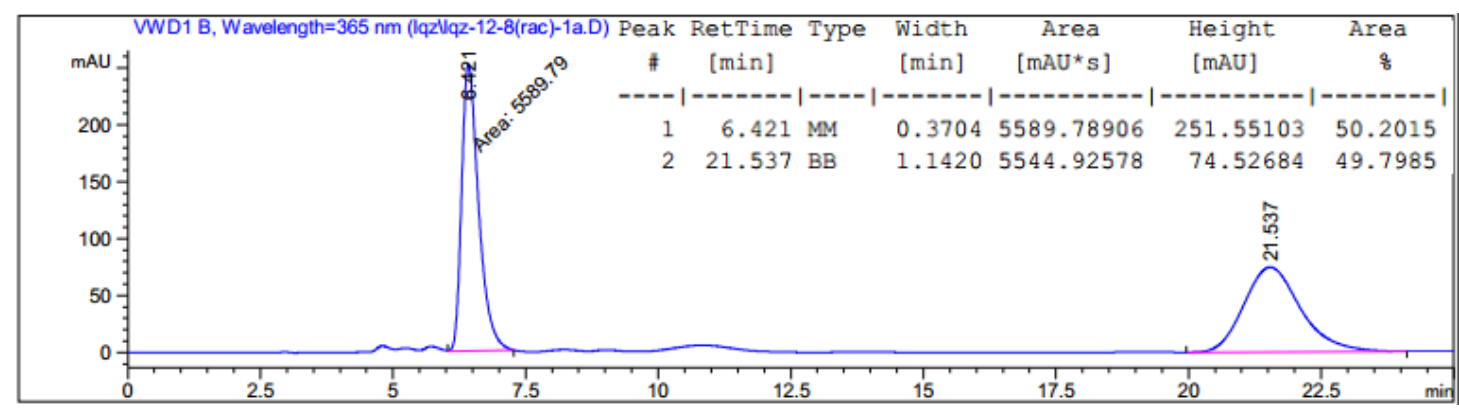

Chiral HPLC spectrum of $4 c^{\prime} \boldsymbol{a}$ 


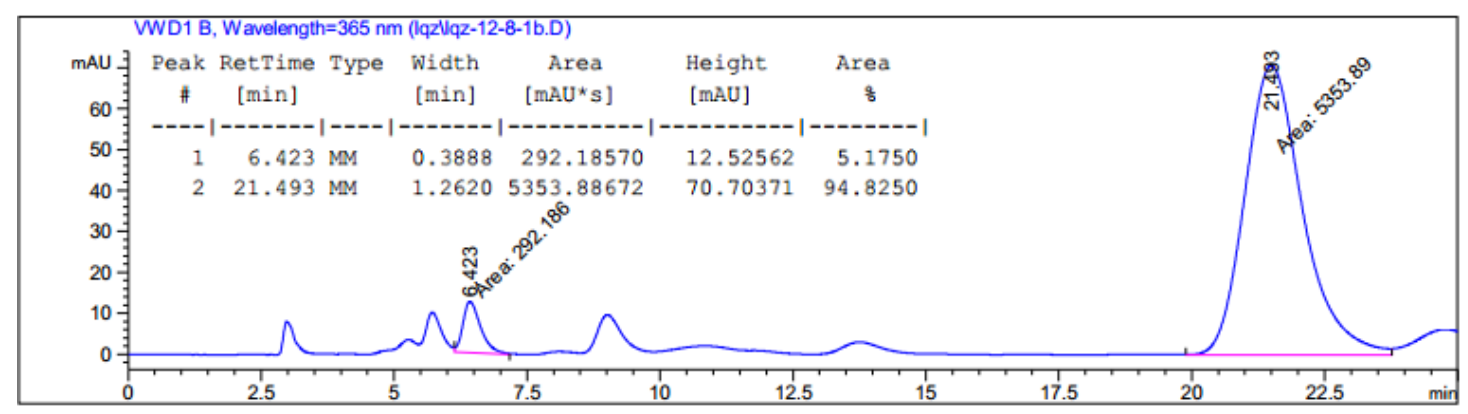


Table S2. Optimization of Reaction Conditions ${ }^{a}$<smiles>C(#Cc1c(Nc2ccccc2)ccc2ccccc12)c1ccccc1</smiles>

$1 \mathbf{a}$<smiles>O=c1cc(O)c2ccccc2o1</smiles>

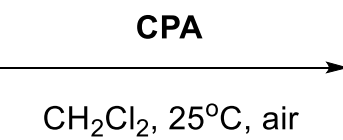

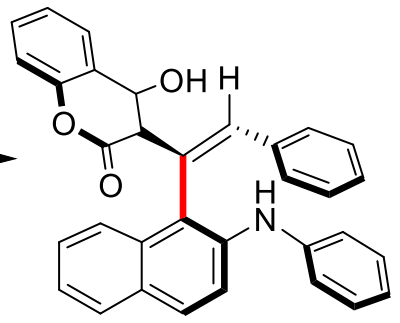

$6 a a$

\begin{tabular}{|c|c|c|c|c|c|c|}
\hline Entry & Cat. & Solvent & Additive & yield & ee & $\mathbf{E} / \mathbf{Z}$ \\
\hline 1 & CPA2 & $\mathrm{CH}_{2} \mathrm{Cl}_{2}$ & none & $34.5 \%$ & $-38 \%$ & $>20: 1$ \\
\hline 3 & CPA3 & $\mathrm{CH}_{2} \mathrm{Cl}_{2}$ & none & $28 \%$ & $-33 \%$ & $>20: 1$ \\
\hline 4 & CPA4 & $\mathrm{CH}_{2} \mathrm{Cl}_{2}$ & none & $8 \%$ & $-48 \%$ & $>20: 1$ \\
\hline 6 & CPA6 & $\mathrm{CH}_{2} \mathrm{Cl}_{2}$ & none & $12 \%$ & $-66 \%$ & $>20: 1$ \\
\hline 5 & CPA7 & $\mathrm{CH}_{2} \mathrm{Cl}_{2}$ & none & $52 \%$ & $-90 \%$ & $>20: 1$ \\
\hline 2 & CPA8 & $\mathrm{CH}_{2} \mathrm{Cl}_{2}$ & none & $5 \%$ & $-18 \%$ & $>20: 1$ \\
\hline 7 & CPA9 & $\mathrm{CH}_{2} \mathrm{Cl}_{2}$ & none & $45 \%$ & $-44 \%$ & $>20: 1$ \\
\hline 8 & CPA7 & DCE & none & 34 & $-86 \%$ & $>20: 1$ \\
\hline 9 & CPA7 & $\mathrm{CHCl}_{3}$ & none & $37 \%$ & $-84 \%$ & $>20: 1$ \\
\hline 10 & CPA7 & Toluene & none & $33 \%$ & $-87 \%$ & $>20: 1$ \\
\hline 11 & CPA7 & THF & none & \multicolumn{3}{|c|}{ Trace } \\
\hline 12 & CPA7 & $\mathrm{Et}_{2} \mathrm{O}$ & none & $<10 \%$ & $-95 \%$ & $>20: 1$ \\
\hline 13 & CPA7 & $\mathrm{MeCN}$ & none & $<10 \%$ & $-83 \%$ & $>20: 1$ \\
\hline 14 & CPA7 & $\mathrm{CH}_{2} \mathrm{Cl}_{2}$ & $3 \AA \mathrm{MS}($ 活化) $=50 \mathrm{mg}$ & $7.5 \%$ & $-87 \%$ & $>20: 1$ \\
\hline 15 & CPA7 & $\mathrm{CH}_{2} \mathrm{Cl}_{2}$ & $4 \AA \mathrm{MS}($ 活化 $)=50 \mathrm{mg}$ & $8.3 \%$ & $-88 \%$ & $>20: 1$ \\
\hline 16 & CPA7 & $\mathrm{CH}_{2} \mathrm{Cl}_{2}$ & $5 \AA \mathrm{MS}($ 活化 $)=50 \mathrm{mg}$ & $66.5 \%$ & $-92.5 \%$ & $>20: 1$ \\
\hline 17 & CPA7 & $\mathrm{CH}_{2} \mathrm{Cl}_{2}$ & $\mathrm{MgSO}_{4}=50 \mathrm{mg}$ & $73 \%$ & $-92 \%$ & $>20: 1$ \\
\hline $18^{b}$ & CPA7 & $\mathrm{CH}_{2} \mathrm{Cl}_{2}$ & $\mathrm{MgSO}_{4}=50 \mathrm{mg}$ & $84 \%$ & $-92 \%$ & $>20: 1$ \\
\hline $19^{c}$ & CPA7 & $\mathrm{CH}_{2} \mathrm{Cl}_{2}$ & $\mathrm{MgSO}_{4}=50 \mathrm{mg}$ & $92 \%$ & $-92 \%$ & $>20: 1$ \\
\hline $20^{c d}$ & CPA7 & $\mathrm{CH}_{2} \mathrm{Cl}_{2}$ & $\mathrm{MgSO}_{4}=50 \mathrm{mg}$ & $90 \%$ & $-94 \%$ & $>20: 1$ \\
\hline
\end{tabular}

${ }^{a}$ Reaction conditions: $1(0.05 \mathrm{mmol}), \mathbf{5}(0.1 \mathrm{mmol})$, catalyst $(0.005 \mathrm{mmol})$ in Toluene $(0.5 \mathrm{~mL})$ at $25^{\circ} \mathrm{C}$ for $24 \mathrm{~h}$ to $48 \mathrm{~h}$. Isolated yields were given. The ee values were determined by HPLC analysis using a chiral stationary phase. The Z/E values were determined by NMR analysis. ${ }^{b} \mathbf{1}(0.075 \mathrm{mmol}), \mathbf{5}(0.05 \mathrm{mmol}) .{ }^{c} \mathbf{1}(0.1 \mathrm{mmol}), \mathbf{5}(0.05 \mathrm{mmol}) .{ }^{d}$ temperature $=20^{\circ} \mathrm{C}$.<smiles>[R]c1cc2ccccc2c(-c2c(OP(=O)(O)O)c([R])cc3ccccc23)c1[R]</smiles>

CPA2, $\mathrm{R}=\mathrm{C}_{6} \mathrm{H}_{5}$

CPA3, $\mathrm{R}=4-\mathrm{OMeC}_{6} \mathrm{H}_{4}$

CPA4, $\mathrm{R}=4-\mathrm{NO}_{2} \mathrm{C}_{6} \mathrm{H}_{4}$

CPA6, R=9-anthracenyl

CPA7, $\mathrm{R}=2,4,6-(\mathrm{Pr})_{3} \mathrm{C}_{6} \mathrm{H}_{2}$

CPA8, $\mathrm{R}=\mathrm{Si}\left(\mathrm{C}_{6} \mathrm{H}_{5}\right)_{3}$

CPA9, R=2-naphthalenyl

Procedure C (Experimental procedure for the synthesis of racemic product) 

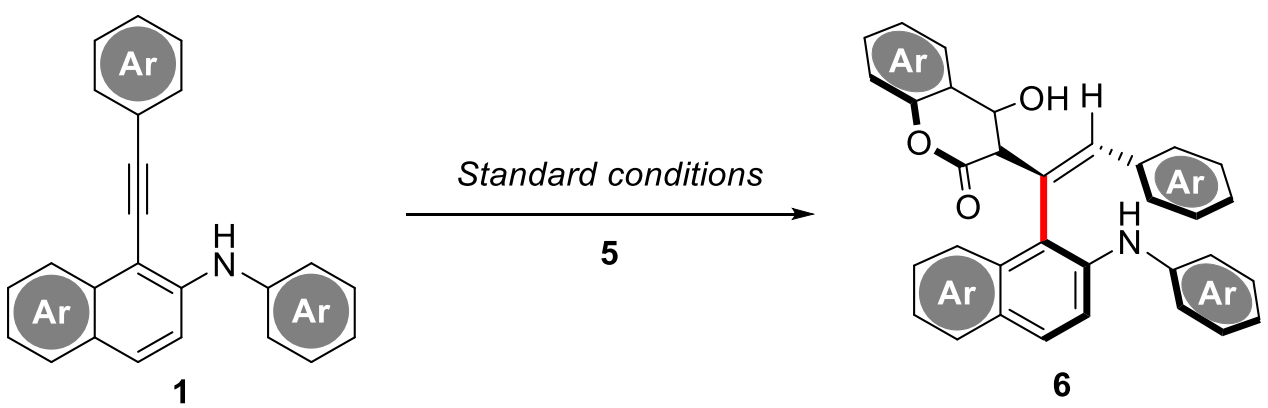

To a $10.0 \mathrm{~mL}$ reaction tube, $5(0.1 \mathrm{mmol}), \mathbf{1}(0.1 \mathrm{mmol}),(\mathrm{PhO})_{2} \mathrm{PO}_{2} \mathrm{H}(2.5 \mathrm{mg}, 0.01 \mathrm{mmol})$ and $1 \mathrm{~mL}$ DCM was added. The reaction mixture was stirred at ambient temperature and monitored by TLC. After completion of the reaction, the mixture was purified by preparative TLC on silica gel to yield the target molecular racemic product.

Procedure D (Experimental procedure for the synthesis of asymmetric product)
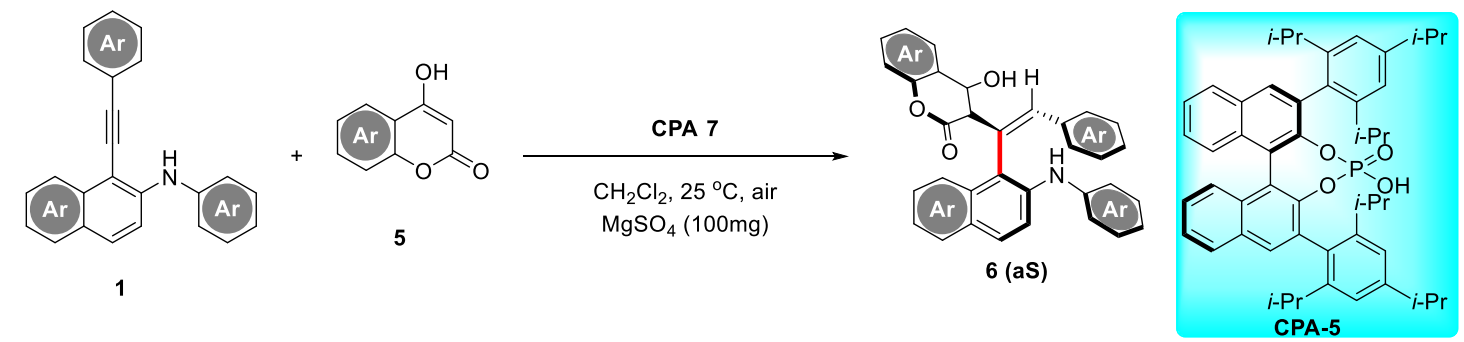

To a solution of 1 (0.2 mmol), CPA7 $(7.5 \mathrm{mg}, 0.010 \mathrm{mmol})$ and $\mathrm{MgSO}_{4}(100 \mathrm{mg})$ in $\mathrm{CH}_{2} \mathrm{Cl}_{2}(1 \mathrm{~mL})$, $1(0.1 \mathrm{mmol})$ was added in one portion at $25^{\circ} \mathrm{C}$ and monitored by TLC. After stirring for $48 \mathrm{~h}$, the mixture was directly purified through flash column chromatography on silica gel to give the pure product.

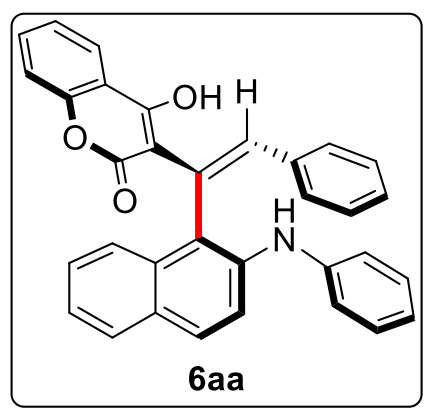

(aS)-(E)-4-hydroxy-3-(2-phenyl-1-(2-(phenylamino)naphthalen-1-yl)vinyl)-2H-chromen-2one (6aa)

According to procedure D, 6aa was obtained as a yellow solid, $43.3 \mathrm{mg}$ (90\% yield, 94\% ee).

${ }^{1}$ H NMR (400 MHz, CDCl $) \delta 10.54(\mathrm{dr}, 1 \mathrm{H}), 8.41(\mathrm{~d}, J=8.0 \mathrm{~Hz}, 1 \mathrm{H}), 7.78(\mathrm{~d}, J=8.1 \mathrm{~Hz}, 2 \mathrm{H}), 7.72$ $(\mathrm{d}, J=9.0 \mathrm{~Hz}, 1 \mathrm{H}), 7.55-7.51(\mathrm{~m}, 1 \mathrm{H}), 7.47(\mathrm{t}, J=7.8 \mathrm{~Hz}, 1 \mathrm{H}), 7.41-7.38(\mathrm{~m}, 2 \mathrm{H}), 7.35-7.27(\mathrm{~m}$, $3 \mathrm{H}), 7.19-7.10(\mathrm{~m}, 6 \mathrm{H}), 7.01(\mathrm{~d}, J=7.5 \mathrm{~Hz}, 2 \mathrm{H}), 6.85(\mathrm{~d}, J=8.1 \mathrm{~Hz}, 2 \mathrm{H}), 5.85(\mathrm{dr}, 1 \mathrm{H})$.

${ }^{13}$ C NMR (101 MHz, CDCl $) \delta 163.0,161.7,152.7,141.3,140.5,137.8,135.1,133.4,132.1,130.6$, $129.7,129.6,128.7,128.7,128.33,128.29,128.0,126.7,125.5,125.0,124.7,123.9,123.7,123.1,122.6$, 116.9, 116.3, 116.2, 107.6.

HRMS (ESI) calculated for $[\mathrm{M}+\mathrm{H}]^{+} \mathrm{C}_{33} \mathrm{H}_{24} \mathrm{NO}_{3}{ }^{+}, \mathrm{m} / \mathrm{z}: 482.1756$, found: 482.1750 .

M.P. $126-128^{\circ} \mathrm{C}$.

$[\alpha]^{20}{ }_{\mathrm{D}}=103^{\circ}\left(\mathrm{c}=0.2, \mathrm{CHCl}_{3}\right)$.

HPLC analysis: HPLC DAICEL CHIRALPAK AD-H, hexane/isopropyl alcohol = 80/20, flow rate = 1 
$\mathrm{mL} / \mathrm{min}, \lambda=220 \mathrm{~nm}), \mathrm{tR}($ major $)=16.0 \mathrm{~min}, \mathrm{tR}($ minor $)=12.1 \mathrm{~min}$, ee $=94 \%$.

Chiral HPLC spectrum of racemic 6aa

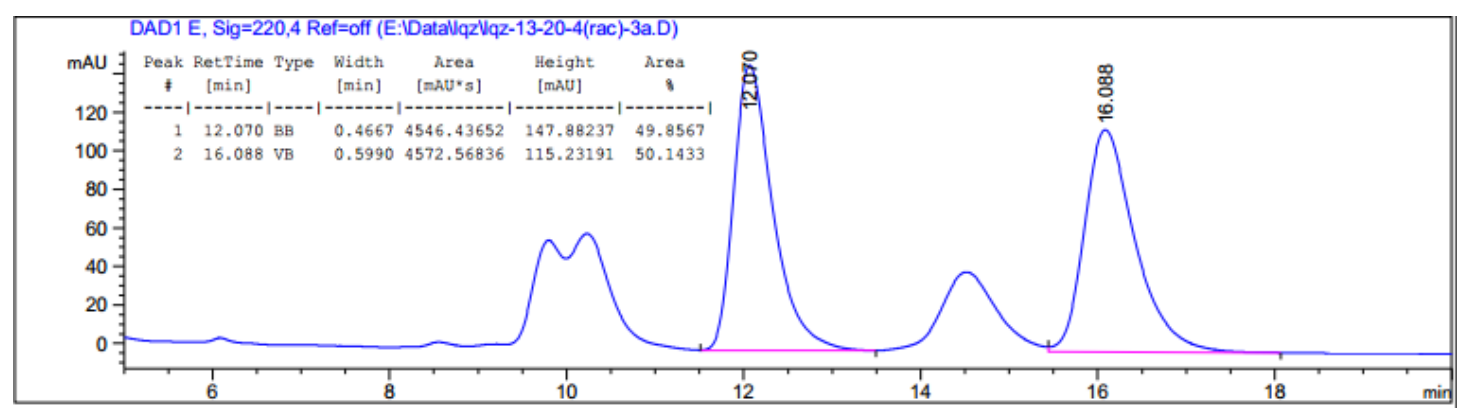

Chiral HPLC spectrum of $\mathbf{6 a a}$
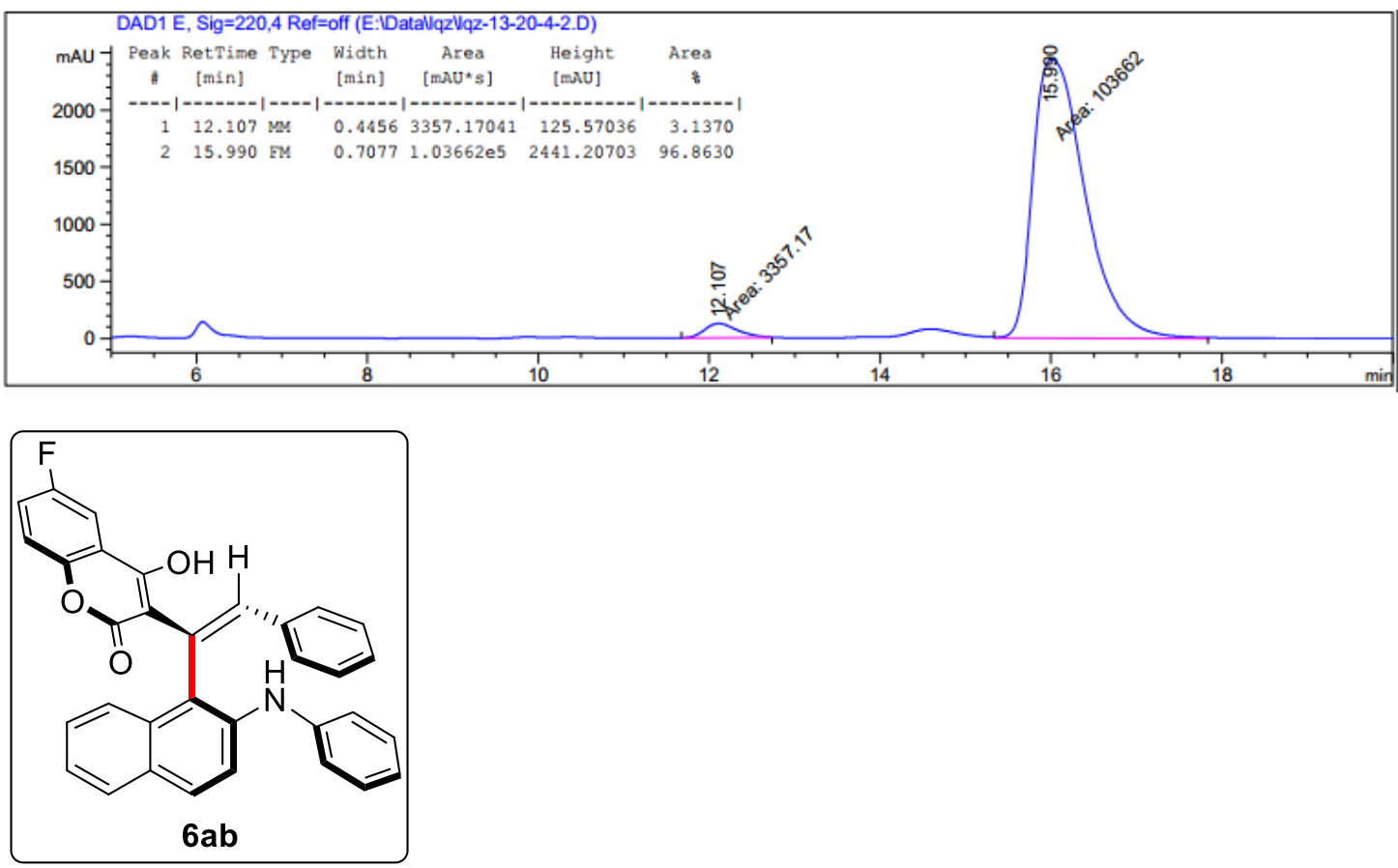

(aS)-(E)-6-fluoro-4-hydroxy-3-(2-phenyl-1-(2-(phenylamino)naphthalen-1-yl)vinyl)-2Hchromen-2-one (6ab)

According to procedure D, 6ab was obtained as a yellow solid, $28.0 \mathrm{mg}$ ( $56 \%$ yield, $97 \%$ ee).

${ }^{1}$ H NMR (400 MHz, CDCl $) \delta 10.63(\mathrm{br}, 1 \mathrm{H}), 8.41(\mathrm{~d}, J=8.5 \mathrm{~Hz}, 1 \mathrm{H}), 7.79$ (d, $\left.J=8.1 \mathrm{~Hz}, 1 \mathrm{H}\right), 7.73$ $(\mathrm{d}, J=9.0 \mathrm{~Hz}, 1 \mathrm{H}), 7.54(\mathrm{dd}, J=8.5,7.0 \mathrm{~Hz}, 1 \mathrm{H}), 7.46-7.39(\mathrm{~m}, 3 \mathrm{H}), 7.35(\mathrm{t}, J=7.7 \mathrm{~Hz}, 2 \mathrm{H}), 7.27-$ $7.23(\mathrm{~m}, 1 \mathrm{H}), 7.21-7.18(\mathrm{~m}, 3 \mathrm{H}), 7.16-7.09(\mathrm{~m}, 3 \mathrm{H}), 7.01(\mathrm{~d}, J=7.8 \mathrm{~Hz}, 2 \mathrm{H}), 6.85(\mathrm{~d}, J=7.8 \mathrm{~Hz}$, 2H), 5.85 (br, 1H).

${ }^{13}$ C NMR (101 MHz, CDCl 3$) \delta 162.7,160.9,148.8,141.5,140.3,137.8,134.9,133.3,130.7,129.8$, $129.7,128.8,128.7,128.343128 .1,126.4,125.5,125.1,124.9,123.0,122.8,119.7,119.4,117.9,117.788$, 117.2, 116.9, 109.7, 109.4, 108.1.

HRMS (ESI) calculated for $[\mathrm{M}+\mathrm{H}]^{+} \mathrm{C}_{33} \mathrm{H}_{23} \mathrm{FNO}_{3}{ }^{+}, \mathrm{m} / \mathrm{z}$ : 500.1662, found: 500.1668.

M.P. $121-123^{\circ} \mathrm{C}$.

$[\alpha]^{20}{ }_{\mathrm{D}}=3455^{\circ}\left(\mathrm{c}=0.2, \mathrm{CHCl}_{3}\right)$.

HPLC analysis: HPLC DAICEL CHIRALPAK AD-H, hexane/isopropyl alcohol = 80/20, flow rate = 1 $\mathrm{mL} / \mathrm{min}, \lambda=254 \mathrm{~nm})$, $\mathrm{tR}($ major $)=14.9 \mathrm{~min}, \mathrm{tR}($ minor $)=11.3 \mathrm{~min}$, ee $=97 \%$.

Chiral HPLC spectrum of racemic $\mathbf{6} \boldsymbol{a b}$ 


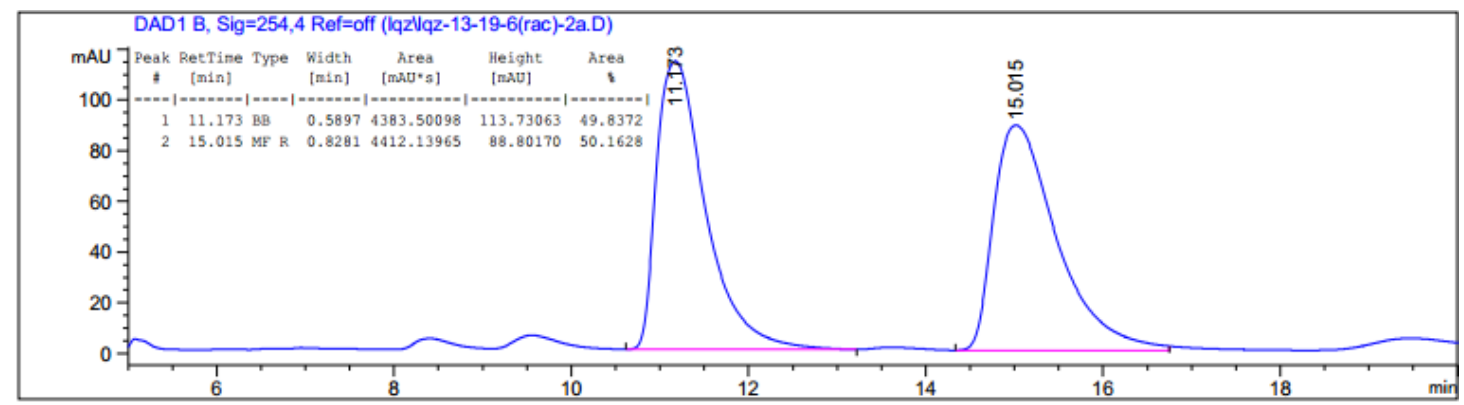

Chiral HPLC spectrum of $\mathbf{6 a b}$
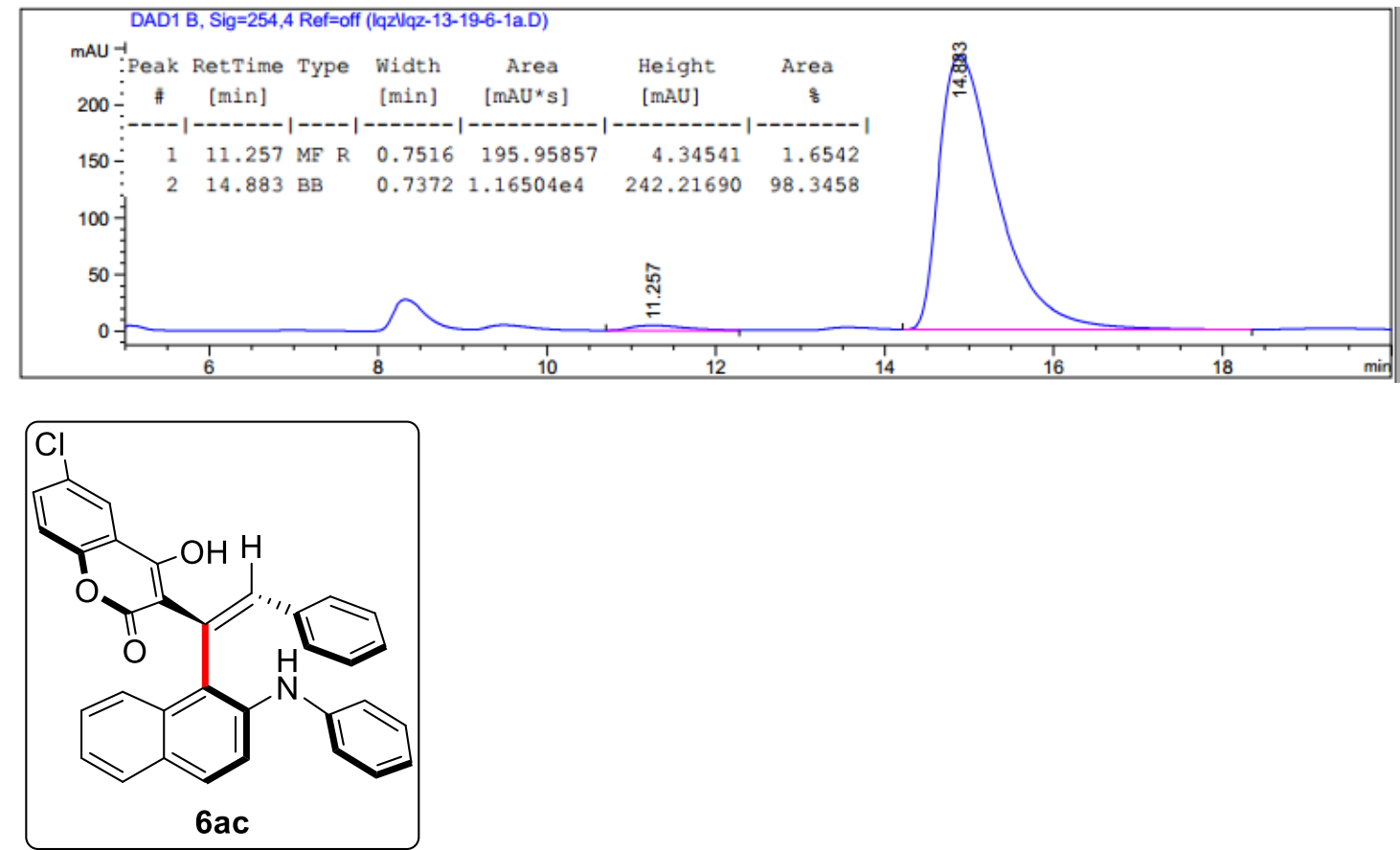

(aS)-(E)-6-chloro-4-hydroxy-3-(2-phenyl-1-(2-(phenylamino)naphthalen-1-yl)vinyl)-2Hchromen-2-one (6ac)

According to procedure $\mathbf{D}, \mathbf{6 a c}$ was obtained as a yellow solid, $24.7 \mathrm{mg}$ (48\% yield, $92 \% \mathrm{ee}$ ).

${ }^{1} \mathbf{H}$ NMR (400 MHz, CDCl $) \delta 10.93(\mathrm{br}, 1 \mathrm{H}), 8.39(\mathrm{~d}, J=8.5 \mathrm{~Hz}, 1 \mathrm{H}), 7.79(\mathrm{~d}, J=8.0 \mathrm{~Hz}, 1 \mathrm{H}), 7.76-$ $7.72(\mathrm{~m}, 2 \mathrm{H}), 7.54(\mathrm{t}, J=7.2 \mathrm{~Hz}, 1 \mathrm{H}), 7.43-7.39(\mathrm{~m}, 3 \mathrm{H}), 7.36(\mathrm{t}, J=7.8 \mathrm{~Hz}, 2 \mathrm{H}), 7.26(\mathrm{~s}, 1 \mathrm{H}), 7.23-$ 7.19 (m, 3H), $7.15-7.09$ (m, 3H), $7.01(\mathrm{~d}, J=7.2 \mathrm{~Hz}, 2 \mathrm{H}), 6.86(\mathrm{~d}, J=7.4 \mathrm{~Hz}, 2 \mathrm{H}), 5.79(\mathrm{br}, 1 \mathrm{H})$.

${ }^{13} \mathbf{C}$ NMR (101 MHz, $\left.\mathbf{C D C l}_{3}\right) \delta 162.5,160.7,151.1,141.5,140.3,137.8,134.9,133.3,132.0,130.7$, $129.8,129.7,129.2,128.8,128.7,128.3,128.1,126.3,125.4,125.1,125.0,123.5,123.1,122.8,117.7$, 117.4, 116.9, 108.2.

HRMS (ESI) calculated for $[\mathrm{M}+\mathrm{H}]^{+} \mathrm{C}_{33} \mathrm{H}_{23} \mathrm{ClNO}_{3}{ }^{+}$, m/z: 516.1366 , found: 516.1364 .

M.P. $127-130^{\circ} \mathrm{C}$.

$[\alpha]^{20}{ }_{\mathrm{D}}=246^{\circ}(\mathrm{c}=0.2, \mathrm{CHCl} 3)$.

HPLC analysis: HPLC DAICEL CHIRALPAK AD-H, hexane/isopropyl alcohol $=90 / 10$, flow rate $=$ $0.8 \mathrm{~mL} / \mathrm{min}, \lambda=254 \mathrm{~nm}$ ), $\mathrm{tR}$ (major) $=43.1 \mathrm{~min}, \mathrm{tR}(\operatorname{minor})=40.1 \mathrm{~min}$, ee $=92 \%$.

Chiral HPLC spectrum of racemic 6 ac 


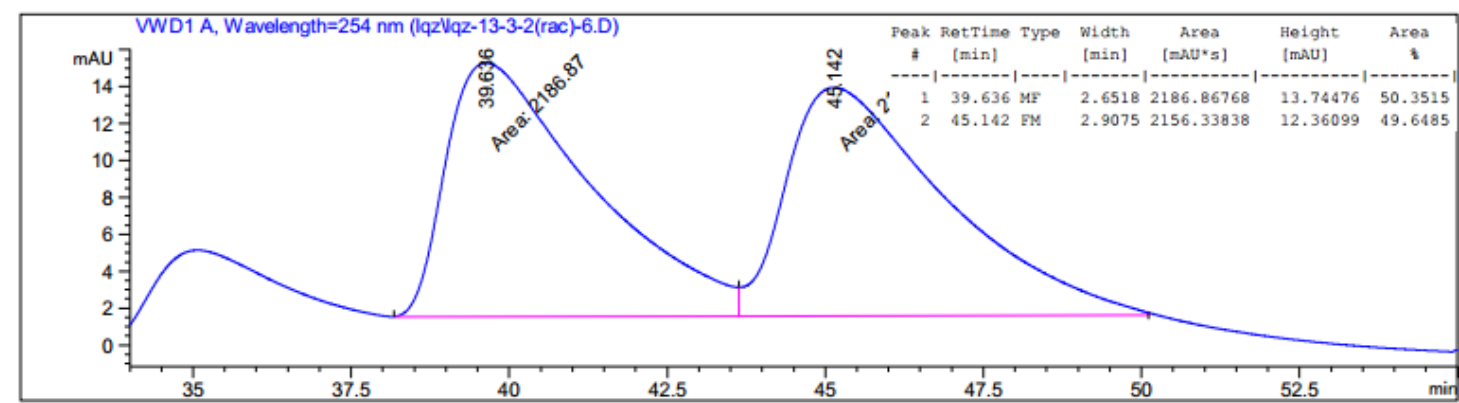

Chiral HPLC spectrum of $6 a c$
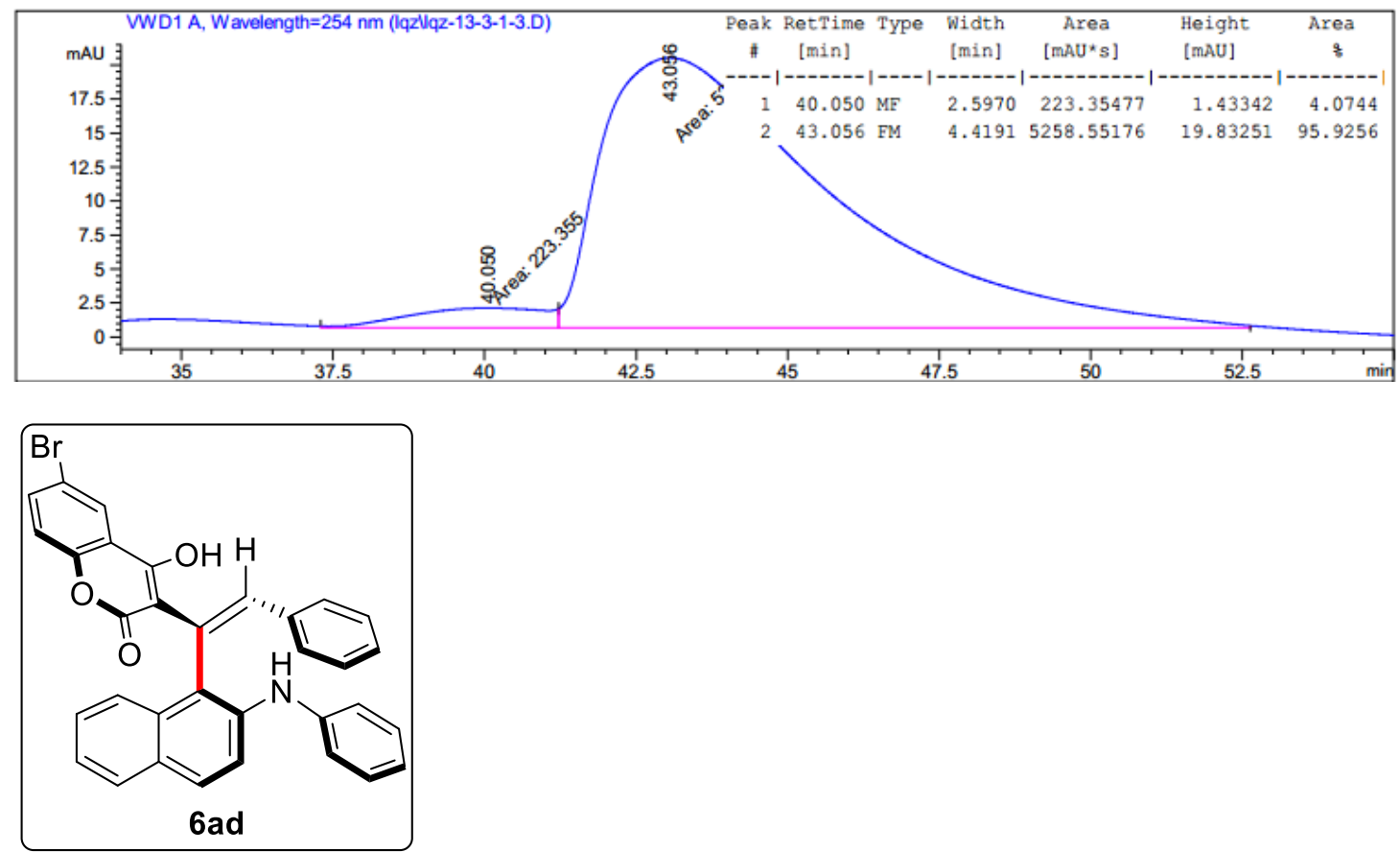

(aS)-(E)-6-bromo-4-hydroxy-3-(2-phenyl-1-(2-(phenylamino)naphthalen-1-yl)vinyl)-2H-

chromen-2-one (6ad)

According to procedure $\mathbf{D}, \mathbf{6 a d}$ was obtained as a yellow solid, $35.2 \mathrm{mg}$ ( $63 \%$ yield, $97 \%$ ee).

${ }^{1} \mathbf{H}$ NMR (400 MHz, CDCl $) \delta 10.93(\mathrm{br}, 1 \mathrm{H}), 7.90(\mathrm{~d}, J=2.3 \mathrm{~Hz}, 1 \mathrm{H}), 7.79$ (d, $\left.J=8.1 \mathrm{~Hz}, 1 \mathrm{H}\right), 7.73$ $(\mathrm{d}, J=8.9 \mathrm{~Hz}, 1 \mathrm{H}), 7.55-7.51(\mathrm{~m}, 2 \mathrm{H}), 7.41(\mathrm{~d}, J=10.1 \mathrm{~Hz}, 2 \mathrm{H}), 7.35(\mathrm{t}, J=7.7 \mathrm{~Hz}, 2 \mathrm{H}), 7.22-7.09$ (m, 6H), 7.01 (d, $J=7.8 \mathrm{~Hz}, 2 \mathrm{H}), 6.86(\mathrm{~d}, J=7.8 \mathrm{~Hz}, 2 \mathrm{H}), 5.81(\mathrm{br}, 1 \mathrm{H})$.

${ }^{13}$ C NMR (101 MHz, CDCl $) \delta 162.4,160.6,151.5,141.6,140.3,137.8,134.9,134.8,133.3,130.7$, $129.82,129.75,128.8,128.7,128.343$ 128.1, 126.5, 126.3, 125.4, 125.1, 125.0, 123.1, 122.8, 118.0, $117.9,116.9,116.5,108.2$.

HRMS (ESI) calculated for $[\mathrm{M}+\mathrm{H}]^{+} \mathrm{C}_{33} \mathrm{H}_{23} \mathrm{BrNO}_{3}{ }^{+}, \mathrm{m} / \mathrm{z}$ : 560.0861, found: 560.0863 .

M.P. $127-129^{\circ} \mathrm{C}$.

$[\alpha]^{20}{ }_{\mathrm{D}}=346^{\circ}\left(\mathrm{c}=0.2, \mathrm{CHCl}_{3}\right)$.

HPLC analysis: HPLC DAICEL CHIRALPAK AD-H, hexane/isopropyl alcohol $=60 / 40$, flow rate $=1$ $\mathrm{mL} / \mathrm{min}, \lambda=365 \mathrm{~nm}$ ), $\mathrm{tR}$ (major) $=7.5 \mathrm{~min}$, $\mathrm{tR}($ minor $)=6.5 \mathrm{~min}$, ee $=97 \%$.

Chiral HPLC spectrum of racemic $\boldsymbol{6} \boldsymbol{a d}$ 


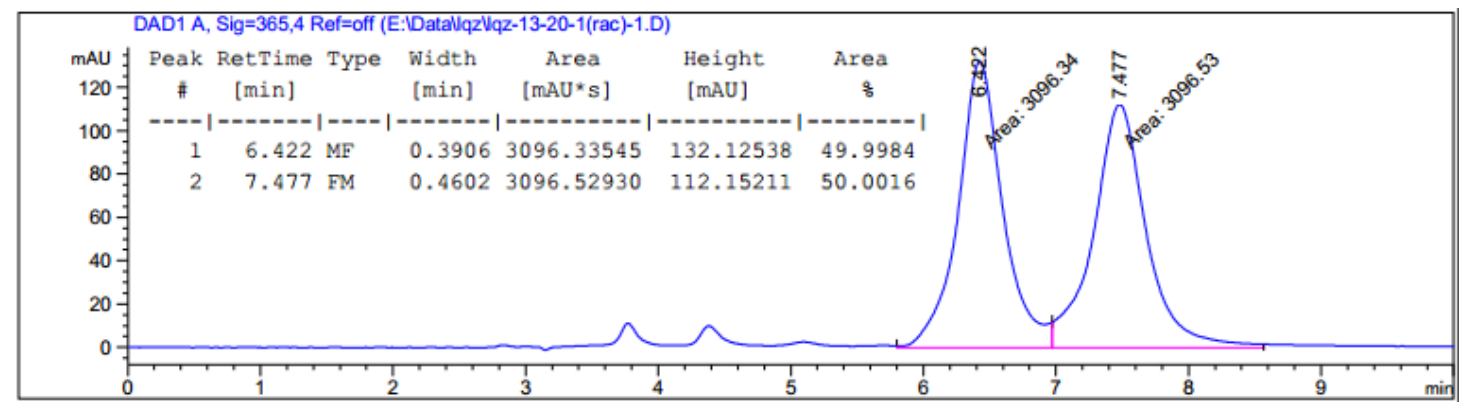

Chiral HPLC spectrum of $\mathbf{6 a d}$
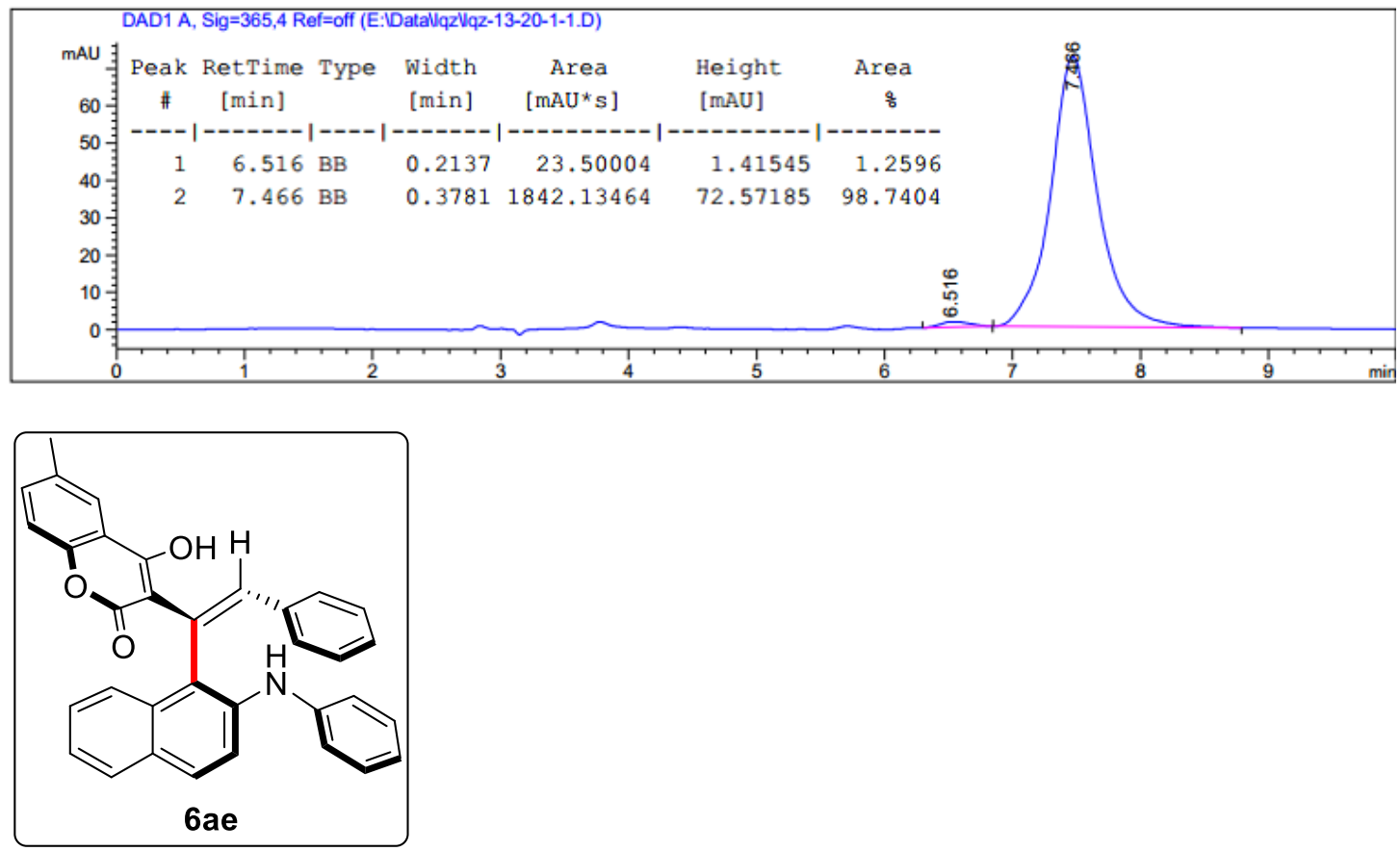

(aS)-(E)-4-hydroxy-6-methyl-3-(2-phenyl-1-(2-(phenylamino)naphthalen-1-yl)vinyl)-2Hchromen-2-one (6ae)

According to procedure D, 6ae was obtained as a yellow solid, $29.4 \mathrm{mg}$ (59\% yield, 92\% ee).

${ }^{1}$ H NMR (400 MHz, CDCl $) \delta 10.44(\mathrm{br}, 1 \mathrm{H}), 8.40(\mathrm{~d}, J=8.5 \mathrm{~Hz}, 1 \mathrm{H}), 7.78(\mathrm{~d}, J=8.1 \mathrm{~Hz}, 1 \mathrm{H}), 7.72$ $(\mathrm{d}, J=9.0 \mathrm{~Hz}, 1 \mathrm{H}), 7.56(\mathrm{~s}, 2 \mathrm{H}), 7.52(\mathrm{t}, J=7.7 \mathrm{~Hz}, 1 \mathrm{H}), 7.40(\mathrm{~d}, J=5.4 \mathrm{~Hz}, 2 \mathrm{H}), 7.37-7.32(\mathrm{~m}, 2 \mathrm{H})$, $7.29-7.27(\mathrm{~m}, 1 \mathrm{H}), 7.19-7.10(\mathrm{~m}, 6 \mathrm{H}), 7.01(\mathrm{~d}, J=7.2 \mathrm{~Hz}, 2 \mathrm{H}), 6.85(\mathrm{~d}, J=8.0 \mathrm{~Hz}, 2 \mathrm{H}), 5.85$ (br, $1 \mathrm{H}), 2.33$ (s, 3H).

${ }^{13}$ C NMR (101 MHz, CDCl $) \delta 163.2,161.6,150.9,141.3,140.6,137.8,135.1,133.40,133.36,133.1$, $130.6,129.7,129.6,128.6,128.33,128.27,128.0,126.8,125.5,124.9,124.6,123.5,123.1,122.5,116.9$, $116.0,115.8,107.5,20.9$.

HRMS (ESI) calculated for $[\mathrm{M}+\mathrm{H}]^{+} \mathrm{C}_{34} \mathrm{H}_{26} \mathrm{NO}_{3}{ }^{+}, \mathrm{m} / \mathrm{z}: 496.1913$, found: 496.1905 .

M.P. $124-126^{\circ} \mathrm{C}$.

$[\alpha]^{20}{ }_{\mathrm{D}}=348^{\circ}\left(\mathrm{c}=0.2, \mathrm{CHCl}_{3}\right)$.

HPLC analysis: HPLC DAICEL CHIRALPAK AD-H, hexane/isopropyl alcohol $=80 / 20$, flow rate $=1$ $\mathrm{mL} / \mathrm{min}, \lambda=365 \mathrm{~nm}$ ), tR (major) $=21.5 \mathrm{~min}, \mathrm{tR}($ minor $)=15.8 \mathrm{~min}$, ee $=92 \%$.

Chiral HPLC spectrum of racemic 6ae 


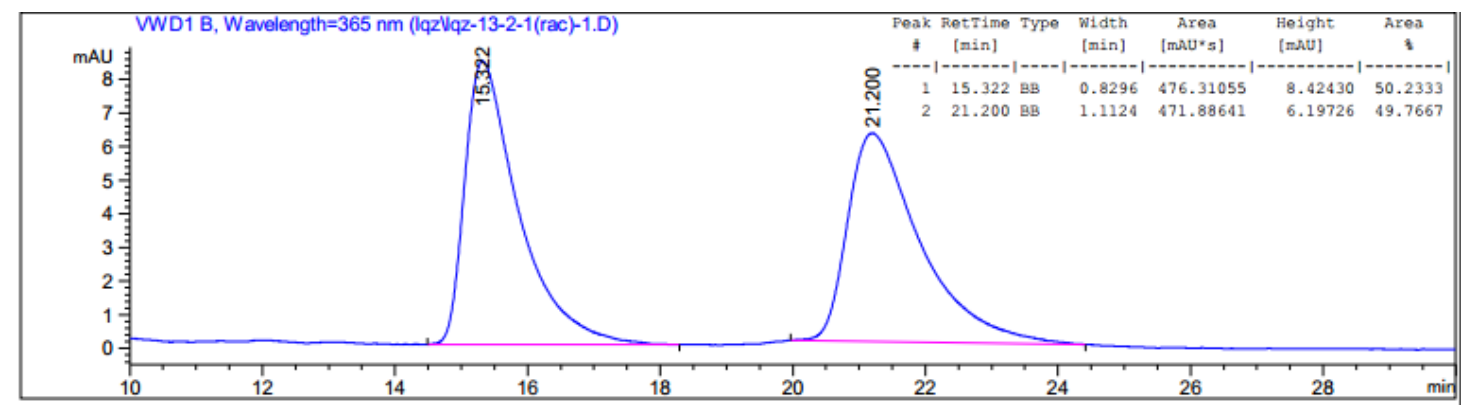

Chiral HPLC spectrum of 6 ae
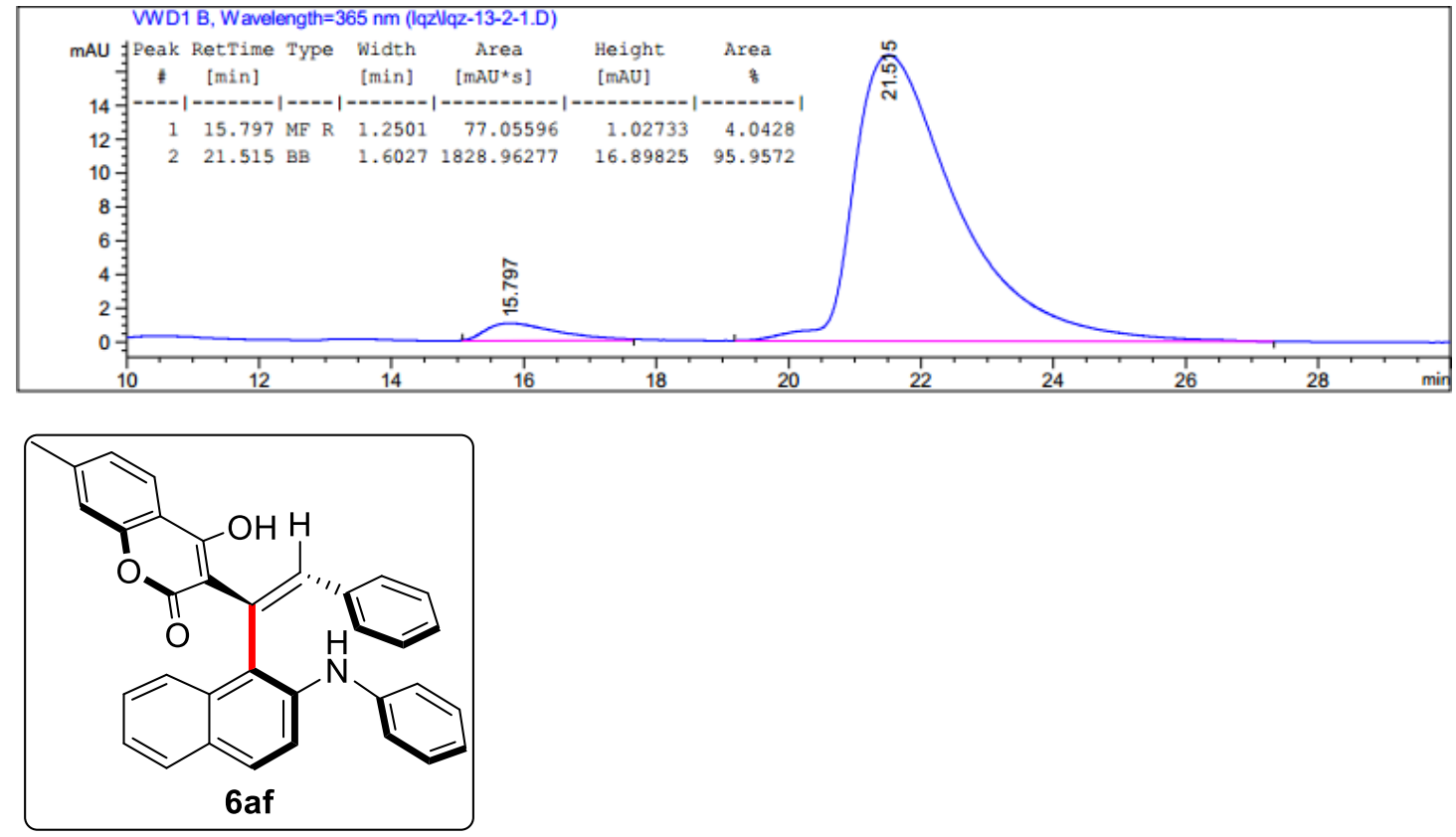

(aS)-(E)-4-hydroxy-7-methyl-3-(2-phenyl-1-(2-(phenylamino)naphthalen-1-yl)vinyl)-2Hchromen-2-one (6af)

According to procedure D, 6af was obtained as a yellow solid, $41.6 \mathrm{mg}$ ( $84 \%$ yield, $95 \%$ ee).

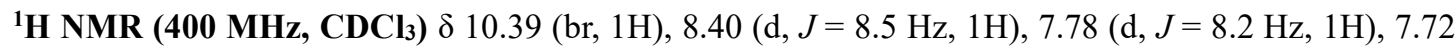
$(\mathrm{d}, J=9.0 \mathrm{~Hz}, 1 \mathrm{H}), 7.64(\mathrm{~d}, J=8.1 \mathrm{~Hz}, 1 \mathrm{H}), 7.52(\mathrm{t}, J=7.7 \mathrm{~Hz}, 1 \mathrm{H}), 7.40(\mathrm{~d}, J=2.7 \mathrm{~Hz}, 2 \mathrm{H}), 7.33(\mathrm{t}, J$ $=7.7 \mathrm{~Hz}, 2 \mathrm{H}), 7.19-7.09(\mathrm{~m}, 6 \mathrm{H}), 7.01-6.98(\mathrm{~m}, 3 \mathrm{H}), 6.85(\mathrm{~d}, J=7.8 \mathrm{~Hz}, 2 \mathrm{H}), 5.86(\mathrm{br}, 1 \mathrm{H}), 2.40(\mathrm{~s}$, $3 \mathrm{H})$.

${ }^{13}$ C NMR (101 MHz, CDCl $) \delta 163.2,161.9,152.9,143.3,141.2,140.6,137.8,135.2,133.4,130.6$, $129.7,129.6,128.6,128.34,128.32,128.27,128.0,126.8,125.5,125.0,124.9,124.6,123.6,123.1,122.5$, $116.9,116.4,113.6,106.7,21.7$.

HRMS (ESI) calculated for $[\mathrm{M}+\mathrm{H}]^{+} \mathrm{C}_{34} \mathrm{H}_{26} \mathrm{NO}_{3}{ }^{+}, \mathrm{m} / \mathrm{z}$ : 496.1913, found: 496.1917.

M.P. $120-122^{\circ} \mathrm{C}$.

$[\alpha]^{20}{ }_{\mathrm{D}}=182^{\circ}\left(\mathrm{c}=0.2, \mathrm{CHCl}_{3}\right)$.

HPLC analysis: HPLC DAICEL CHIRALPAK AD-H, hexane/isopropyl alcohol = 95/5, flow rate $=1$ $\mathrm{mL} / \mathrm{min}, \lambda=220 \mathrm{~nm})$, tR (major) $=21.5 \mathrm{~min}, \mathrm{tR}($ minor $)=6.4 \mathrm{~min}$, ee $=95 \%$.

Chiral HPLC spectrum of racemic 6 af 


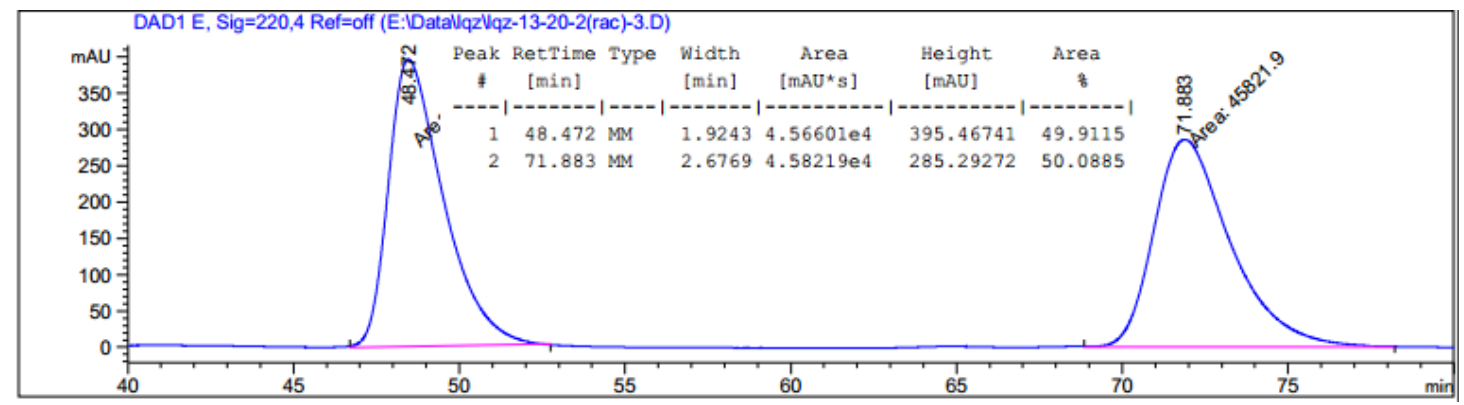

Chiral HPLC spectrum of $\mathbf{6}$ af
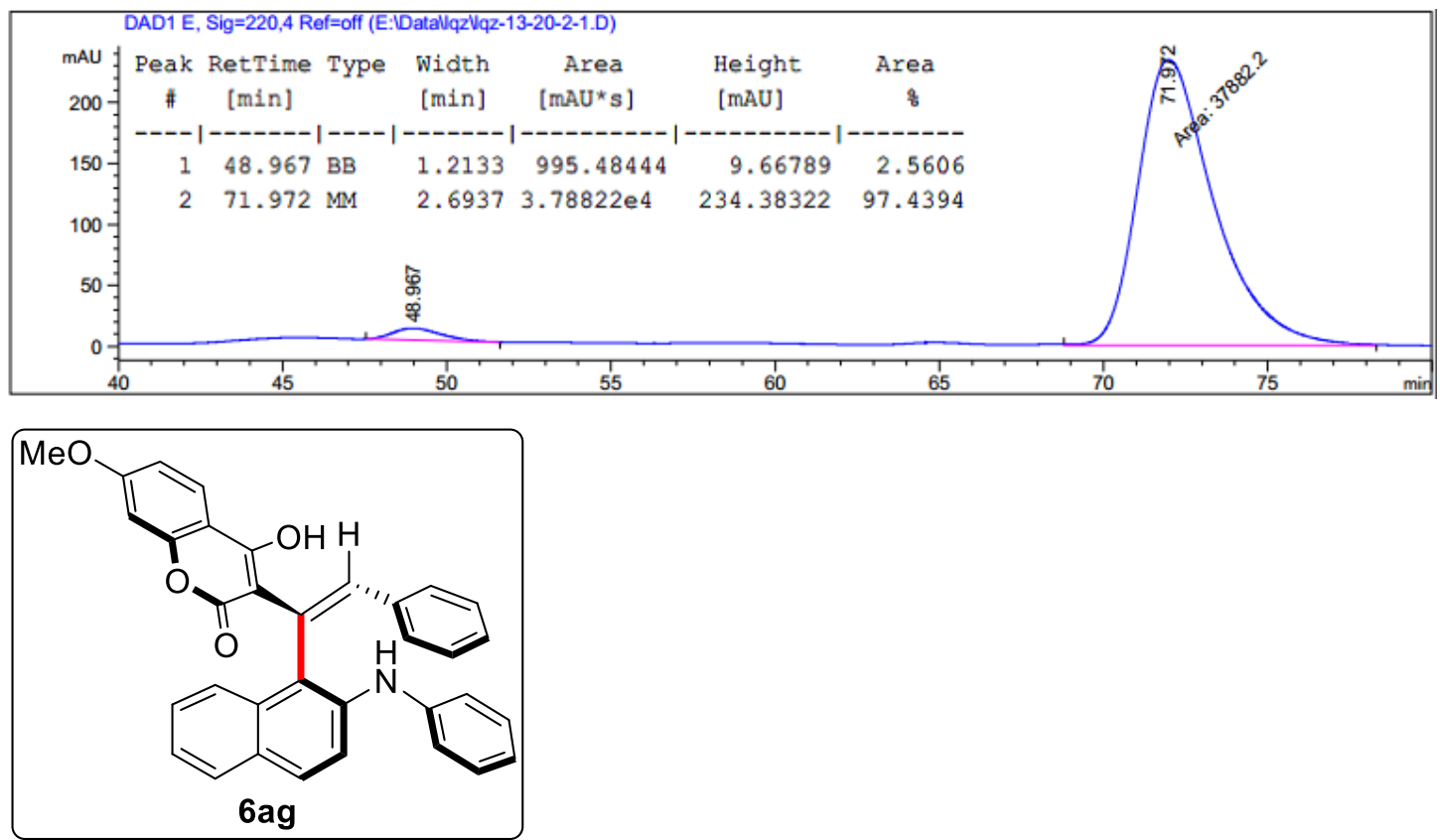

(aS)-(E)-4-hydroxy-7-methoxy-3-(2-phenyl-1-(2-(phenylamino)naphthalen-1-yl)vinyl)-2Hchromen-2-one (6ag)

According to procedure $\mathbf{D}, \mathbf{6 a g}$ was obtained as a yellow solid, $23.0 \mathrm{mg}$ (45\% yield, $95 \%$ ee).

${ }^{1}$ H NMR (400 MHz, CDCl $) \delta 10.34(\mathrm{~s}, 1 \mathrm{H}), 8.40(\mathrm{~d}, J=8.5 \mathrm{~Hz}, 1 \mathrm{H}), 7.78(\mathrm{~d}, J=8.1 \mathrm{~Hz}, 1 \mathrm{H}), 7.72(\mathrm{~d}$, $J=8.9 \mathrm{~Hz}, 1 \mathrm{H}), 7.66(\mathrm{~d}, J=8.7 \mathrm{~Hz}, 1 \mathrm{H}), 7.53(\mathrm{t}, J=7.7 \mathrm{~Hz}, 1 \mathrm{H}), 7.40(\mathrm{~d}, J=7.7 \mathrm{~Hz}, 2 \mathrm{H}), 7.33(\mathrm{t}, J=$ $7.8 \mathrm{~Hz}, 2 \mathrm{H}), 7.23-7.10(\mathrm{~m}, 5 \mathrm{H}), 7.00(\mathrm{~d}, J=7.6 \mathrm{~Hz}, 2 \mathrm{H}), 6.84(\mathrm{~d}, J=7.8 \mathrm{~Hz}, 2 \mathrm{H}), 6.75(\mathrm{~d}, J=7.8 \mathrm{~Hz}$, $2 \mathrm{H}), 5.91(\mathrm{br}, 1 \mathrm{H}), 3.83(\mathrm{~s}, 3 \mathrm{H})$.

${ }^{13} \mathbf{C}$ NMR (101 MHz, CDCl $) \delta 163.4,163.1,162.1,154.5,141.1,140.6,137.8,135.2,133.4,130.6$, $129.7,129.5,128.61,128.55,128.30,128.27,127.9,126.9,125.5,125.0,124.9,124.6,123.3,122.5$, $116.9,112.1,109.4,105.2,100.0,55.7$.

HRMS (ESI) calculated for $[\mathrm{M}+\mathrm{H}]^{+} \mathrm{C}_{34} \mathrm{H}_{26} \mathrm{NO}_{4}{ }^{+}, \mathrm{m} / \mathrm{z}$ : 512.1862, found: 512.1866.

M.P. $109-113^{\circ} \mathrm{C}$.

$[\alpha]^{20}{ }_{\mathrm{D}}=186^{\circ}\left(\mathrm{c}=0.2, \mathrm{CHCl}_{3}\right)$.

HPLC analysis: HPLC DAICEL CHIRALPAK AD-H, hexane/isopropyl alcohol $=80 / 20$, flow rate $=1$ $\mathrm{mL} / \mathrm{min}, \lambda=254 \mathrm{~nm}), \mathrm{tr}($ major $)=21.5 \mathrm{~min}, \mathrm{tR}($ minor $)=15.6 \mathrm{~min}, \mathrm{ee}=95 \%$.

Chiral HPLC spectrum of racemic $\mathbf{6 a g}$ 


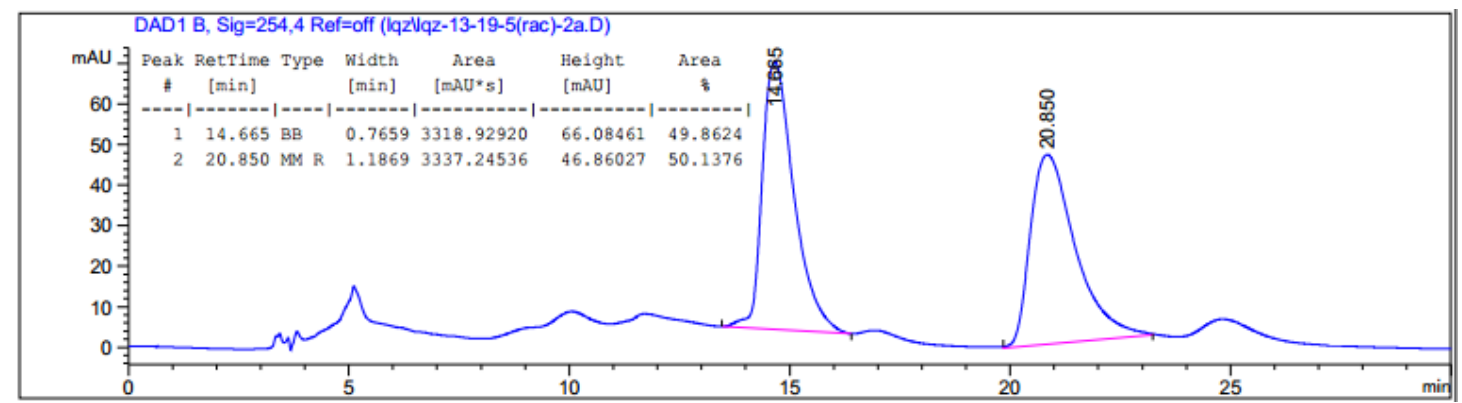

Chiral HPLC spectrum of 6 ag
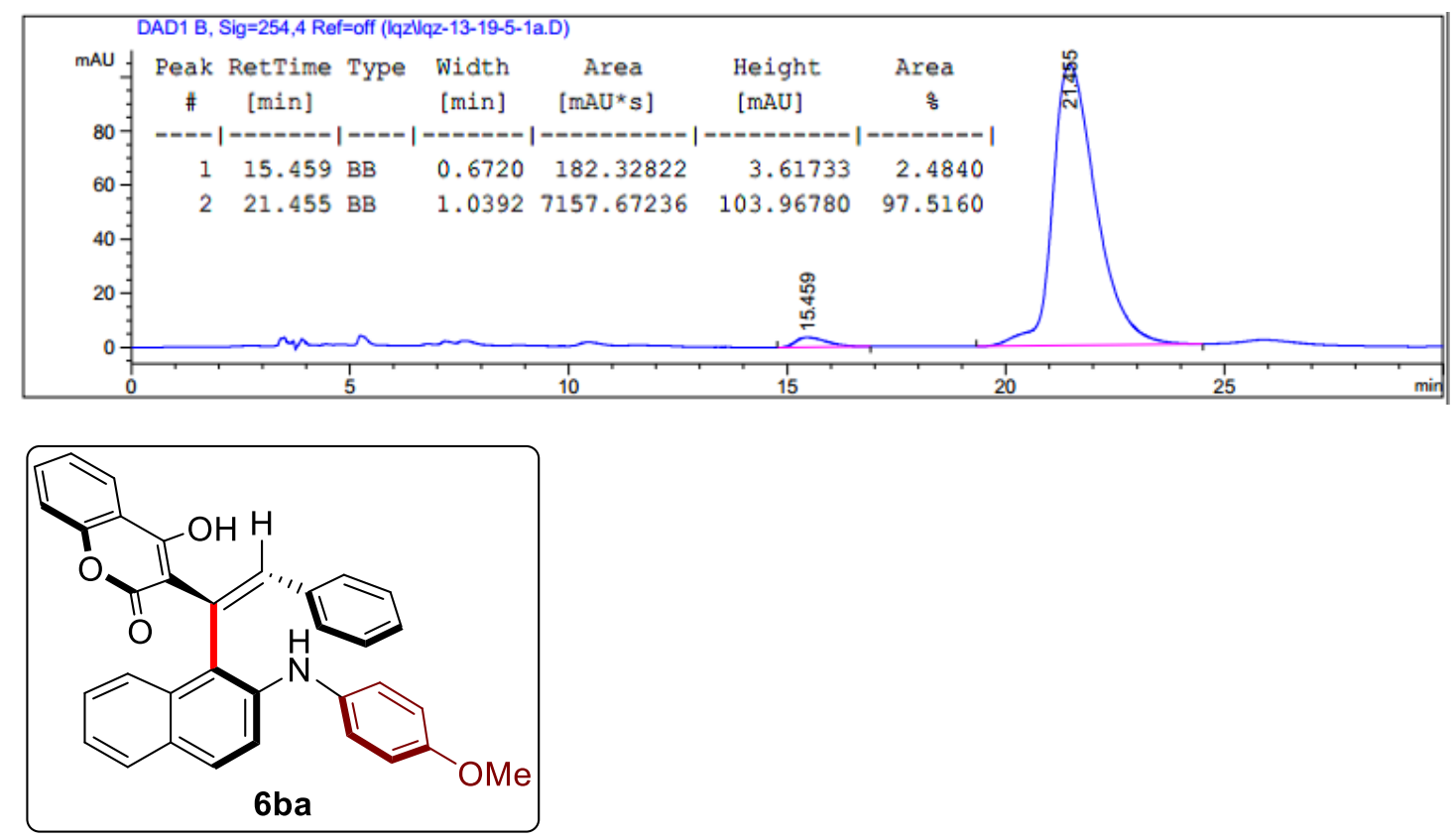

(aS)-(E)-4-hydroxy-3-(1-(2-((4-methoxyphenyl)amino)naphthalen-1-yl)-2-phenylvinyl)-2Hchromen-2-one (6ba)

According to procedure D, 6ba was obtained as a yellow solid, $40.0 \mathrm{mg}$ (76\% yield, $93 \%$ ee).

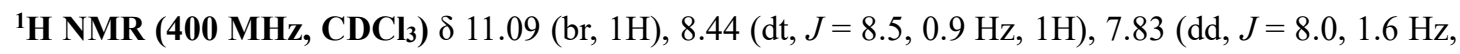
$1 \mathrm{H}), 7.80-7.78(\mathrm{~m}, 1 \mathrm{H}), 7.72(\mathrm{~d}, J=8.8 \mathrm{~Hz}, 1 \mathrm{H}), 7.55(\mathrm{ddd}, J=8.4,6.8,1.4 \mathrm{~Hz}, 1 \mathrm{H}), 7.50(\mathrm{ddd}, J=$ 8.8, 7.3, 1.6 Hz, 1H), $7.43-7.39(\mathrm{~m}, 2 \mathrm{H}), 7.31(\mathrm{dd}, J=8.4,1.1 \mathrm{~Hz}, 1 \mathrm{H}), 7.25-7.19(\mathrm{~m}, 2 \mathrm{H}), 7.17-$ $7.13(\mathrm{~m}, 2 \mathrm{H}), 7.06-7.03(\mathrm{~m}, 2 \mathrm{H}), 6.96(\mathrm{~d}, J=8.9 \mathrm{~Hz}, 1 \mathrm{H}), 6.92-6.89(\mathrm{~m}, 2 \mathrm{H}), 6.81-6.79(\mathrm{~m}, 2 \mathrm{H})$, 5.78 (br, $1 \mathrm{H}), 3.84(\mathrm{~s}, 3 \mathrm{H})$.

${ }^{13}$ C NMR (101 MHz, CDCl 3$) \delta 163.1,161.8,157.5,152.7,141.1,138.8,135.1,133.3,133.0,132.0$, $130.4,129.6,128.6,128.4,128.2$, 128.0, 126.8, 125.50, 125.47, 124.8, 124.0, 123.7, 122.2, 116.4, 116.3, $116.2,115.0,114.5,107.5,55.6$.

HRMS (ESI) calculated for $[\mathrm{M}+\mathrm{H}]^{+} \mathrm{C}_{34} \mathrm{H}_{26} \mathrm{NO}_{4}{ }^{+}, \mathrm{m} / \mathrm{z}: 512.1862$, found: 512.1865.

M.P. $126-128^{\circ} \mathrm{C}$.

$[\alpha]^{20}{ }_{\mathrm{D}}=114^{\circ}\left(\mathrm{c}=0.2, \mathrm{CHCl}_{3}\right)$.

HPLC analysis: HPLC DAICEL CHIRALPAK AD-H, hexane/isopropyl alcohol $=60 / 40$, flow rate $=1$ $\mathrm{mL} / \mathrm{min}, \lambda=254 \mathrm{~nm})$, tR (major) $=11.2 \mathrm{~min}, \mathrm{tR}($ minor $)=9.4 \mathrm{~min}$, ee $=93 \%$.

Chiral HPLC spectrum of racemic $6 \mathbf{b a}$ 


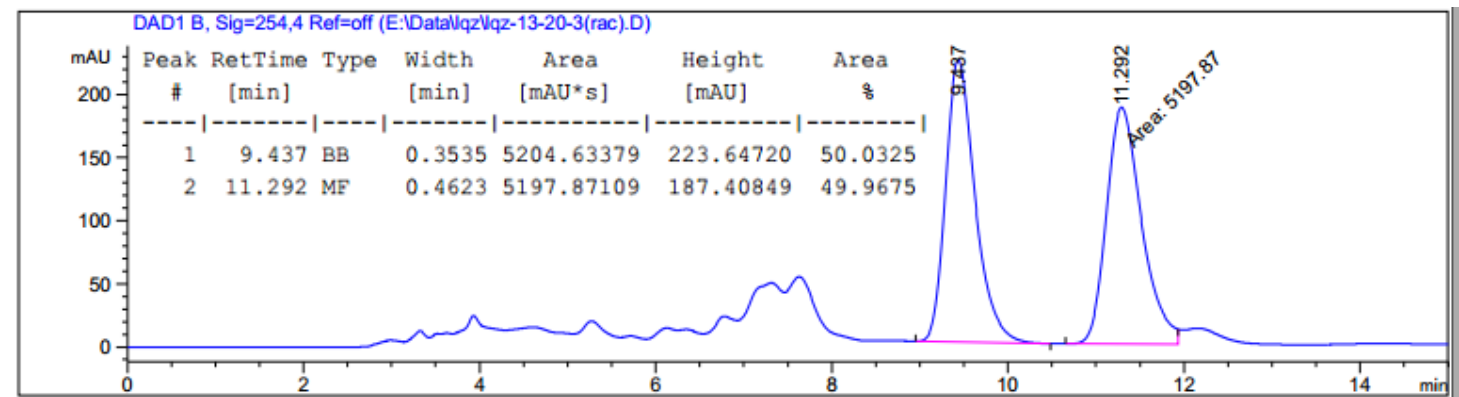

Chiral HPLC spectrum of $\mathbf{6 b a}$
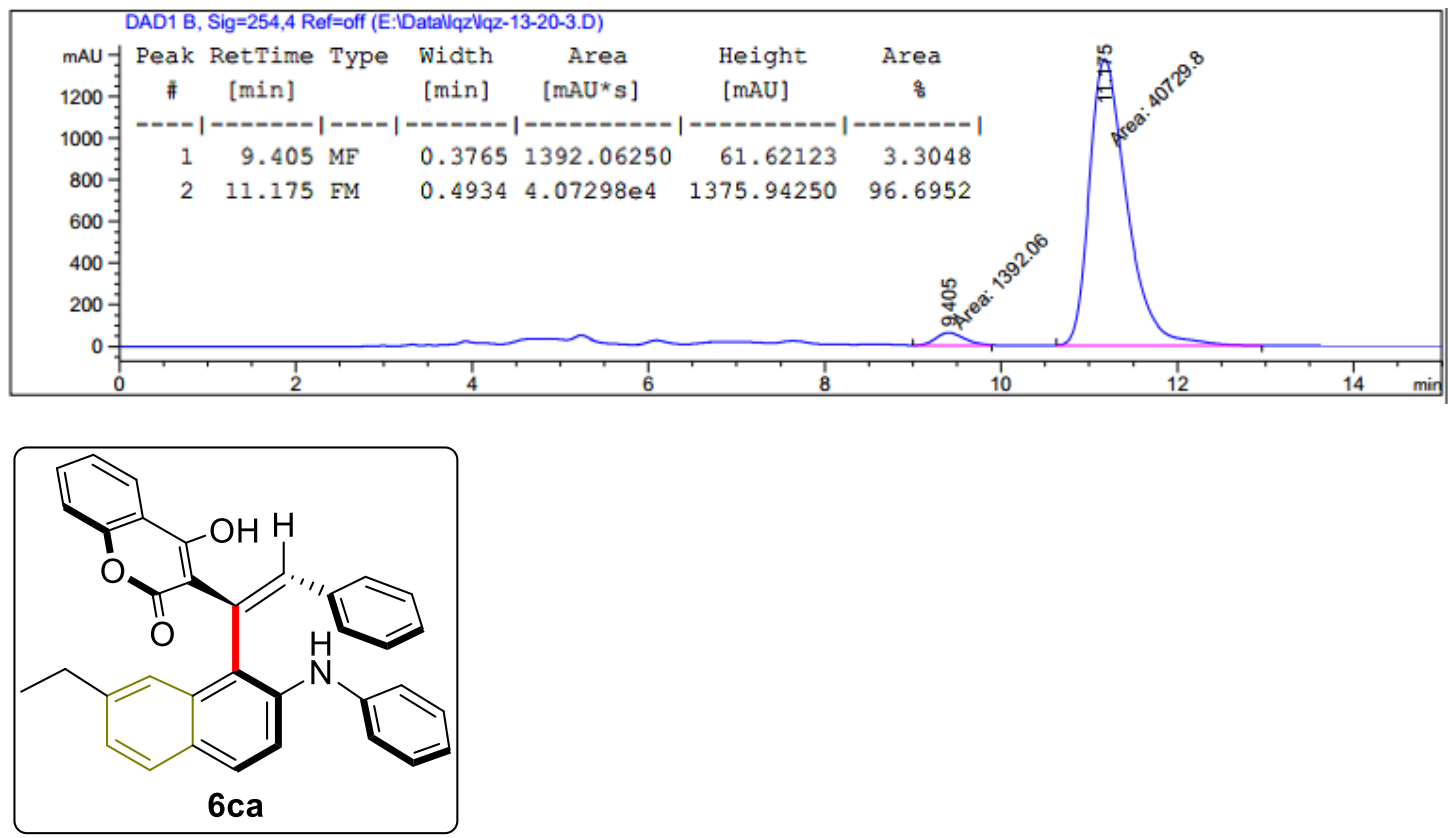

(aS)-(E)-3-(1-(7-ethyl-2-(phenylamino)naphthalen-1-yl)-2-phenylvinyl)-4-hydroxy-2Hchromen-2-one (6ca)

According to procedure D, 6ca was obtained as a yellow solid, $42.0 \mathrm{mg}$ ( $83 \%$ yield, $96 \%$ ee).

${ }^{1}$ H NMR (400 MHz, CDCl $) \delta 10.57$ (br, 1H), 8.25 (s, 1H), 7.77 (d, $\left.J=7.9 \mathrm{~Hz}, 1 \mathrm{H}\right), 7.69$ (dd, $J=10.8$, $8.6 \mathrm{~Hz}, 2 \mathrm{H}), 7.49-7.43(\mathrm{~m}, 1 \mathrm{H}), 7.39(\mathrm{~s}, 1 \mathrm{H}), 7.34-7.27(\mathrm{~m}, 4 \mathrm{H}), 7.20-7.11(\mathrm{~m}, 5 \mathrm{H}), 7.04(\mathrm{dd}, J=$ 8.4, $6.3 \mathrm{~Hz}, 3 \mathrm{H}), 6.84$ (d, $J=7.7 \mathrm{~Hz}, 2 \mathrm{H}), 5.82(\mathrm{br}, 1 \mathrm{H}), 2.81(\mathrm{qd}, J=7.4,4.2 \mathrm{~Hz}, 2 \mathrm{H}), 1.31$ (t, $J=7.6$ $\mathrm{Hz}, 3 \mathrm{H})$.

${ }^{13}$ C NMR (101 MHz, CDCl 3$) \delta 163.0,161.6,152.8,144.1,141.1,140.7,137.8,135.2,133.6,132.0$, $129.7,129.3,129.1,128.6,128.4,128.2,127.0,126.2,124.6,123.9,123.7,123.3,122.8,122.6,116.2$, 116.0, 107.6, 29.3, 15.3.

HRMS (ESI) calculated for $[\mathrm{M}+\mathrm{H}]^{+} \mathrm{C}_{35} \mathrm{H}_{28} \mathrm{NO}_{3}{ }^{+}, \mathrm{m} / \mathrm{z}: 510.2069$, found: 510.2075.

M.P. $110-113^{\circ} \mathrm{C}$.

$[\alpha]^{20}{ }_{\mathrm{D}}=195^{\circ}\left(\mathrm{c}=0.2, \mathrm{CHCl}_{3}\right)$.

HPLC analysis: HPLC DAICEL CHIRALPAK AD-H, hexane/isopropyl alcohol = 90/10, flow rate = 1 $\mathrm{mL} / \mathrm{min}, \lambda=254 \mathrm{~nm})$, tR $($ major $)=18.6 \mathrm{~min}, \mathrm{t}($ minor $)=12.3 \mathrm{~min}$, ee $=96 \%$.

Chiral HPLC spectrum of racemic $\mathbf{6} \boldsymbol{c a}$ 


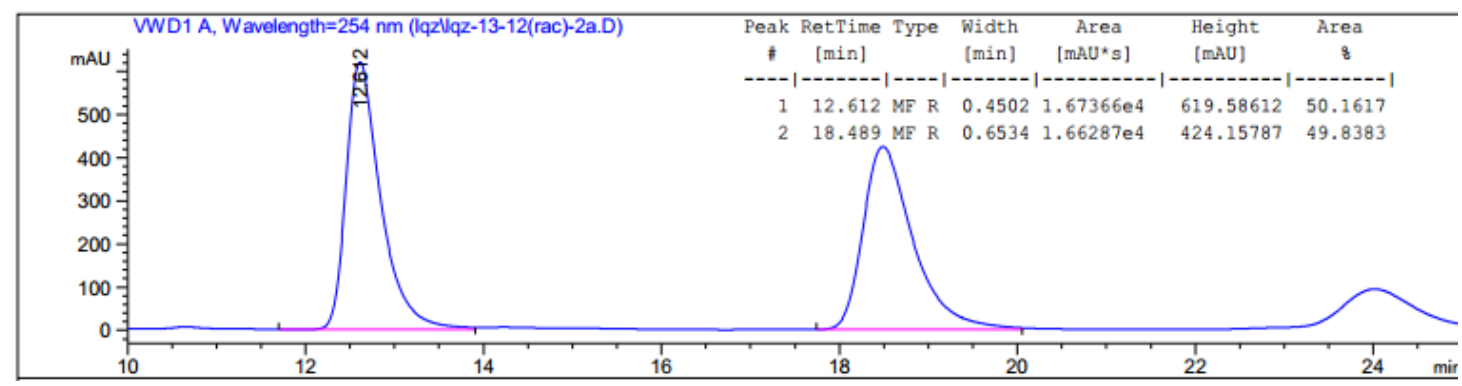

Chiral HPLC spectrum of $\mathbf{6 c a}$
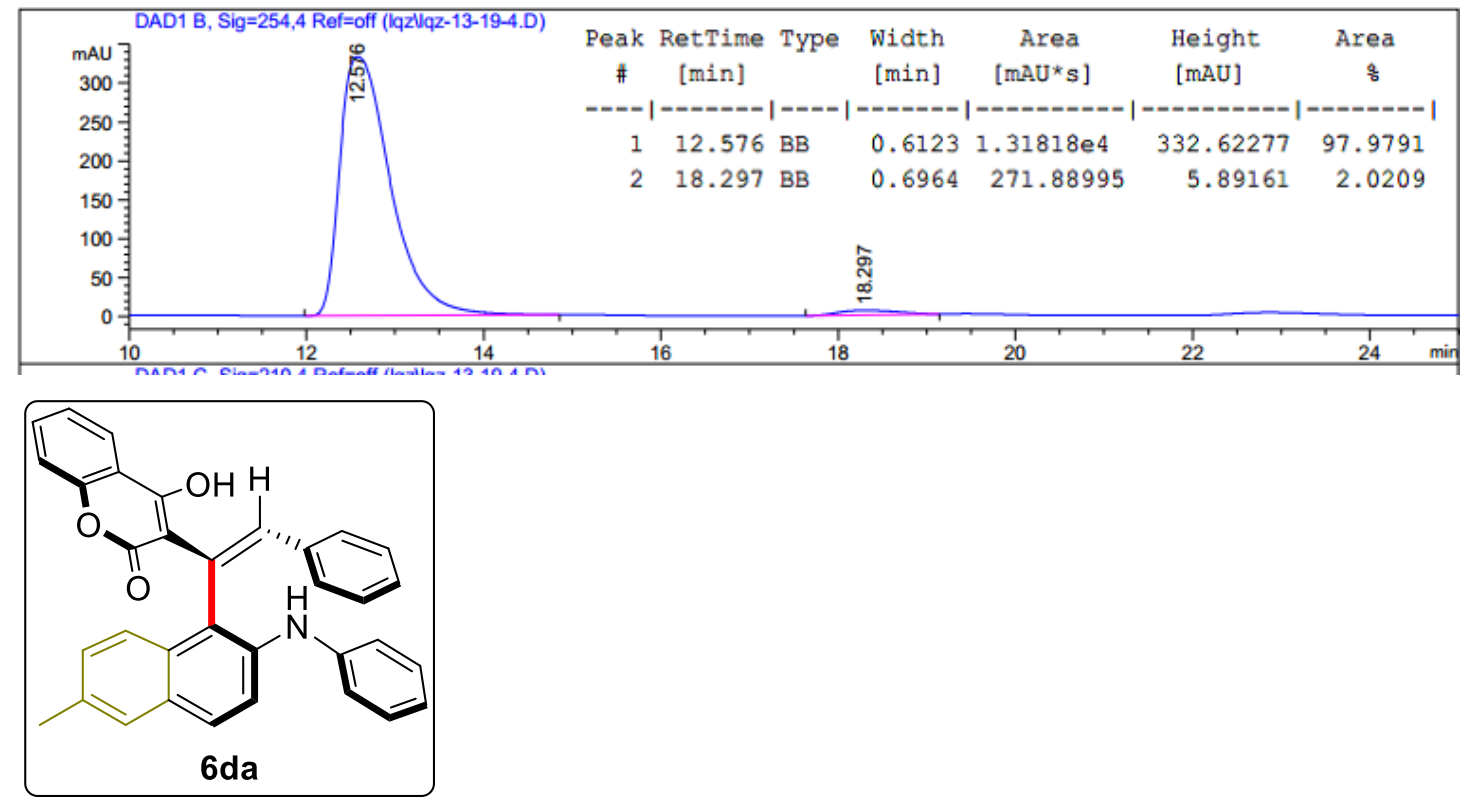

(aS)-(E)-4-hydroxy-3-(1-(6-methyl-2-(phenylamino)naphthalen-1-yl)-2-phenylvinyl)-2Hchromen-2-one (6da)

According to procedure $\mathbf{D}, \mathbf{6 d a}$ was obtained as a yellow solid, $29.0 \mathrm{mg}$ (59\% yield, $93 \%$ ee).

${ }^{1} \mathbf{H}$ NMR (400 MHz, CDCl$) \delta 10.62(\mathrm{br}, 1 \mathrm{H}), 8.32$ (d, $\left.J=8.6 \mathrm{~Hz}, 1 \mathrm{H}\right), 7.77$ (d, $\left.J=7.9 \mathrm{~Hz}, 1 \mathrm{H}\right), 7.64$ $(\mathrm{d}, J=9.0 \mathrm{~Hz}, 1 \mathrm{H}), 7.55(\mathrm{~s}, 1 \mathrm{H}), 7.49-7.44(\mathrm{~m}, 1 \mathrm{H}), 7.39(\mathrm{~s}, 1 \mathrm{H}), 7.35$ (d, J=5.0 Hz, 1H), $7.33(\mathrm{~s}, 1 \mathrm{H})$, $7.31-7.25(\mathrm{~m}, 2 \mathrm{H}), 7.19-7.08(\mathrm{~m}, 6 \mathrm{H}), 7.01(\mathrm{~d}, J=7.7 \mathrm{~Hz}, 2 \mathrm{H}), 6.85$ (d, J= 7.8 Hz, 2H), 5.79 (br, $1 \mathrm{H}), 2.46(\mathrm{~s}, 3 \mathrm{H})$.

${ }^{13}$ C NMR (101 MHz, CDCl 3 ) $\delta$ 163.0, 161.7, 152.7, 141.1, 140.7, 137.0, 135.1, 134.7, 132.0, 131.5, $131.0,130.2$, 129.7, 128.9, 128.6, 128.3, 127.3, 126.8, 125.4, 124.5, 123.9, 123.7, 123.3, 122.5, 117.0, 116.23, 116.21, 107.6, 21.5.

HRMS (ESI) calculated for $[\mathrm{M}+\mathrm{H}]^{+} \mathrm{C}_{34} \mathrm{H}_{26} \mathrm{NO}_{3}{ }^{+}, \mathrm{m} / \mathrm{z}: 496.1913$, found: 496.1917.

M.P. $125-127^{\circ} \mathrm{C}$.

$[\alpha]^{20}{ }_{\mathrm{D}}=105^{\circ}\left(\mathrm{c}=0.2, \mathrm{CHCl}_{3}\right)$.

HPLC analysis: HPLC DAICEL CHIRALPAK AD-H, hexane/isopropyl alcohol = 60/40, flow rate $=1$ $\mathrm{mL} / \mathrm{min}, \lambda=254 \mathrm{~nm})$, tR (major) $=24.3 \mathrm{~min}, \mathrm{tR}($ minor $)=7.3 \mathrm{~min}$, ee $=93 \%$.

Chiral HPLC spectrum of racemic $6 \mathbf{d a}$ 


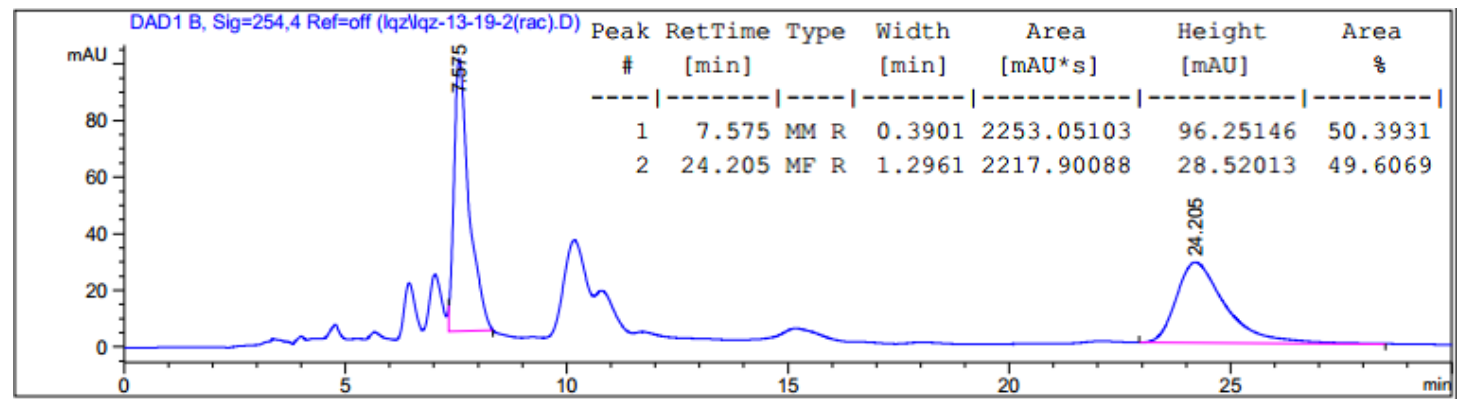

Chiral HPLC spectrum of $\mathbf{6} \mathrm{da}$
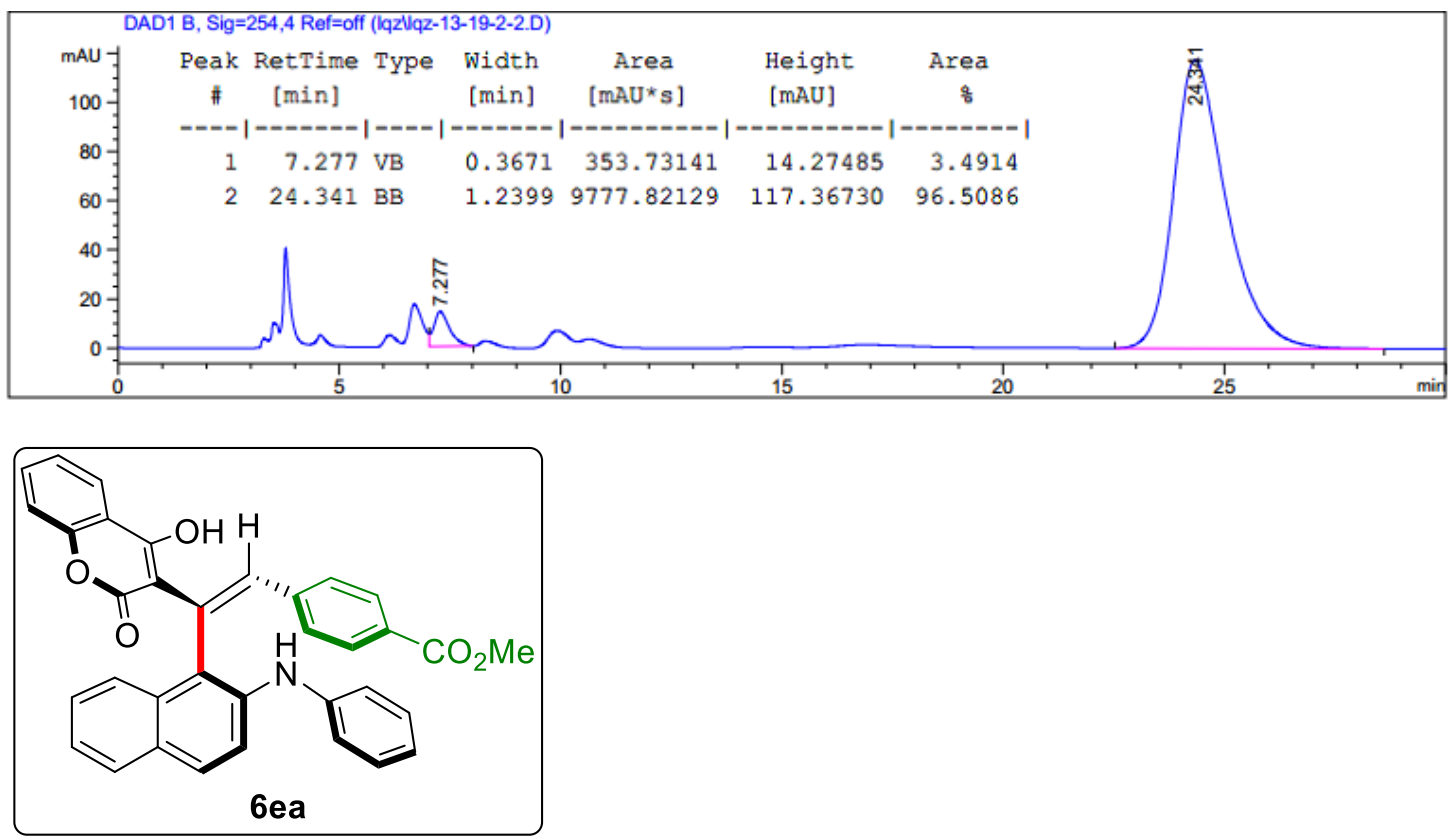

(aS)-(E)-4-hydroxy-3-(1-(6-methyl-2-(phenylamino)naphthalen-1-yl)-2-phenylvinyl)-2Hchromen-2-one (6ea)

According to procedure D, 6ea was obtained as a red-brown solid, $38.0 \mathrm{mg}$ ( $71 \%$ yield, $96 \%$ ee).

${ }^{1}$ H NMR (400 MHz, CDCl $\left.)_{3}\right) 10.56$ (br, 1H), 8.36 (dd, $\left.J=8.4,1.0 \mathrm{~Hz}, 1 \mathrm{H}\right), 7.83-7.80$ (m, 4H), 7.78 $-7.76(\mathrm{~m}, 1 \mathrm{H}), 7.58-7.48(\mathrm{~m}, 3 \mathrm{H}), 7.43(\mathrm{ddd}, J=8.1,6.9,1.2 \mathrm{~Hz}, 1 \mathrm{H}), 7.38-7.31(\mathrm{~m}, 3 \mathrm{H}), 7.24-$ $7.18(\mathrm{~m}, 2 \mathrm{H}), 7.16(\mathrm{~d}, J=9.0 \mathrm{~Hz}, 1 \mathrm{H}), 7.10-7.08(\mathrm{~m}, 2 \mathrm{H}), 6.91-6.88(\mathrm{~m}, 2 \mathrm{H}), 5.86(\mathrm{br}, 1 \mathrm{H}), 3.88(\mathrm{~s}$, $3 \mathrm{H})$.

${ }^{13}$ C NMR (101 MHz, CDCl $) \delta$ 166.6, 162.9, 161.8, 152.8, 140.3, 139.6, 137.9, 133.1, 132.3, 130.6, $130.0,129.83,129.79,129.76,129.3,128.4,128.3,128.2,125.13,125.11,124.8,124.0,123.8,122.5$, $117.0,116.3,116.1,107.2,52.1$.

HRMS (ESI) calculated for $[\mathrm{M}+\mathrm{H}]^{+} \mathrm{C}_{35} \mathrm{H}_{26} \mathrm{NO}_{5}{ }^{+}, \mathrm{m} / \mathrm{z}$ : 540.1811 , found: 540.1810 .

M.P. $126-128^{\circ} \mathrm{C}$.

$[\alpha]^{20}{ }_{\mathrm{D}}=299^{\circ}\left(\mathrm{c}=0.2, \mathrm{CHCl}_{3}\right)$.

HPLC analysis: HPLC DAICEL CHIRALPAK AD-H, hexane/isopropyl alcohol = 80/20, flow rate = 1 $\mathrm{mL} / \mathrm{min}, \lambda=365 \mathrm{~nm})$, $\mathrm{tr}($ major $)=17.4 \mathrm{~min}, \mathrm{tr}($ minor $)=12.8 \mathrm{~min}$, ee $=96 \%$.

Chiral HPLC spectrum of racemic 6 ea 


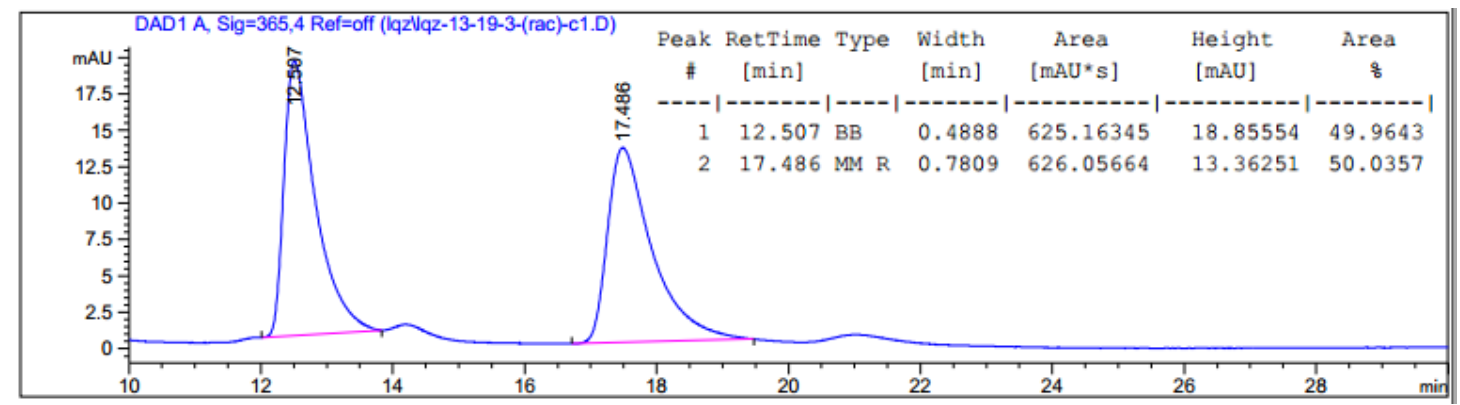

Chiral HPLC spectrum of 6ea
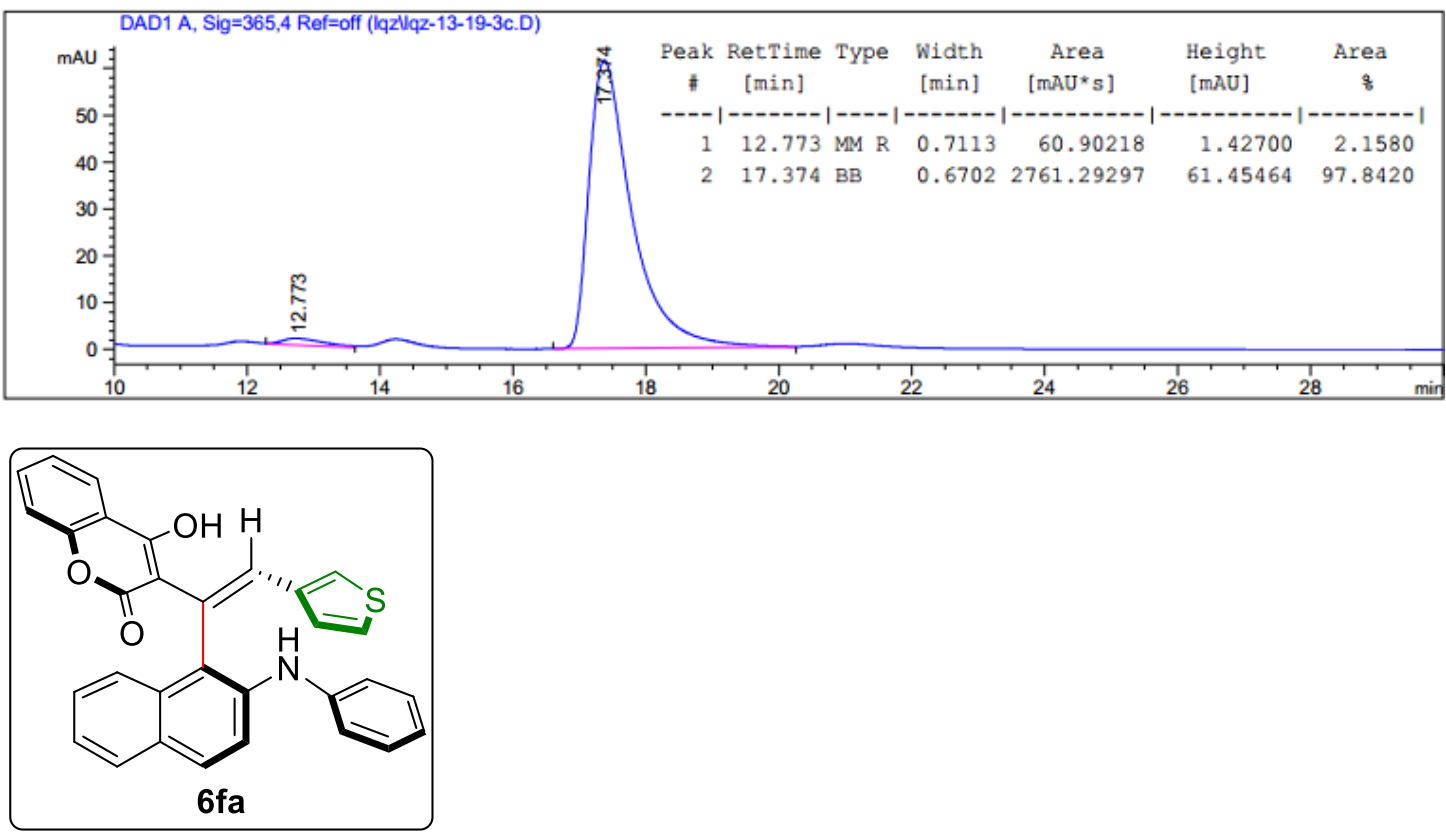

(aS)-(E)-4-hydroxy-3-(1-(2-(phenylamino)naphthalen-1-yl)-2-(thiophen-3-yl)vinyl)-2Hchromen-2-one (6fa)

According to procedure $\mathbf{D}, \mathbf{6 f} \mathbf{a}$ was obtained as a yellow solid, $45.0 \mathrm{mg}$ ( $92 \%$ yield, $92 \%$ ee).

${ }^{1}$ H NMR (400 MHz, CDCl $) \delta 11.12(\mathrm{br}, 1 \mathrm{H}), 8.26(\mathrm{~d}, J=8.5 \mathrm{~Hz}, 1 \mathrm{H}), 7.84-7.77(\mathrm{~m}, 3 \mathrm{H}), 7.66(\mathrm{~s}$, 1H), $7.49-7.44(\mathrm{~m}, 2 \mathrm{H}), 7.42-7.35(\mathrm{~m}, 3 \mathrm{H}), 7.29-7.16(\mathrm{~m}, 4 \mathrm{H}), 7.11-7.08(\mathrm{~m}, 3 \mathrm{H}), 7.02(\mathrm{~d}, J=5.1$ $\mathrm{Hz}, 1 \mathrm{H}), 6.92(\mathrm{dd}, J=5.1,3.6 \mathrm{~Hz}, 1 \mathrm{H}), 6.14(\mathrm{br}, 1 \mathrm{H})$.

${ }^{13} \mathbf{C}$ NMR (101 MHz, $\left.\mathbf{C D C l}_{3}\right) \delta 163.1,162.1,152.7,140.4,139.8,138.0,134.6,132.9,132.1,131.2$, $130.9,130.4,129.8,128.23,128.18,128.0,126.7,125.11,125.06,125.0,124.0,123.7,123.2,122.8$, $122.5,117.3,116.4,116.3,106.6$.

HRMS (ESI) calculated for $[\mathrm{M}+\mathrm{H}]^{+} \mathrm{C}_{31} \mathrm{H}_{22} \mathrm{NO}_{3} \mathrm{~S}^{+}$, m/z: 488.1320 , found: 488.1320 .

M.P. $133-136^{\circ} \mathrm{C}$.

$[\alpha]^{20}{ }_{D}=143^{\circ}\left(\mathrm{c}=0.2, \mathrm{CHCl}_{3}\right)$.

HPLC analysis: HPLC DAICEL CHIRALPAK AD-H, hexane/isopropyl alcohol $=60 / 40$, flow rate $=1$ $\mathrm{mL} / \mathrm{min}, \lambda=254 \mathrm{~nm}$ ), $\mathrm{tR}$ (major) $=10.3 \mathrm{~min}, \mathrm{tr}(\operatorname{minor})=8.8 \mathrm{~min}$, ee $=92 \%$.

Chiral HPLC spectrum of racemic $6 \mathrm{fa}$ 


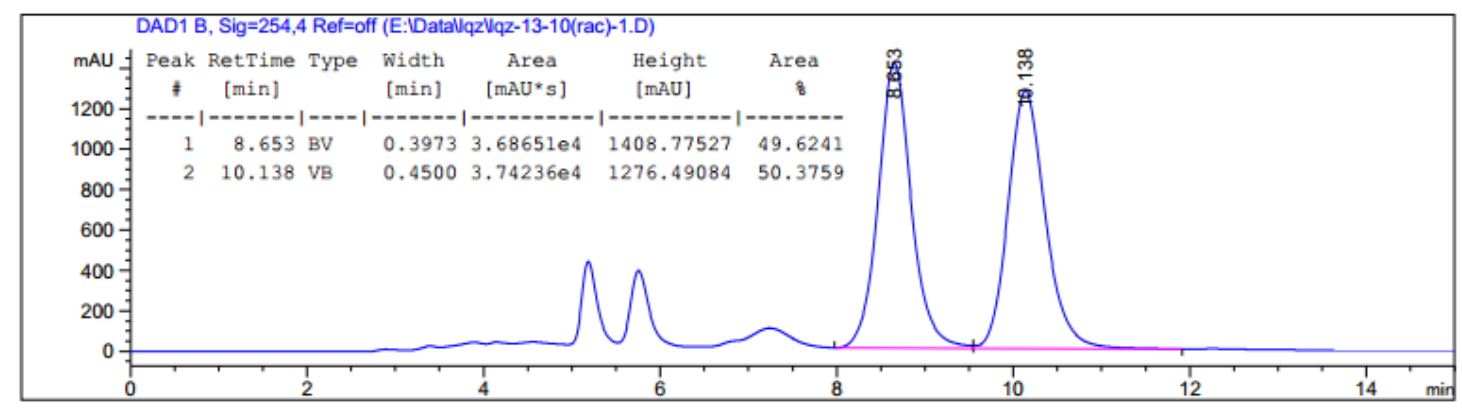

Chiral HPLC spectrum of $\mathbf{6 f a}$

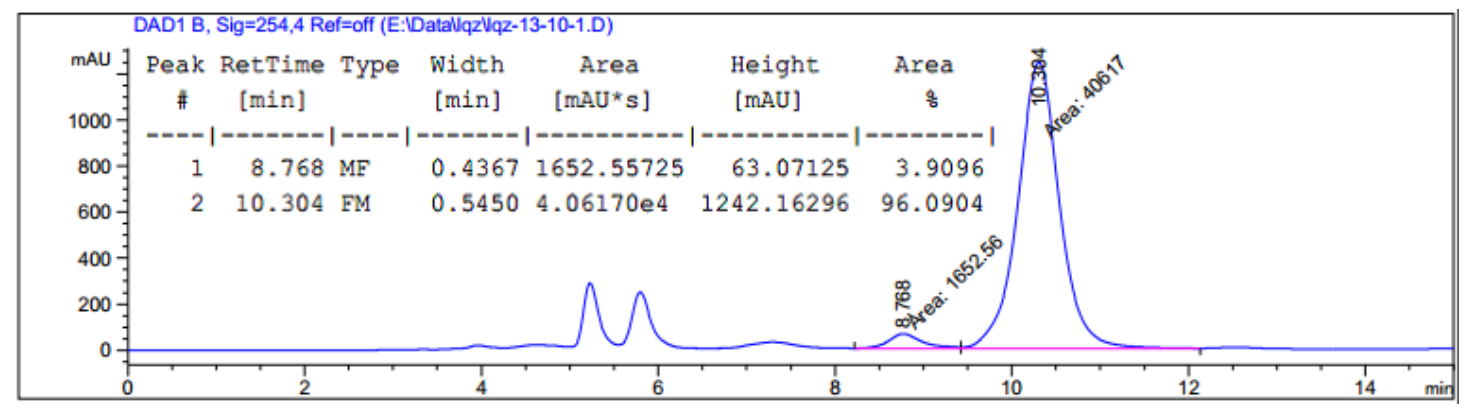




\section{Experimental Procedures for the Transformation of 4xa.}

Procedure C (Experimental procedure for the synthesis of Chiral Binary Naphthalene Squaramides Organocatalysts)

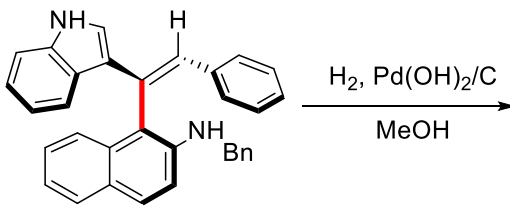

4xa, $93 \%$ ee

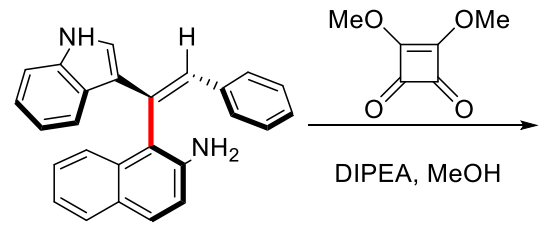

$7 a$

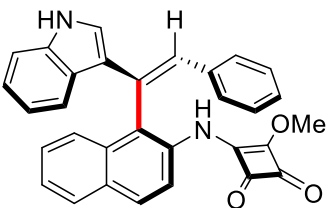

7b, two steps $86 \%$ yield, $93 \%$ ee

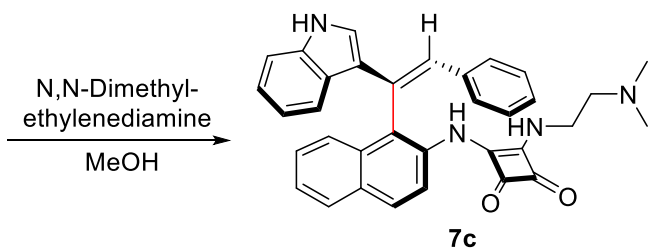

$95 \%$ yield, $93 \%$ ee
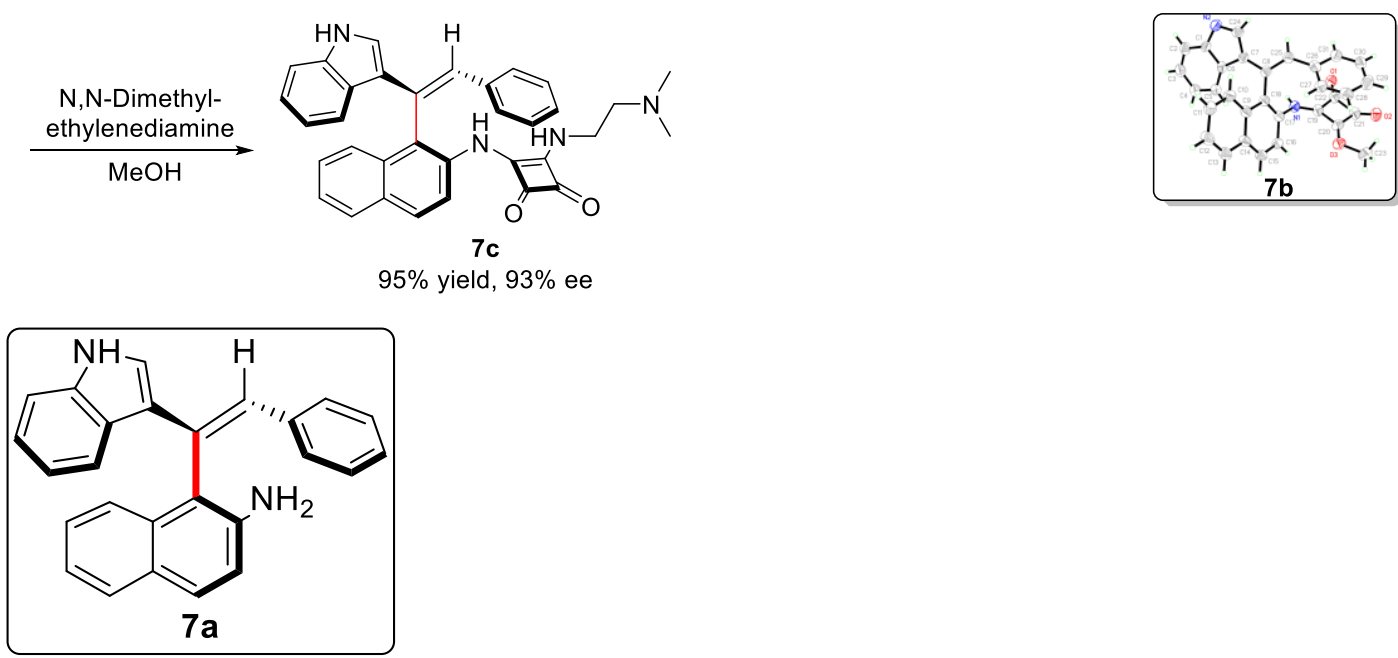

(aS)-(Z)-1-(1-(1H-indol-3-yl)-2-phenylvinyl)naphthalen-2-amine (7a)

4xa $(93 \%$ ee, $900 \mathrm{mg}, 2.0 \mathrm{mmol}) \mathrm{Pd}(\mathrm{OH})_{2} / \mathrm{C}(90 \mathrm{mg})$ and $\mathrm{MeOH}(20 \mathrm{~mL})$ was placed in a hydrogenation tube equipped with a stirring bar. The hydrogenation tube was then put into an autoclave. The system was filled and evacuated with hydrogen for 3 times. The autoclave was pressurized with hydrogen to 7.5 bar hydrogen pressure, and the reaction mixture was stirred at $50{ }^{\circ} \mathrm{C}$ for $24 \mathrm{~h}$. After the completion of the reaction, the mixture was filtered and the solvent was evaporated to afford the crude product. The residue was purified through flash chromatography on silica gel eluted with PE/EA to afford $684 \mathrm{mg} 7 \mathbf{a}(95 \%$ yield, $93 \%$ ee) as a white foam.

${ }^{1}$ H NMR (400 MHz, CDCl $) \delta 8.11(\mathrm{~d}, J=6.9 \mathrm{~Hz}, 1 \mathrm{H}), 7.77(\mathrm{~s}, 1 \mathrm{H}), 7.72-7.69$ (m, 1H), 7.68 (s, 1H), $7.66-7.63(\mathrm{~m}, 2 \mathrm{H}), 7.56(\mathrm{~s}, 1 \mathrm{H}), 7.23-7.15(\mathrm{~m}, 5 \mathrm{H}), 6.99-6.98(\mathrm{~m}, 5 \mathrm{H}), 6.91(\mathrm{~d}, J=8.7 \mathrm{~Hz}, 1 \mathrm{H})$, $6.42(\mathrm{~d}, J=2.6 \mathrm{~Hz}, 1 \mathrm{H}), 3.80(\mathrm{~s}, 2 \mathrm{H})$.

${ }^{13}$ C NMR (101 MHz, $\left.\mathbf{C D C l}_{3}\right) \delta$ 140.6, 137.5, 137.3, 133.2, 131.4, 128.9, 128.3, 128.2, 128.1, 127.9, $127.5,126.7,126.5,125.5,125.2,124.6,122.5,122.4,120.8,120.6,118.5,118.4,118.3,111.7$.

HRMS (ESI) calculated for $[\mathrm{M}+\mathrm{H}]^{+} \mathrm{C}_{26} \mathrm{H}_{21} \mathrm{~N}_{2}{ }^{+}, \mathrm{m} / \mathrm{z}: 361.1705$, found: 361.1702 .

M.P. $59-61^{\circ} \mathrm{C}$.

$[\alpha]^{20}{ }_{\mathrm{D}}=166^{\circ}\left(\mathrm{c}=0.2, \mathrm{CHCl}_{3}\right)$.

HPLC analysis: HPLC DAICEL CHIRALPAK IF, hexane/isopropyl alcohol $=90 / 10$, flow rate $=0.5$ $\mathrm{mL} / \mathrm{min}, \lambda=360 \mathrm{~nm}), \mathrm{tR}($ major $)=20.7 \mathrm{~min}, \mathrm{tR}($ minor $)=20.1 \mathrm{~min}$, ee $=93 \%$.

Chiral HPLC spectrum of racemic $7 \boldsymbol{a}$ 


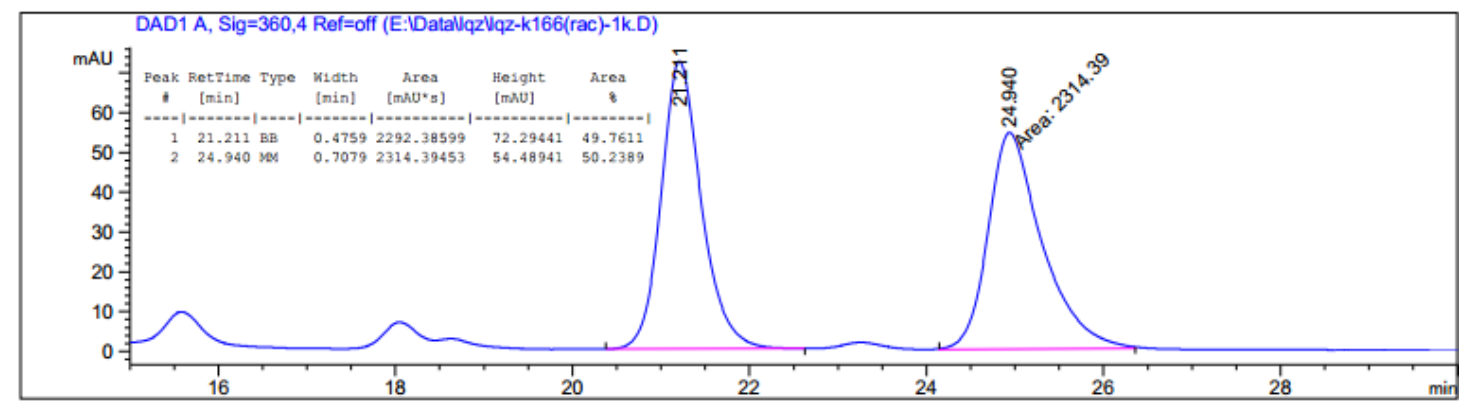

Chiral HPLC spectrum of $7 a$
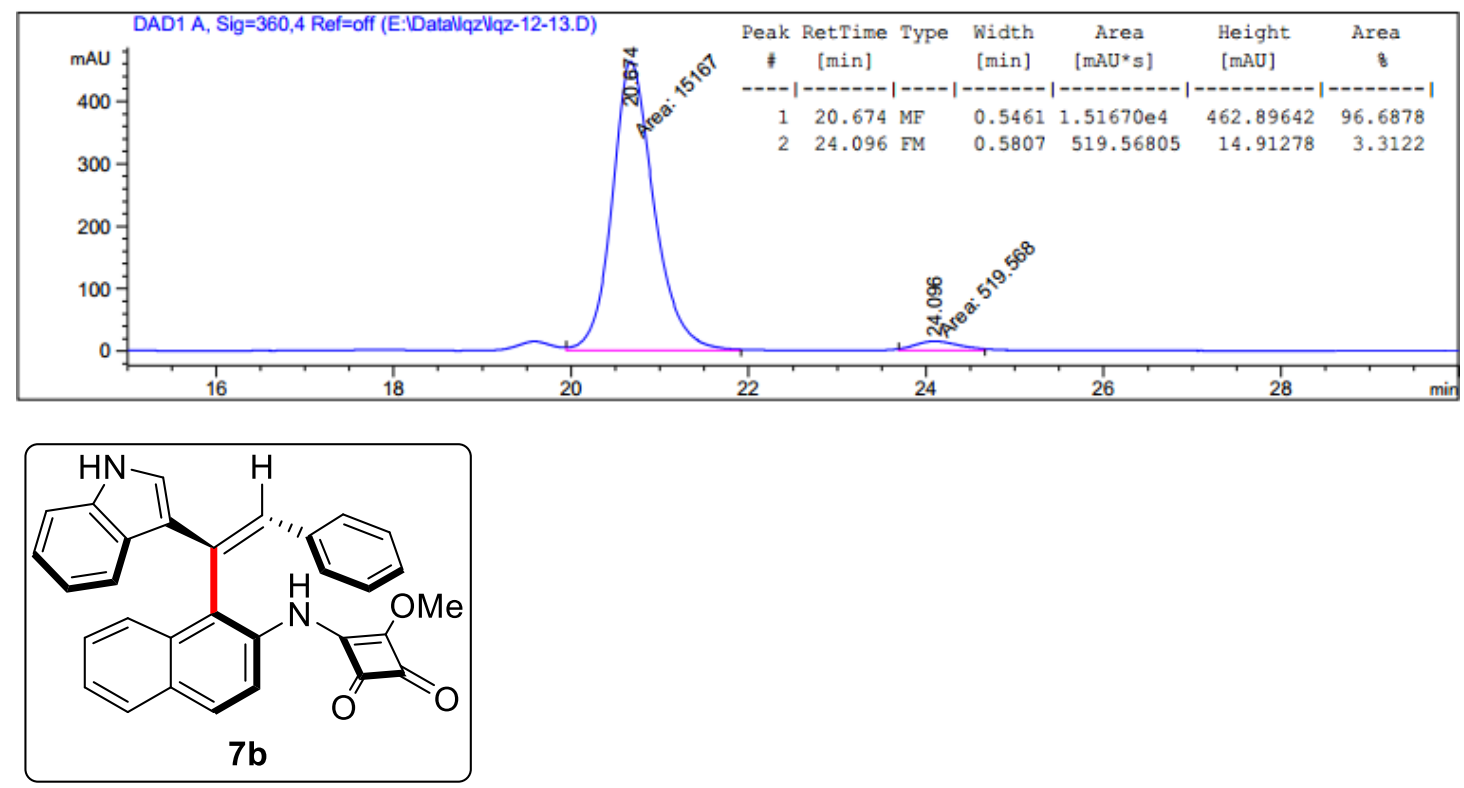

(aS)- (Z)-3-((1-(1-(1H-indol-3-yl)-2-phenylvinyl)naphthalen-2-yl)amino)-4-methoxycyclobut-3ene-1,2-dione $(7 b)$

To a flame-dried round bottom flask equipped with a stir bar, 7a (450 mg, $1.25 \mathrm{mmol})$, 3,4-Dimethoxy3-cyclobutene-1,2-dione (204 mg, $1.4375 \mathrm{mmol})$, DIPEA (103 $\mu \mathrm{L}, 0.625 \mathrm{mmol})$ and $\mathrm{MeOH}(10 \mathrm{~mL})$ was added and the mixture was stirred at $50{ }^{\circ} \mathrm{C}$ for $12 \mathrm{~h}$. After the completion of the reaction, the mixture was filtered and the solid was purified through flash chromatography on silica gel eluted with PE/EA to afford $529 \mathrm{mg} \mathbf{7 b}(90 \%$ yield, $93 \%$ ee) as a white solid.

${ }^{1}$ H NMR (400 MHz, DMSO-d $) \delta 11.18(\mathrm{~d}, J=2.7 \mathrm{~Hz}, 1 \mathrm{H}), 10.40(\mathrm{~s}, 1 \mathrm{H}), 8.02(\mathrm{~d}, J=8.8 \mathrm{~Hz}, 1 \mathrm{H})$, $8.00-7.96(\mathrm{~m}, 1 \mathrm{H}), 7.79(\mathrm{dd}, J=8.4,1.1 \mathrm{~Hz}, 1 \mathrm{H}), 7.74(\mathrm{~d}, J=8.1 \mathrm{~Hz}, 1 \mathrm{H}), 7.54(\mathrm{~s}, 1 \mathrm{H}), 7.49-7.43$ $(\mathrm{m}, 2 \mathrm{H}), 7.42-7.40(\mathrm{~m}, 1 \mathrm{H}), 7.35(\mathrm{ddd}, J=8.3,6.8,1.3 \mathrm{~Hz}, 1 \mathrm{H}), 7.16-7.04(\mathrm{~m}, 3 \mathrm{H}), 6.99-6.97$ (m, $2 \mathrm{H}), 6.82-6.80(\mathrm{~m}, 2 \mathrm{H}), 6.74(\mathrm{~d}, J=2.7 \mathrm{~Hz}, 1 \mathrm{H}), 4.10(\mathrm{~d}, J=4.4 \mathrm{~Hz}, 3 \mathrm{H})$.

${ }^{13}$ C NMR (101 MHz, DMSO) $\delta$ 188.5, 184.3, 178.2, 170.8, 137.9, 137.5, 133.0, 132.3, 132.1, 131.0, $130.6,128.7,128.6,128.32,128.28,127.3,126.9,126.6,126.5,126.13,126.11,125.2,124.3,122.0$, $120.6,120.2,117.9,112.5,60.4$.

HRMS (ESI) calculated for $[\mathrm{M}+\mathrm{H}]^{+} \mathrm{C}_{31} \mathrm{H}_{23} \mathrm{~N}_{2} \mathrm{O}_{3}{ }^{+}, \mathrm{m} / \mathrm{z}$ : 471.1709 , found: 471.1705

M.P. $248-251^{\circ} \mathrm{C}$.

$[\alpha]^{20}{ }_{\mathrm{D}}=37^{\circ}(\mathrm{c}=0.2, \mathrm{THF})$.

HPLC analysis: HPLC DAICEL CHIRALPAK AD-H, hexane/isopropyl alcohol $=60 / 40$, flow rate $=1$ $\mathrm{mL} / \mathrm{min}, \lambda=360 \mathrm{~nm}$ ), tR (major) $=6.9 \mathrm{~min}, \mathrm{tR}($ minor $)=5.6 \mathrm{~min}$, ee $=93 \%$.

Chiral HPLC spectrum of racemic $7 \boldsymbol{b}$ 


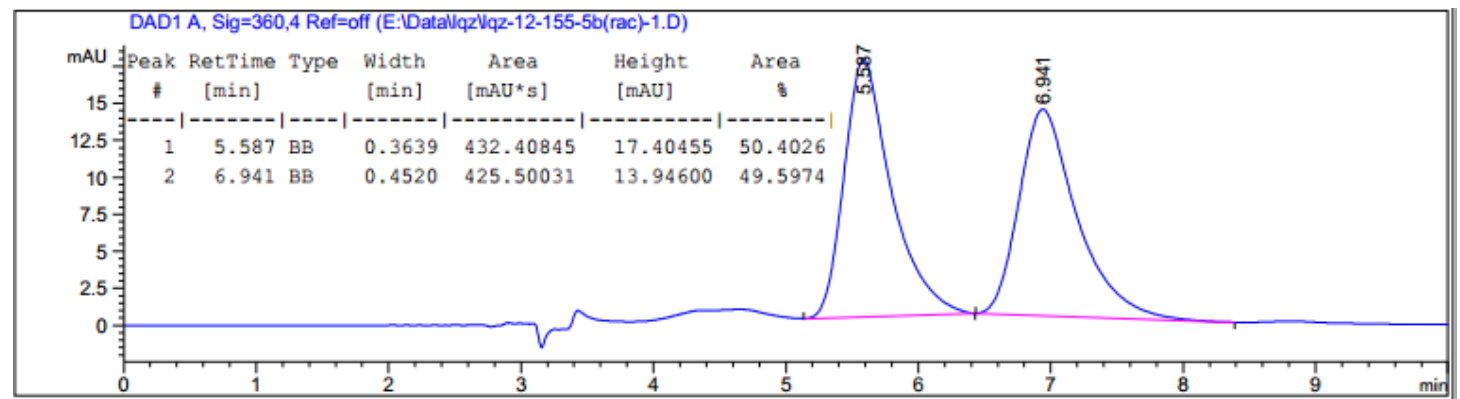

Chiral HPLC spectrum of $\mathbf{7 b}$
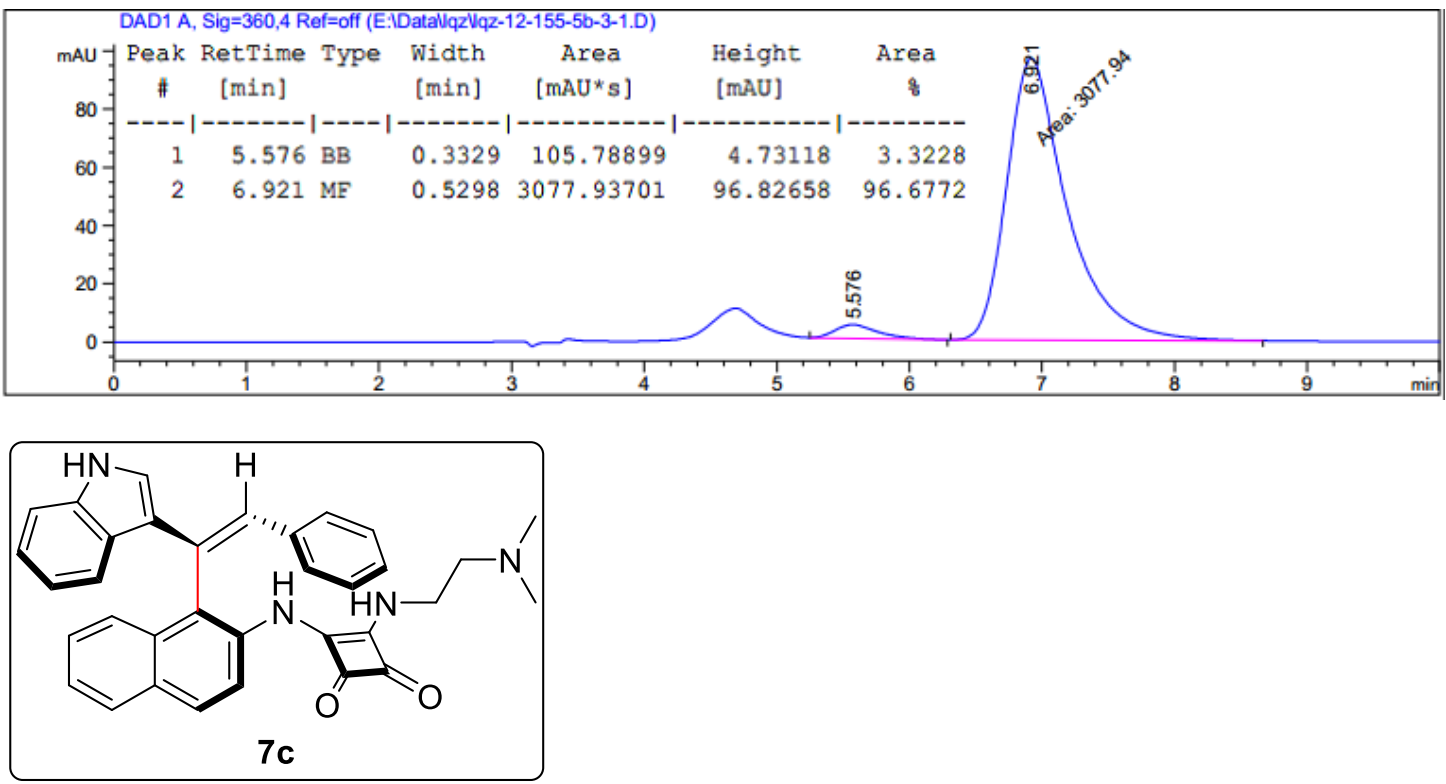

(aS)- (Z)-3-((1-(1-(1H-indol-3-yl)-2-phenylvinyl)naphthalen-2-yl)amino)-4-((2-(dimethylamino) ethyl) amino)cyclobut-3-ene-1,2-dione (7c)

To a flame-dried round bottom flask equipped with a stir bar, $7 \mathbf{b}$ (235 mg, $0.5 \mathrm{mmol}, 93 \%$ ee), N',N'dimethylethane-1,2-diamine (183.1 mg, $0.625 \mathrm{mmol}$ ), DIPEA (26 $\mu \mathrm{L}, 0.25 \mathrm{mmol}$ ) and $\mathrm{MeOH} / \mathrm{CH}_{2} \mathrm{Cl}_{2}$ $(4: 1,5 \mathrm{~mL})$ was added and the mixture was stirred at $50^{\circ} \mathrm{C}$ for $4 \mathrm{~h}$. After the completion of the reaction, the mixture was filtered and the solid was purified through flash chromatography on silica gel eluted with PE/EA to afford $250 \mathrm{mg} 7 \mathbf{c}$ (95\% yield) as a white solid.

${ }^{1}$ H NMR (400 MHz, DMSO-d $) \delta 11.23$ (d, $\left.J=2.7 \mathrm{~Hz}, 1 \mathrm{H}\right), 9.13(\mathrm{~s}, 1 \mathrm{H}), 8.08$ (t, $\left.J=6.0 \mathrm{~Hz}, 1 \mathrm{H}\right), 8.00$ $(\mathrm{d}, J=9.0 \mathrm{~Hz}, 1 \mathrm{H}), 7.93(\mathrm{~d}, J=7.9 \mathrm{~Hz}, 1 \mathrm{H}), 7.85(\mathrm{~d}, J=8.0 \mathrm{~Hz}, 1 \mathrm{H}), 7.76(\mathrm{~d}, J=8.9 \mathrm{~Hz}, 1 \mathrm{H}), 7.72(\mathrm{~d}$, $J=8.5 \mathrm{~Hz}, 1 \mathrm{H}), 7.63(\mathrm{~s}, 1 \mathrm{H}), 7.43-7.40(\mathrm{~m}, 1 \mathrm{H}), 7.38-7.35(\mathrm{~m}, 1 \mathrm{H}), 7.29(\mathrm{ddd}, J=8.3,6.8,1.4 \mathrm{~Hz}$, 1H), 7.12 (dddd, $J=27.6,8.2,7.0,1.2 \mathrm{~Hz}, 2 \mathrm{H}), 7.00-6.98(\mathrm{~m}, 2 \mathrm{H}), 6.94-6.92(\mathrm{~m}, 2 \mathrm{H}), 6.82(\mathrm{~d}, J=$ $2.7 \mathrm{~Hz}, 1 \mathrm{H}), 3.61$ (p, $J=6.2 \mathrm{~Hz}, 2 \mathrm{H}), 2.37$ (t, $J=5.9 \mathrm{~Hz}, 2 \mathrm{H}), 2.13(\mathrm{~s}, 6 \mathrm{H})$.

${ }^{13}$ C NMR (101 MHz, DMSO) $\delta 185.3,180.6,169.4,164.6,137.74,137.72,134.1,131.9,131.0,130.8$, $128.6,128.52$, 128.47, 128.2, 127.2, 127.0, 126.9, 126.6, 126.2, 125.4, 125.3, 125.2, 122.9, 122.2, 120.6, $120.4,117.5,112.6,59.7,45.5,41.5$.

HRMS (ESI) calculated for $[\mathrm{M}+\mathrm{H}]^{+} \mathrm{C}_{34} \mathrm{H}_{31} \mathrm{~N}_{4} \mathrm{O}_{2}{ }^{+}, \mathrm{m} / \mathrm{z}: 527.2447$, found: 527.2445.

M.P. $270-272^{\circ} \mathrm{C}$.

$[\alpha]^{20}{ }_{D}=-204^{\circ}(c=0.2, T H F)$. 


\section{X-Ray Structure and Crystal Data of 7b}<smiles>COc1c(Nc2ccc3ccccc3c2/C(=C\c2c[nH]c3ccccc23)c2ccccc2)c2oc1oc2=O</smiles>

$7 \mathrm{~b}$

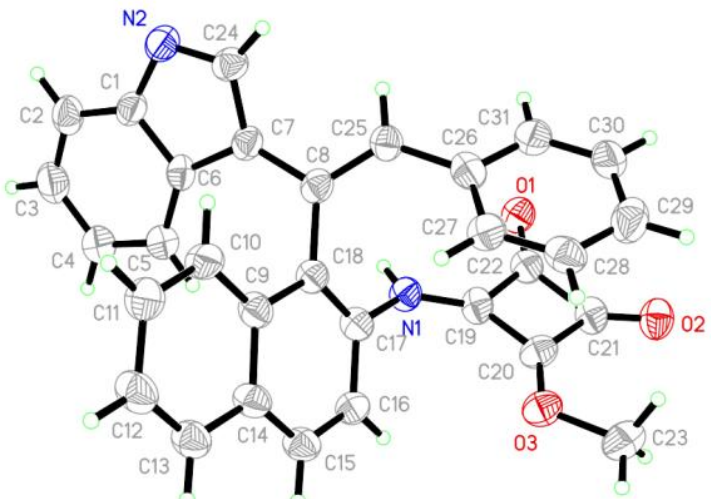

\begin{tabular}{|c|c|c|}
\hline Identification code & \multicolumn{2}{|l|}{ ta_a } \\
\hline Empirical formula & \multicolumn{2}{|l|}{$\mathrm{C}_{31} \mathrm{H}_{21} \mathrm{~N}_{2} \mathrm{O}_{3}$} \\
\hline Formula weight & \multicolumn{2}{|l|}{469.50} \\
\hline Temperature & \multicolumn{2}{|l|}{ 297(2) K } \\
\hline Wavelength & \multicolumn{2}{|l|}{$1.54178 \AA$} \\
\hline Crystal system, space group & \multicolumn{2}{|l|}{ Triclinic, $\mathrm{P} 1$} \\
\hline \multirow[t]{3}{*}{ Unit cell dimensions } & $\mathrm{a}=7.2684(7) \AA$ & alpha $=112.08^{\circ}$ \\
\hline & $\mathrm{b}=13.4917(17) \AA$ & beta $=97.729(4)^{\circ}$ \\
\hline & $\mathrm{c}=13.4917(17) \AA$ & gamma $=97.729(4)^{\circ}$. \\
\hline Volume & \multicolumn{2}{|l|}{$1189.9(2) \AA \wedge 3$} \\
\hline Z, Calculated density & \multicolumn{2}{|l|}{$2,1.310 \mathrm{Mg} / \mathrm{m}^{\wedge} 3$} \\
\hline Absorption coefficient & \multicolumn{2}{|l|}{$0.682 \mathrm{~mm}^{\wedge}-1$} \\
\hline $\mathrm{F}(000)$ & \multicolumn{2}{|l|}{490} \\
\hline Crystal size & \multicolumn{2}{|c|}{$0.180 \times 0.160 \times 0.150 \mathrm{~mm}$} \\
\hline Theta range for data collection & \multicolumn{2}{|c|}{3.609 to $66.595^{\circ}$} \\
\hline Limiting indices & \multicolumn{2}{|c|}{$-8<=\mathrm{h}<=6,-15<=\mathrm{k}<=16,-15<=1<=16$} \\
\hline Reflections collected / uniqu & \multicolumn{2}{|c|}{$9603 / 5939[\mathrm{R}(\mathrm{int})=0.0581]$} \\
\hline Completeness to theta $=66.595$ & \multicolumn{2}{|c|}{$96.4 \%$} \\
\hline Refinement method & \multicolumn{2}{|c|}{ Full-matrix least-squares on $\mathrm{F}^{\wedge} 2$} \\
\hline Data / restraints / parameters & \multicolumn{2}{|c|}{$5939 / 3 / 651$} \\
\hline Goodness-of-fit on $\mathrm{F}^{\wedge} 2$ & \multicolumn{2}{|l|}{1.427} \\
\hline Final $R$ indices $[I>2 \operatorname{sigma}(I)]$ & \multicolumn{2}{|c|}{$\mathrm{R} 1=0.1310, \mathrm{wR} 2=0.3309$} \\
\hline $\mathrm{R}$ indices (all data) & \multicolumn{2}{|c|}{$\mathrm{R} 1=0.1619, \mathrm{wR} 2=0.3843$} \\
\hline Absolute structure parameter & \multicolumn{2}{|l|}{$-0.2(4)$} \\
\hline Extinction coefficient & \multicolumn{2}{|l|}{$\mathrm{n} / \mathrm{a}$} \\
\hline Largest diff. peak and hole & \multicolumn{2}{|c|}{0.676 and -0.448 e. $\AA^{\wedge} \wedge-3$} \\
\hline
\end{tabular}




\section{References}

(1) Yong-Bin Wang, Peiyuan Yu, Zhi-Peng Zhou, Jian Zhang, Jun (Joelle) Wang, Shi-Hui Luo, QiangShuai Gu, K. N. Houk, Bin Tan. Nat. Catal., 2019, 2, 504-513.

(2) He-Yuan Bai, Fu-Xin Tan, Tuan-Qing Liu, Guo-Dong Zhu, Jin-Miao Tian, Tong-Mei Ding, Zhi-Min Chen, Shu-Yu Zhang. Nat. Commun. 2019, 10, 3063. 
6. NMR

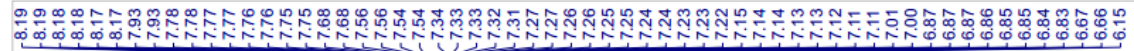

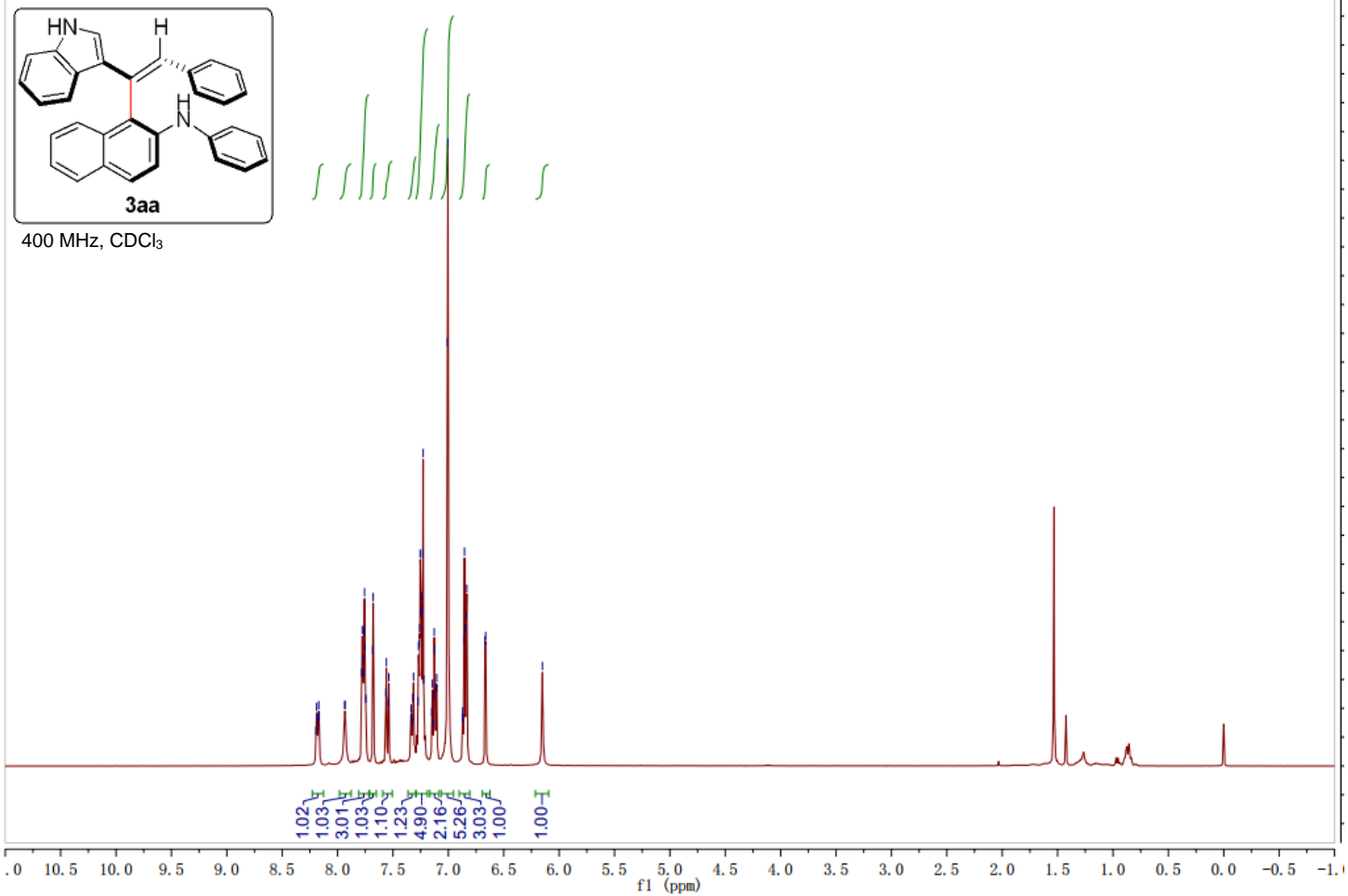

מํ.

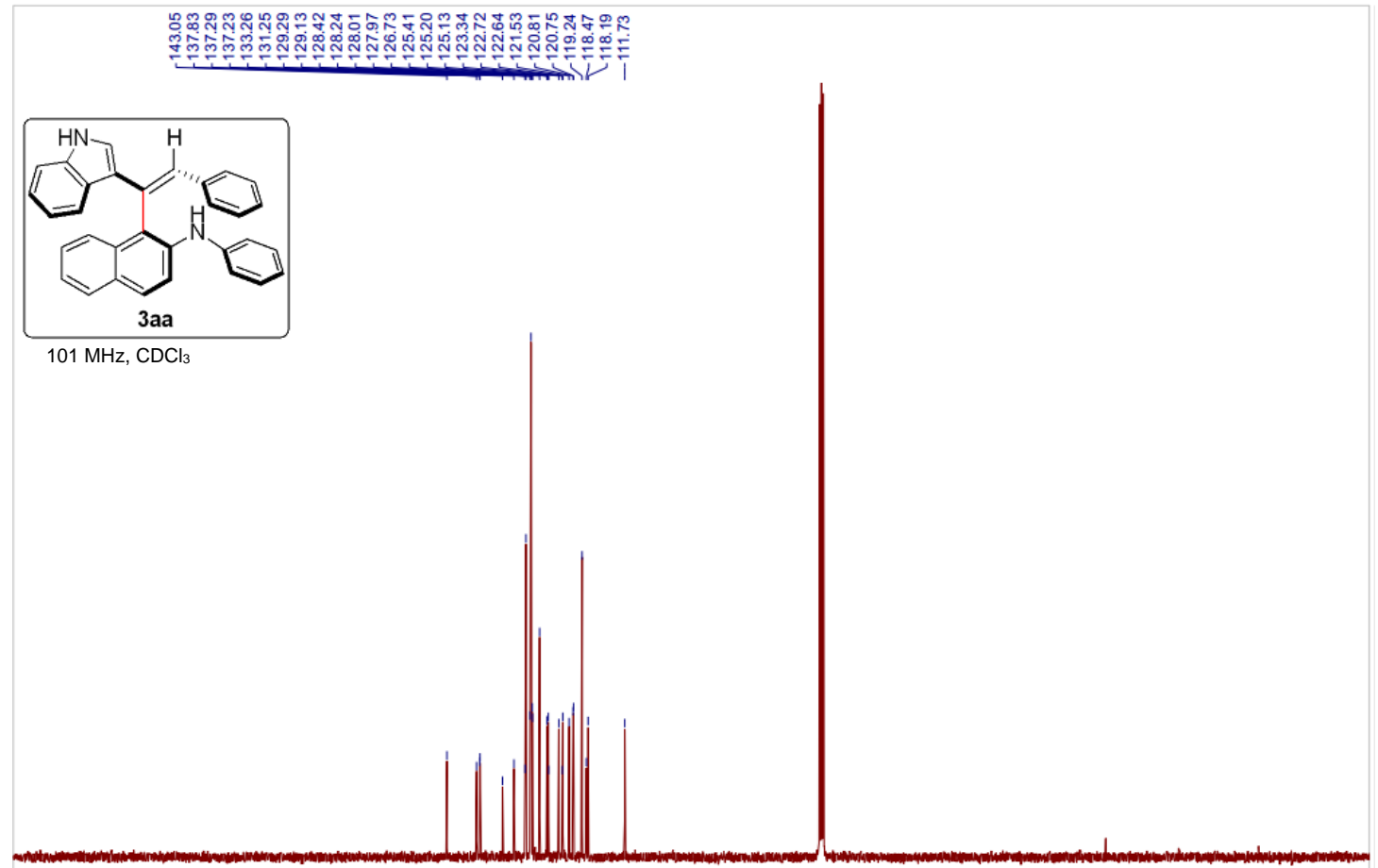

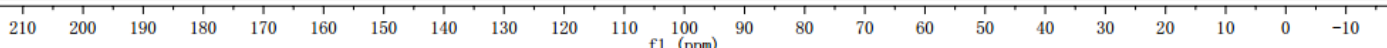



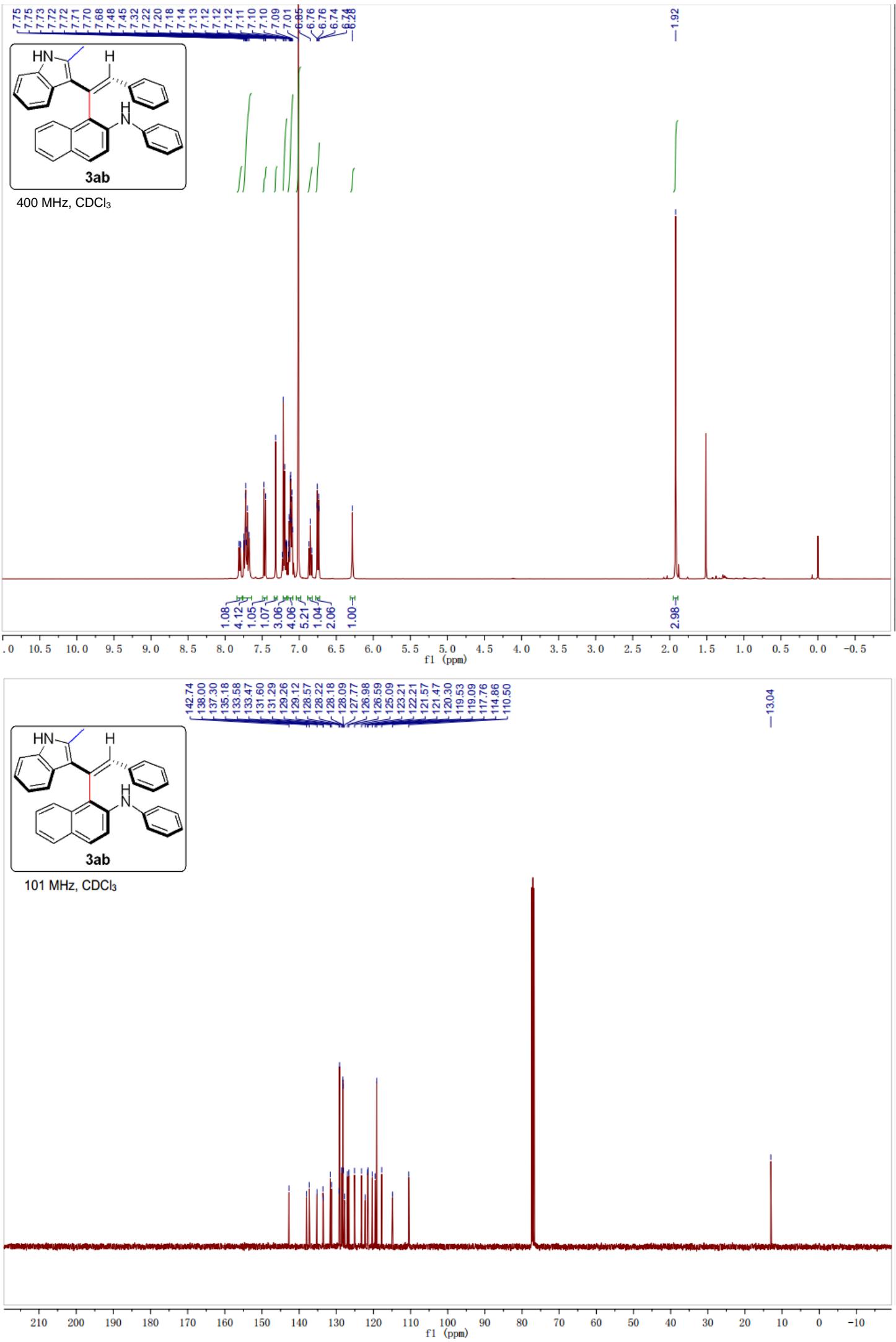


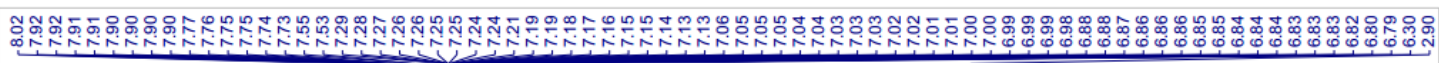

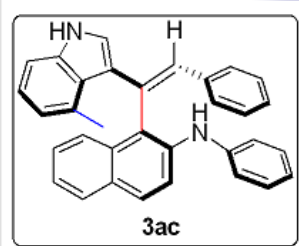

$400 \mathrm{MHz}, \mathrm{CDCl}_{3}$
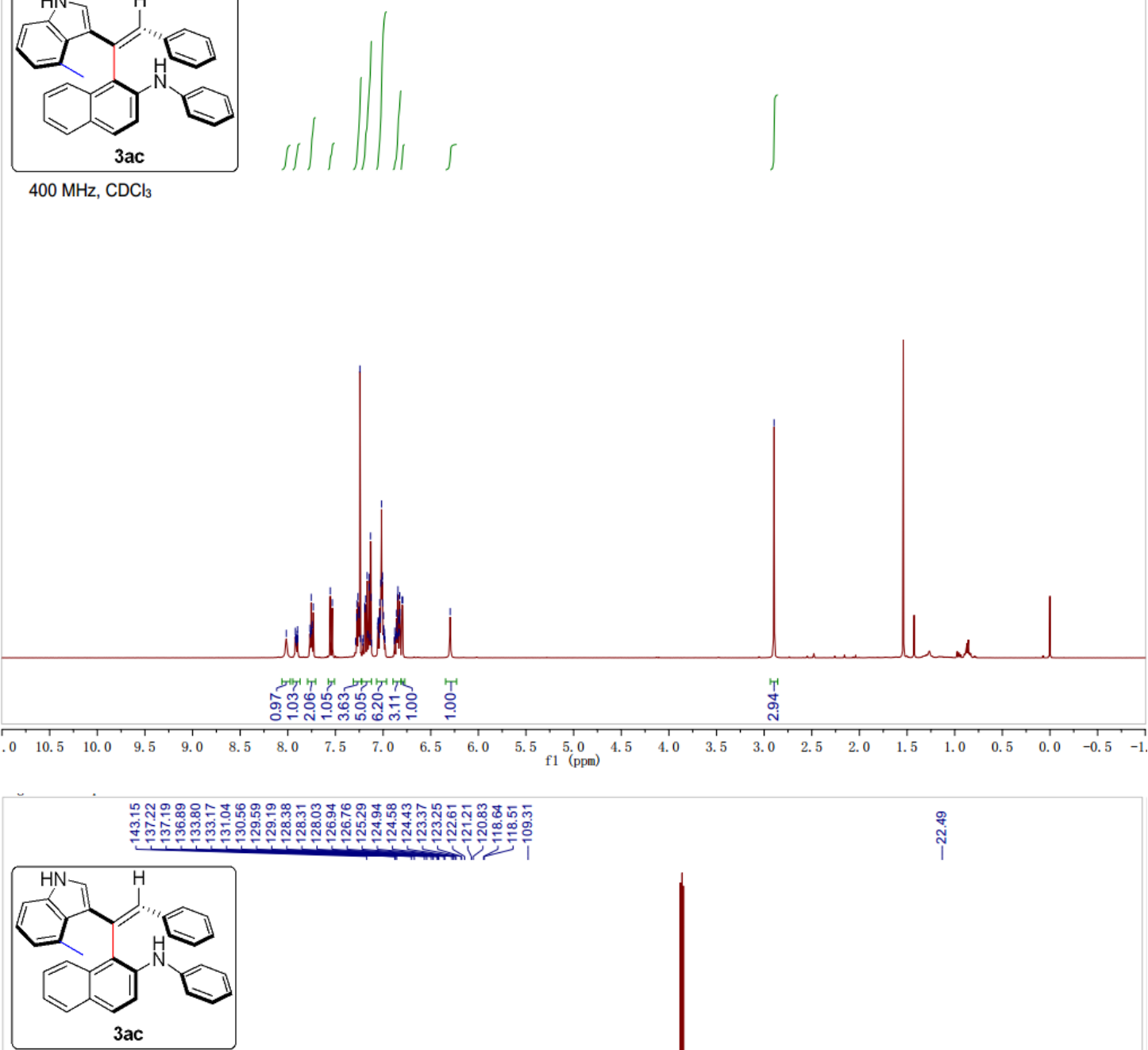

$101 \mathrm{MHz}, \mathrm{CDCl}_{3}$

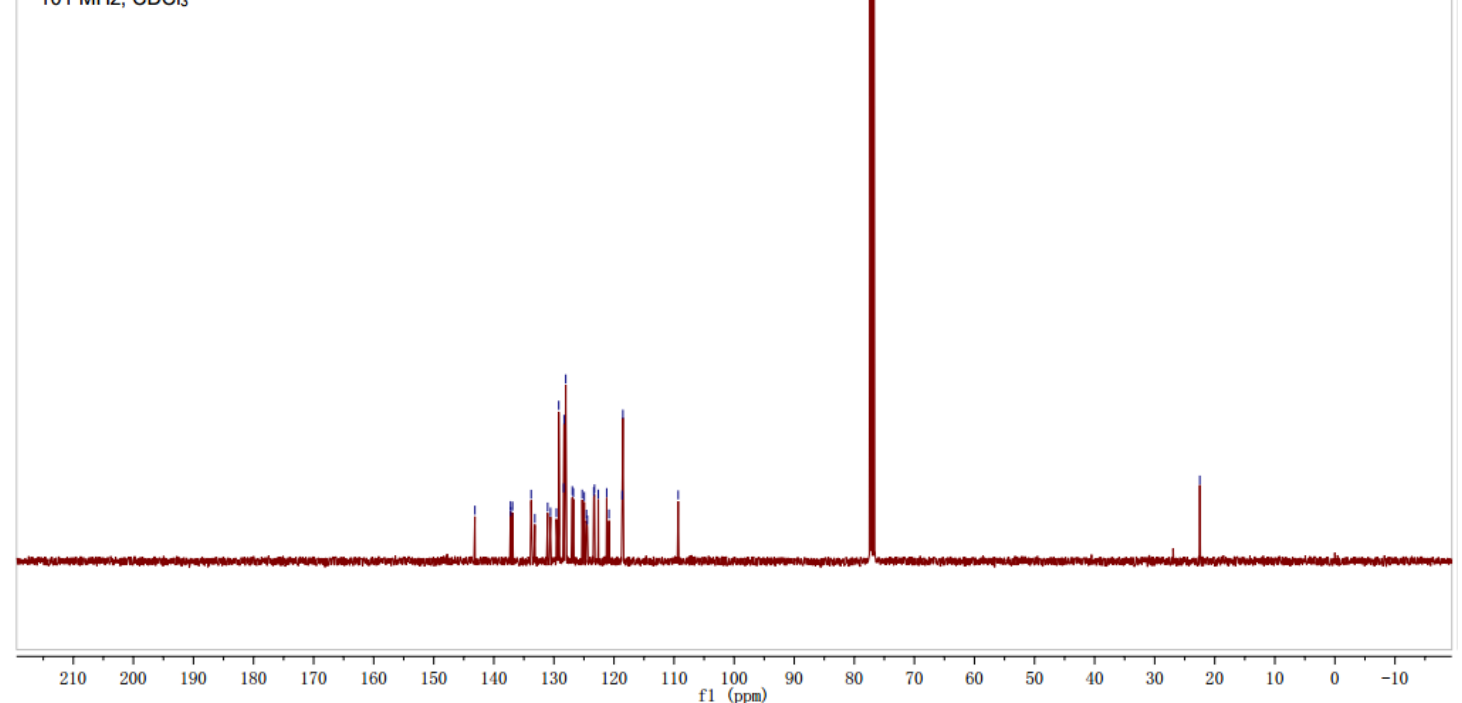




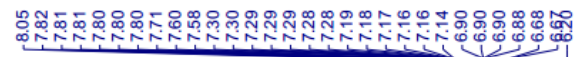

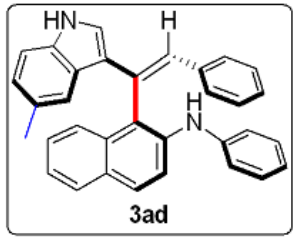

$500 \mathrm{MHz}, \mathrm{CDCl}_{3}$
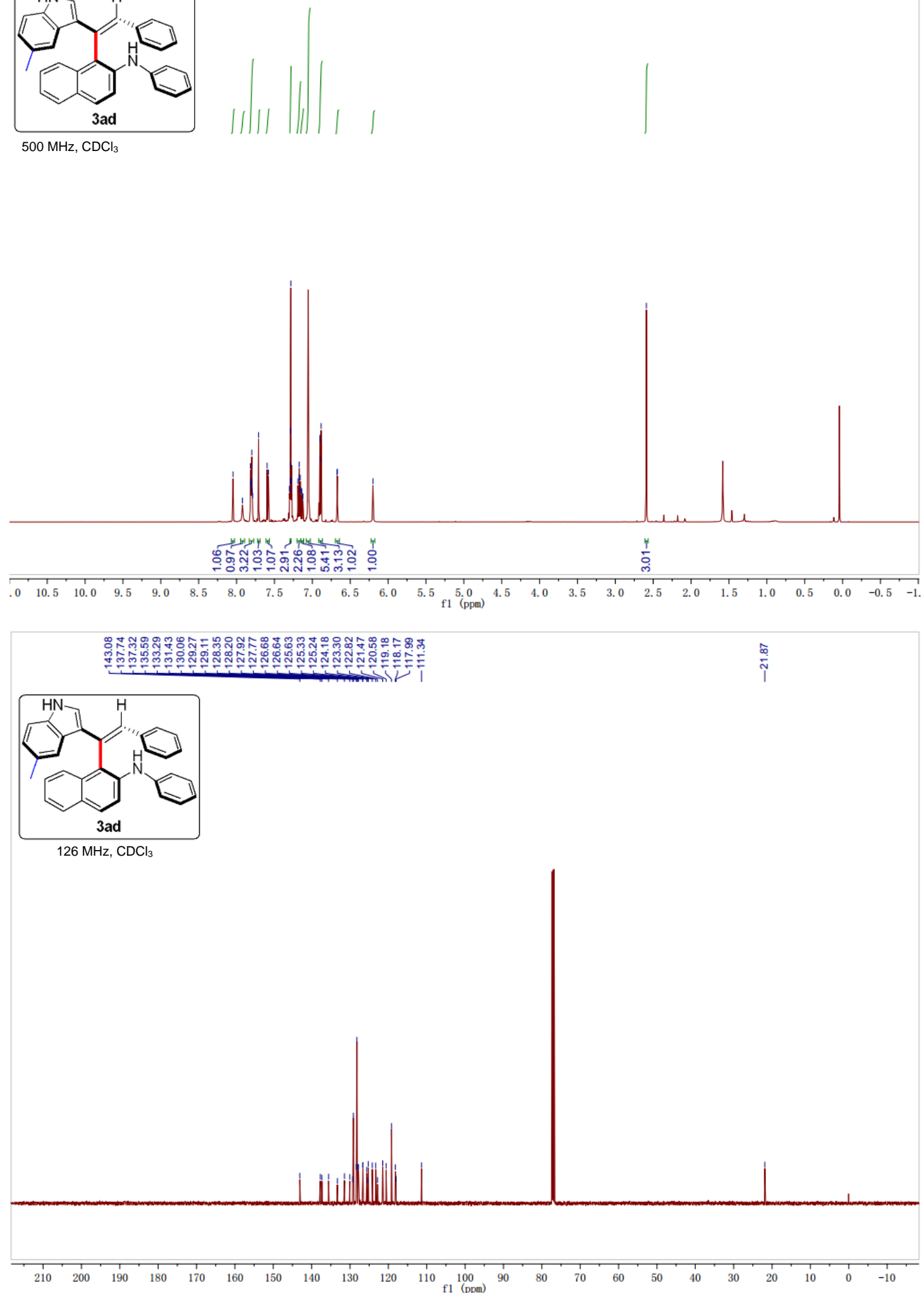


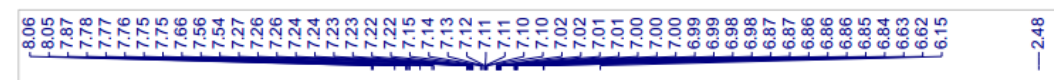

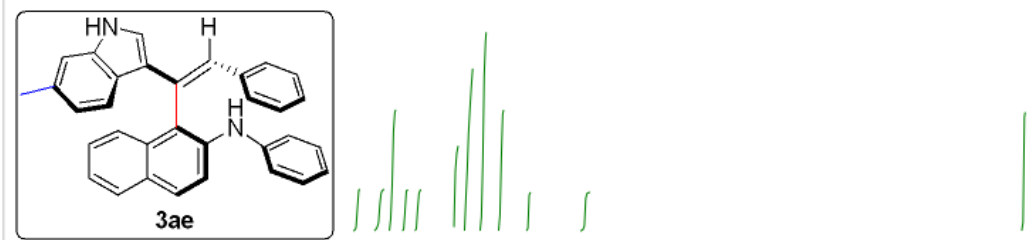

$600 \mathrm{MHz}, \mathrm{CDCl}_{3}$
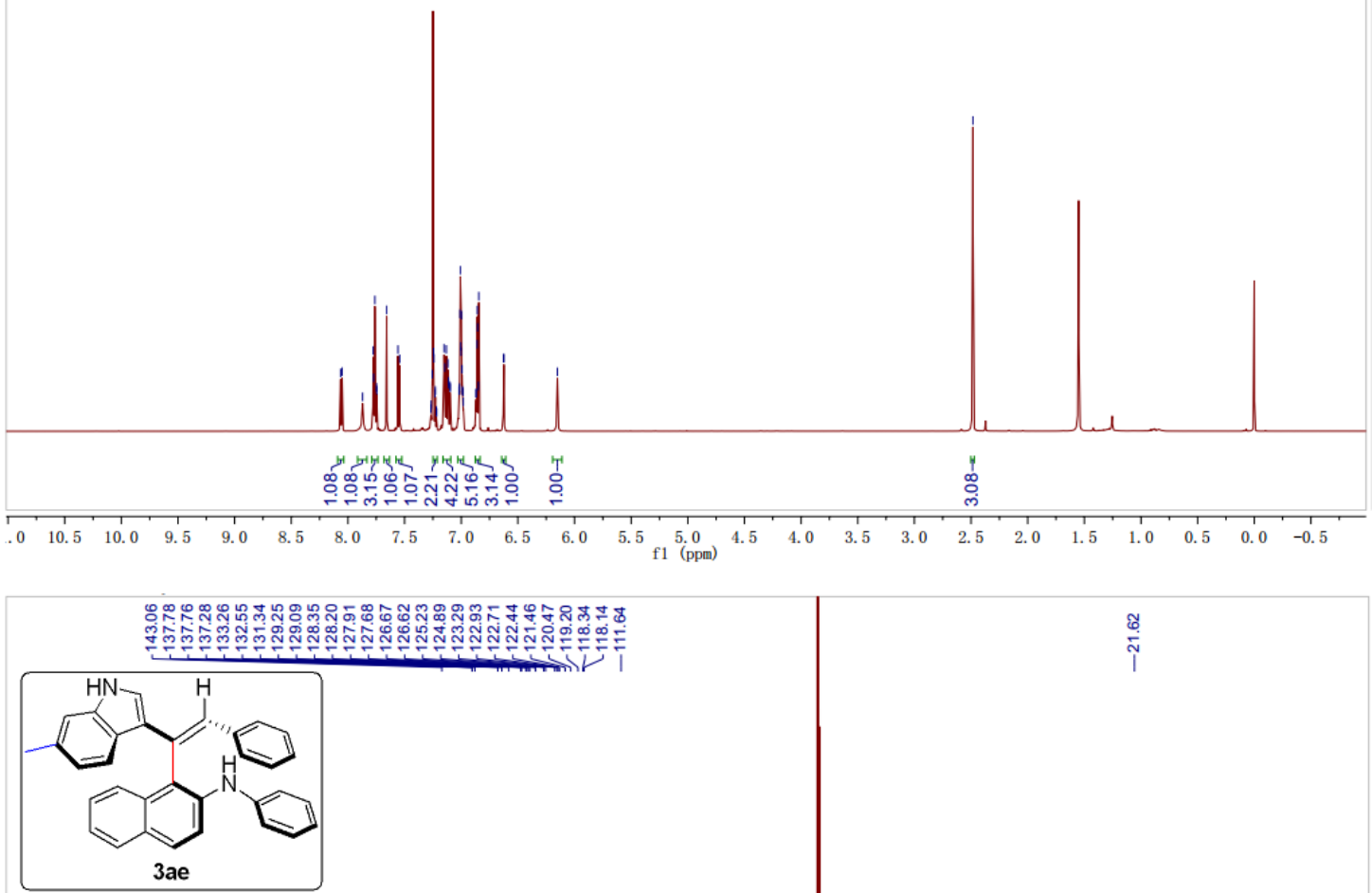

$151 \mathrm{MHz}, \mathrm{CDCl}_{3}$

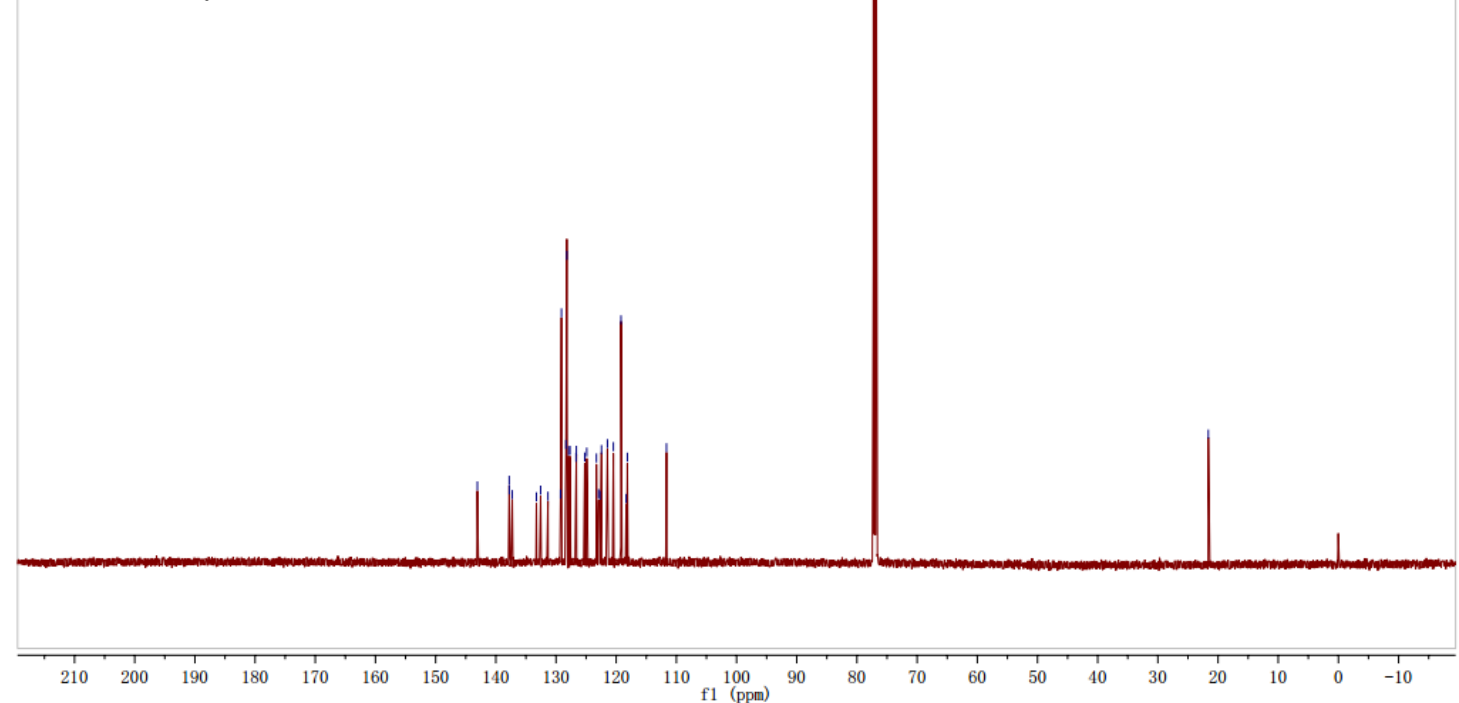



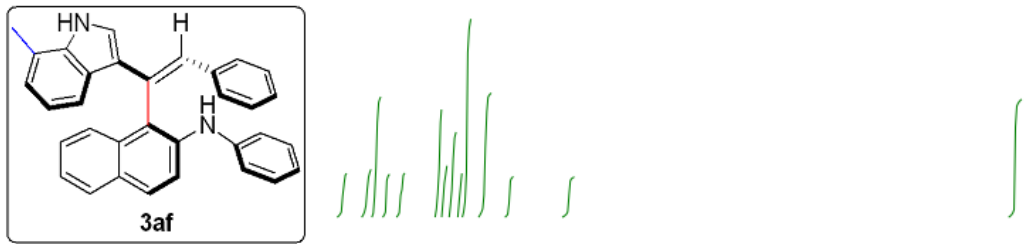

$500 \mathrm{MHz}, \mathrm{CDCl}_{3}$

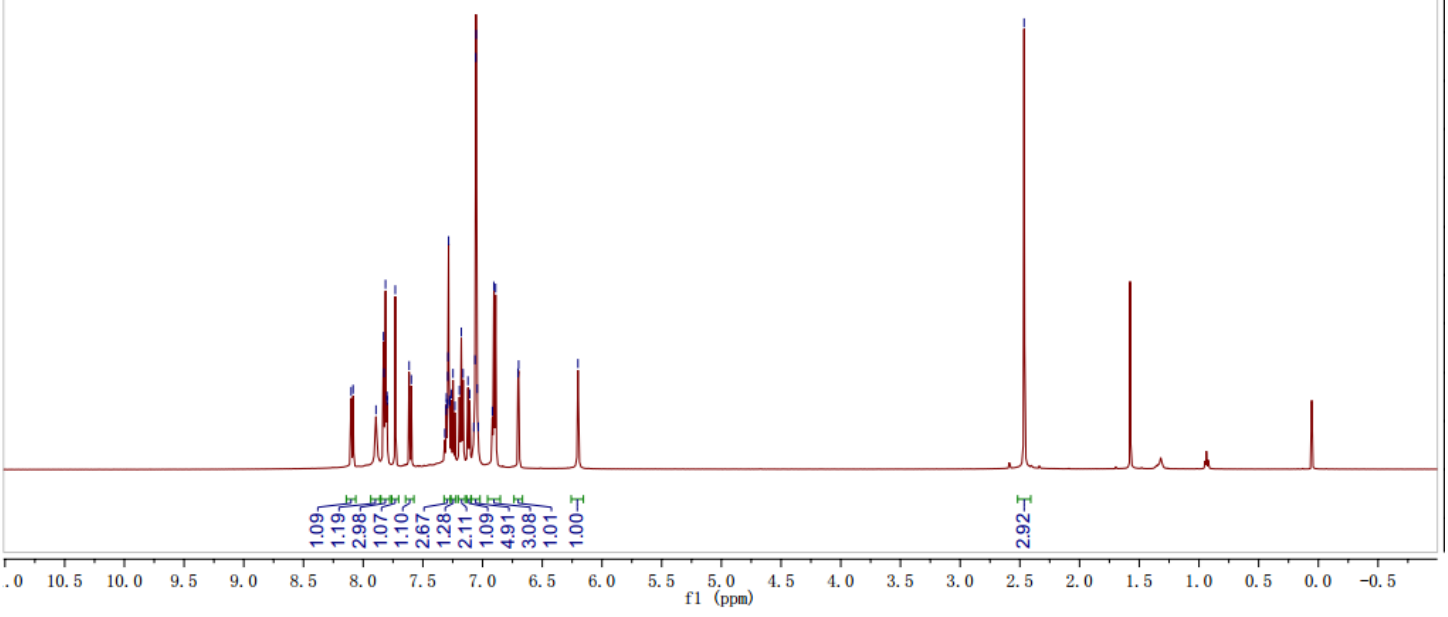

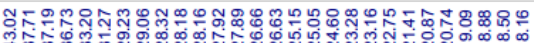

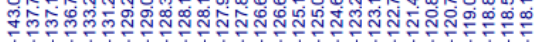

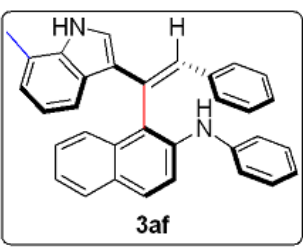

$126 \mathrm{MHz}, \mathrm{CDCl}_{3}$

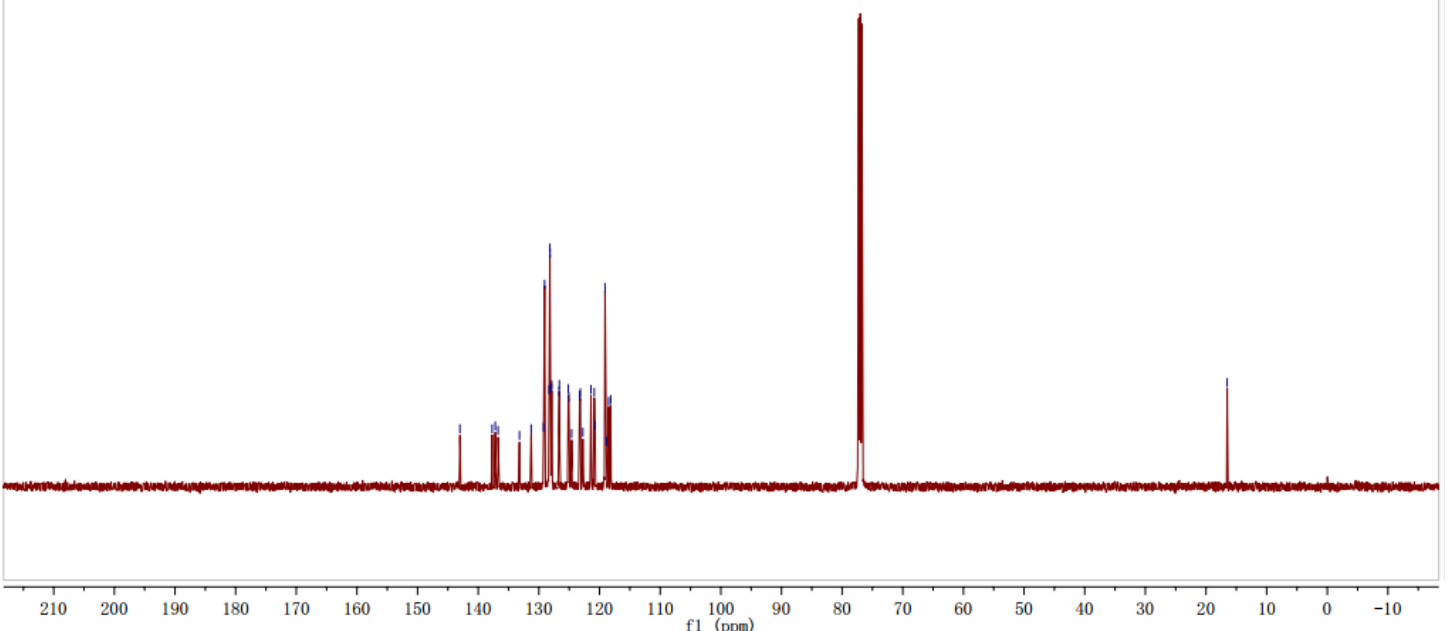




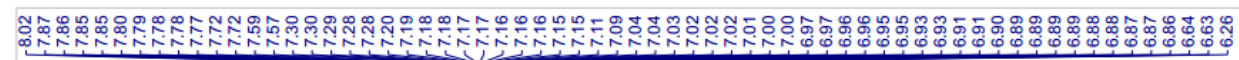

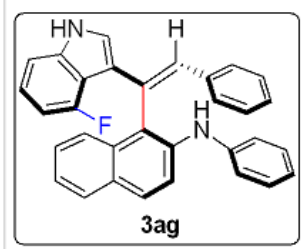

d $\||\||\|\mid\|$

$500 \mathrm{MHz}, \mathrm{CDCl}_{3}$
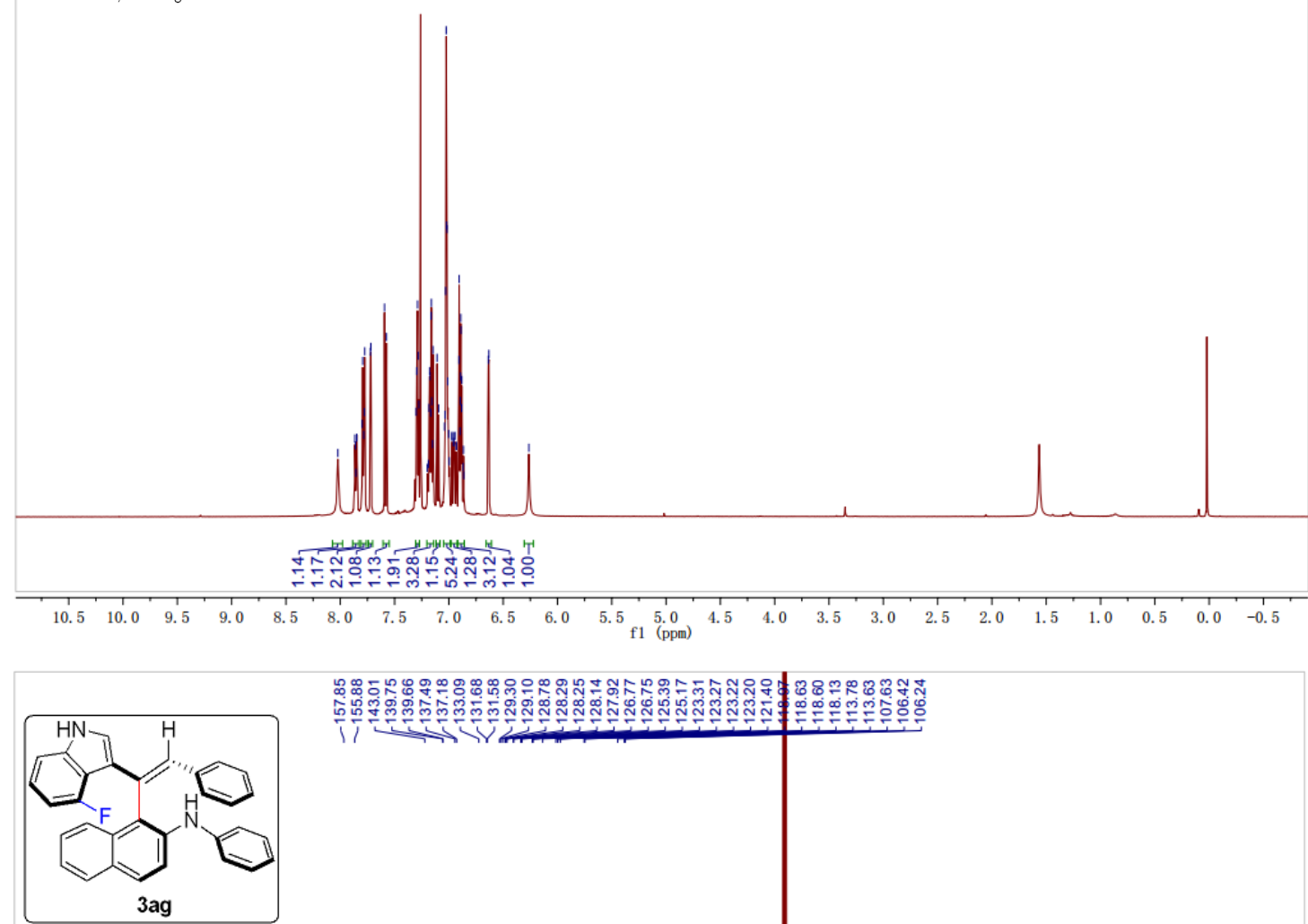

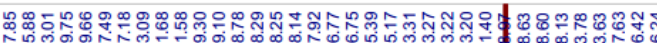

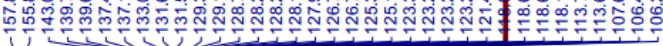

$126 \mathrm{MHz}, \mathrm{CDCl}_{3}$

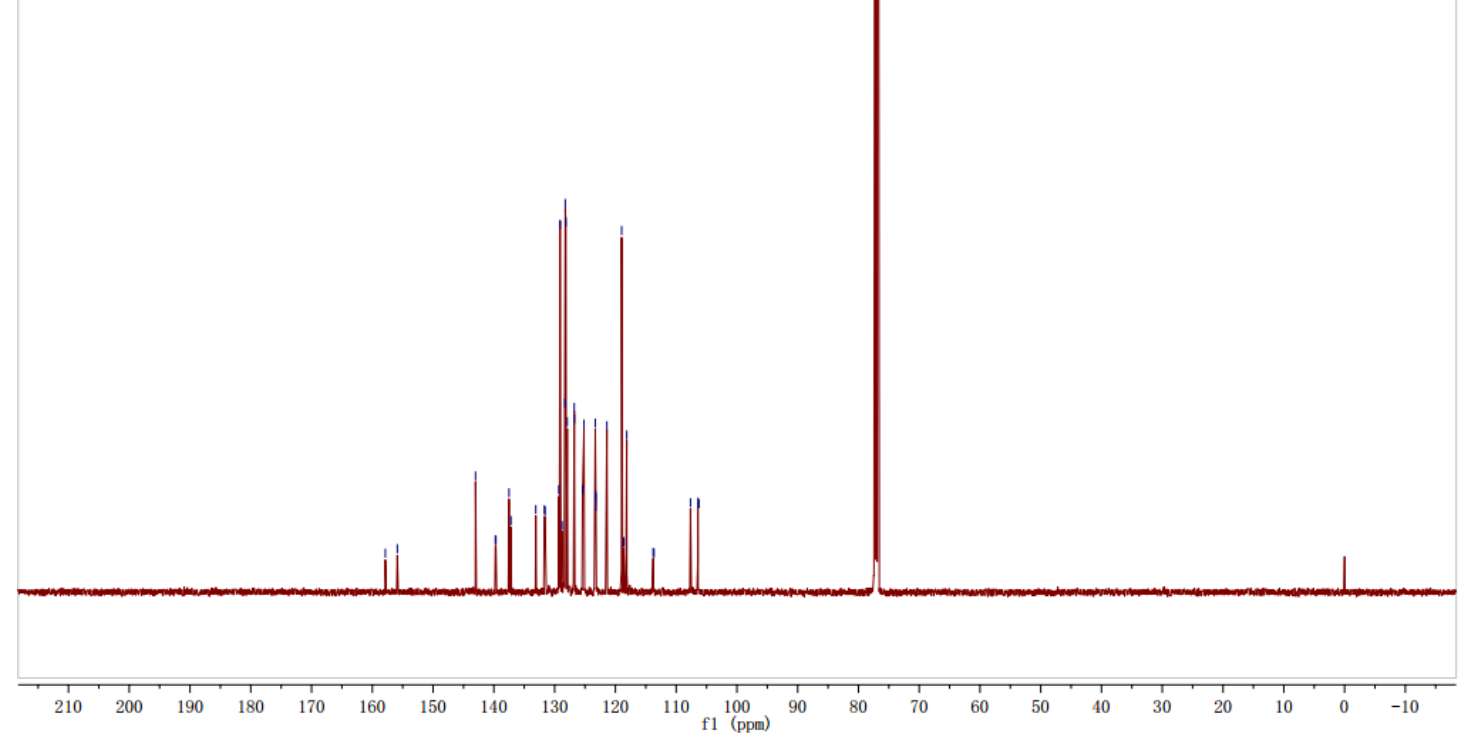



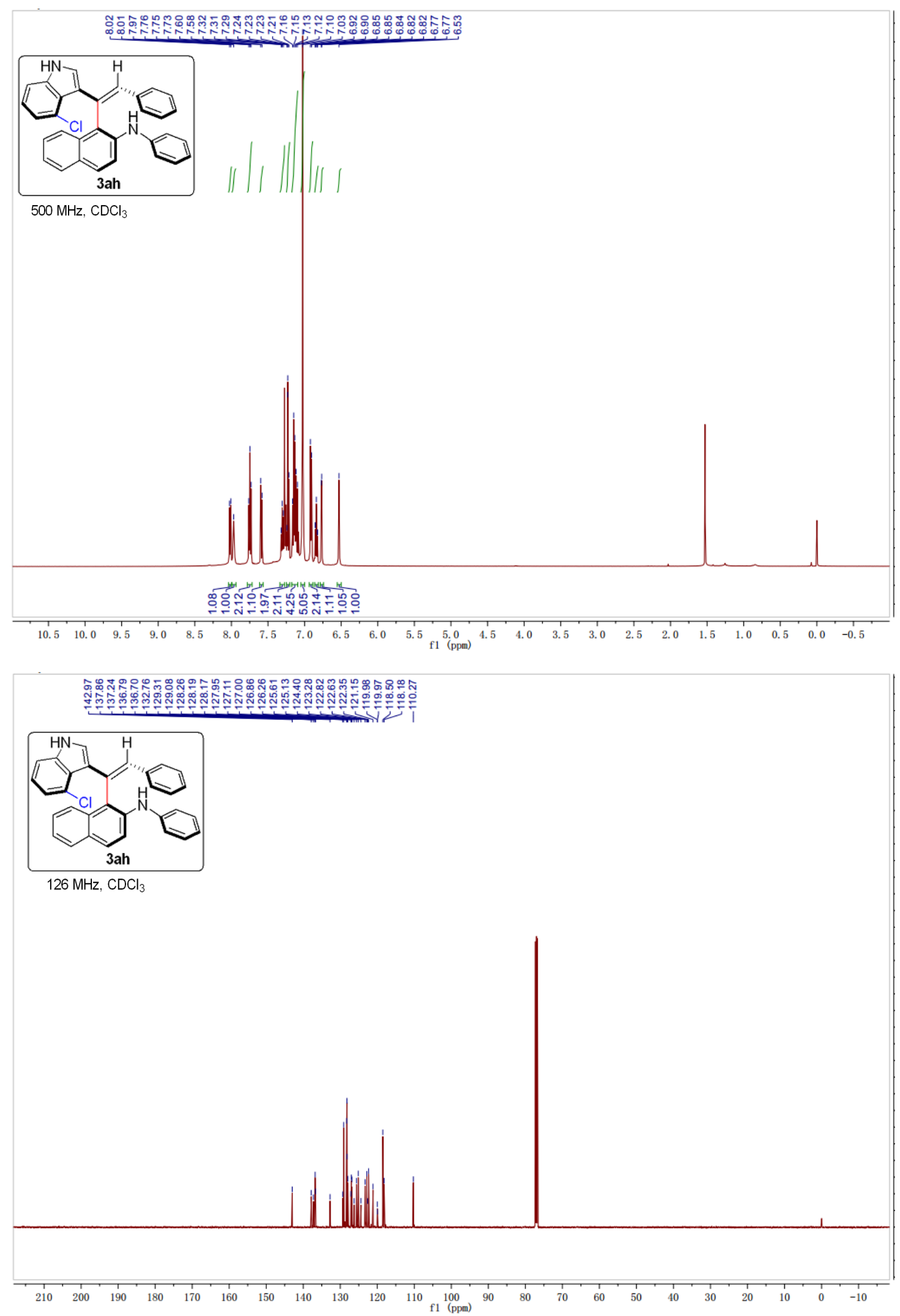


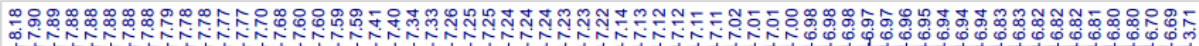

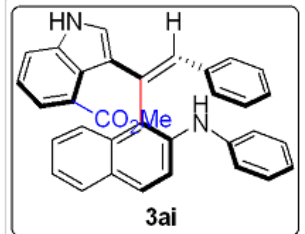

$500 \mathrm{MHz}, \mathrm{CDCl}_{3}$
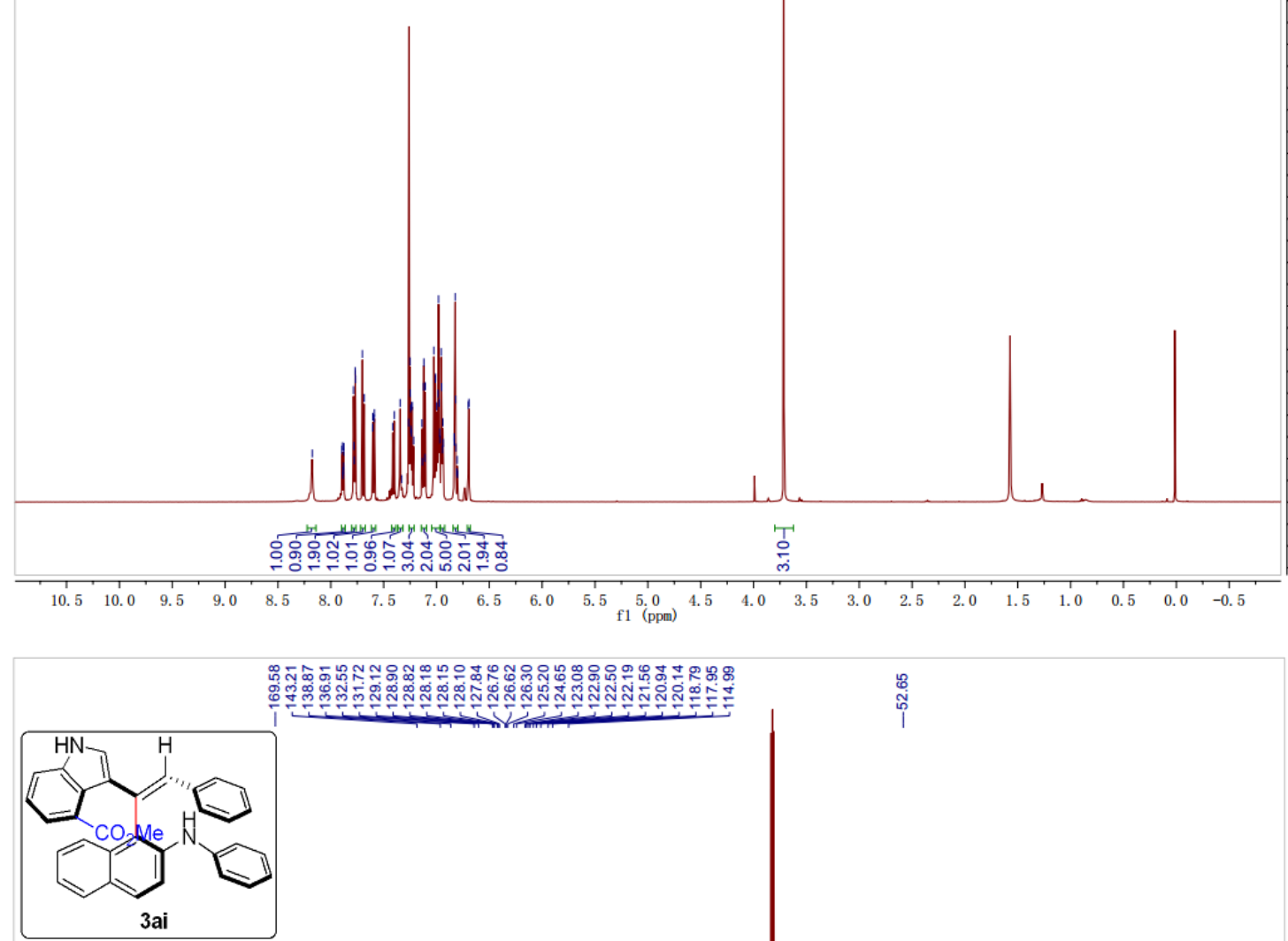

๓⿴囗十

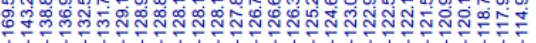

$126 \mathrm{MHz}, \mathrm{CDCl}_{3}$

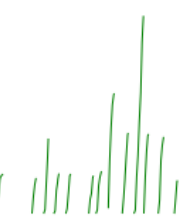

స్ำ
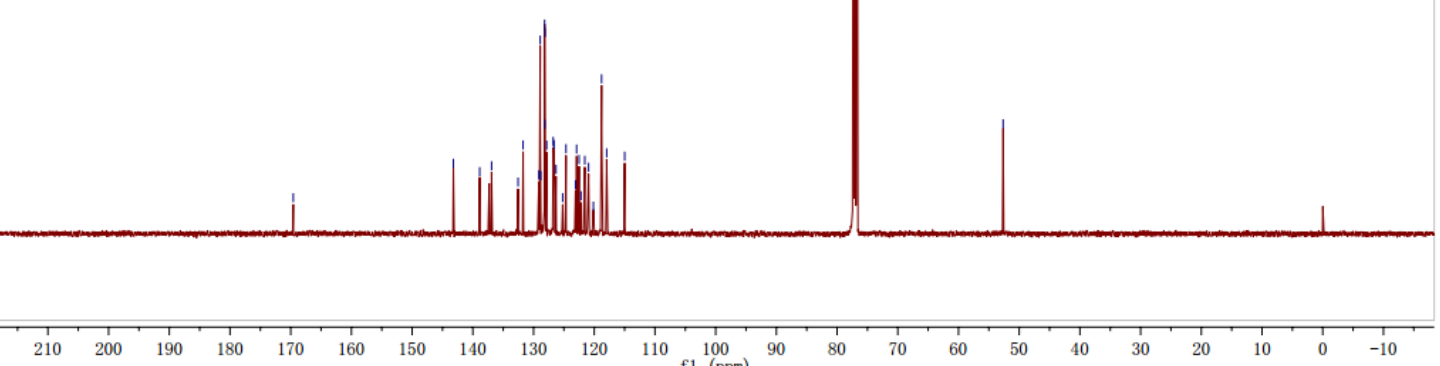

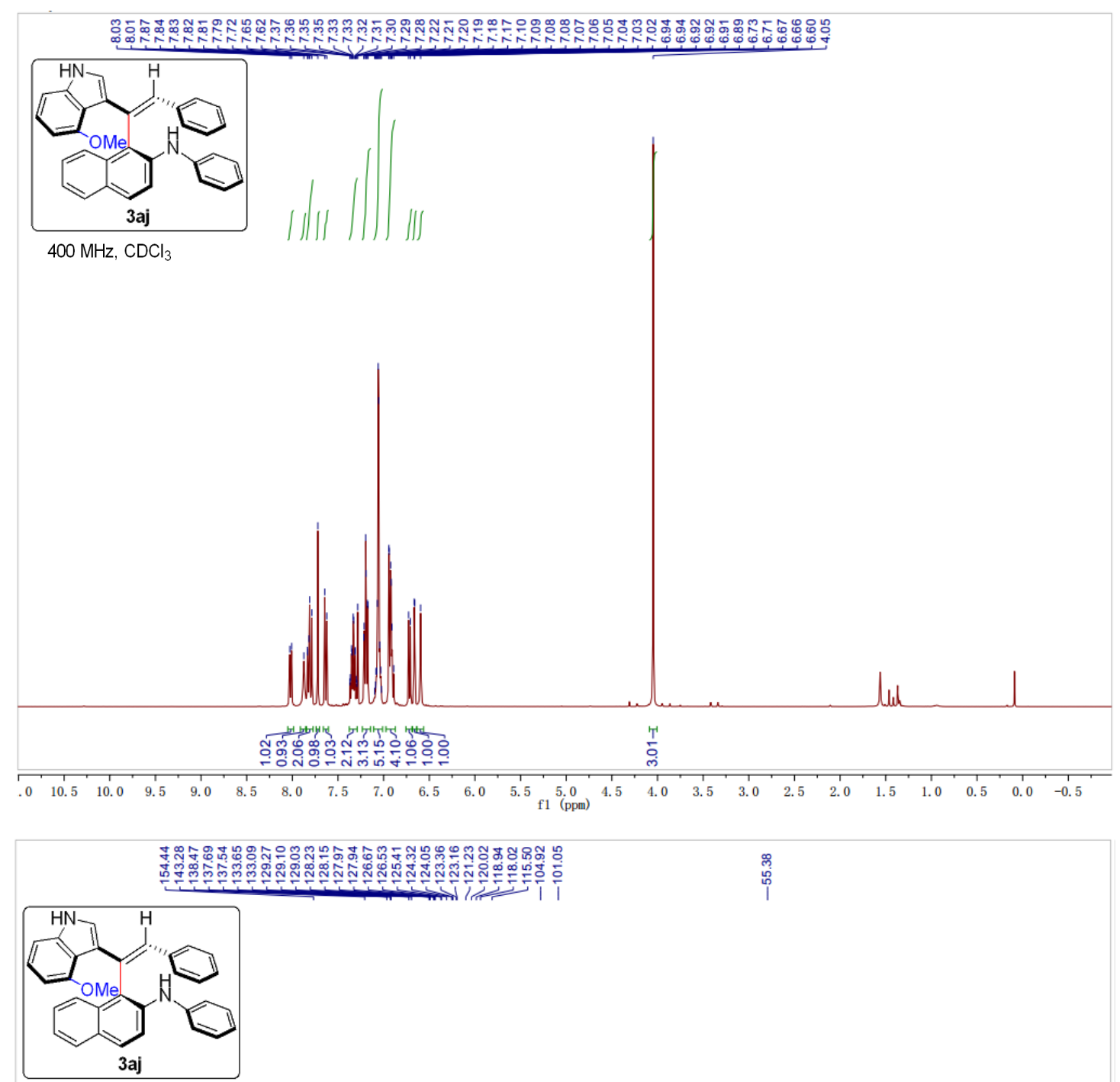

$101 \mathrm{MHz}, \mathrm{CDCl}_{3}$

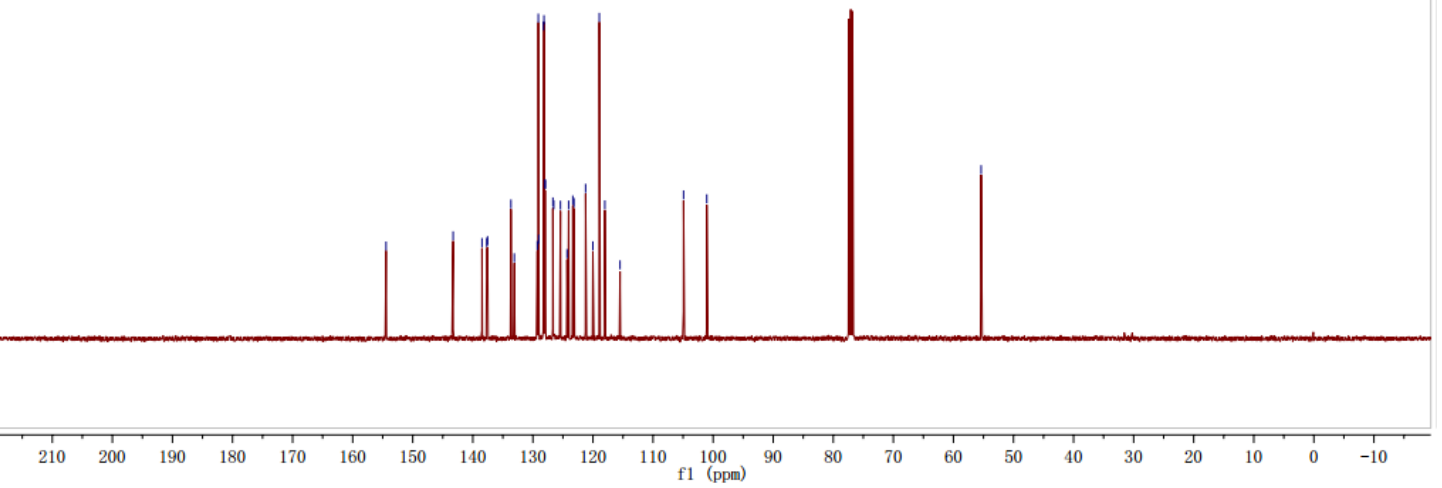


采盟

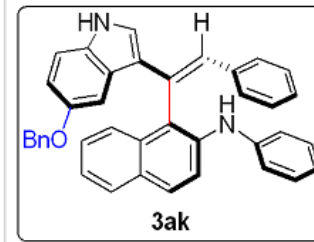

$600 \mathrm{MHz}, \mathrm{CDCl}_{3}$
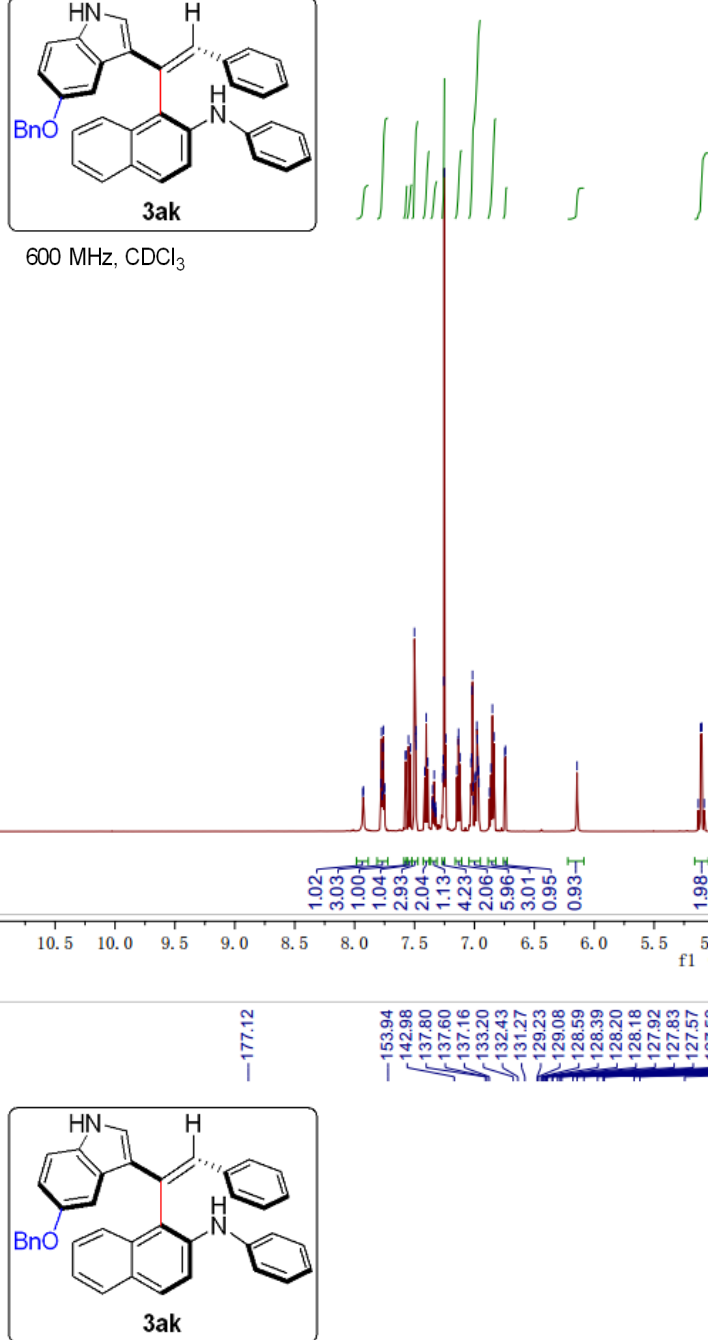

$151 \mathrm{MHz}, \mathrm{CDCl}_{3}$

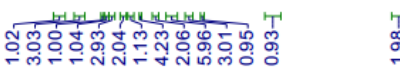

\section{悉}

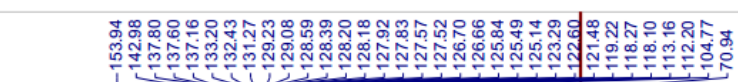

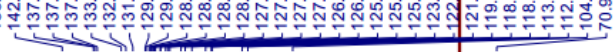

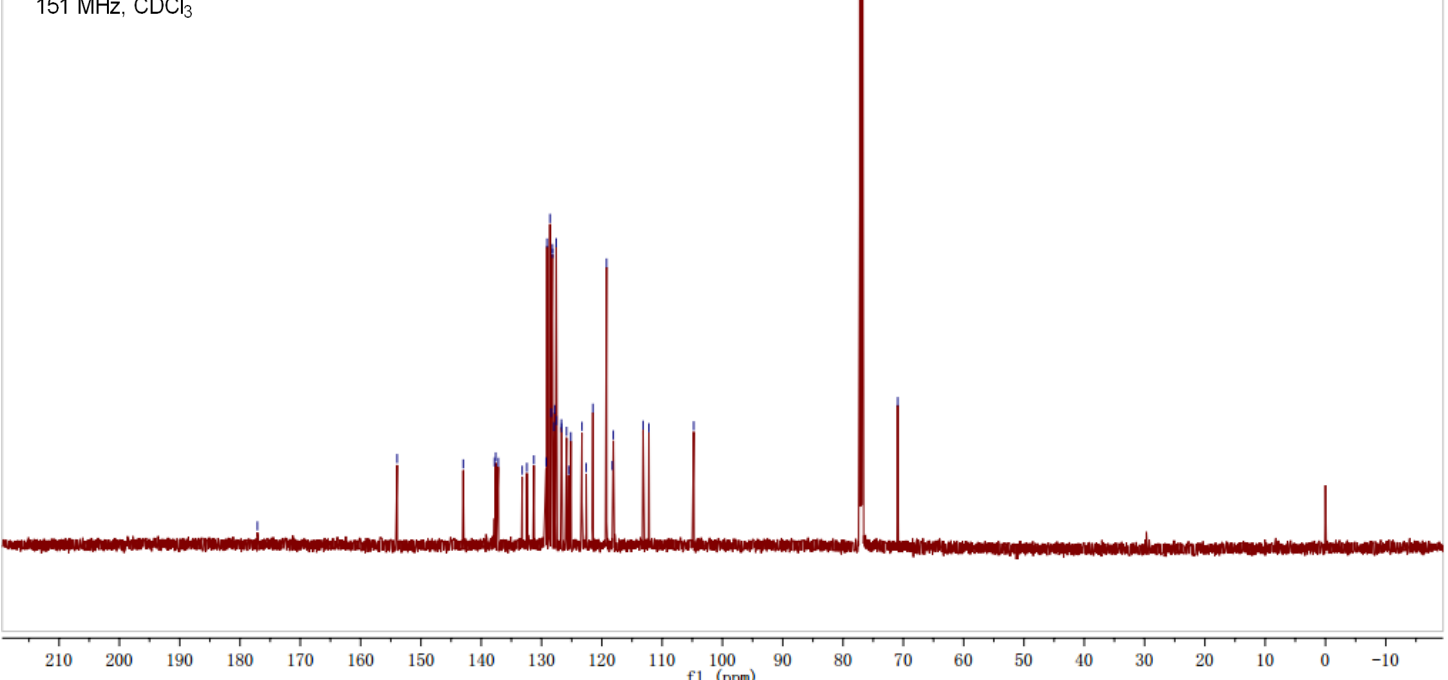



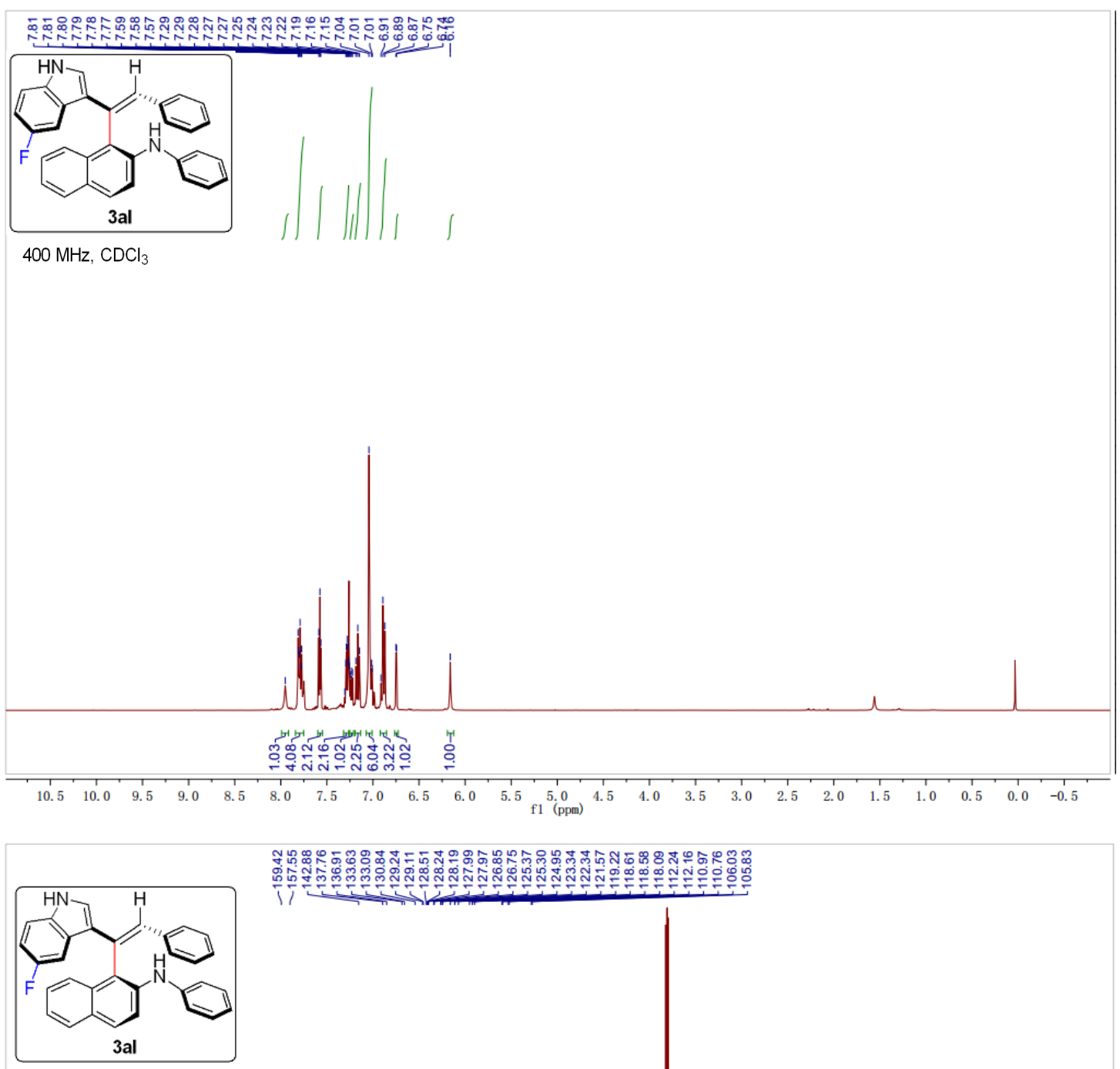

$126 \mathrm{MHz}, \mathrm{CDCl}_{3}$

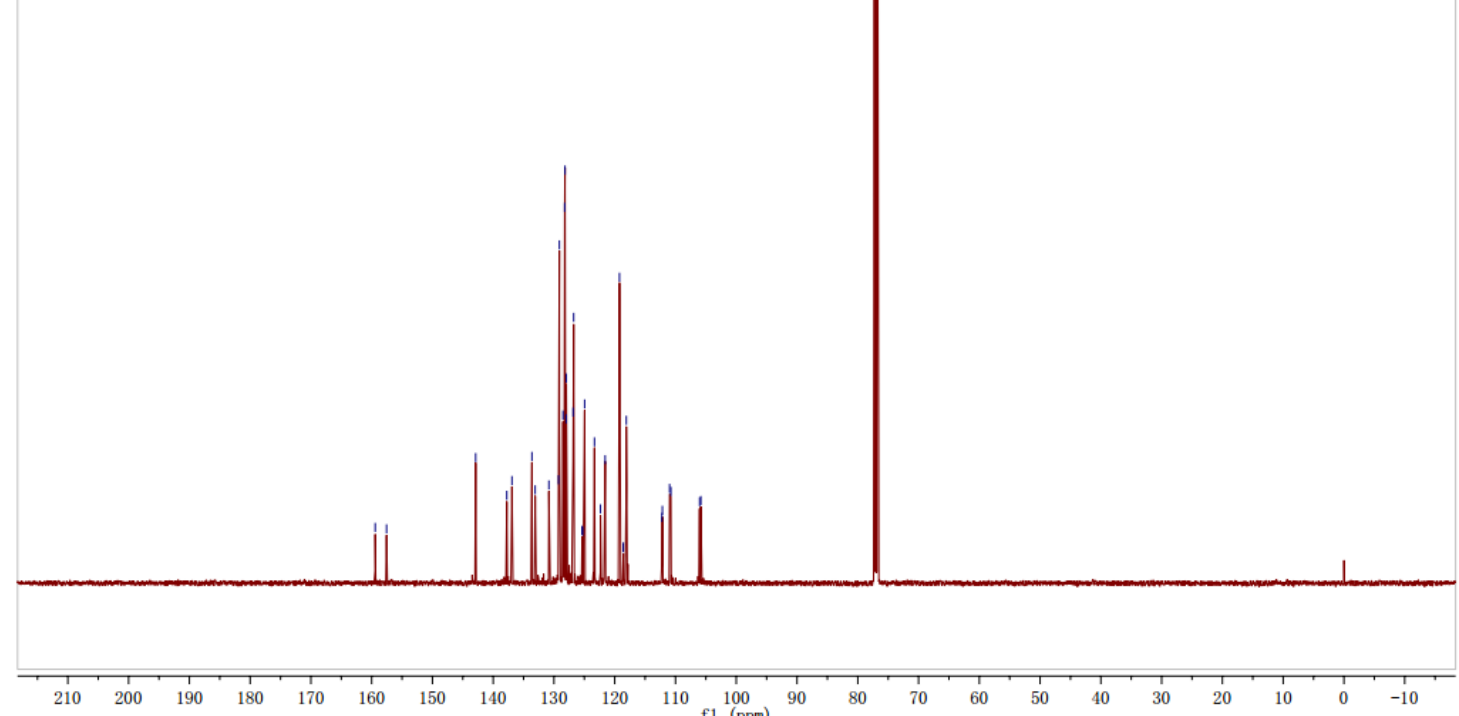




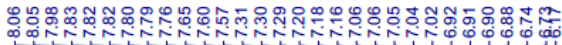
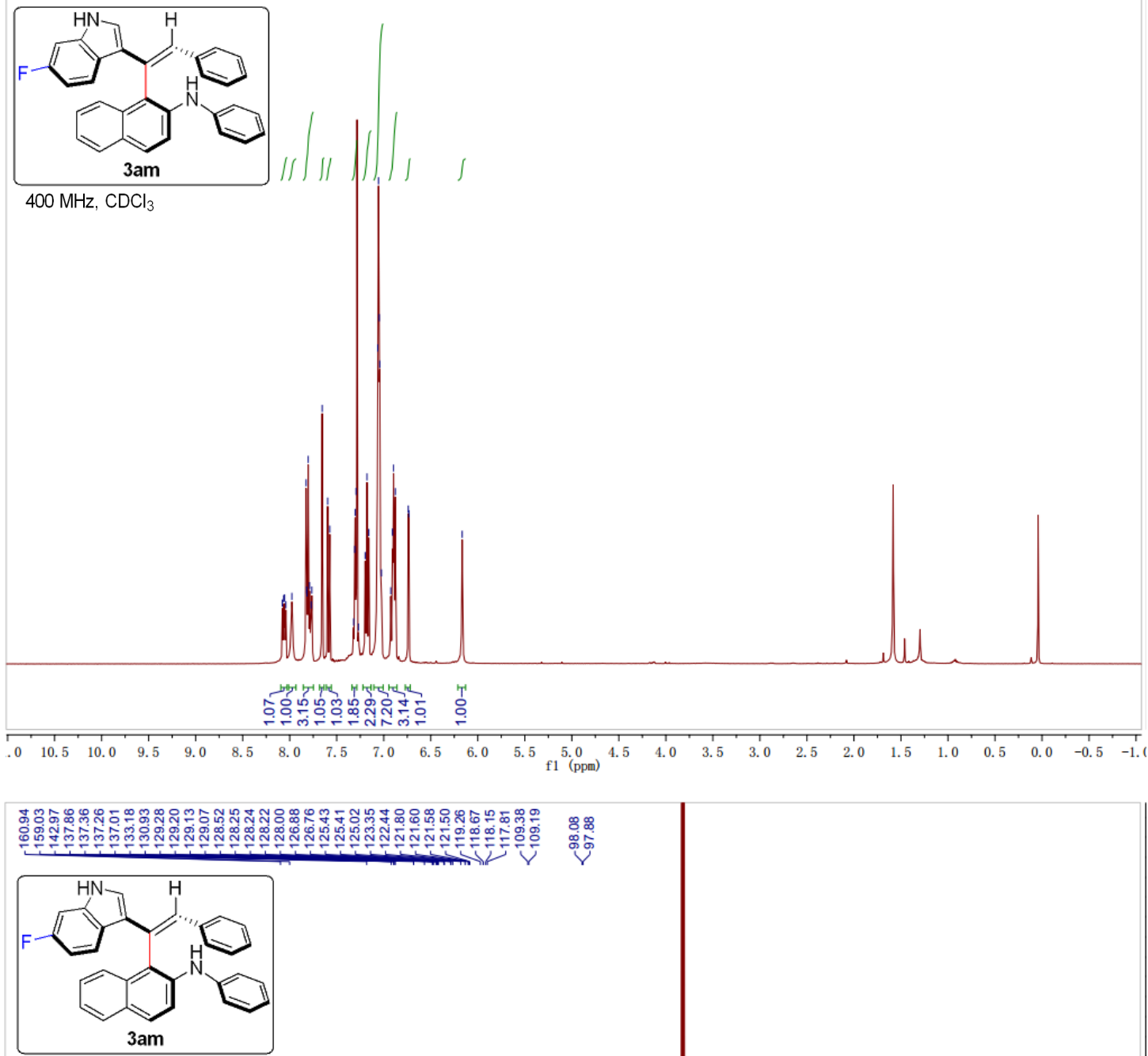

$126 \mathrm{MHz}, \mathrm{CDCl}_{3}$
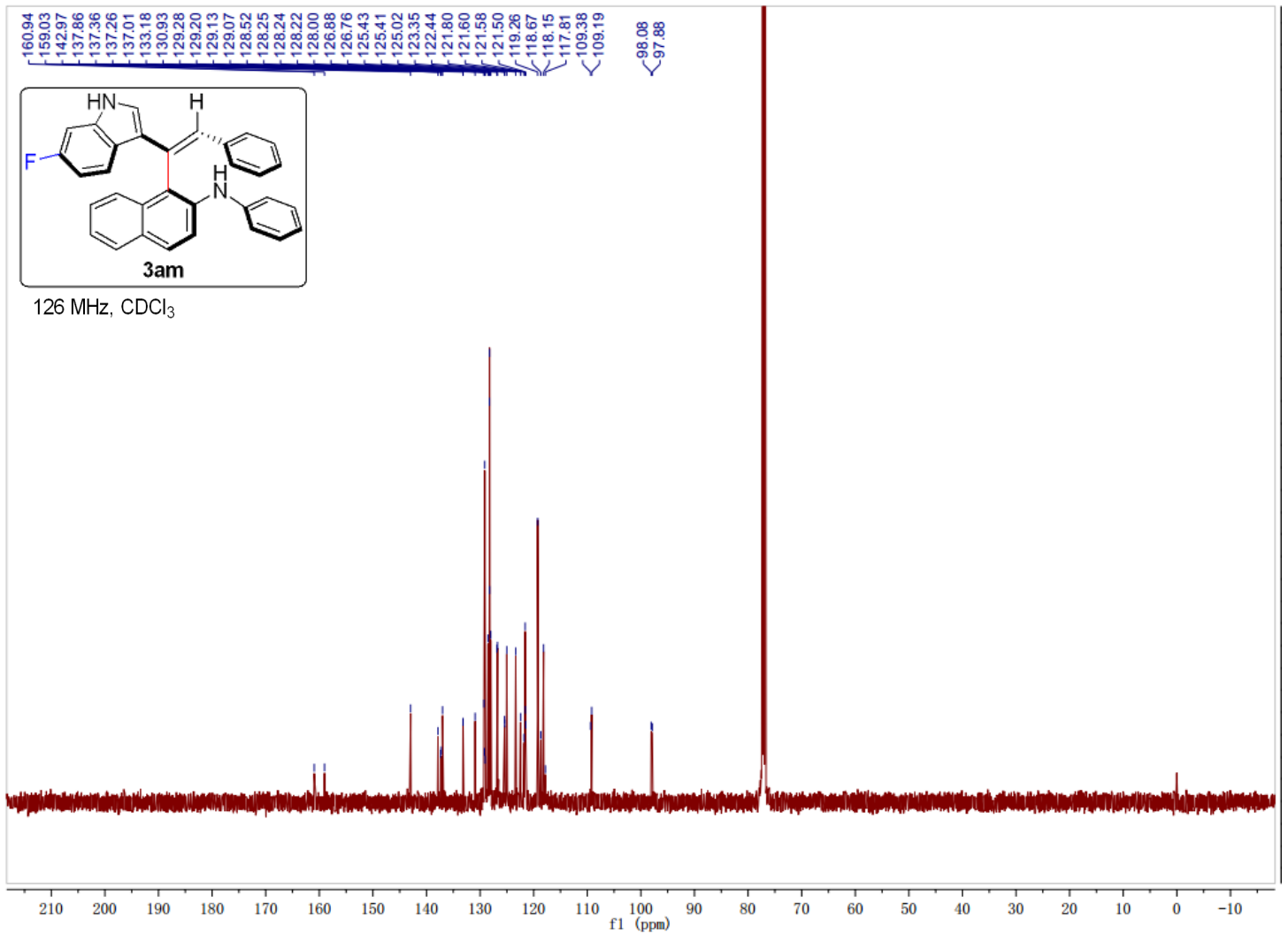

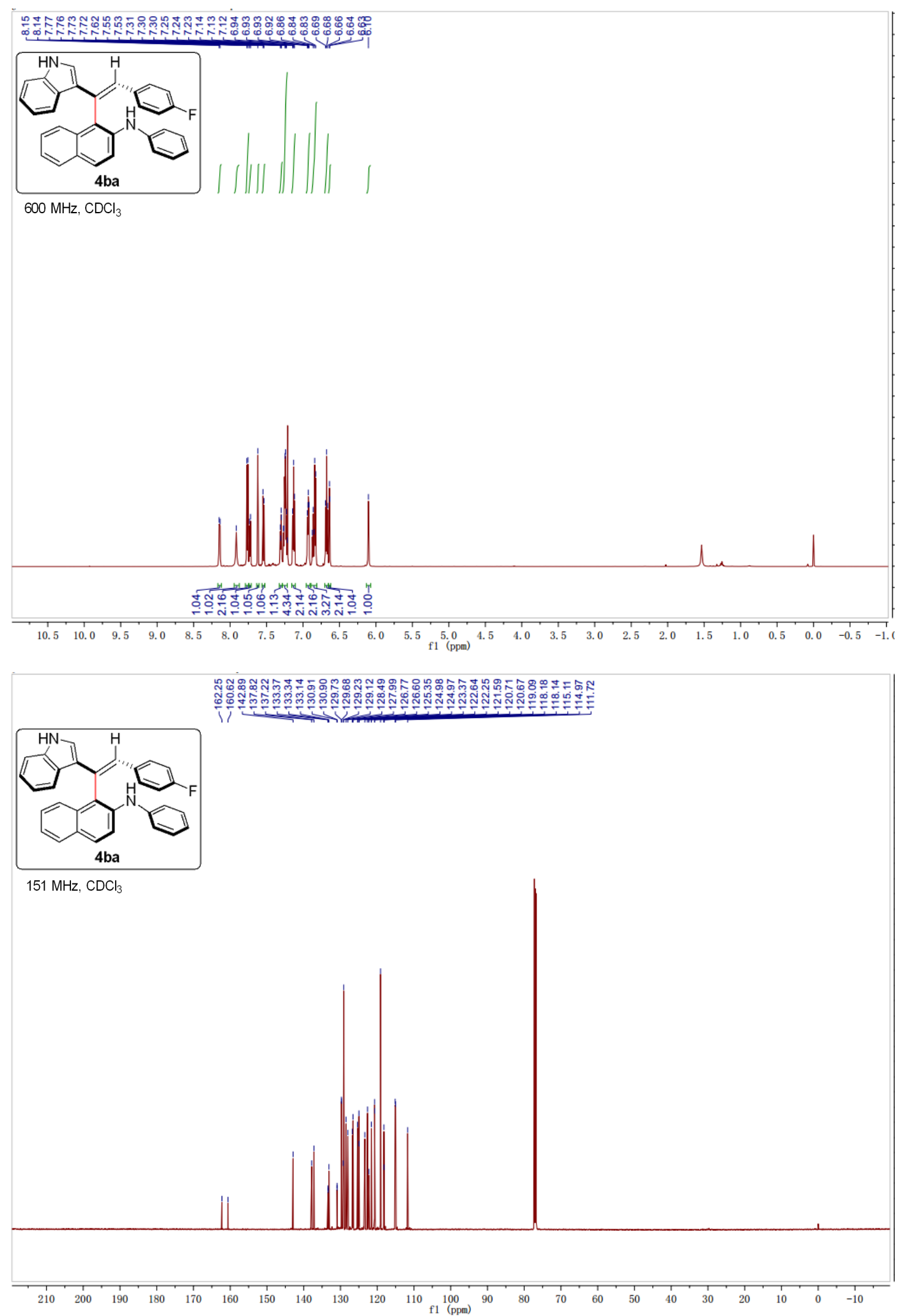

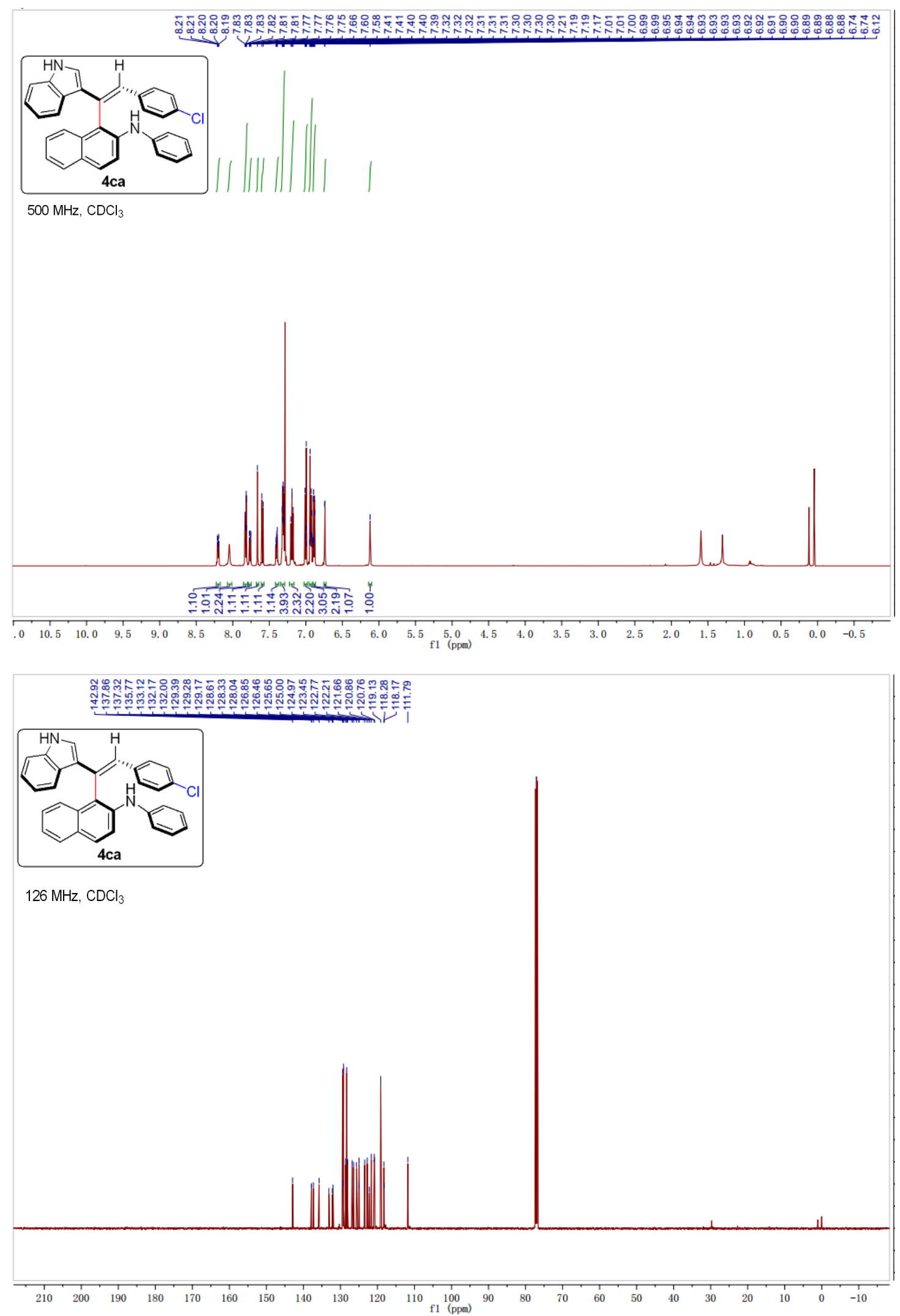

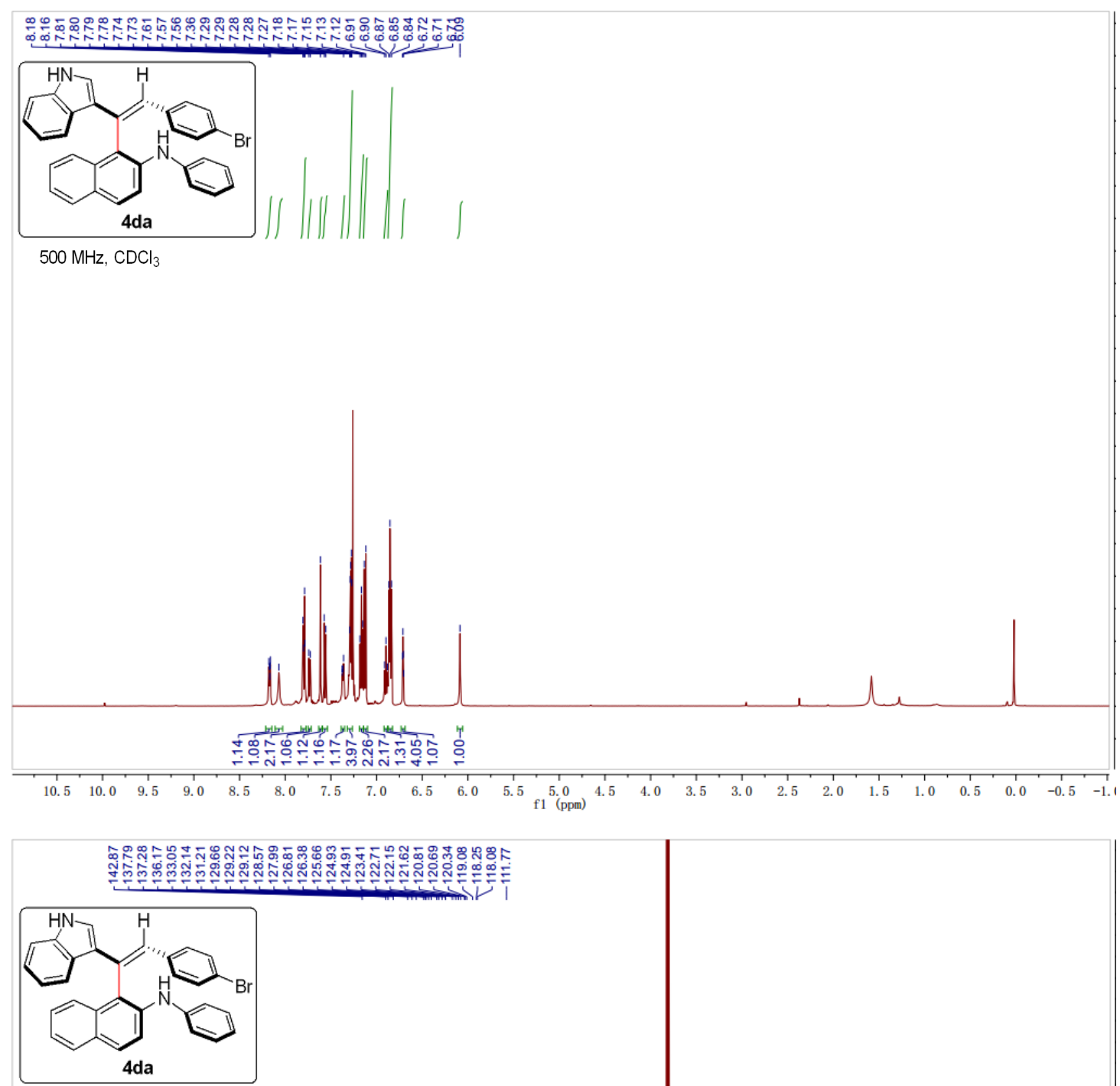

$126 \mathrm{MHz}, \mathrm{CDCl}_{3}$

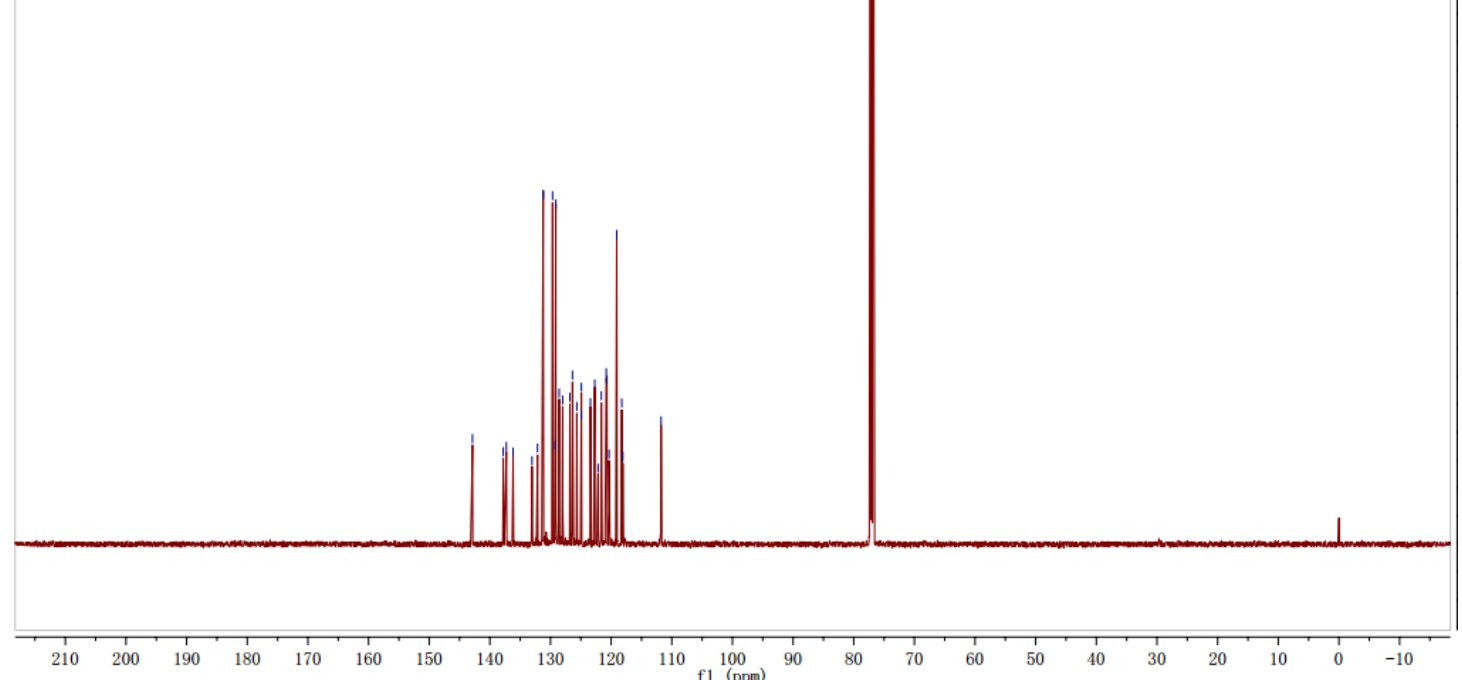



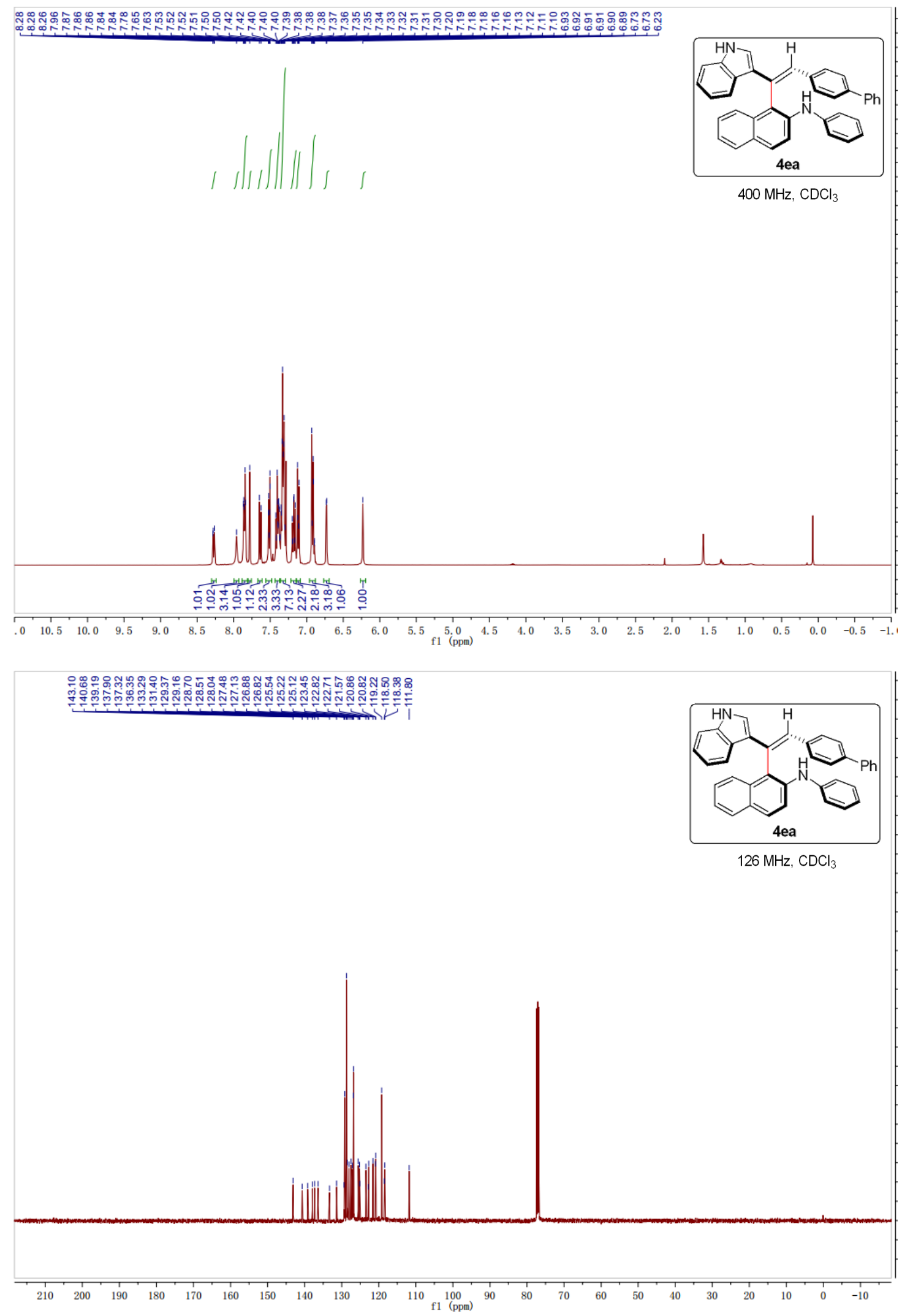


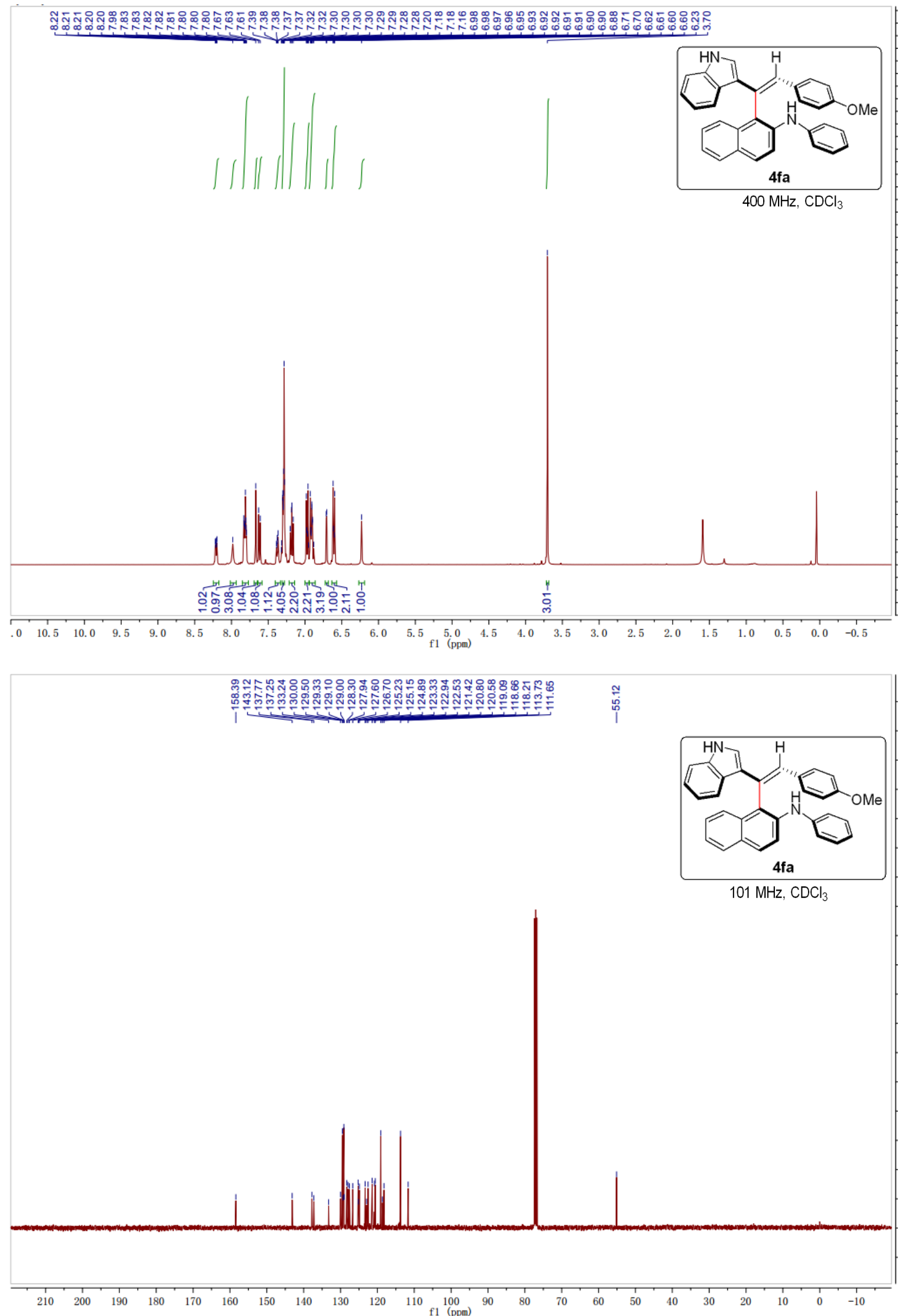




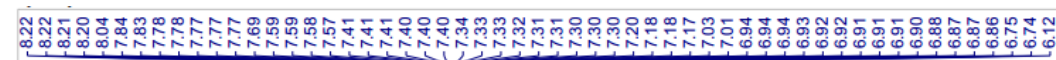
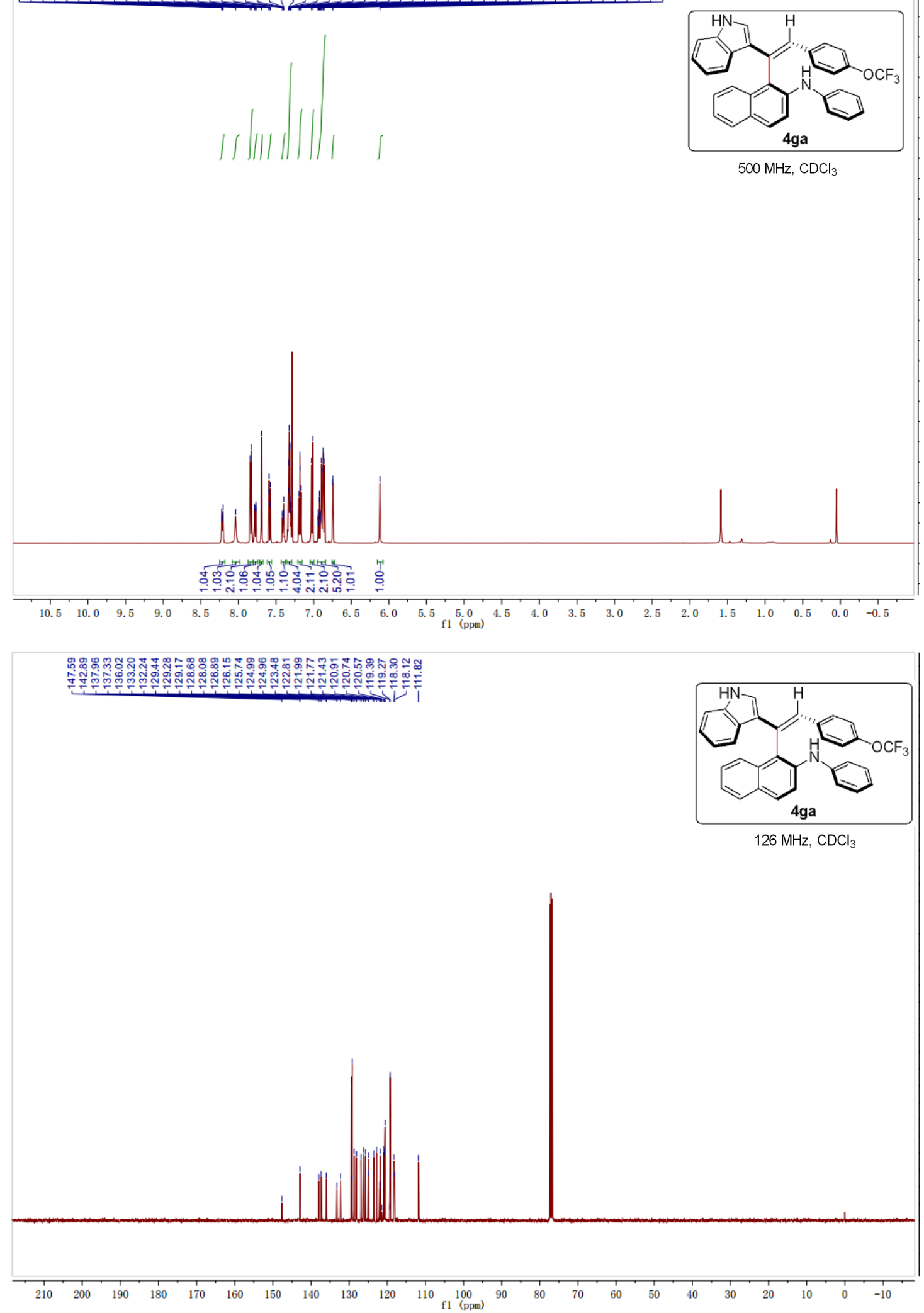


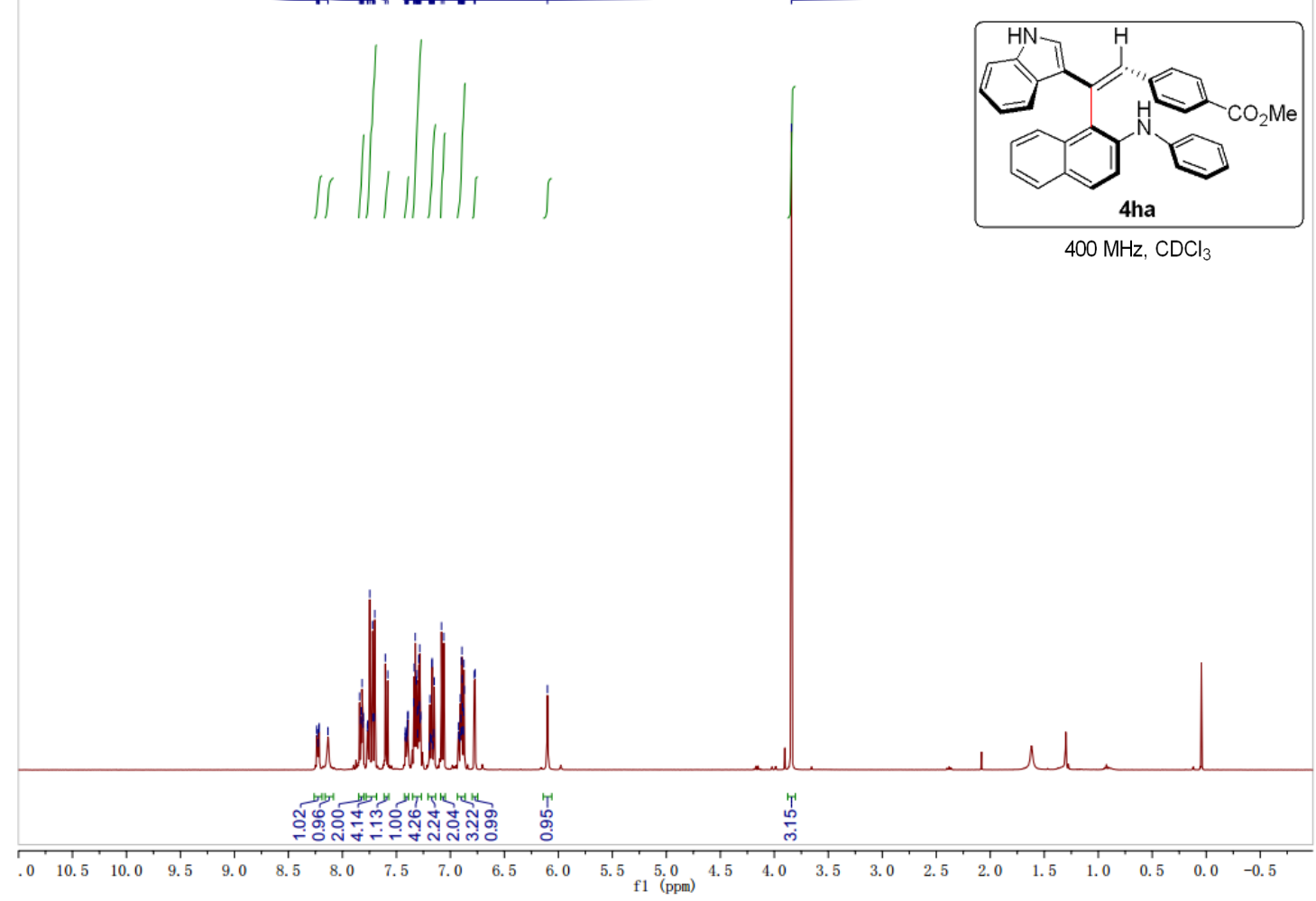

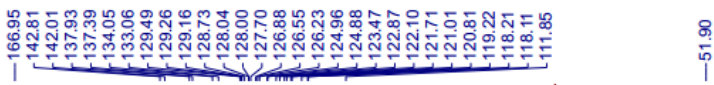

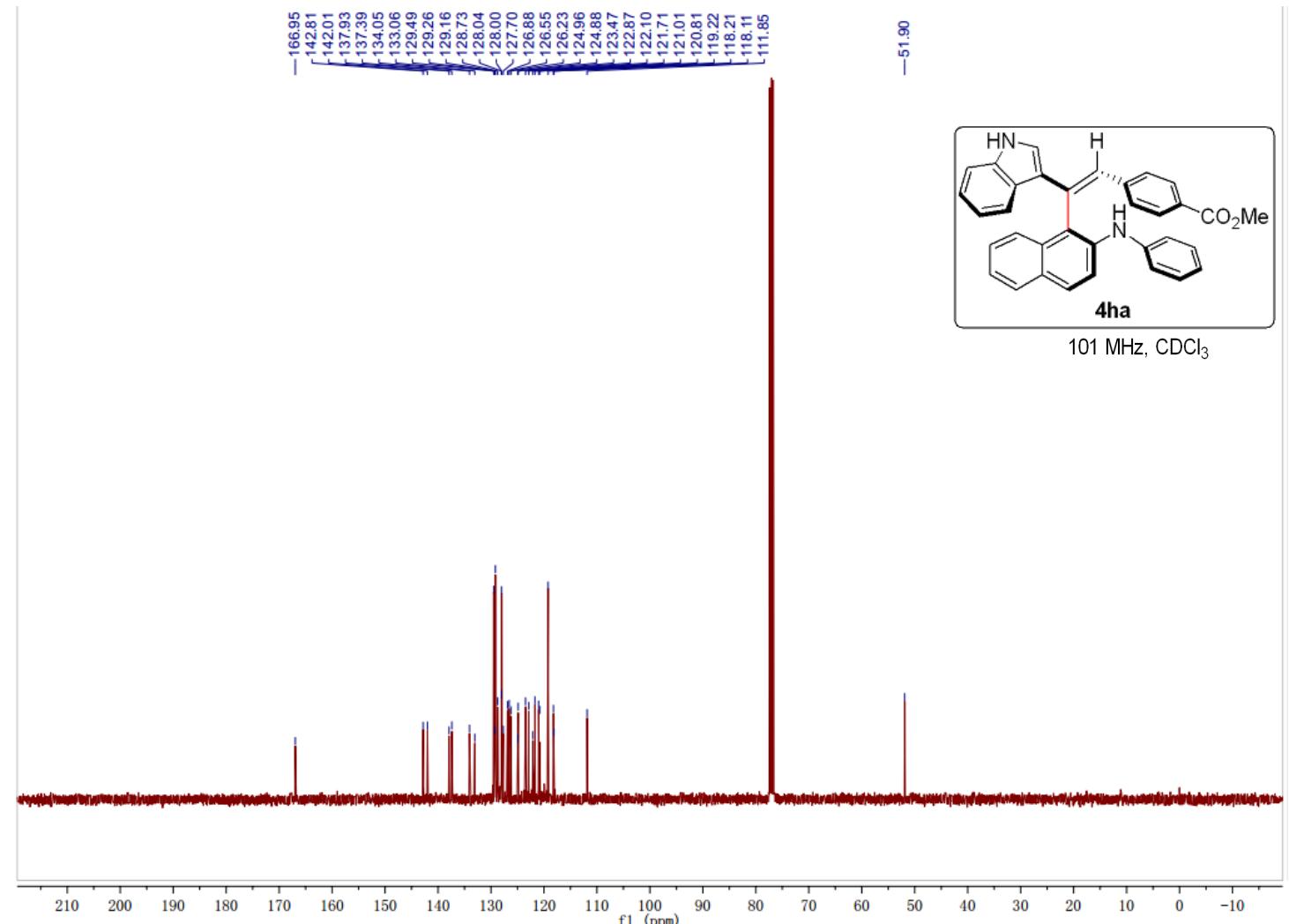



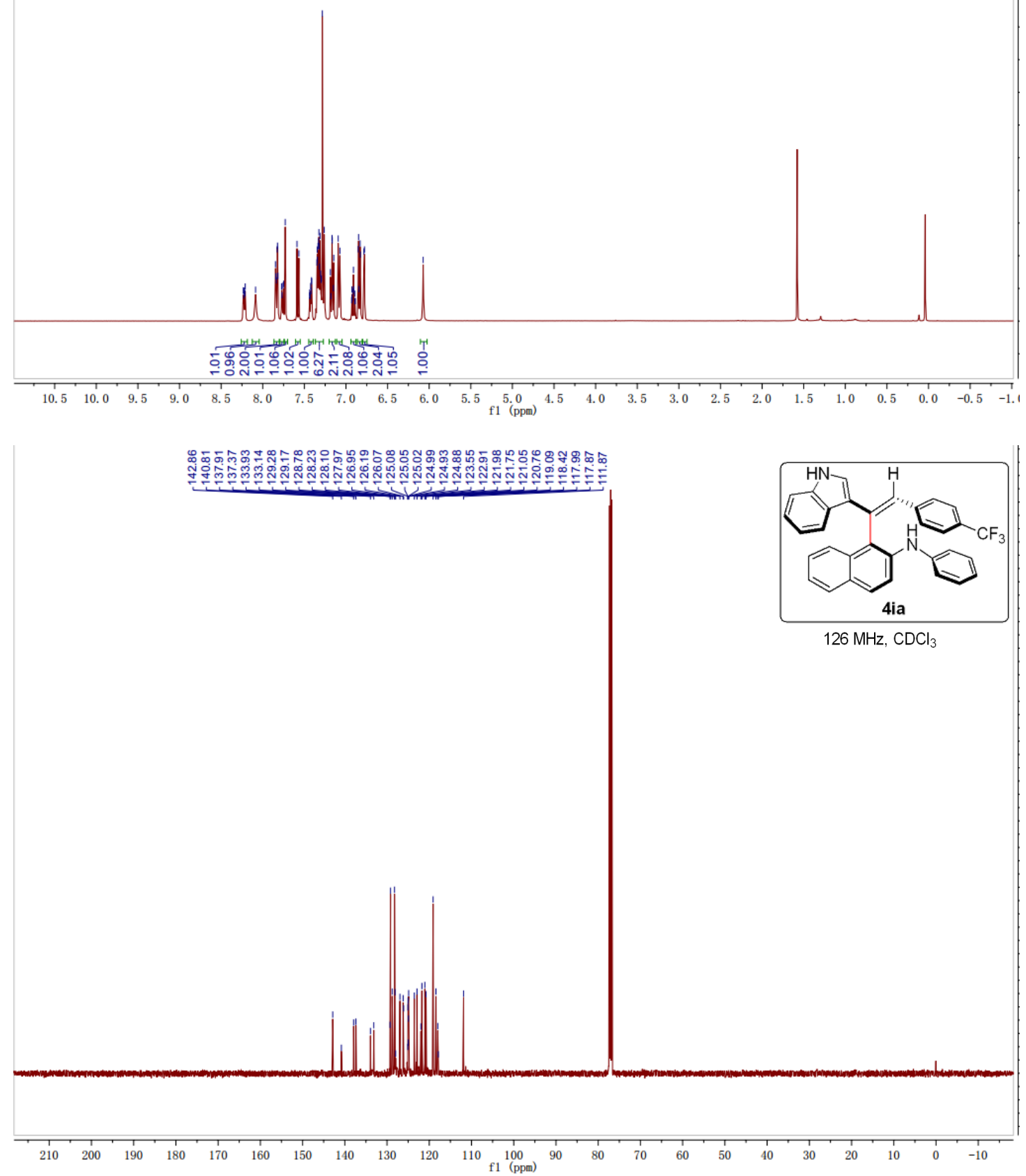

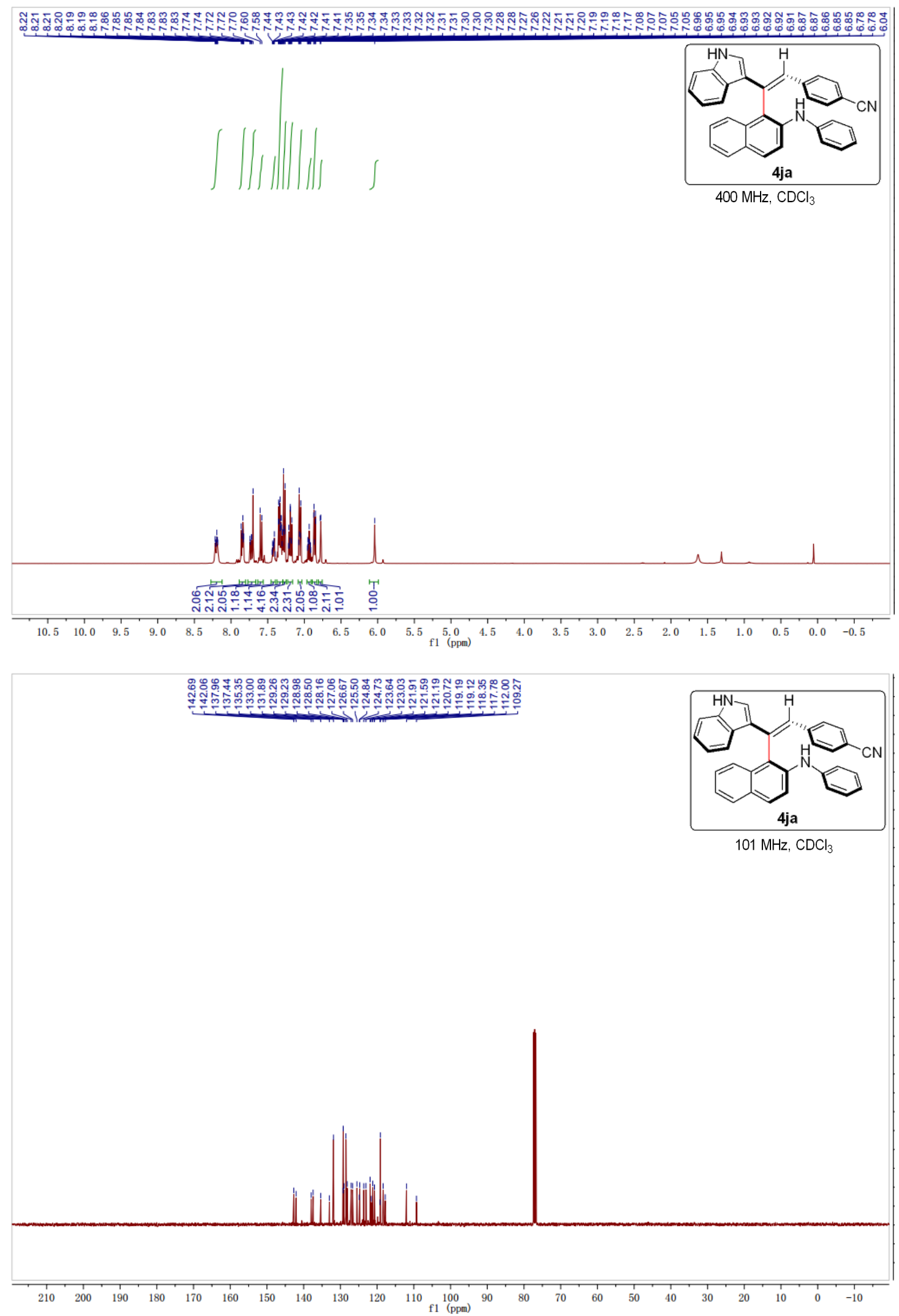


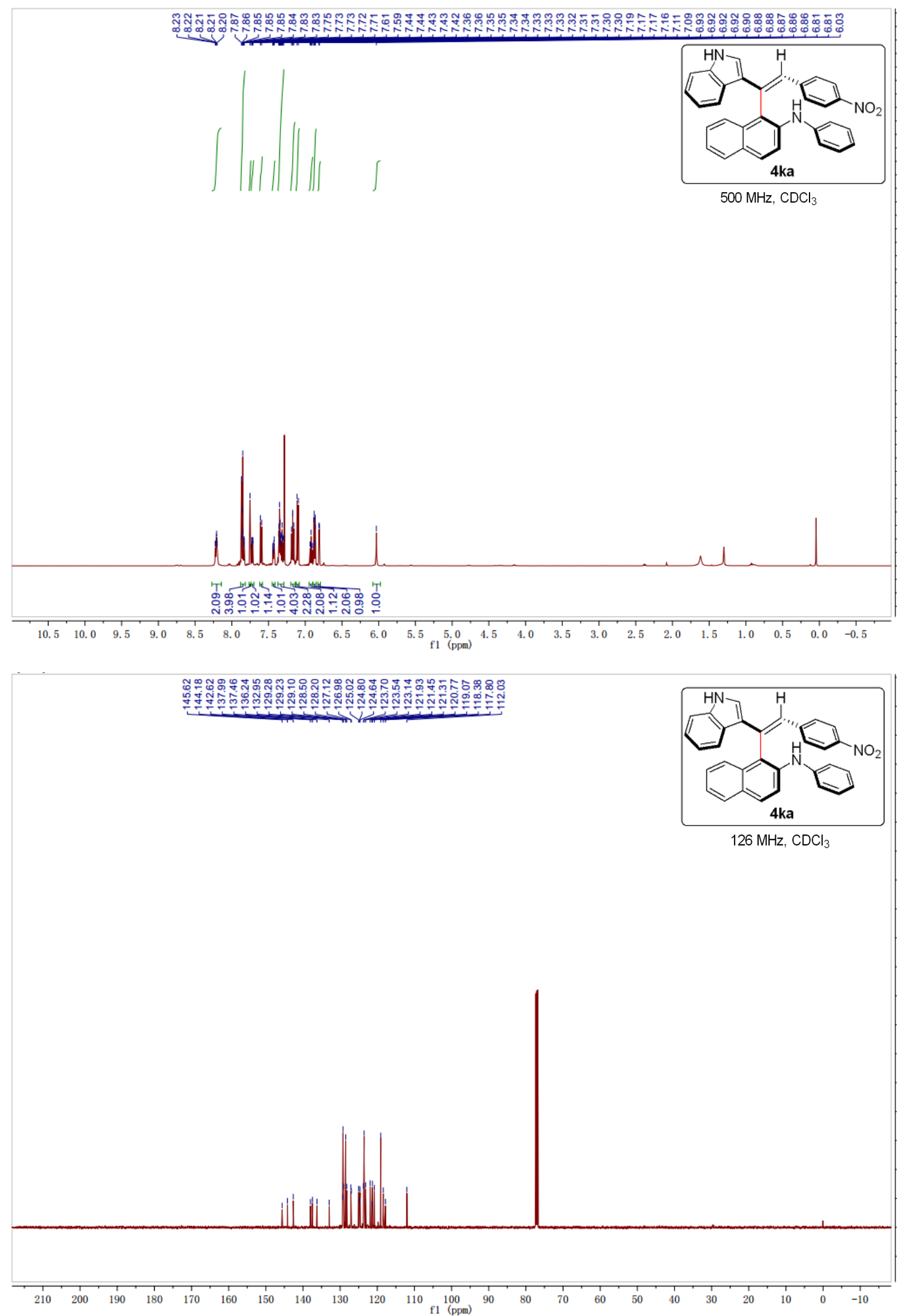




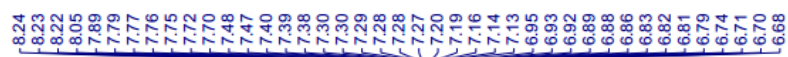

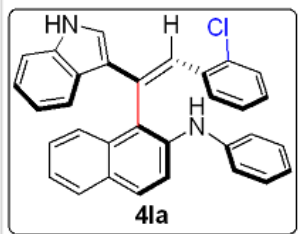

$500 \mathrm{MHz}, \mathrm{CDCl}_{3}$
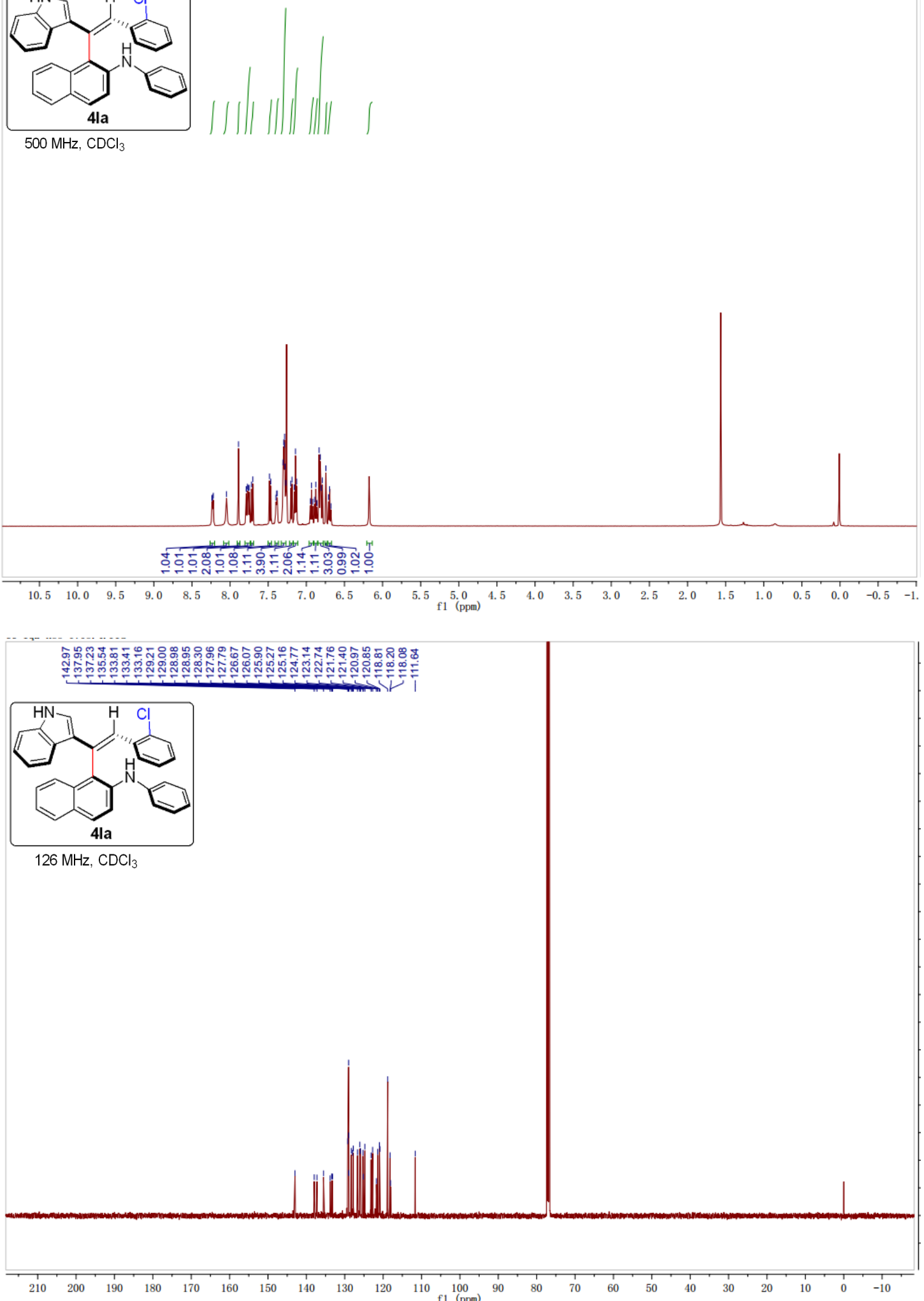

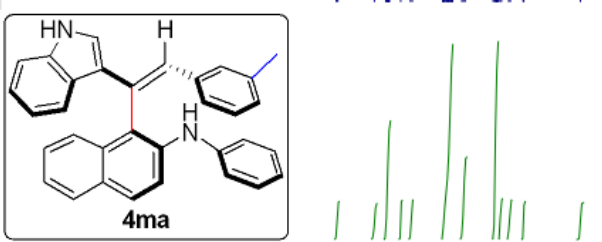

$600 \mathrm{MHz}, \mathrm{CDCl}_{3}$

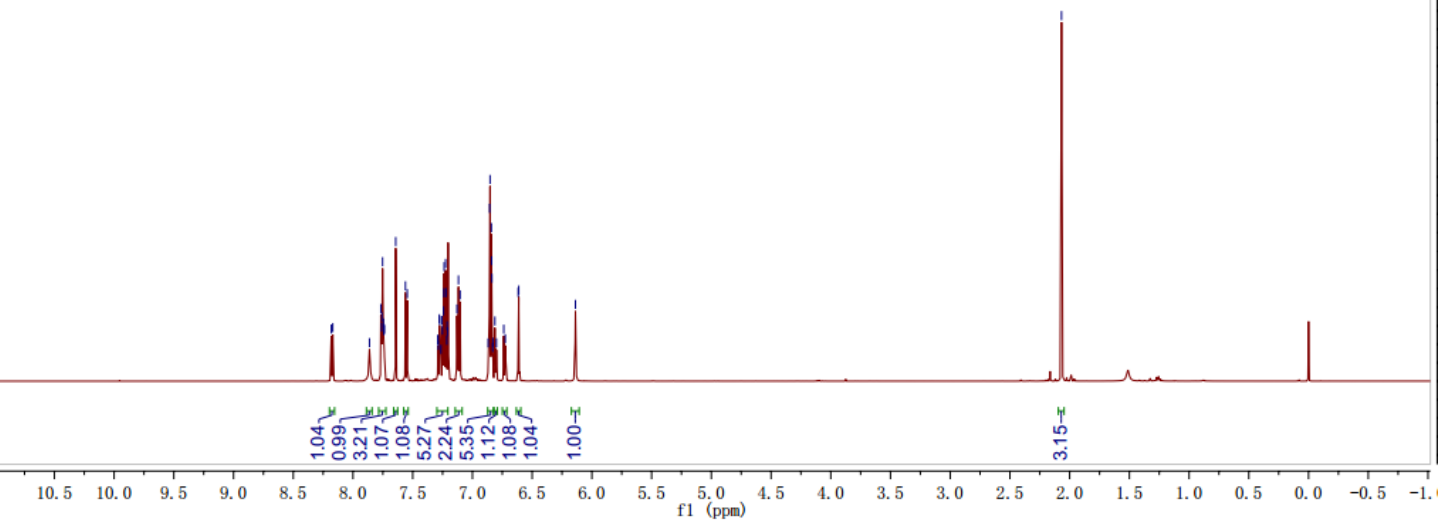

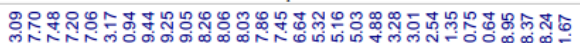

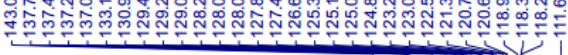

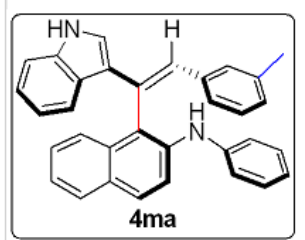

$151 \mathrm{MHz}, \mathrm{CDCl}$

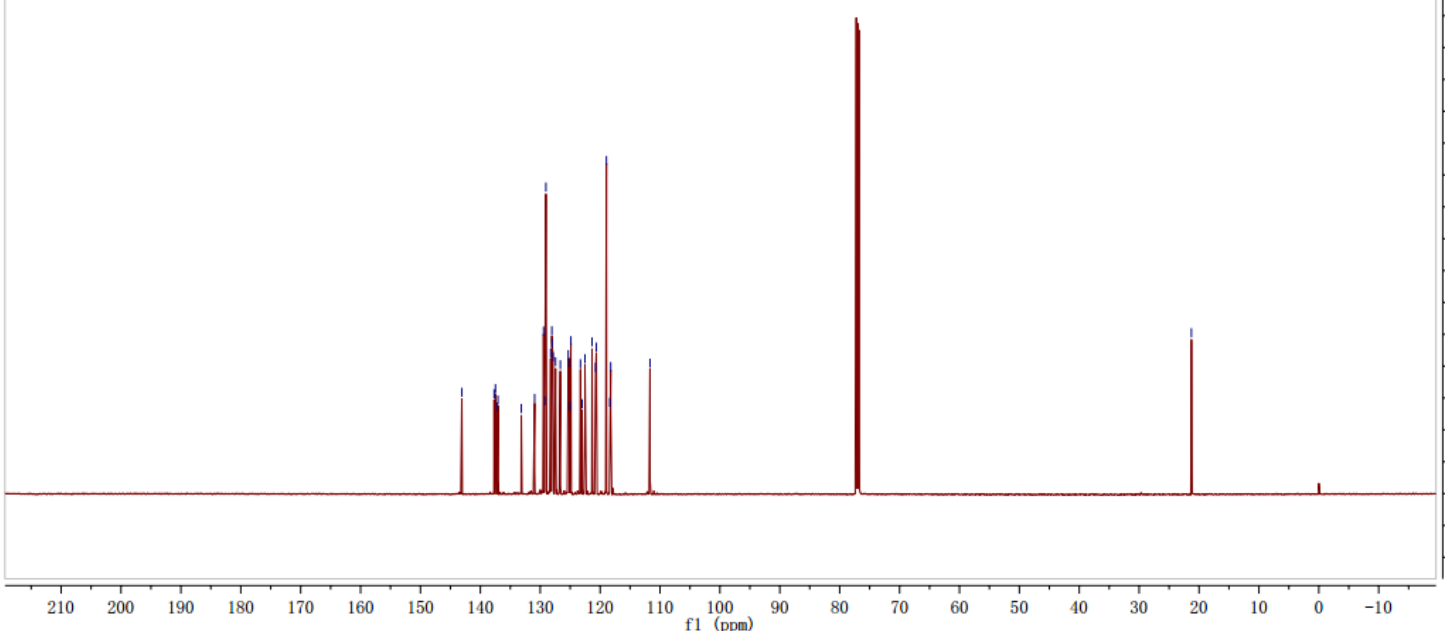




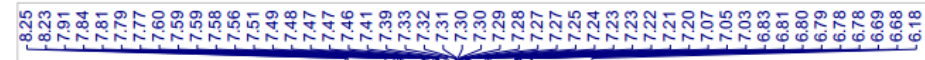
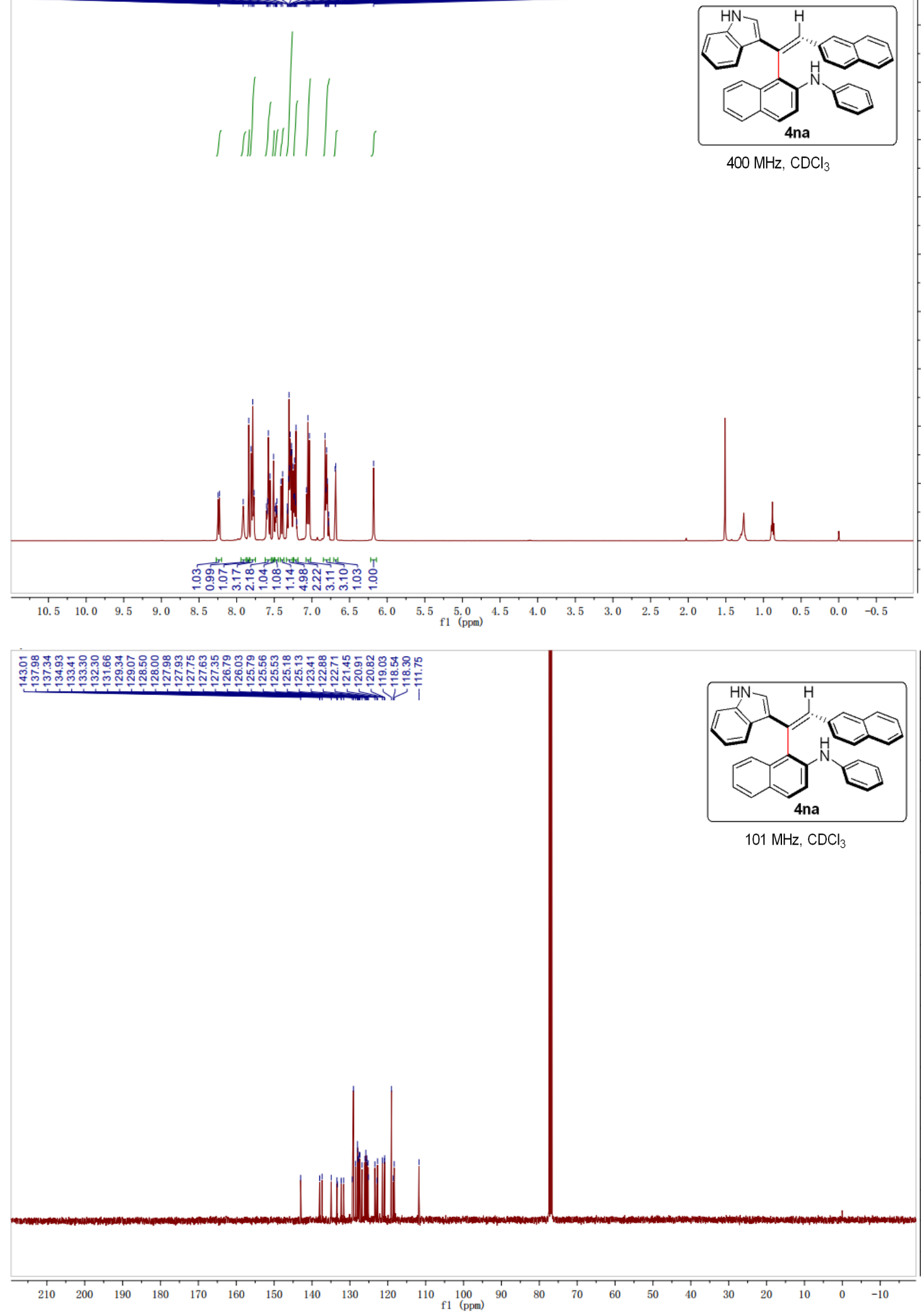


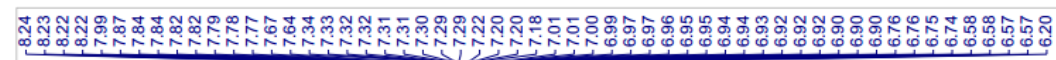

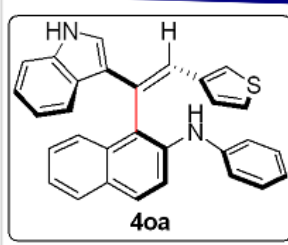

$400 \mathrm{MHz}, \mathrm{CDCl}_{3}$
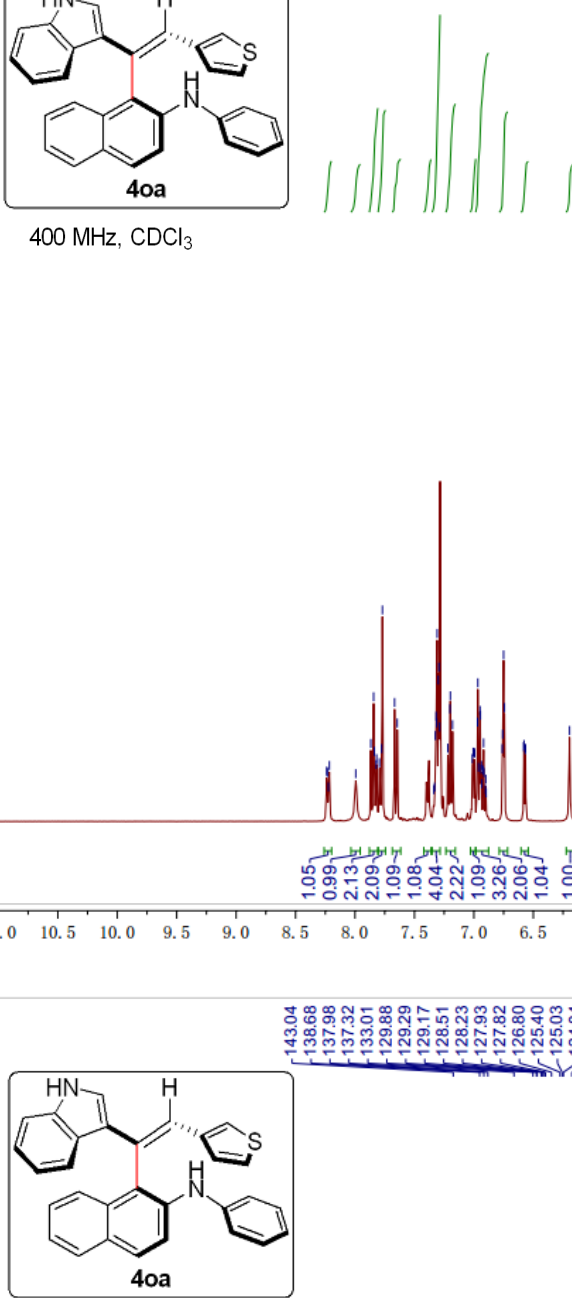

$126 \mathrm{MHz}, \mathrm{CDCl}_{3}$

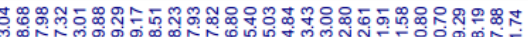

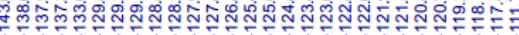

Hithy

政

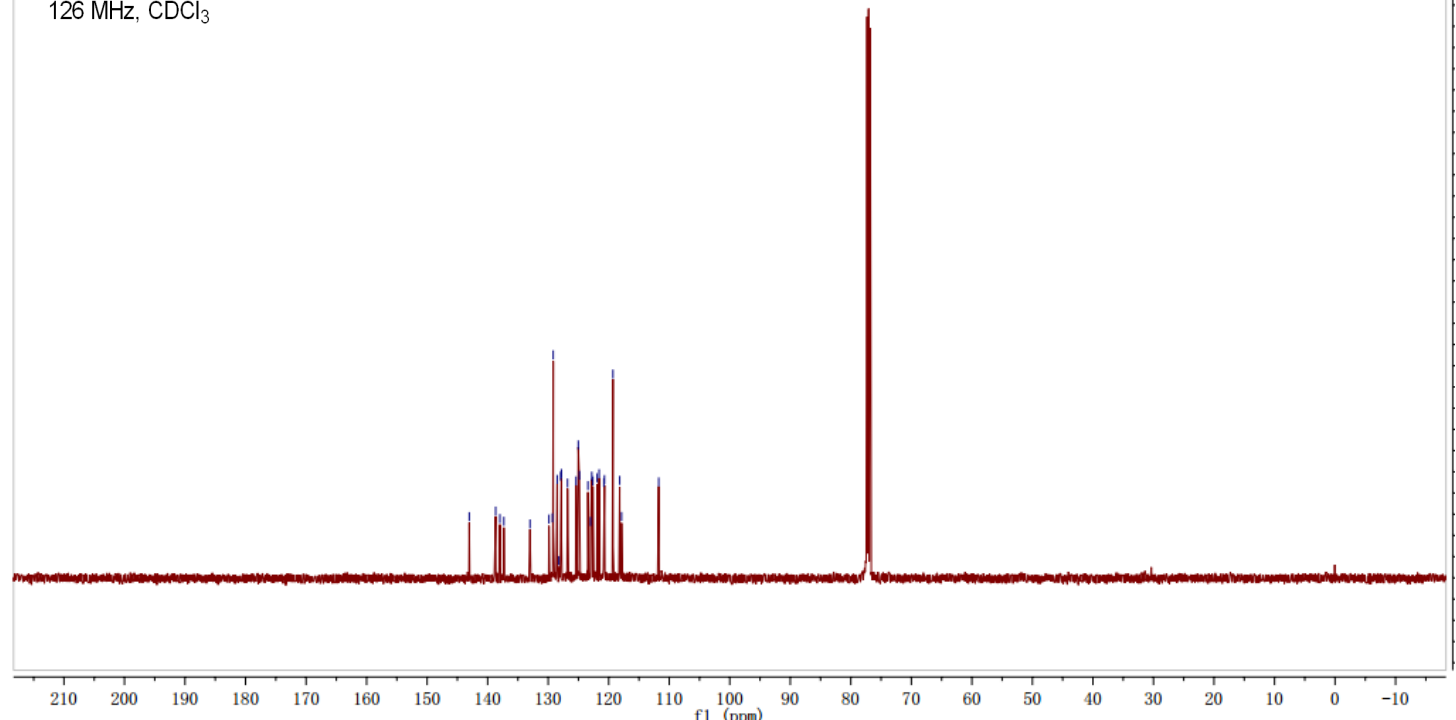



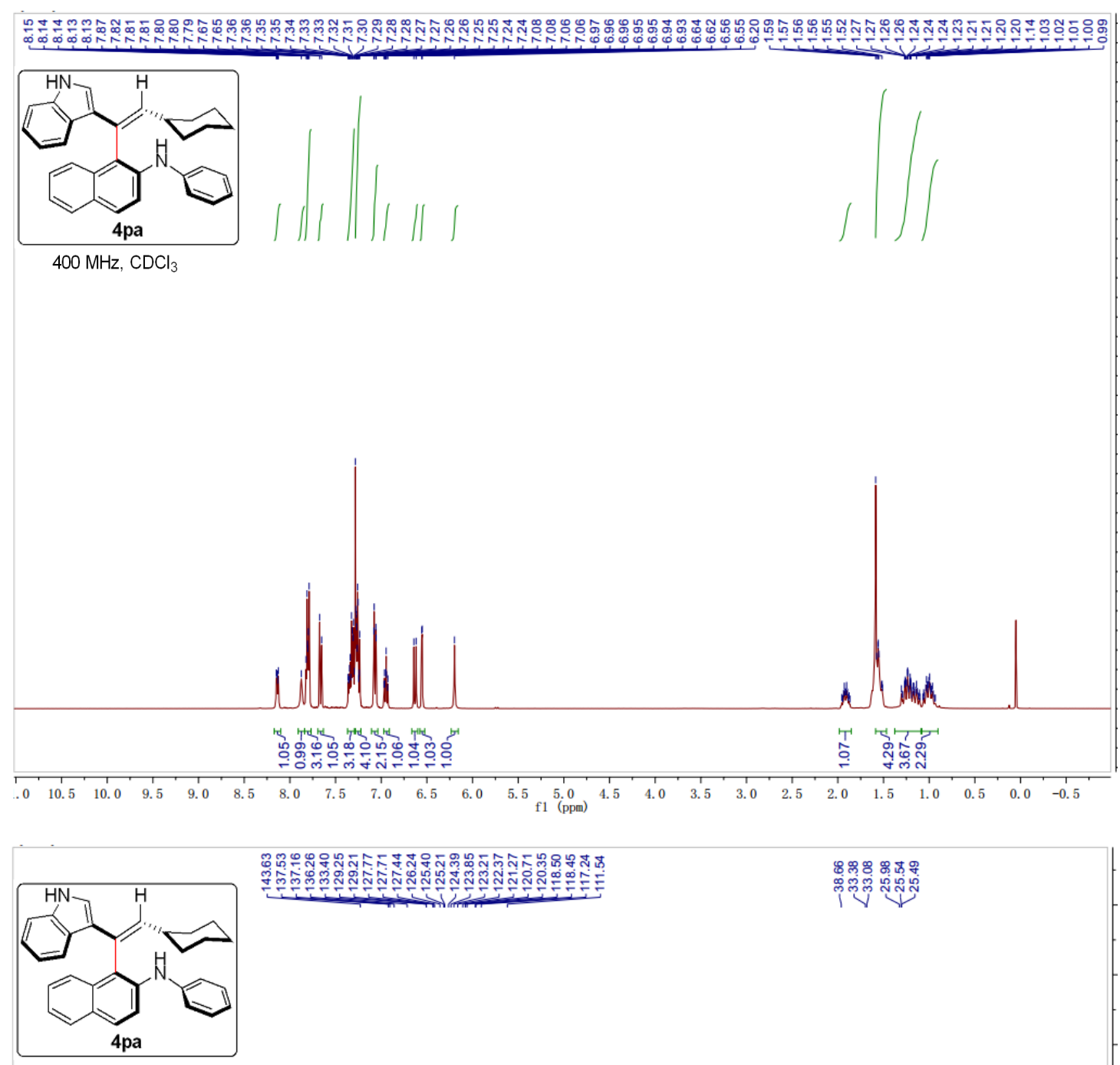

$8 \%$

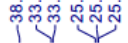

$101 \mathrm{MHz}, \mathrm{CDCl}_{3}$

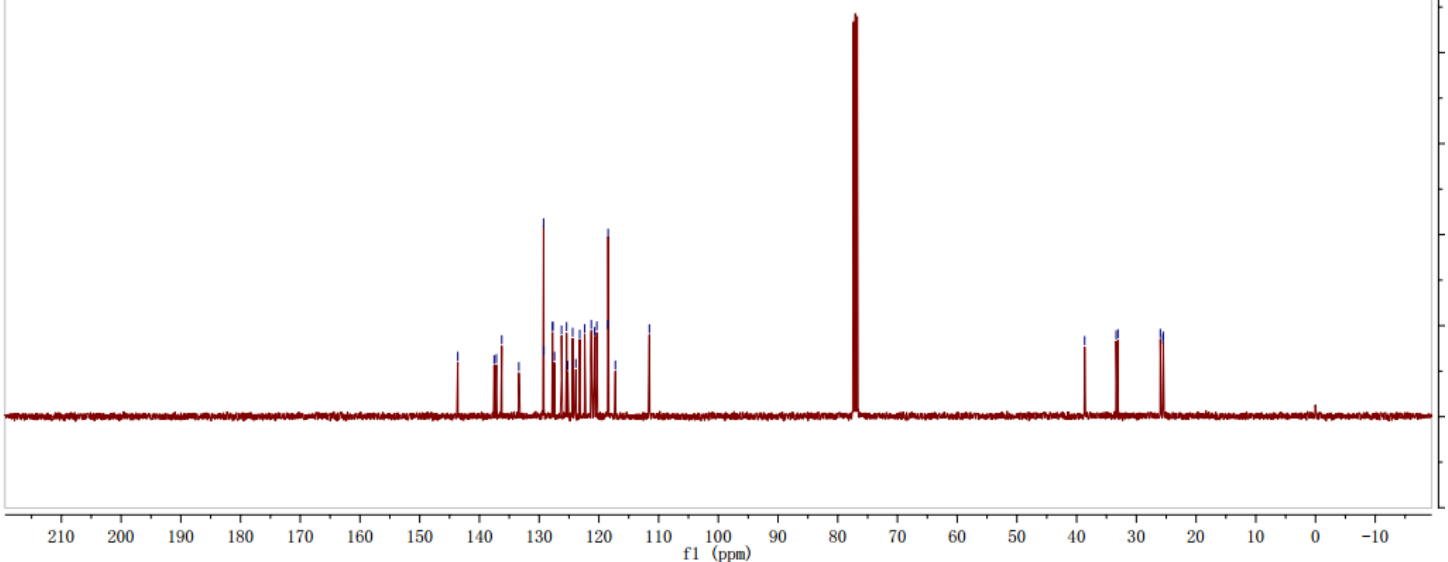




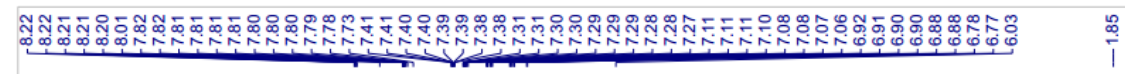

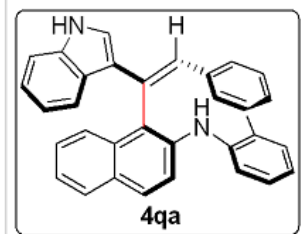

$400 \mathrm{MHz}, \mathrm{CDCl}_{3}$
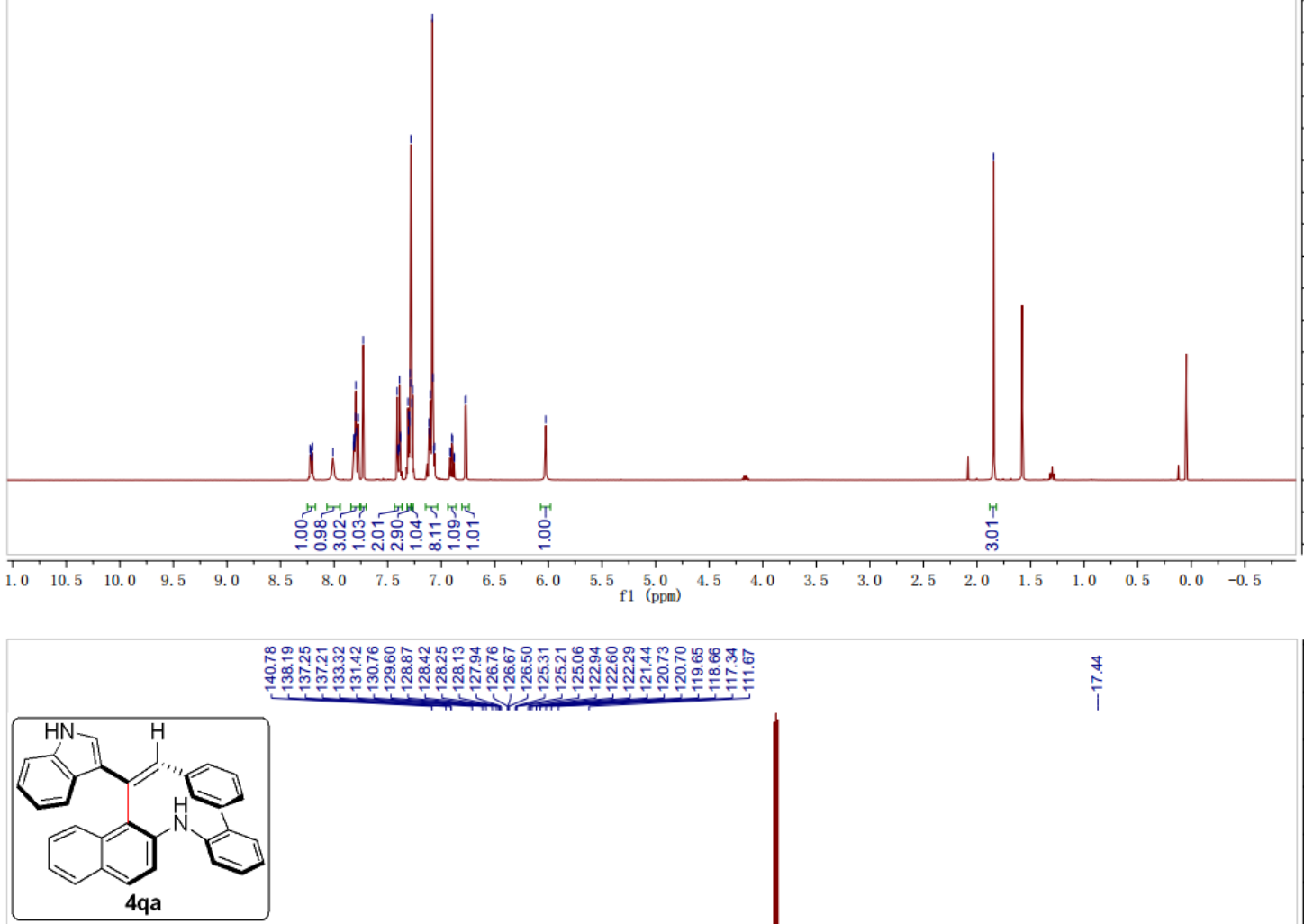

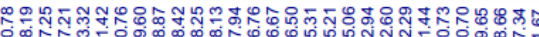

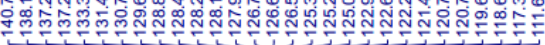

$101 \mathrm{MHz}, \mathrm{CDCl}_{3}$
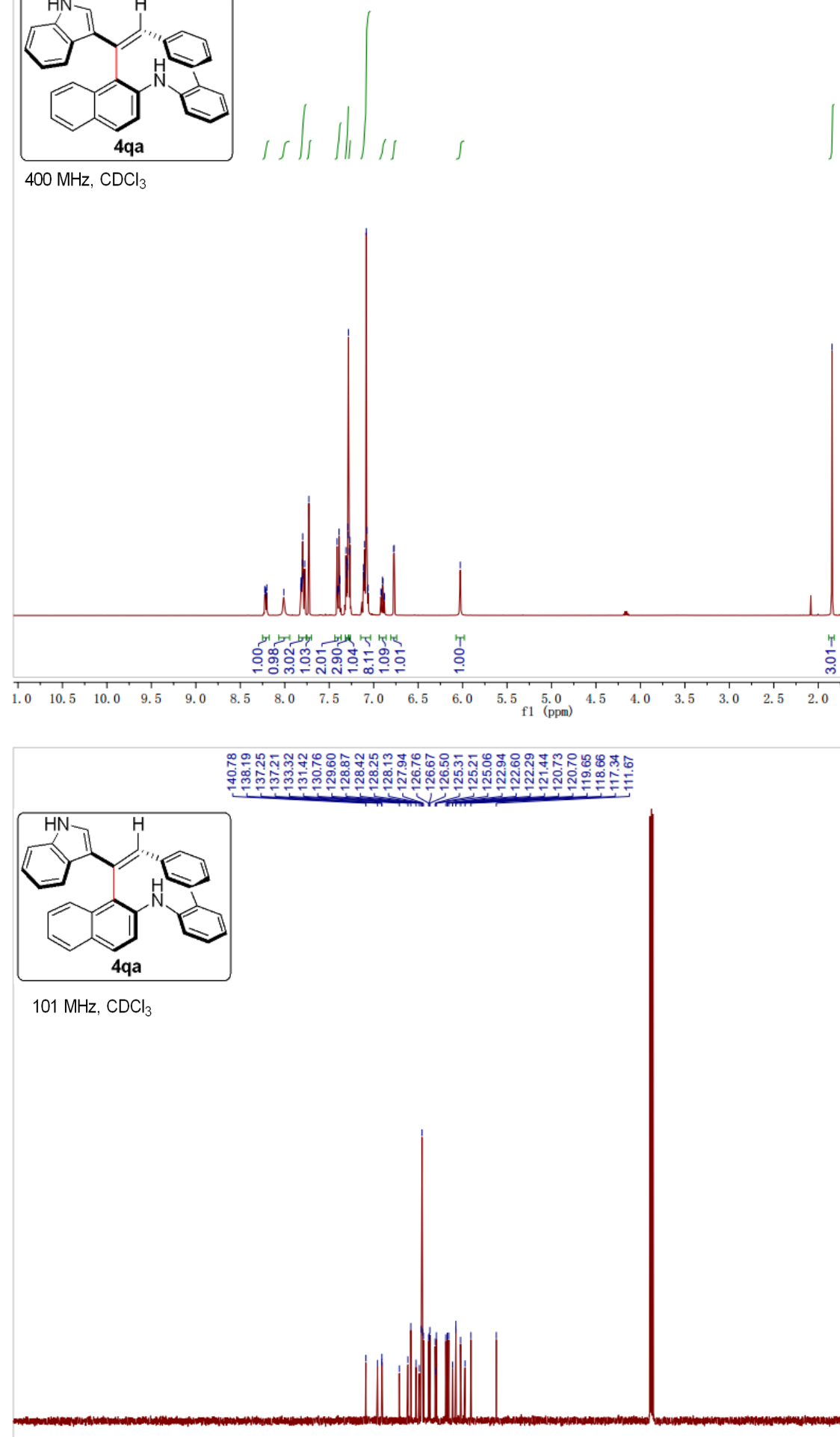

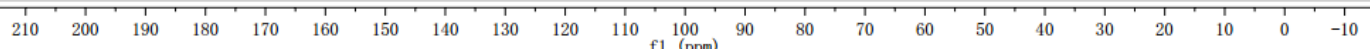



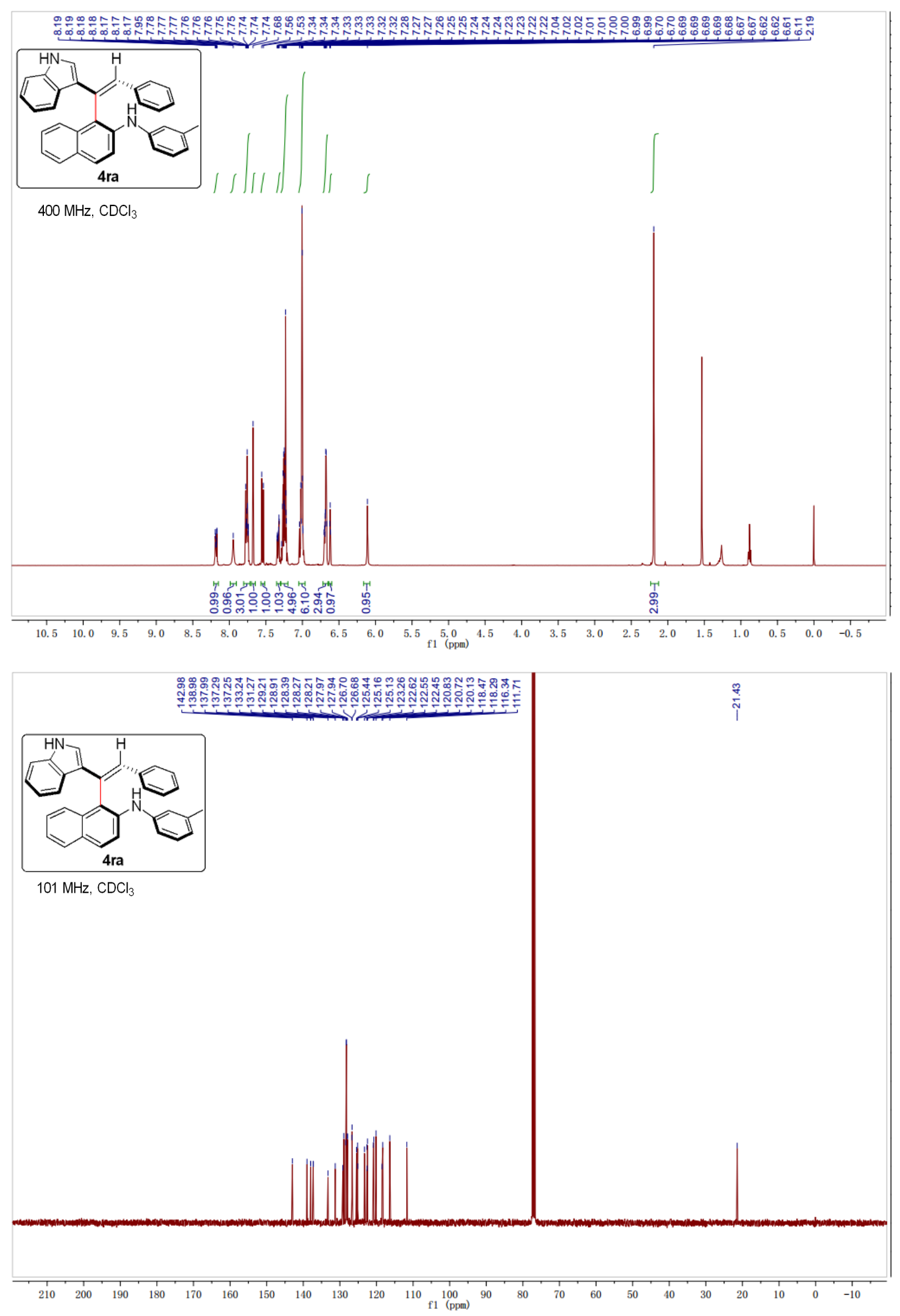

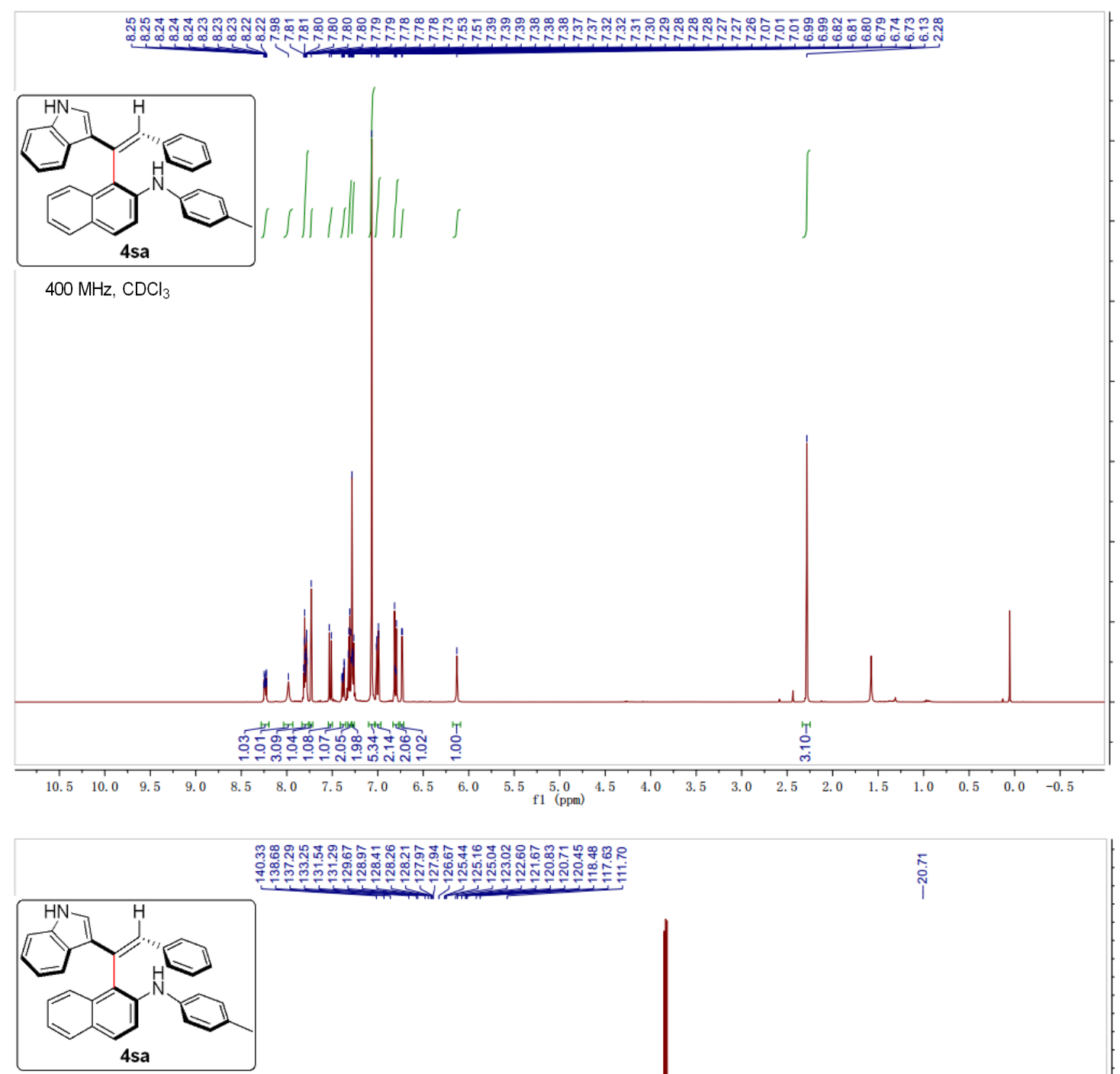

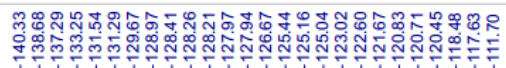

$101 \mathrm{MHz}, \mathrm{CDCl}_{3}$

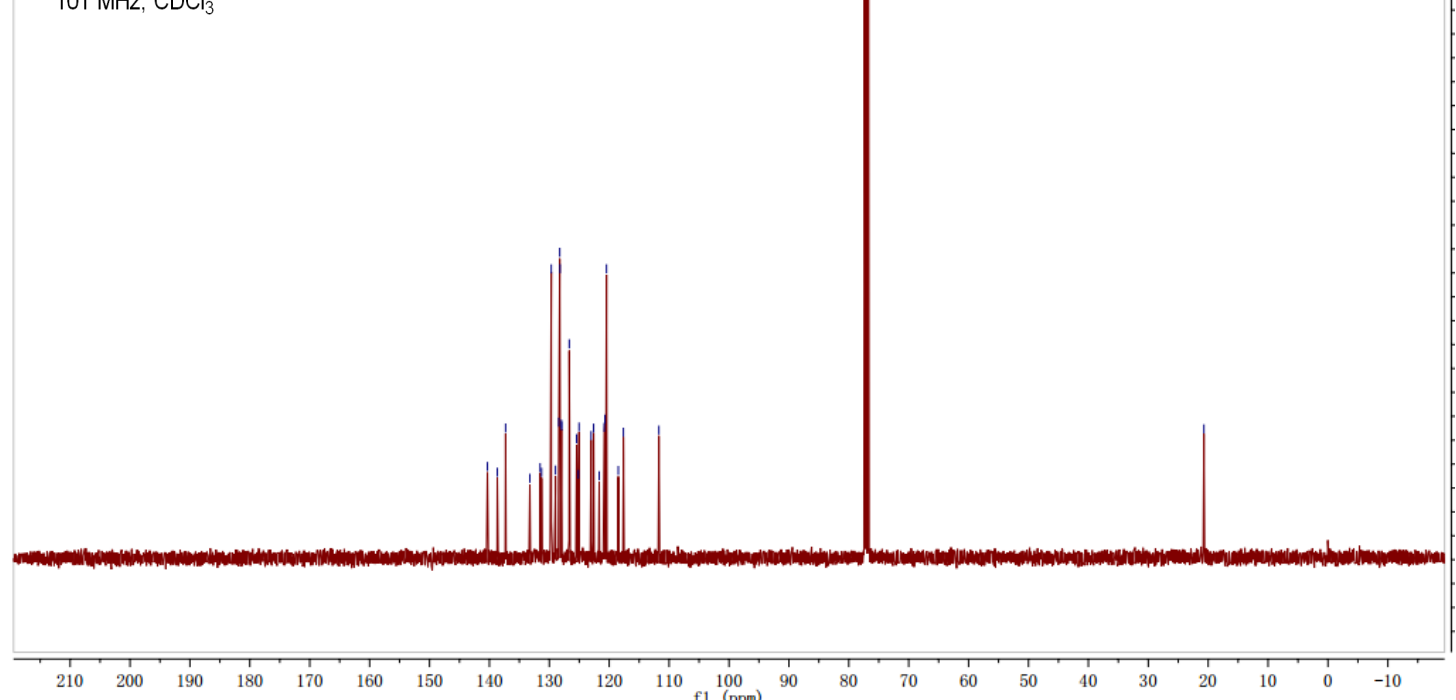



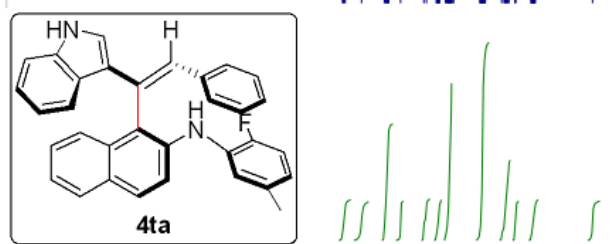

$400 \mathrm{MHz}, \mathrm{CDCl}_{3}$
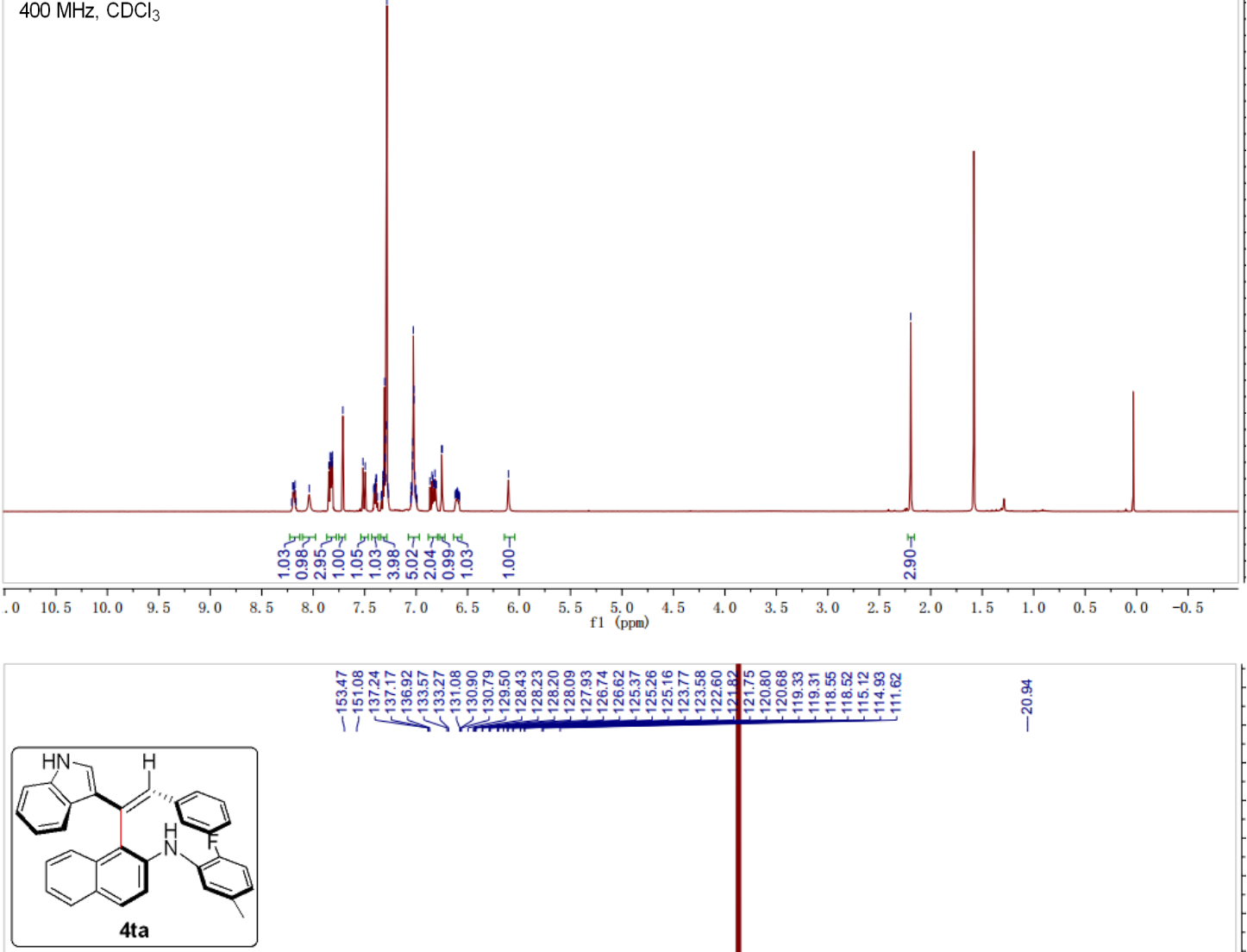

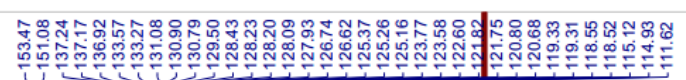

$101 \mathrm{MHz}, \mathrm{CDCl}_{3}$
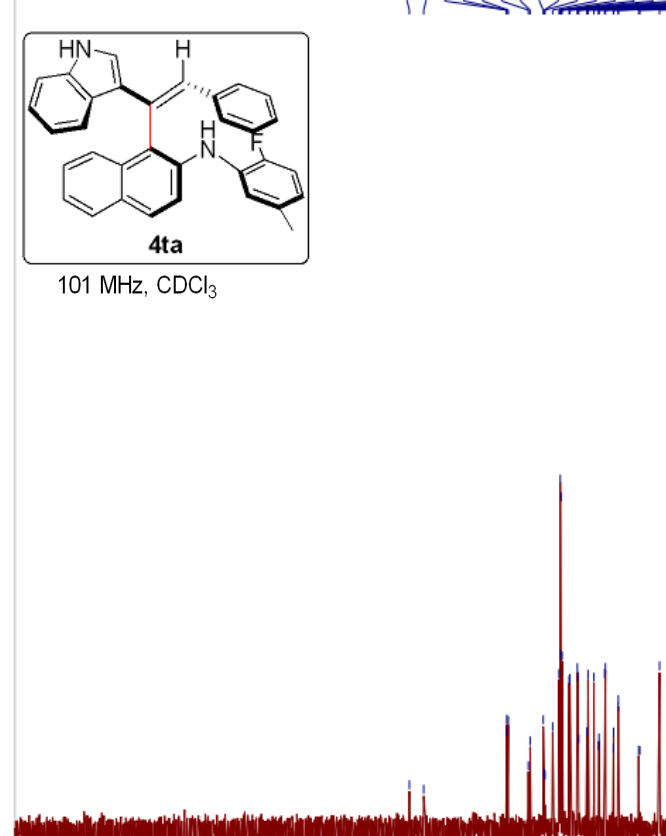

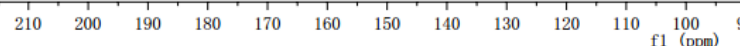




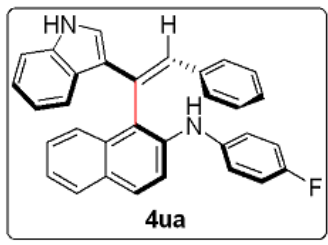

$400 \mathrm{MHz}, \mathrm{CDCl}_{3}$

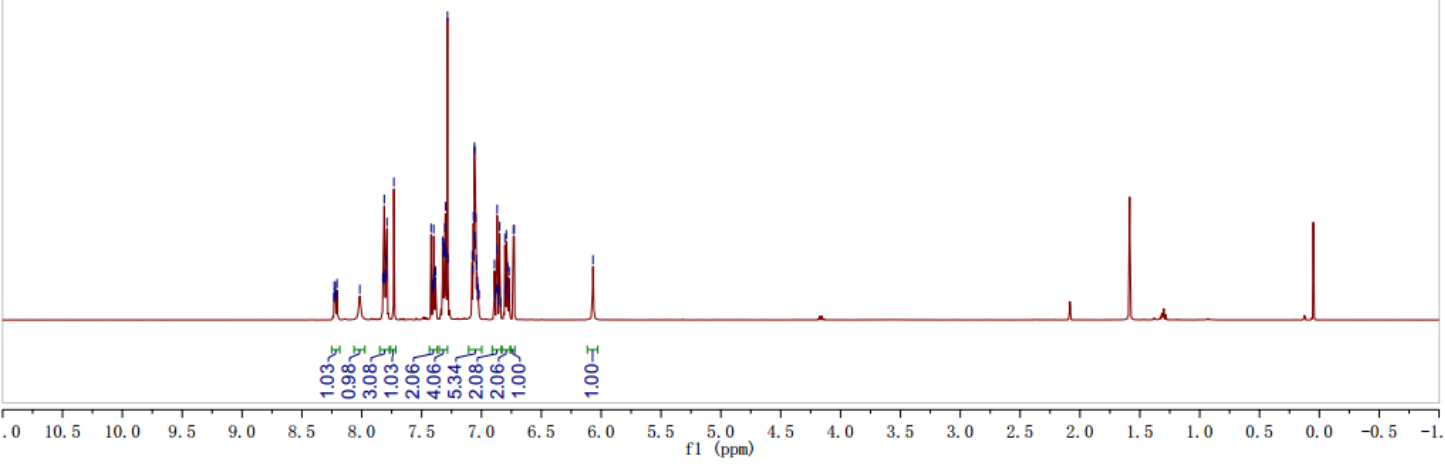

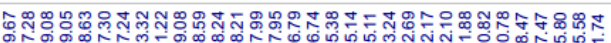

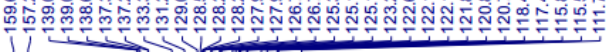

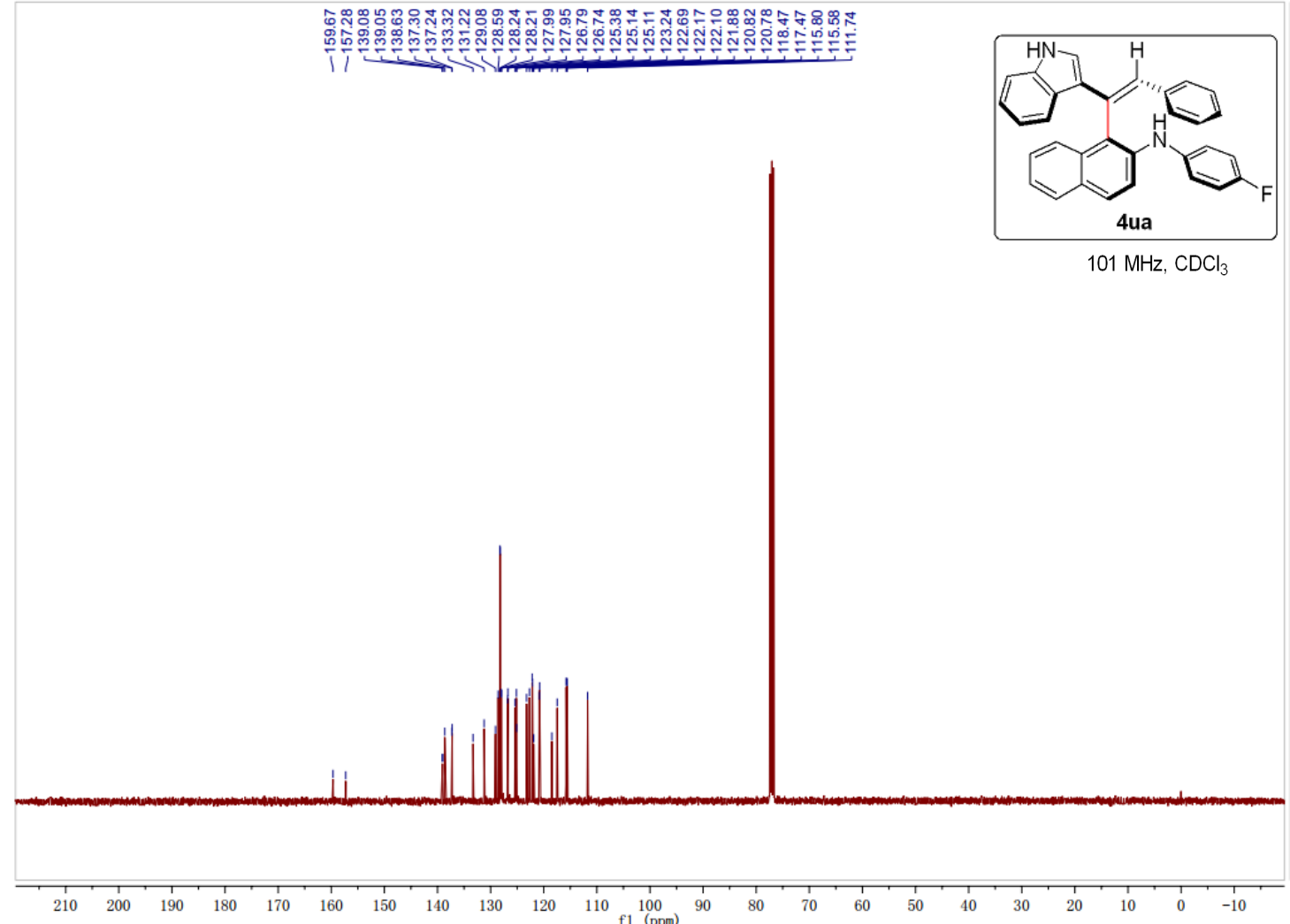



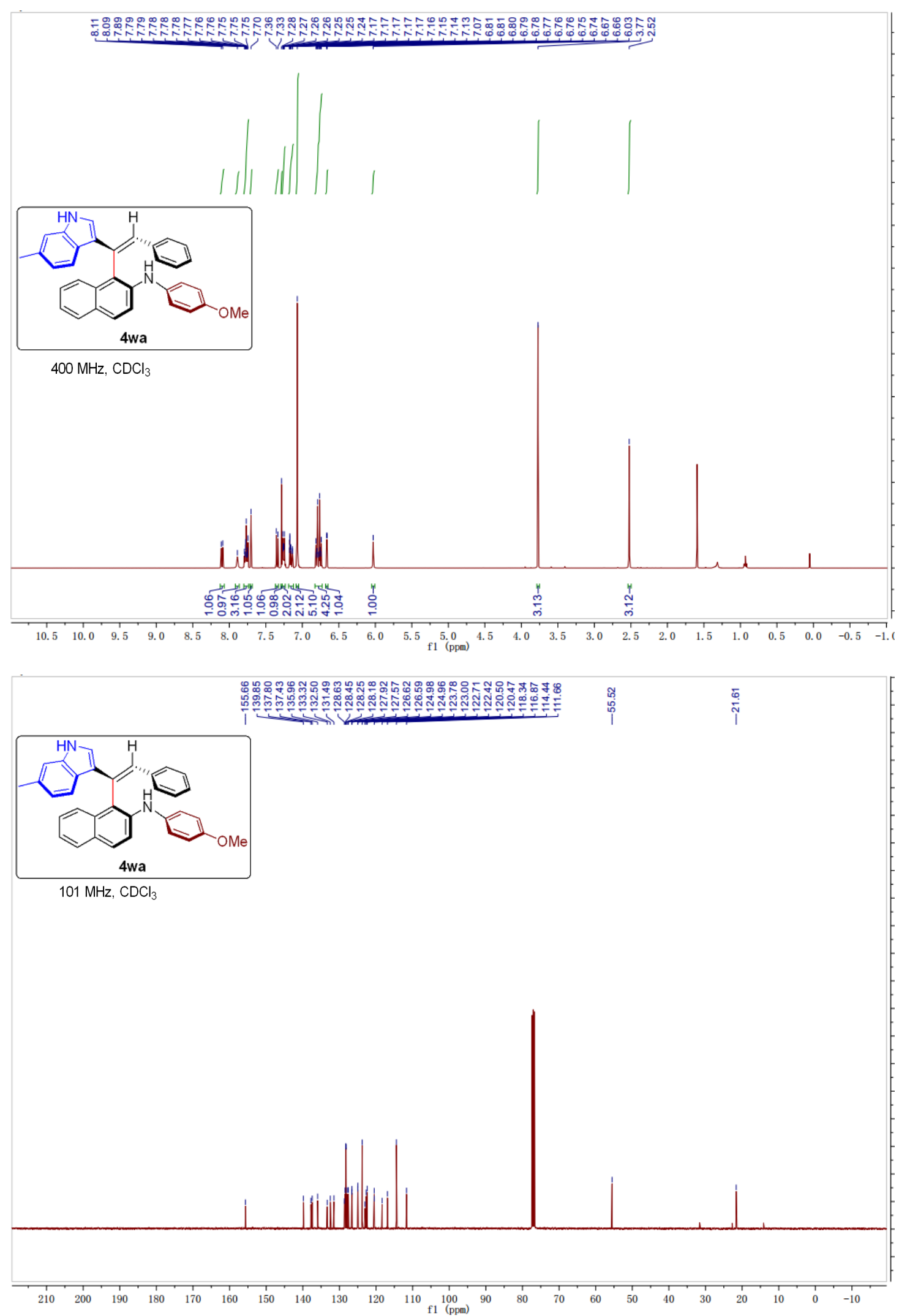


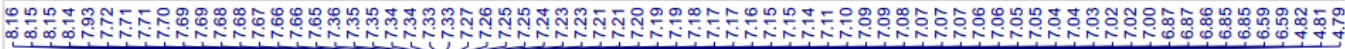

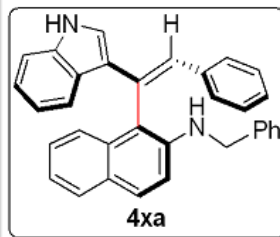

$400 \mathrm{MHz}, \mathrm{CDCl}_{3}$
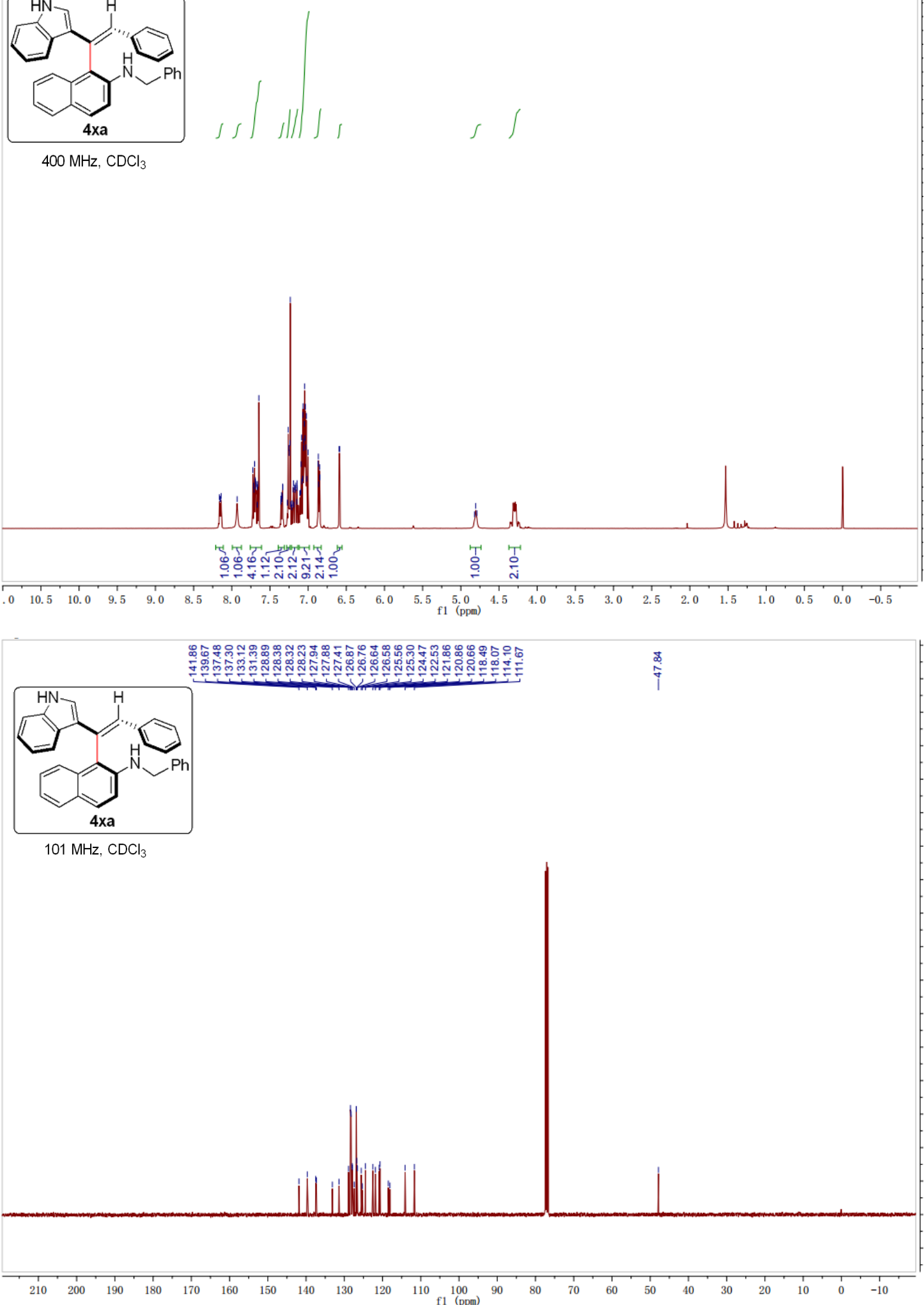


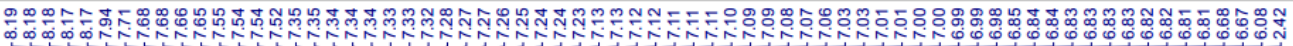

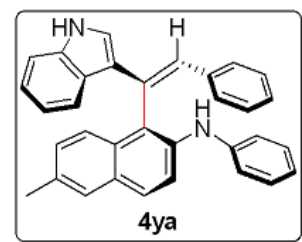

$|d| 1||$

$400 \mathrm{MHz}, \mathrm{CDCl}_{3}$

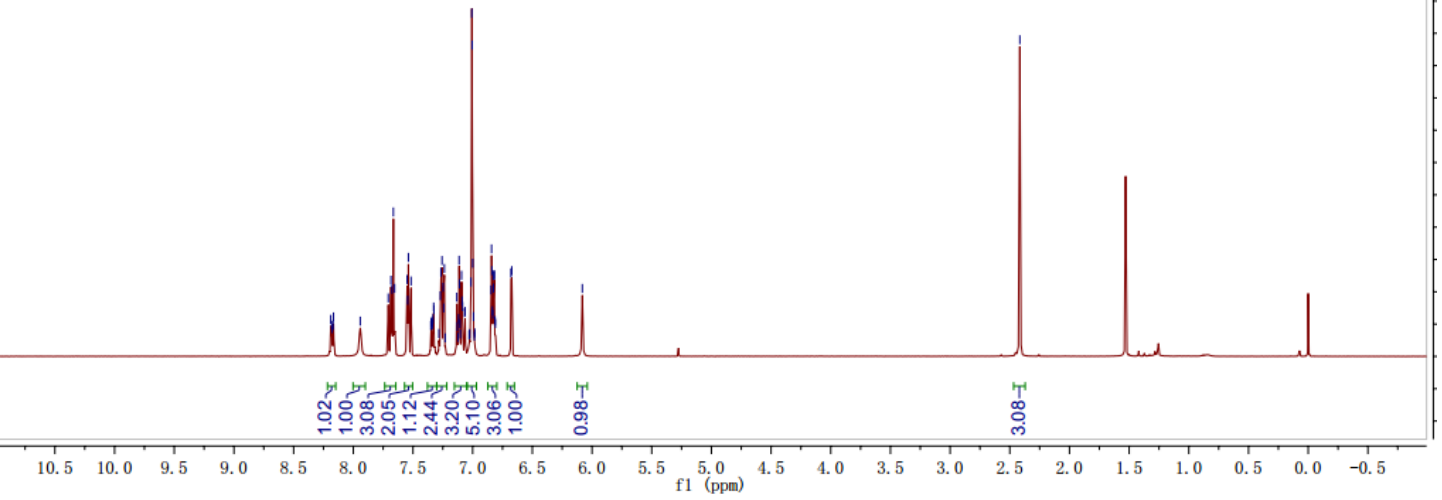

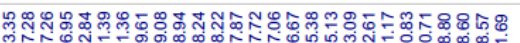

7.

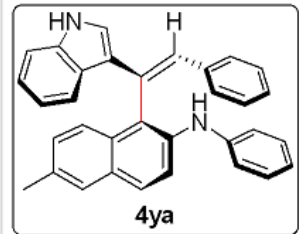

$101 \mathrm{MHz}, \mathrm{CDCl}_{3}$

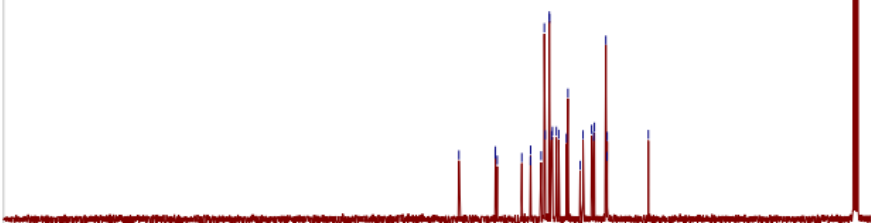

$\begin{array}{lllllllllllll}210 & 200 & 190 & 180 & 170 & 160 & 150 & 140 & 130 & 120 & 110 & 100 \\ \mathrm{fl} 1 & (\mathrm{pmm}) & 1\end{array}$ 


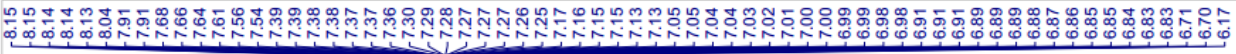
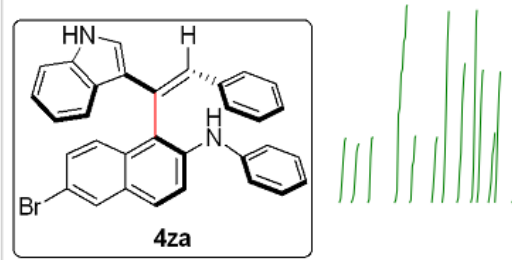

$400 \mathrm{MHz}, \mathrm{CDCl}_{3}$
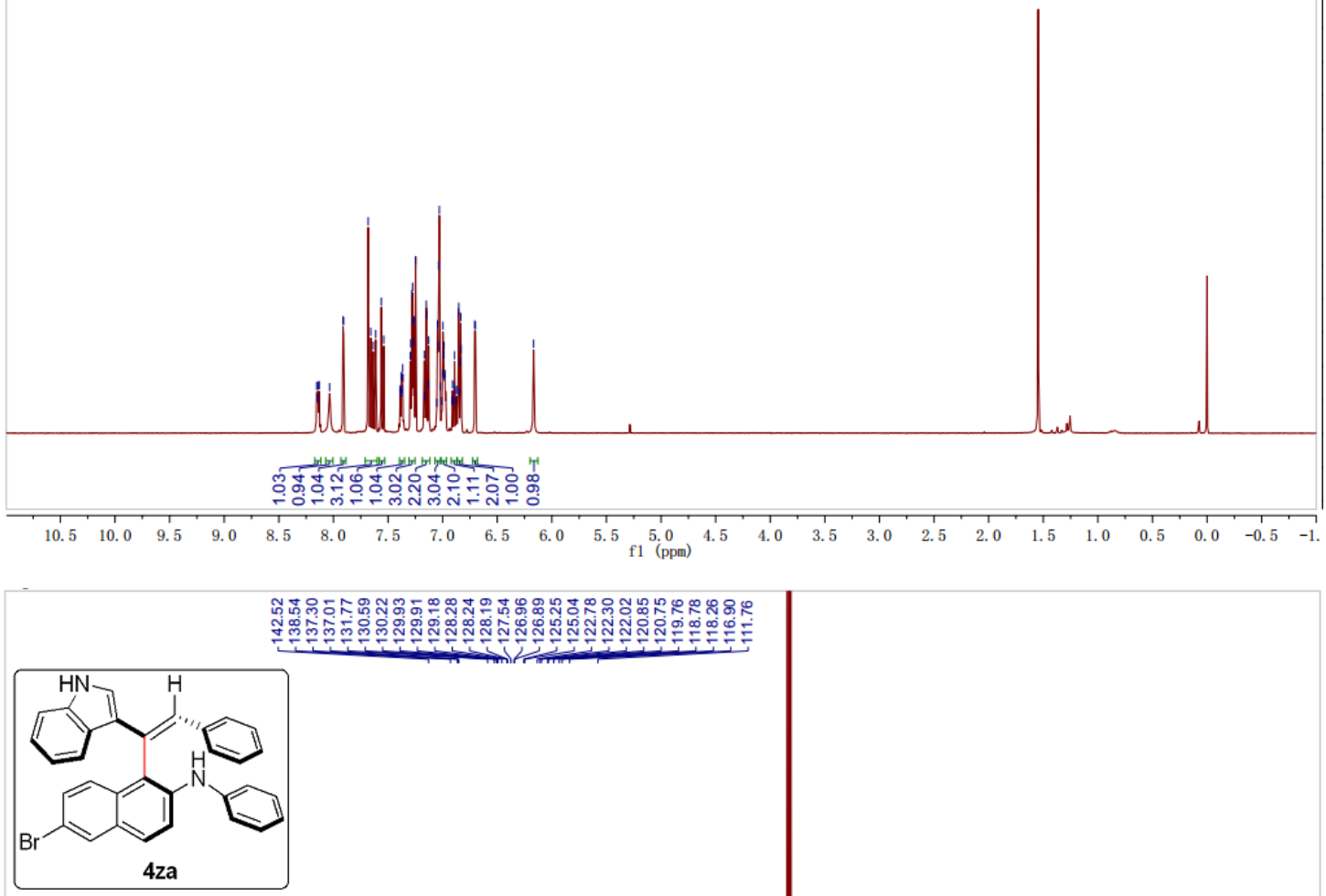

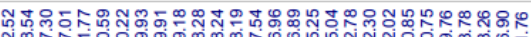

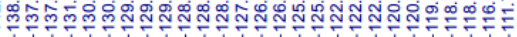

$101 \mathrm{MHz}, \mathrm{CDCl}_{3}$

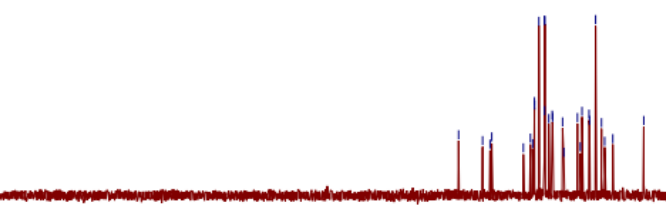

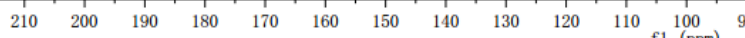



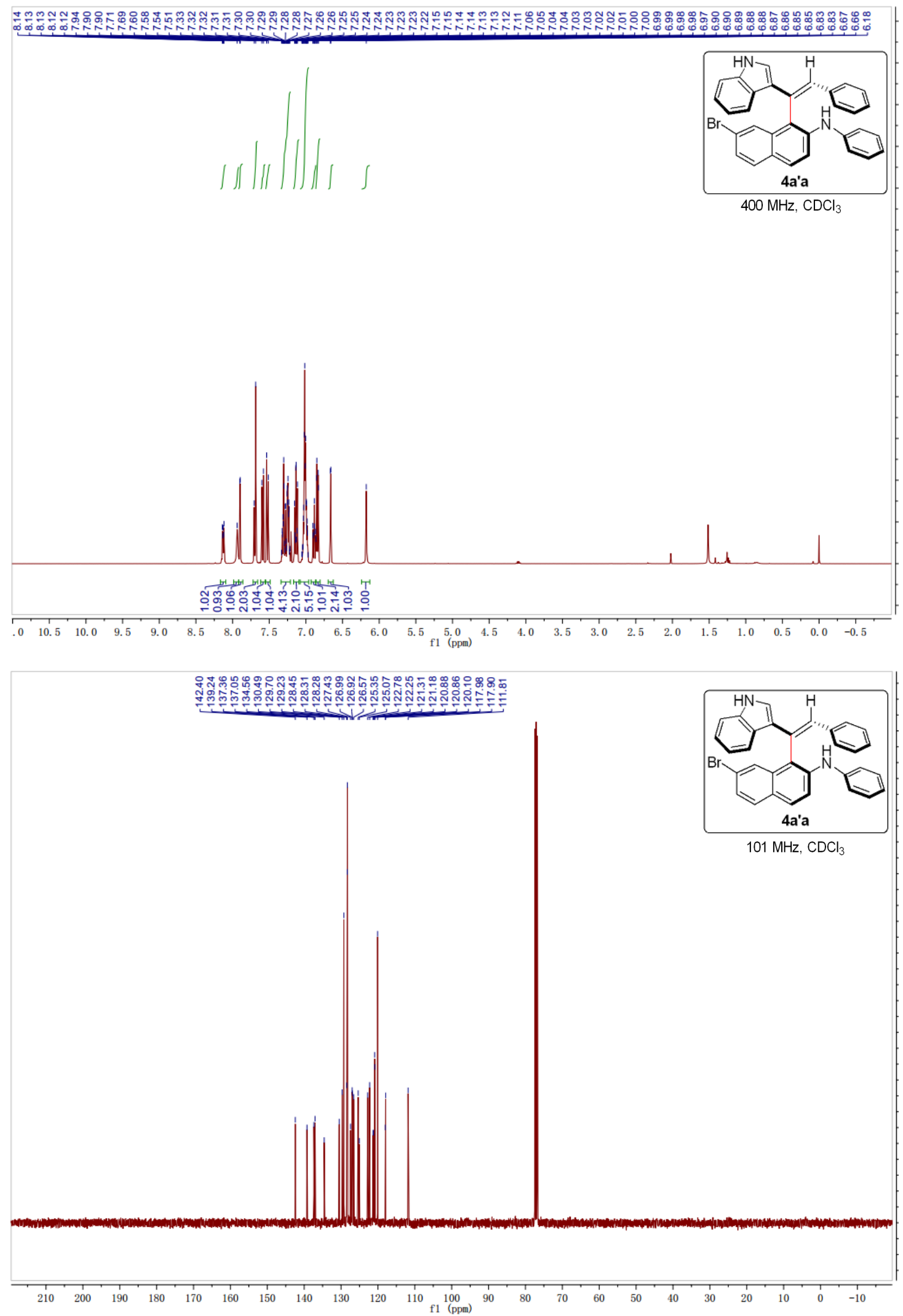

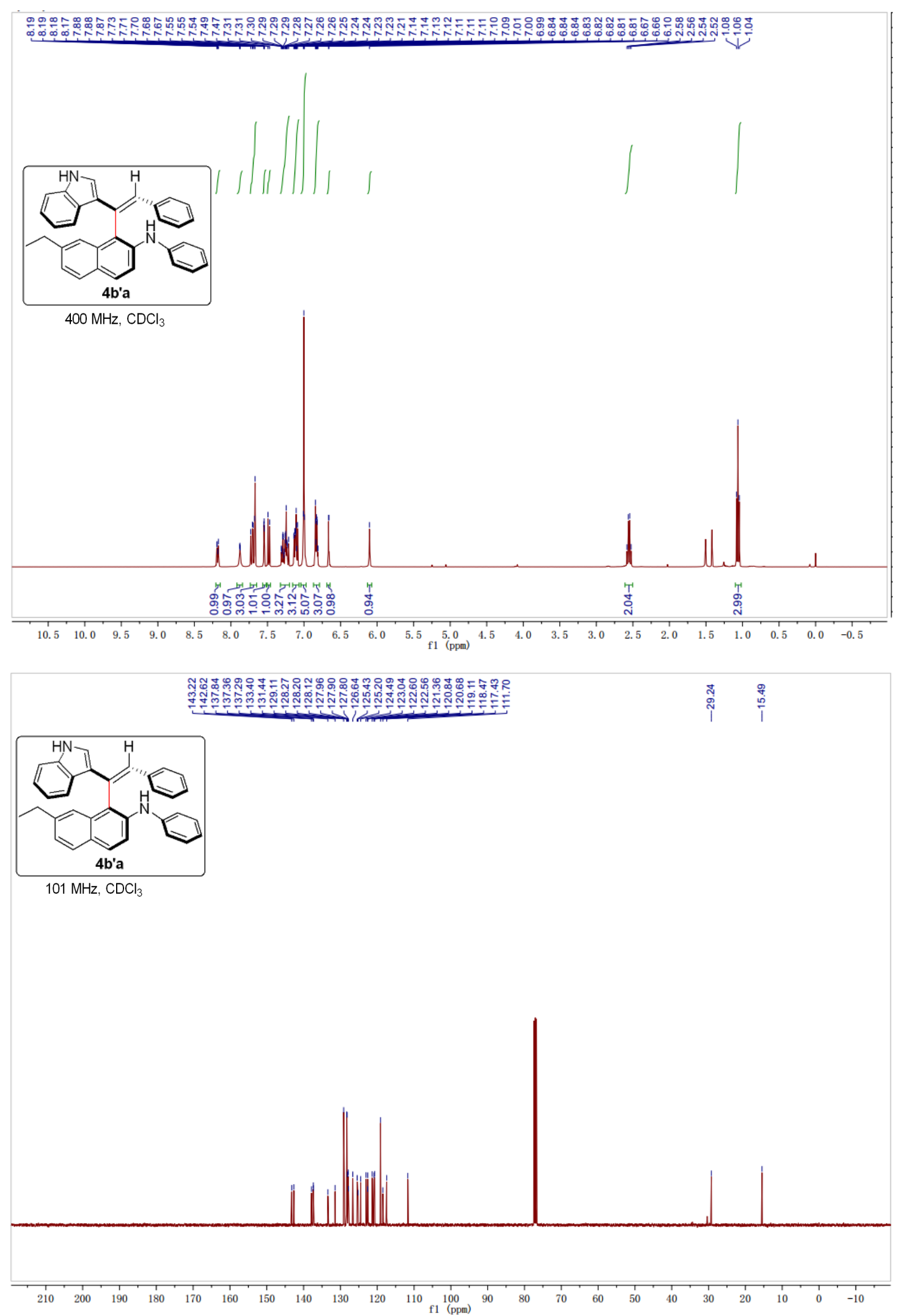

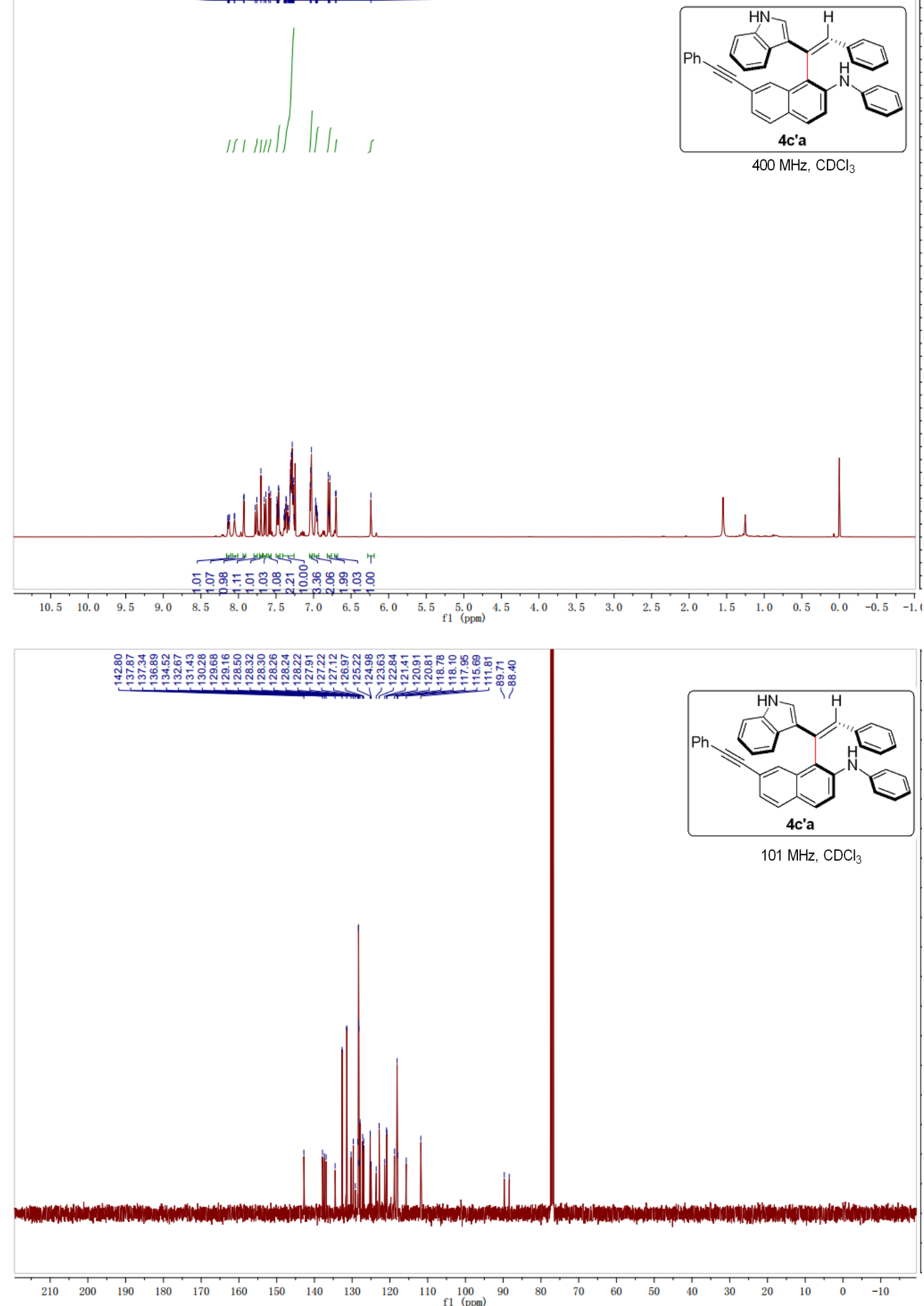


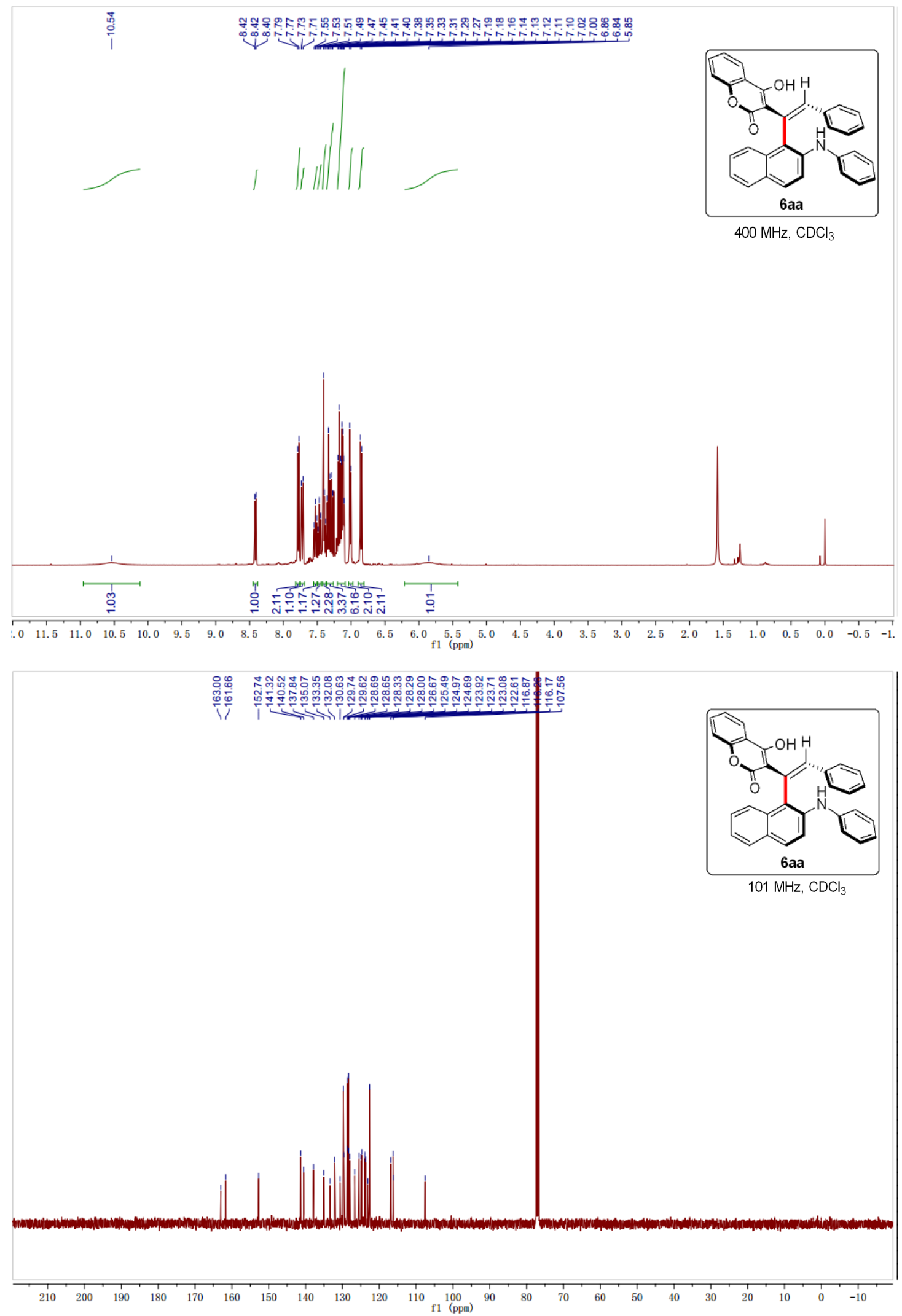




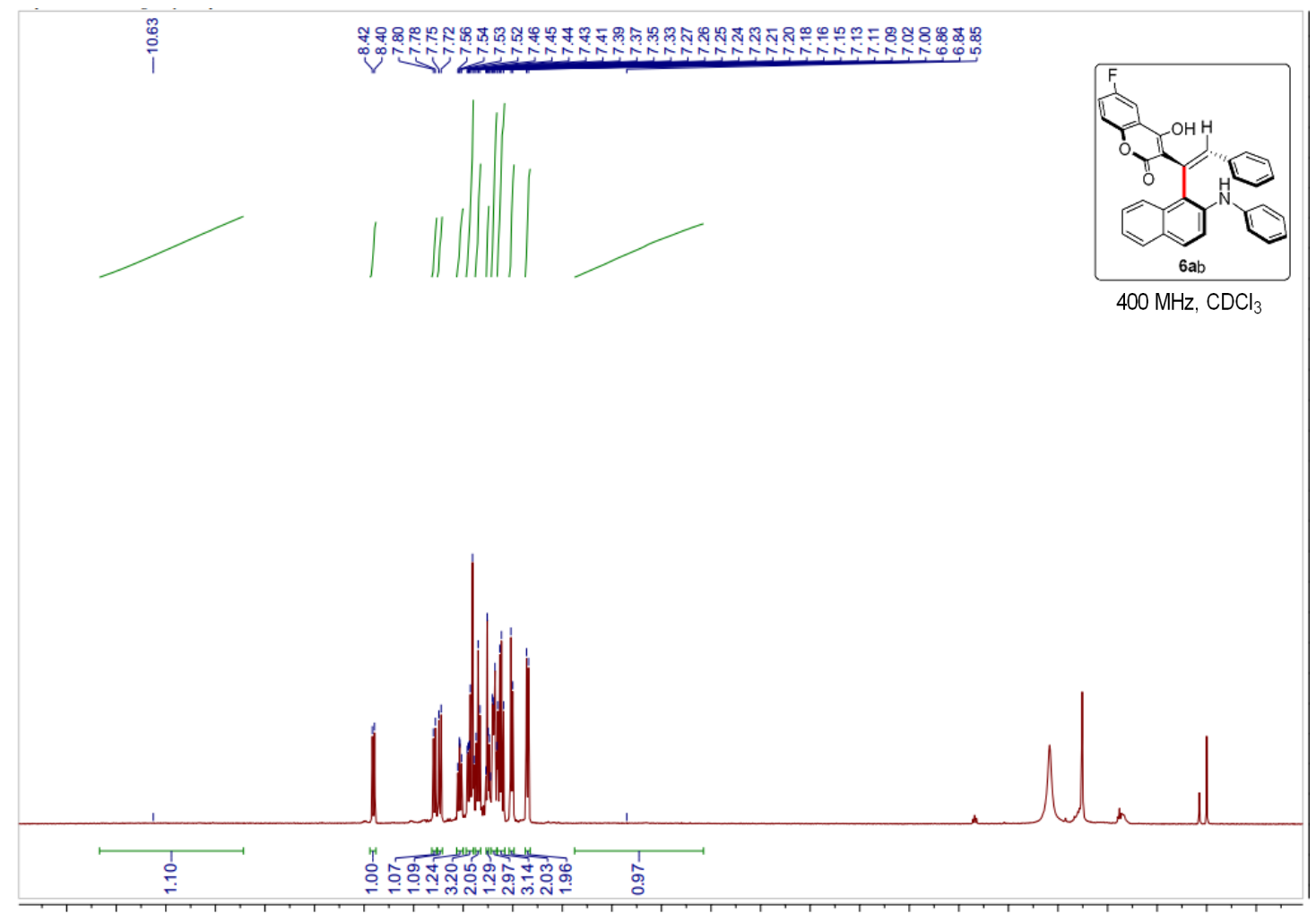

$\begin{array}{lllllllllllllllllllllllll}11.5 & 11.0 & 10.5 & 10.0 & 9.5 & 9.0 & 8.5 & 8.0 & 7.5 & 7.0 & 6.5 & 6.0 & 5.5 & 5.0 & 4.5 & 4.0 & 3.5 & 3.0 & 2.5 & 2.0 & 1.5 & 1.0 & 0.5 & 0.0 & -0.5\end{array}$

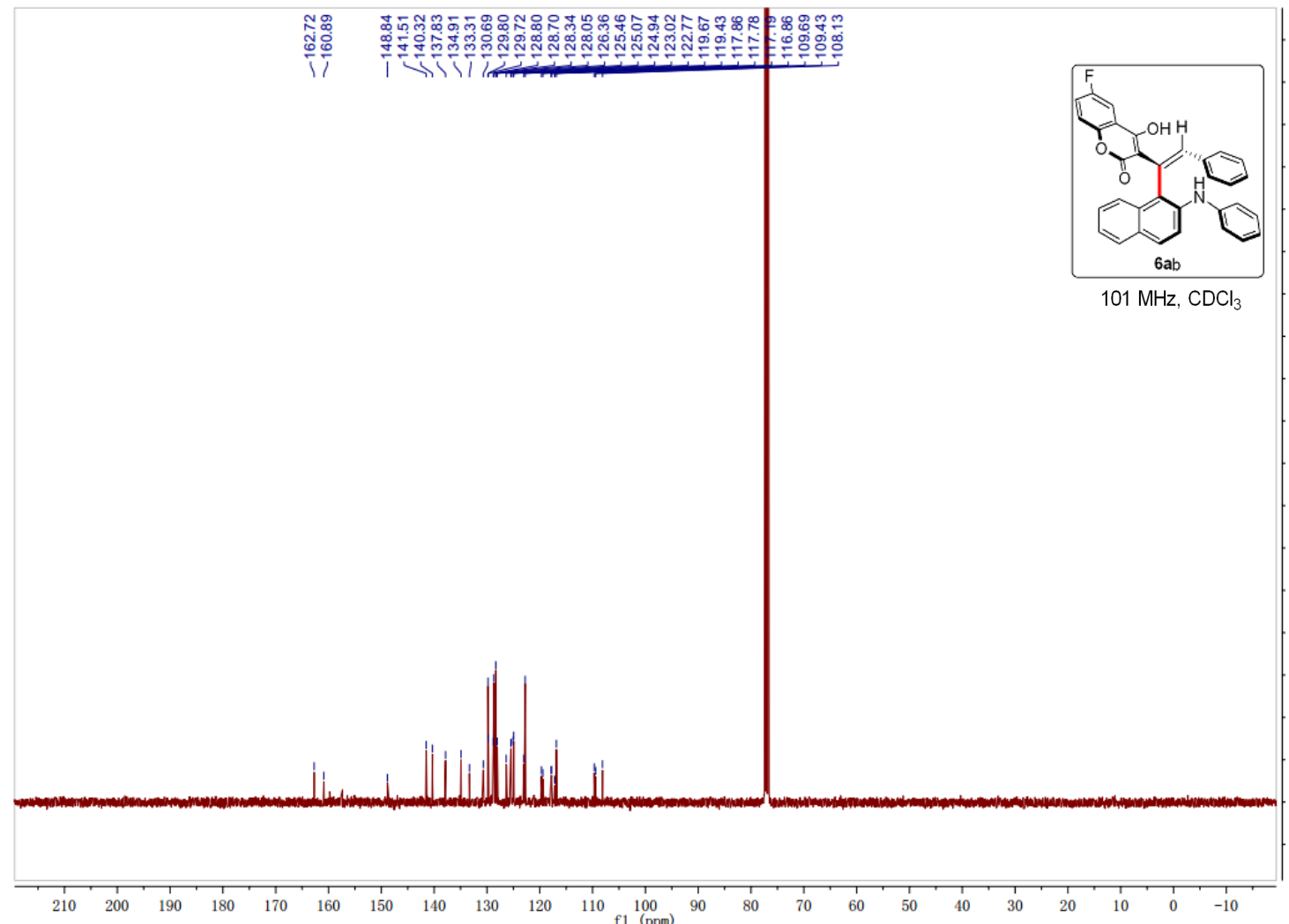



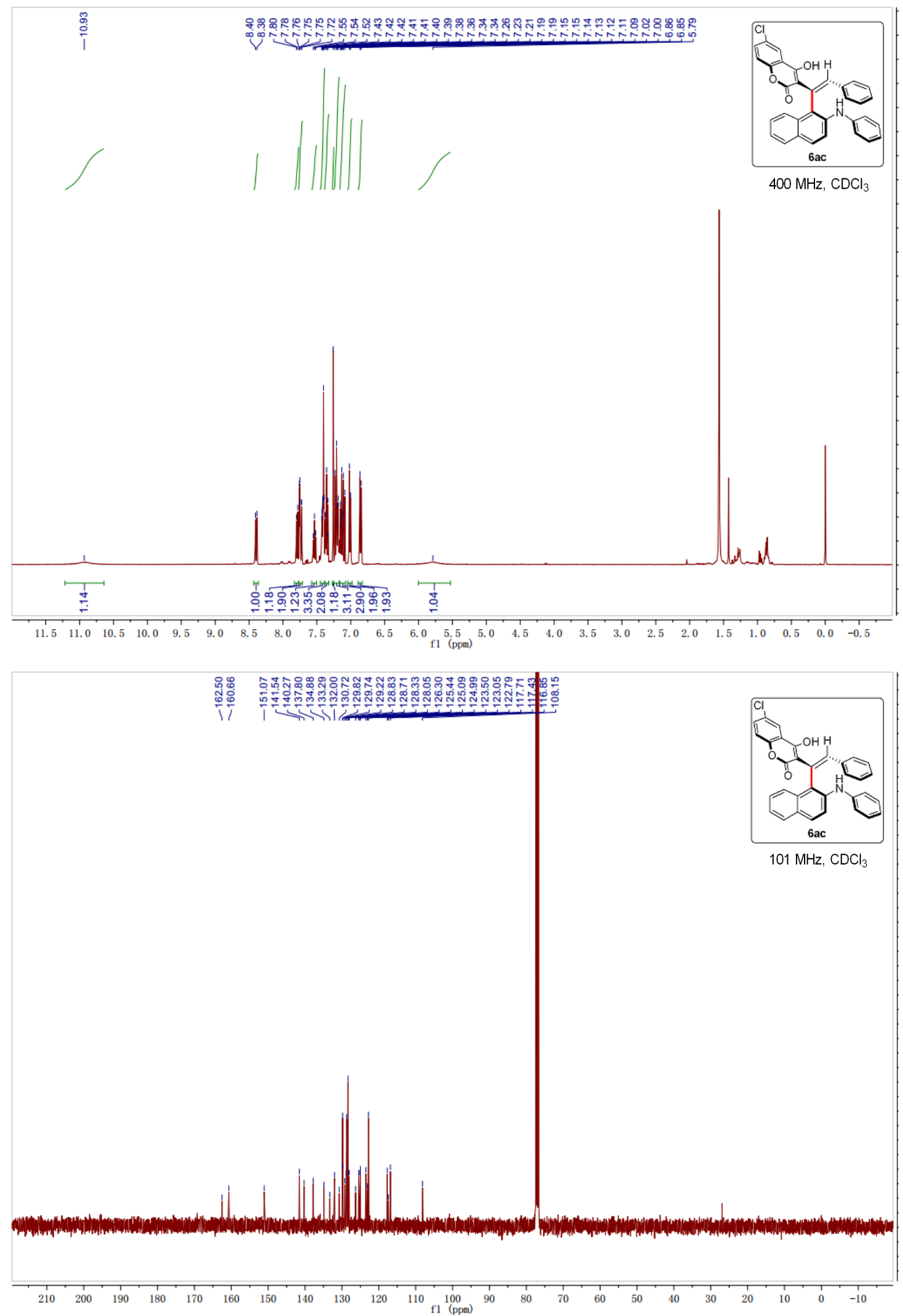

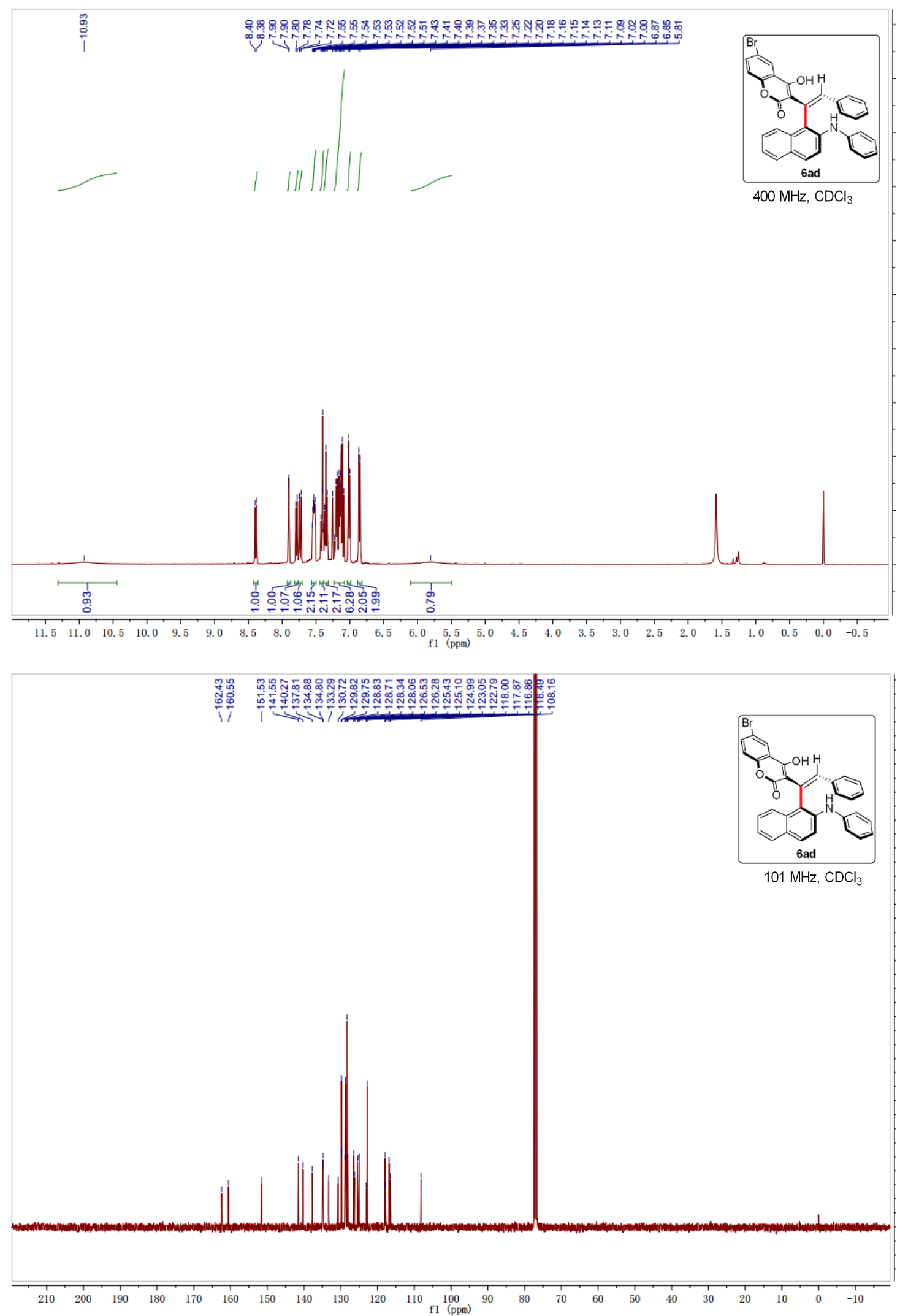


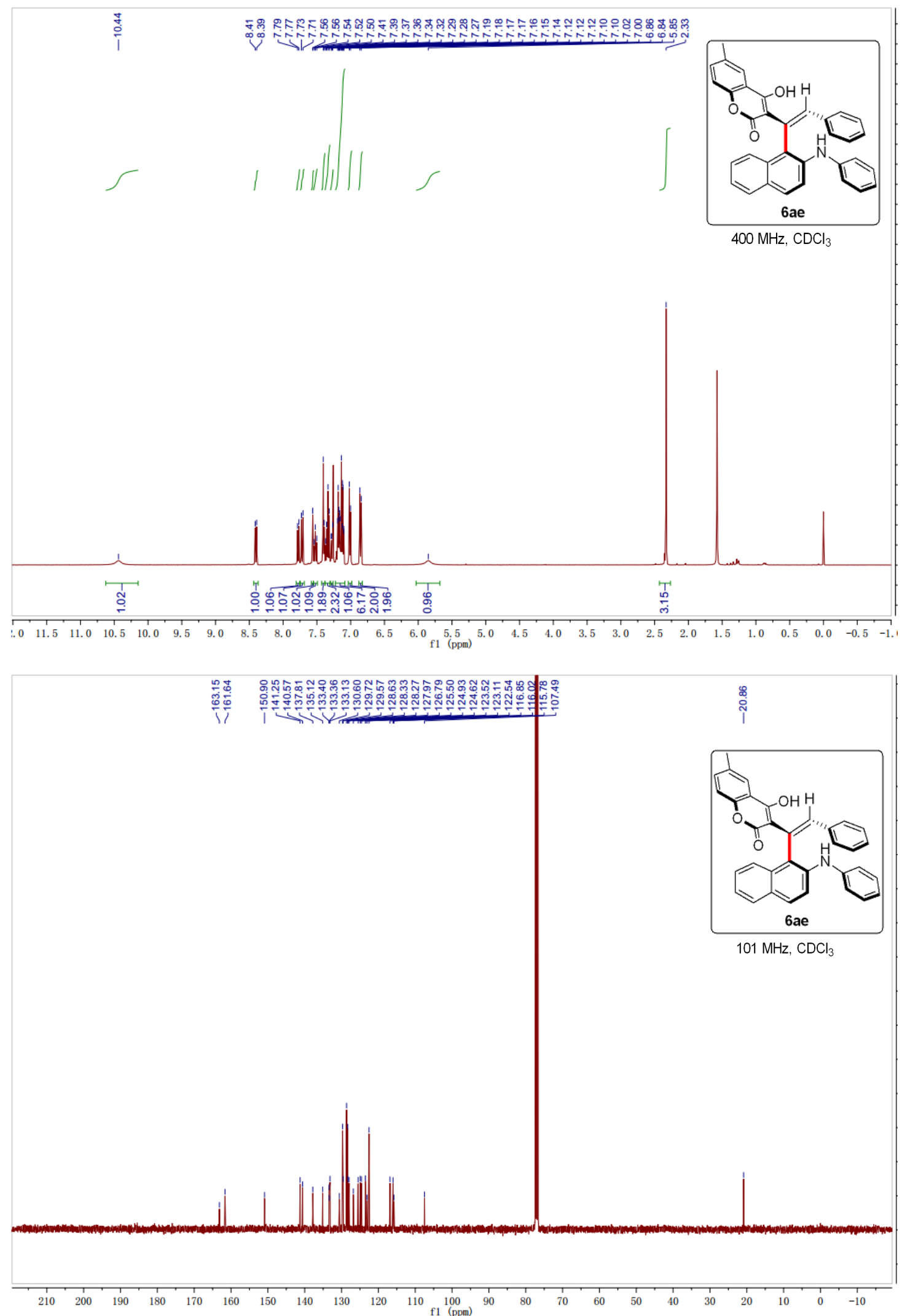



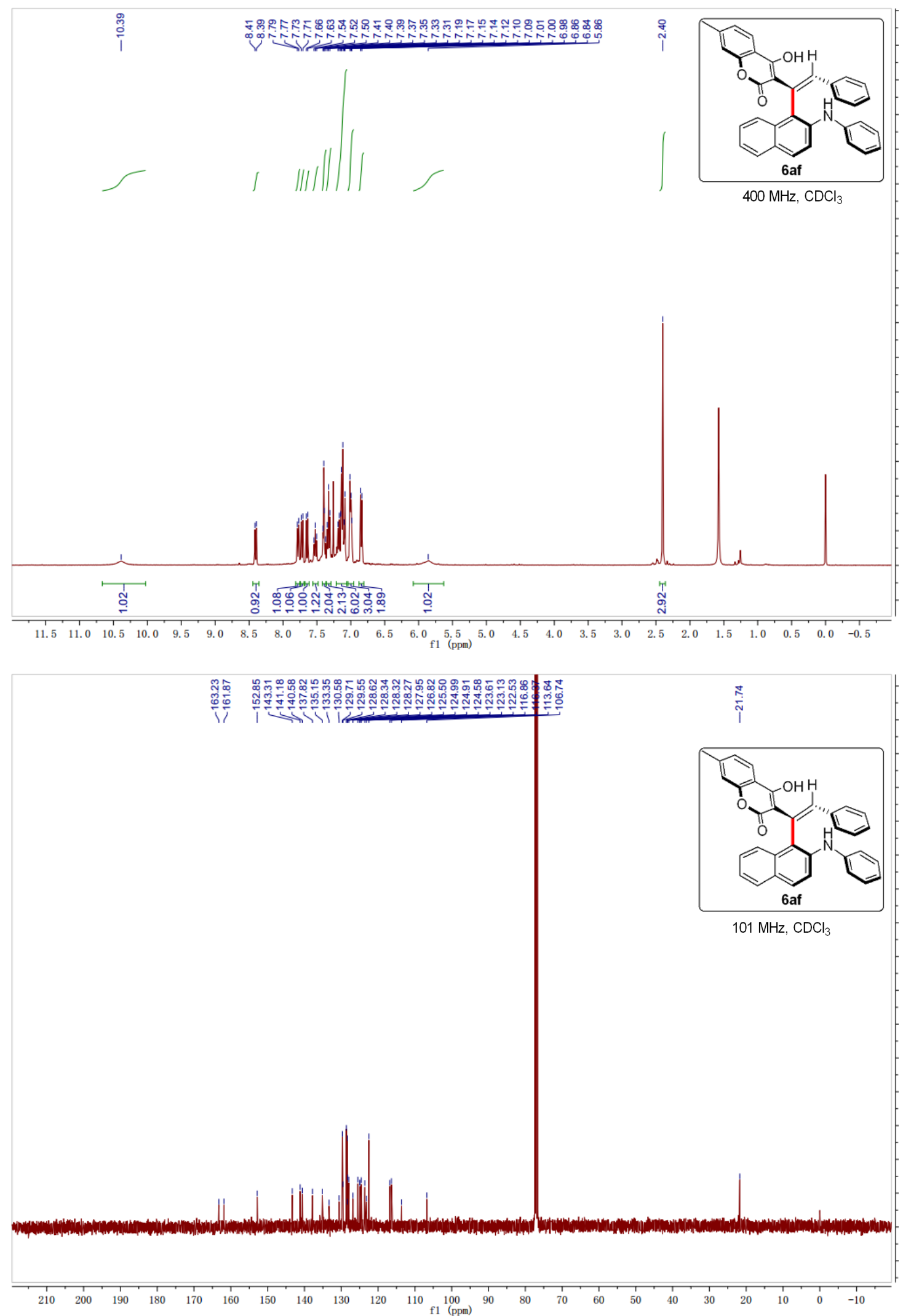

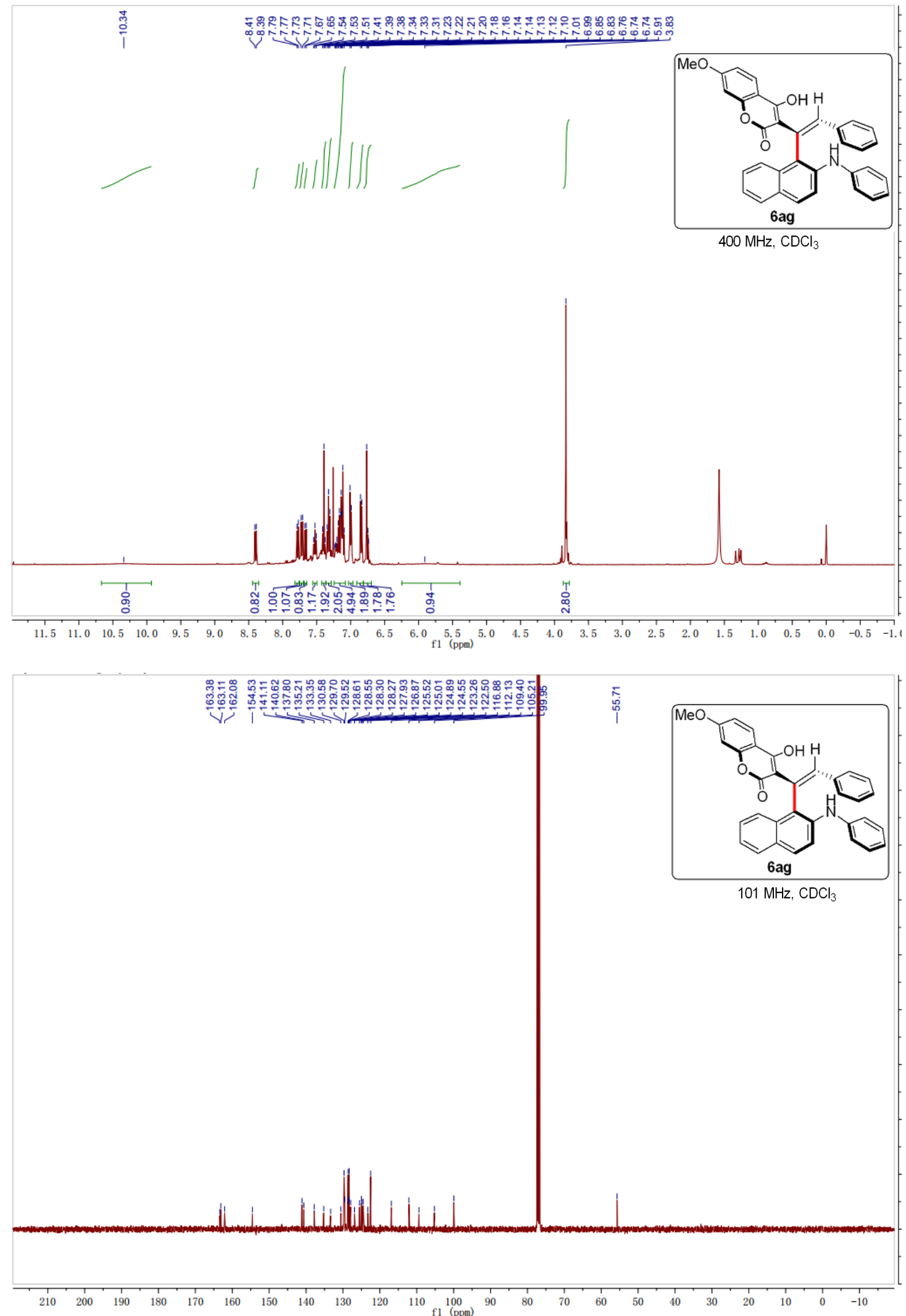

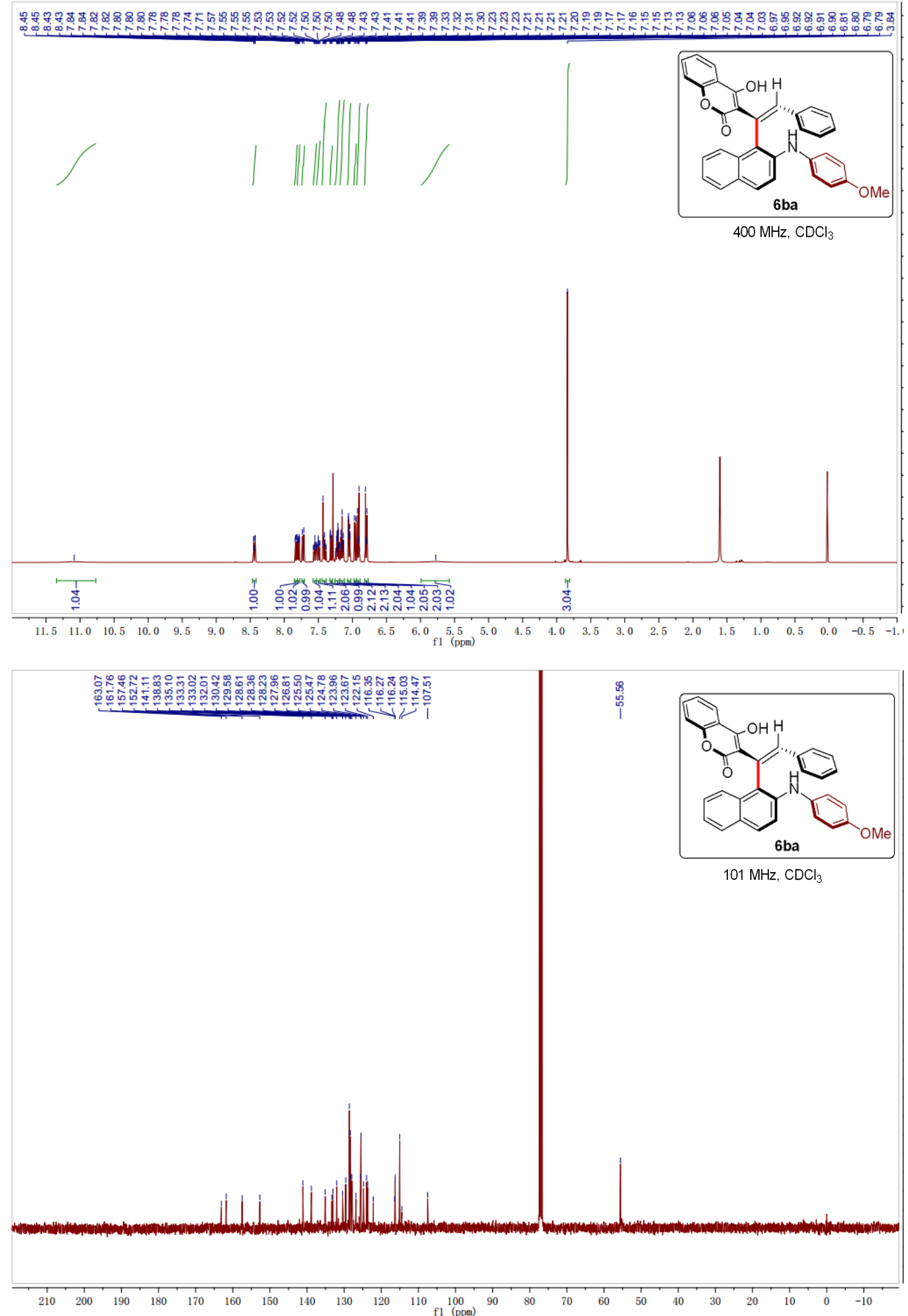

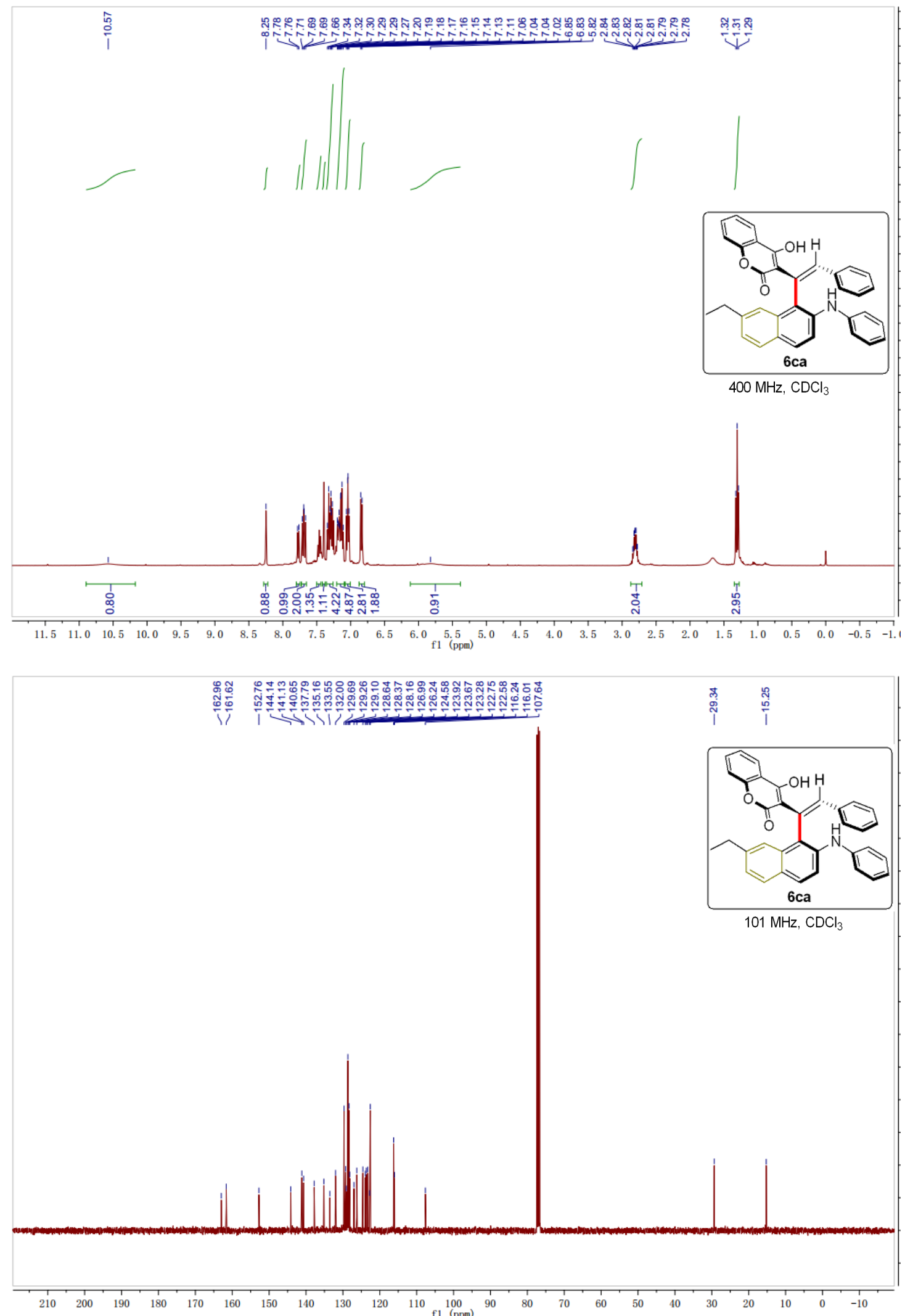

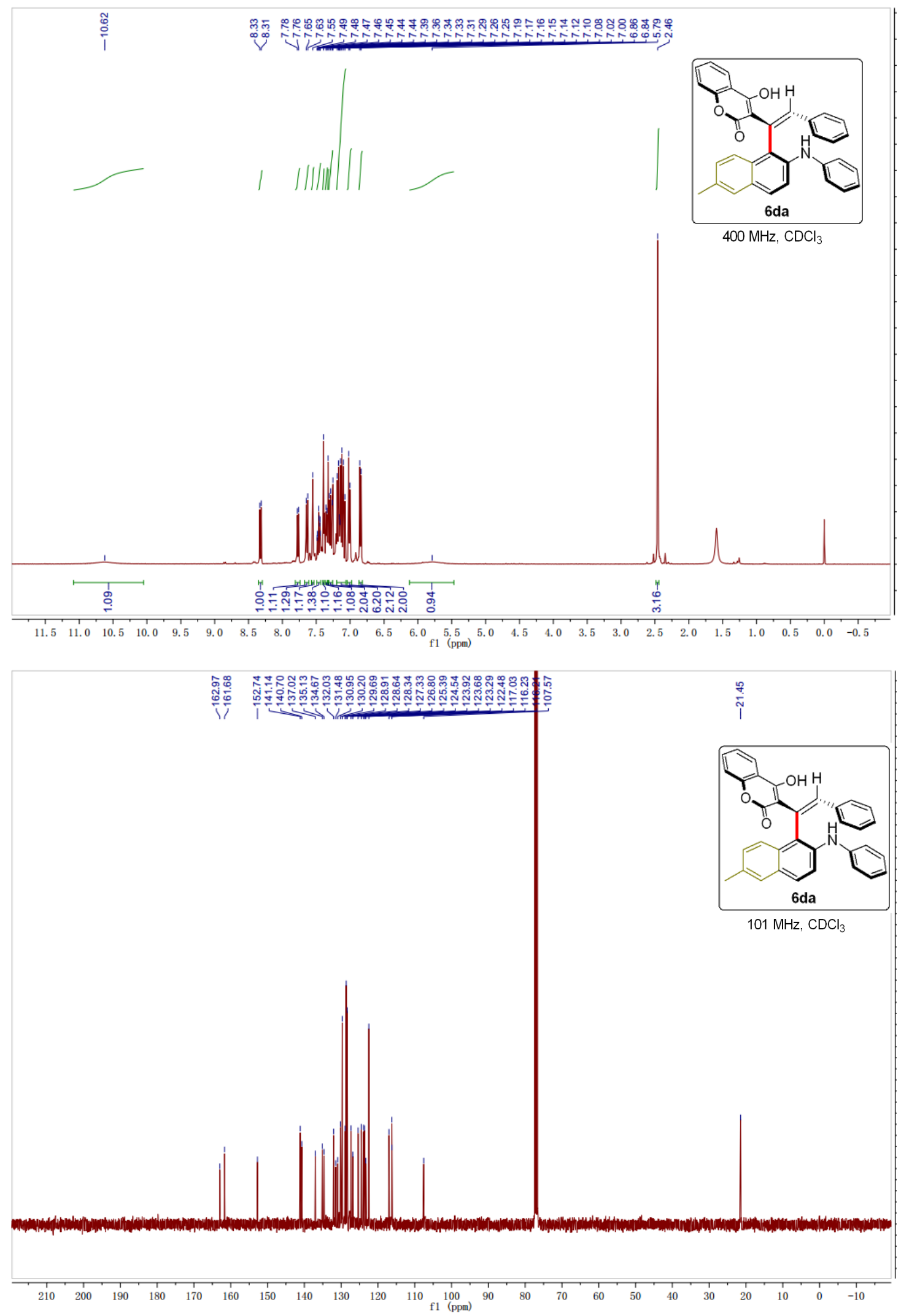

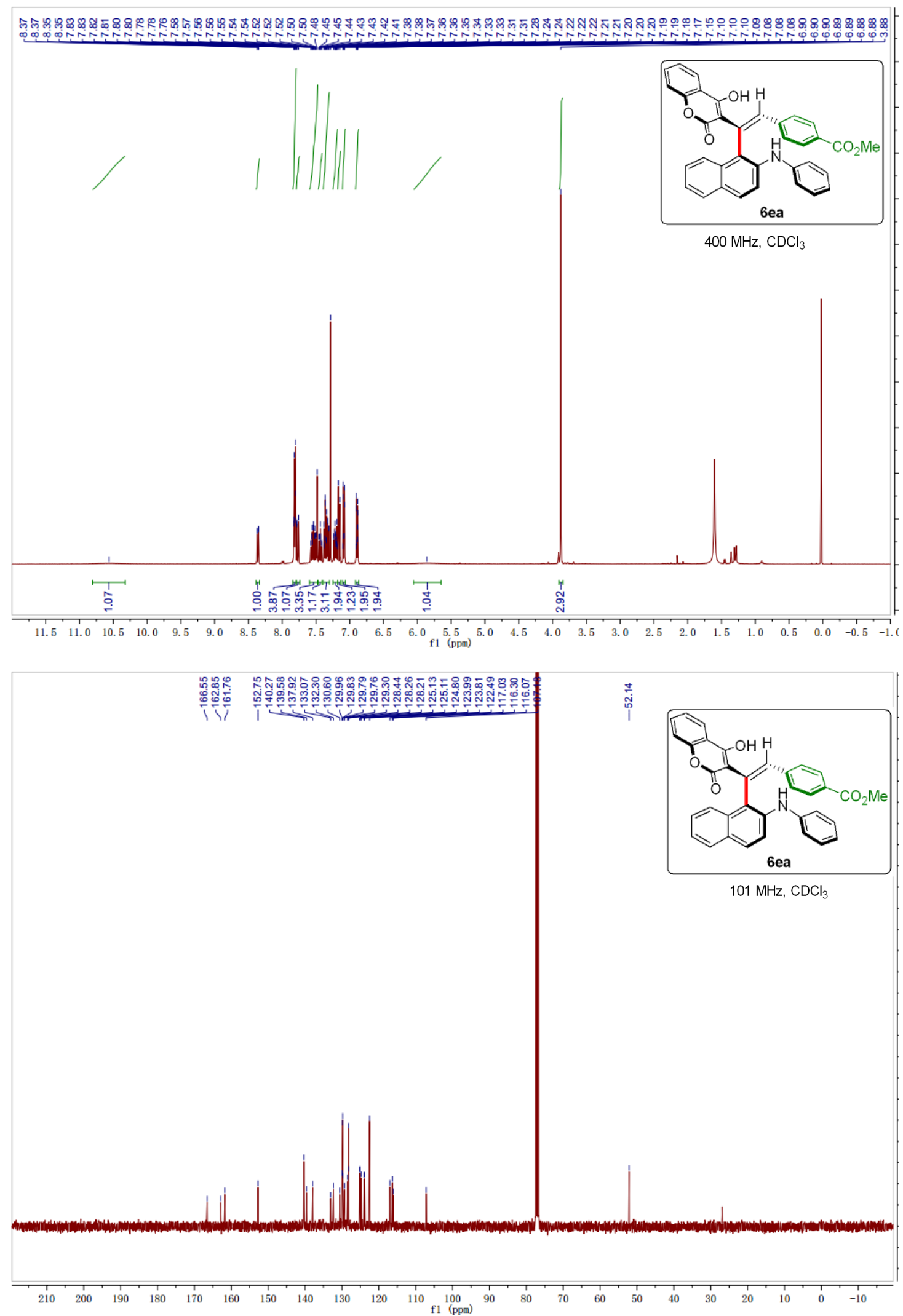

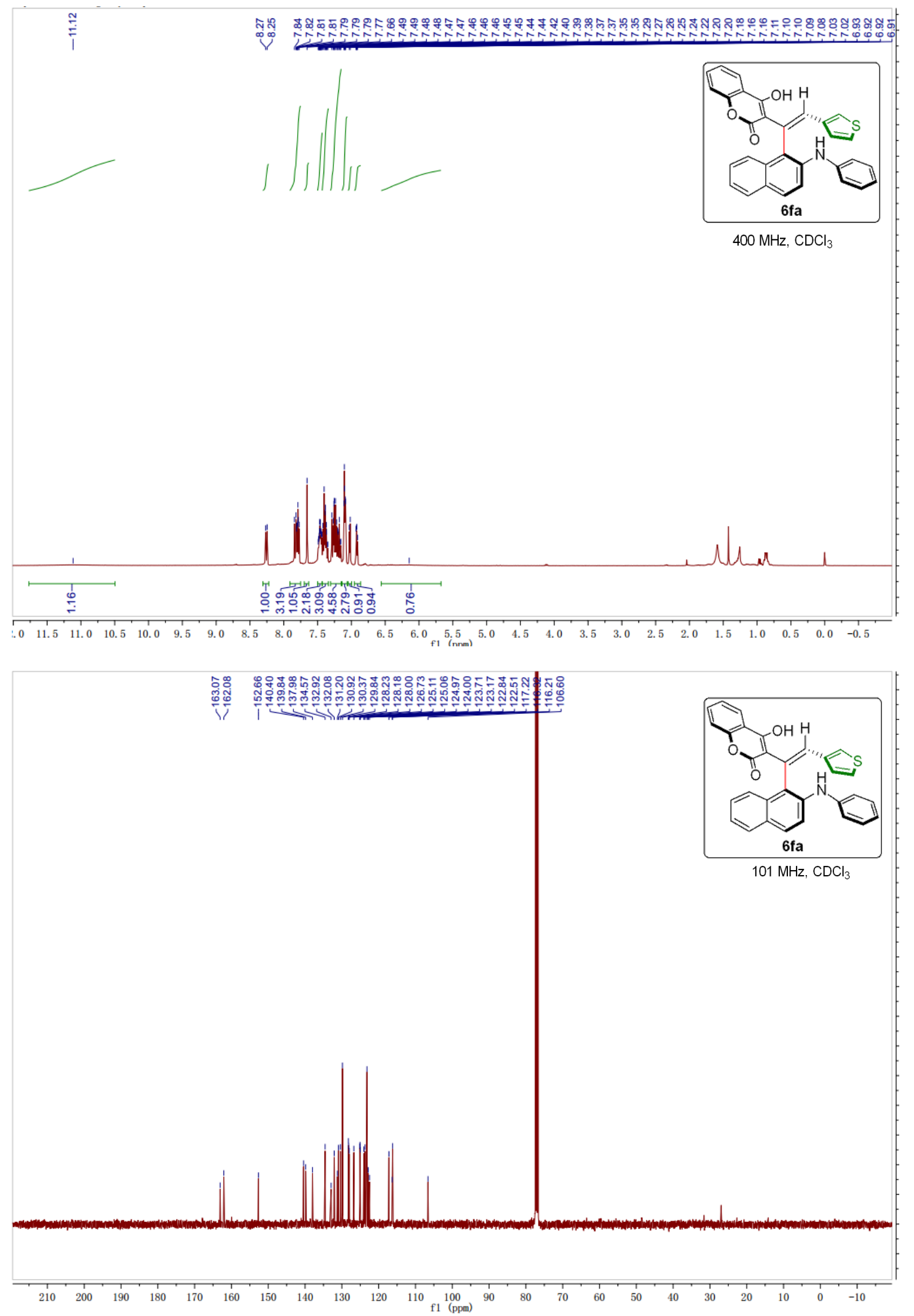

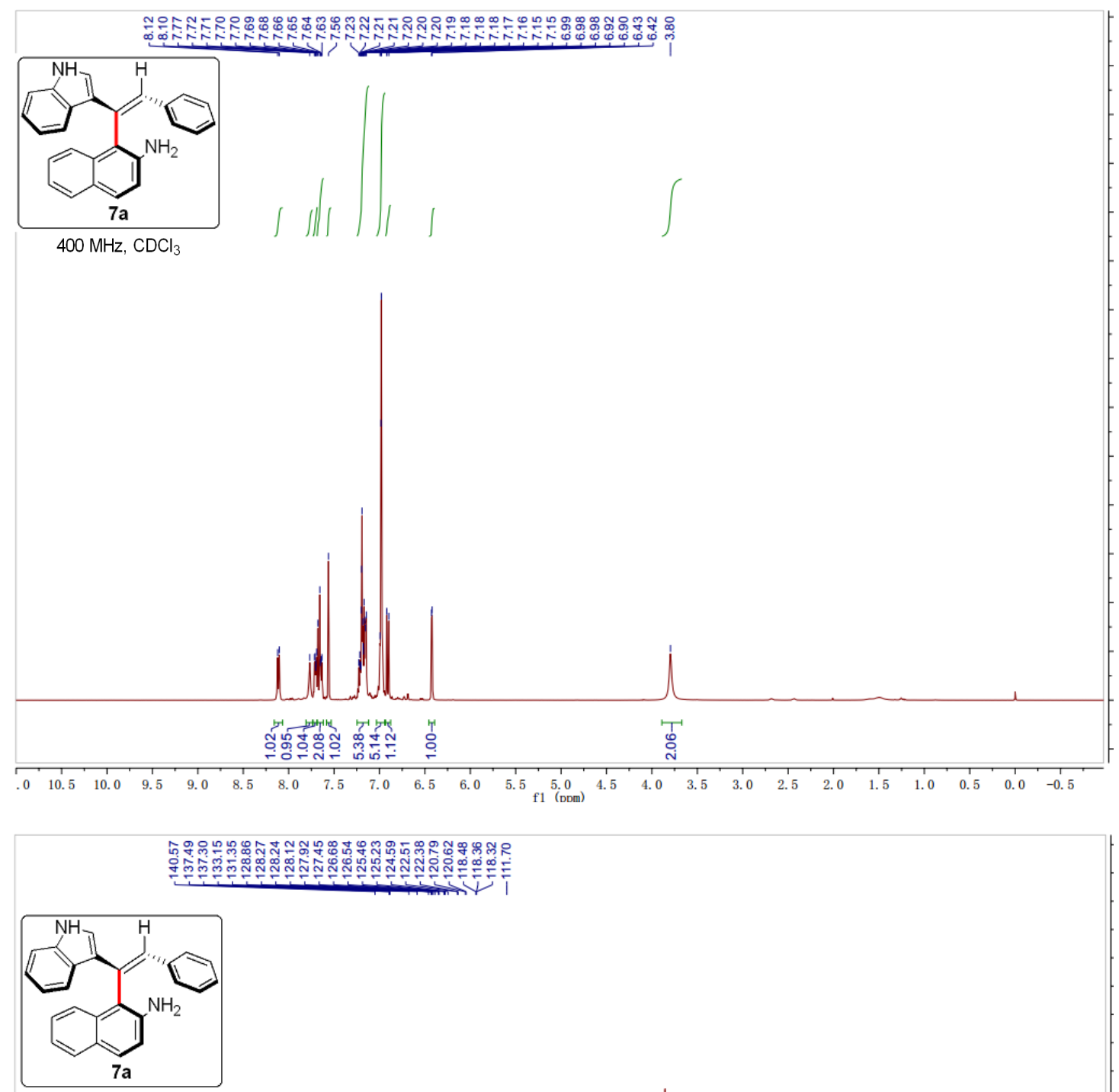

$101 \mathrm{MHz}, \mathrm{CDCl}_{3}$

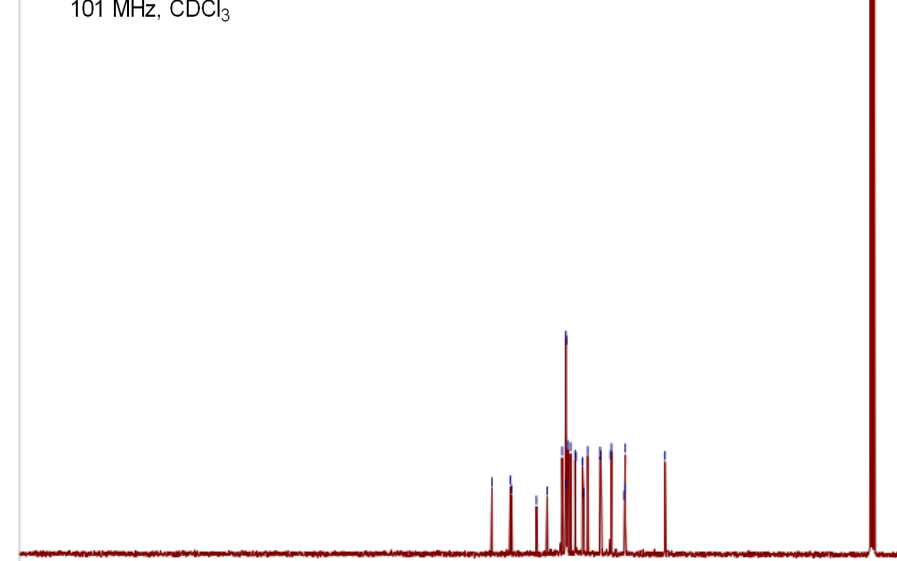

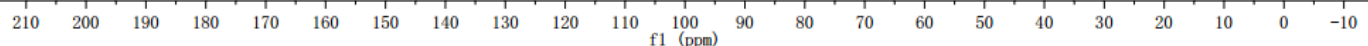




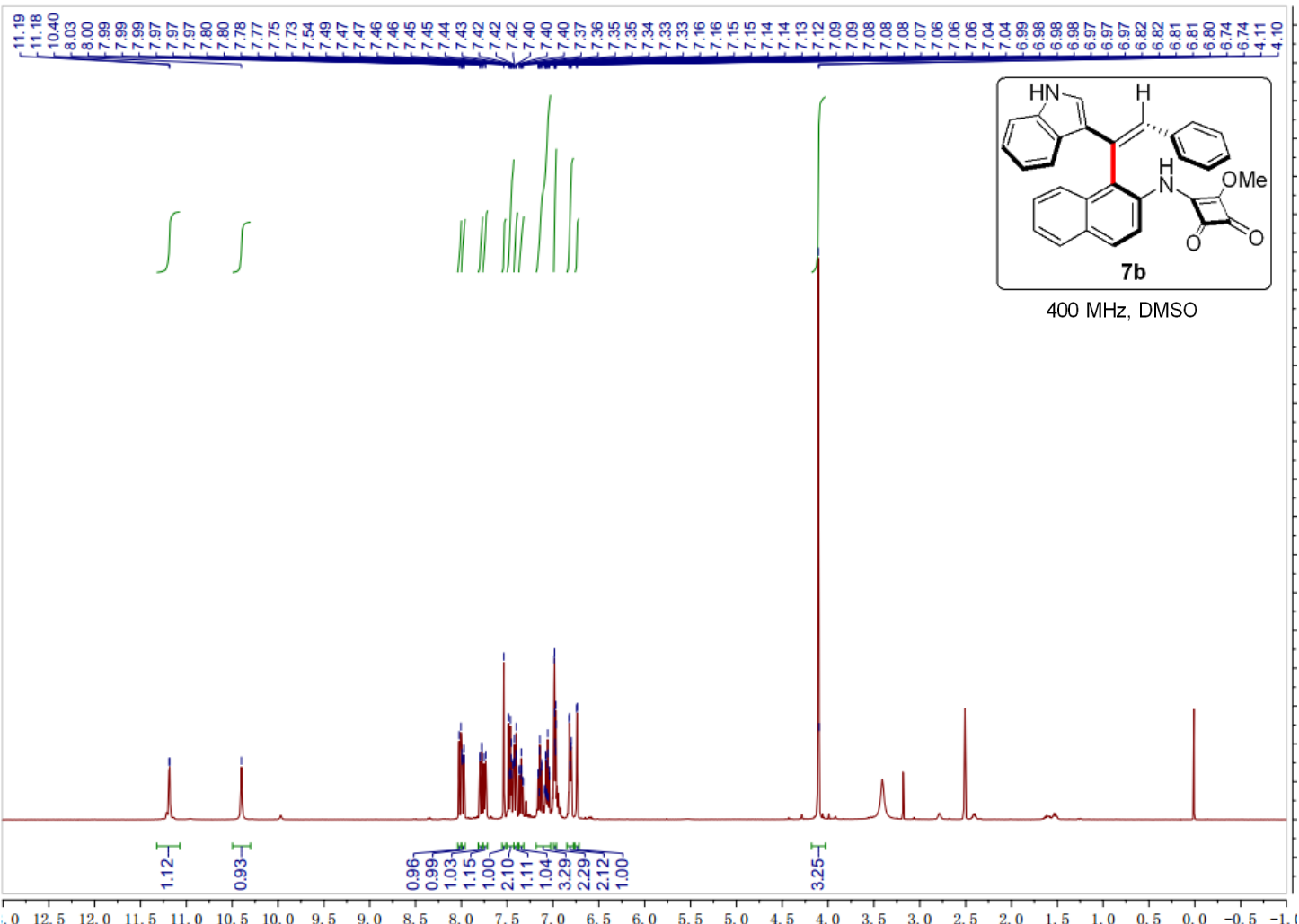

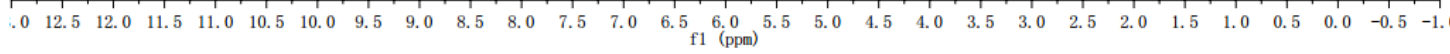

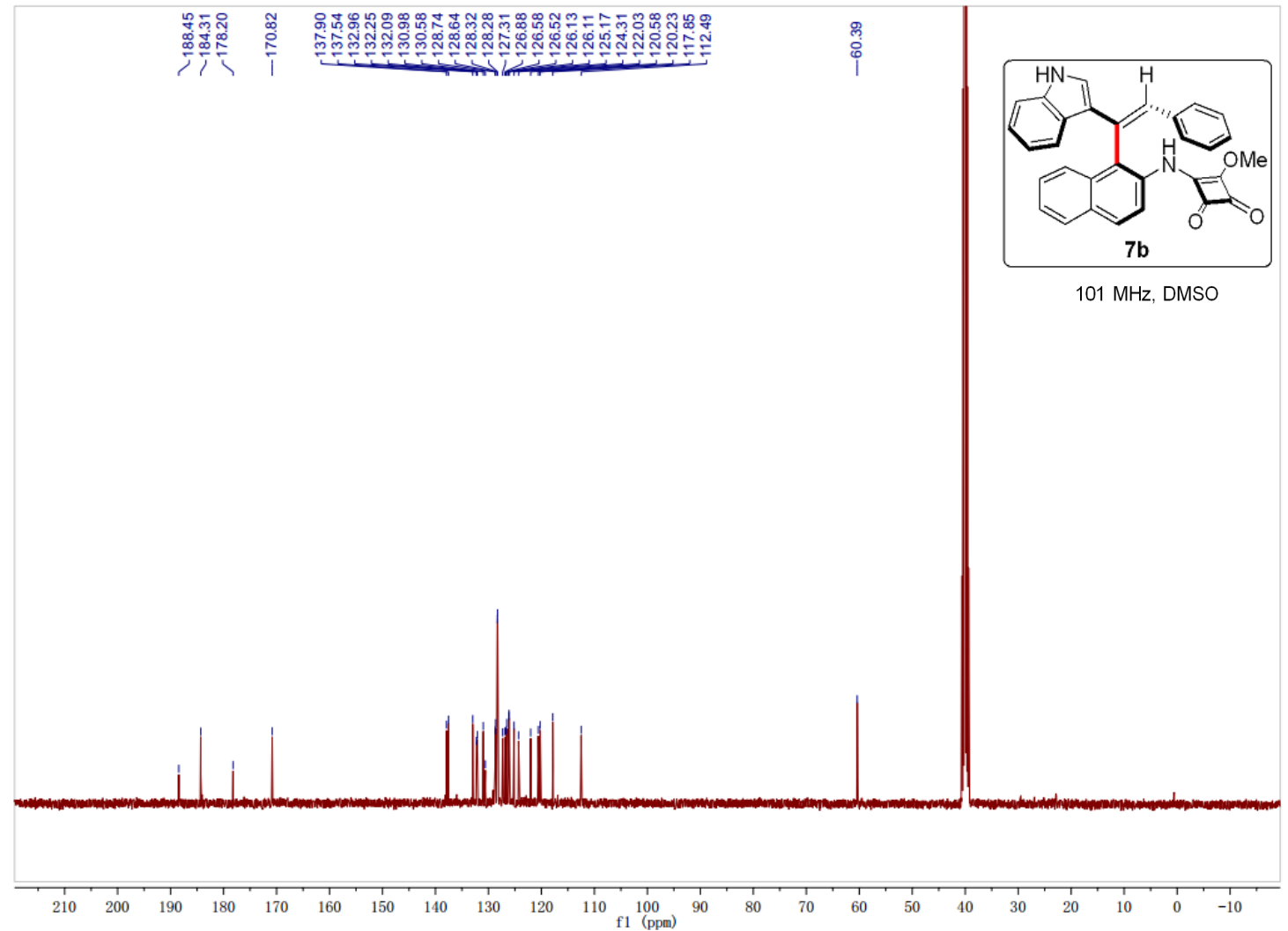



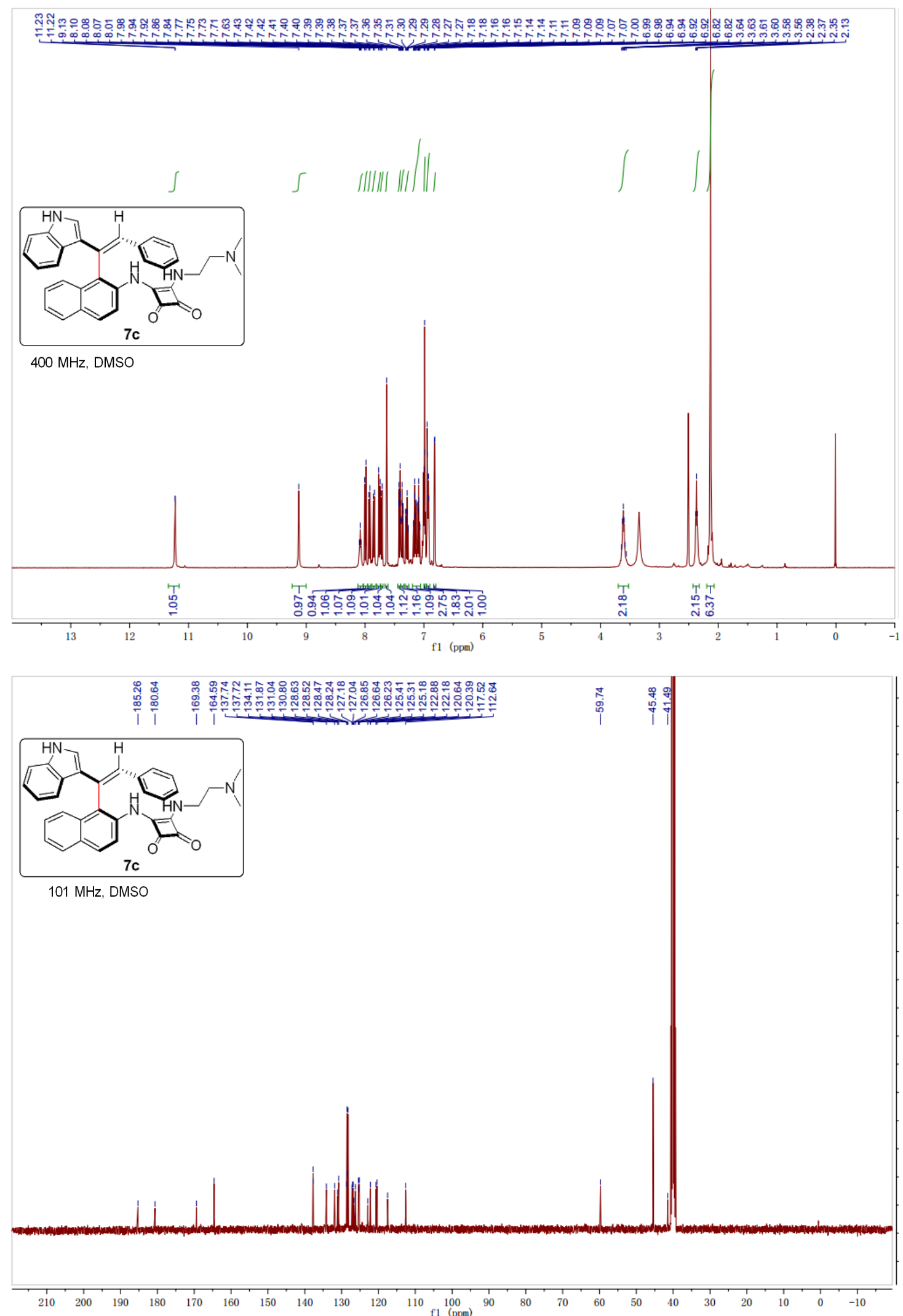